\title{
Information processing in the olfactory system of different amphibian species
}

\author{
Dissertation \\ for the award of the degree \\ "Doctor of Philosophy" \\ Division of Mathematics and Natural Sciences \\ of the Georg-August-Universität Göttingen
}

within the doctoral program International Max Planck Research School (IMPRS) for Neurosciences of the Georg-August University School of Science (GAUSS)

submitted by

Lukas Weiss

from Bozen

Göttingen 2020 


\section{Thesis Committee}

Prof. Dr. Ivan Manzini (Reviewer)

Department of Animal Physiology and Molecular Biomedicine, Institute of Animal Physiology, JustusLiebig-Universität, Gießen

Prof. Dr. Martin Göpfert (Reviewer)

Department of Cellular Neurobiology, Schwann-Schleiden Research Center, Georg-August-University, Göttingen

Dr. Camin Dean

Trans-synaptic Signaling Group, European Neuroscience Institute, Göttingen

\section{Members of the Examination Board}

Prof. Dr. Ivan Manzini (Reviewer)

Department of Animal Physiology and Molecular Biomedicine, Institute of Animal Physiology, JustusLiebig-Universität, Gießen

Prof. Dr. Martin Göpfert (Reviewer)

Department of Cellular Neurobiology, Schwann-Schleiden Research Center, Georg-August-University, Göttingen

\section{Further members of the Examination Board}

\section{Dr. Camin Dean}

Trans-synaptic Signaling Group, European Neuroscience Institute, Göttingen

Prof. Dr. Ralf Heinrich

Department of Cellular Neurobiology, Schwann-Schleiden Research Center, Georg-August-University, Göttingen

Prof. Dr. Tim Gollisch

Sensory Processing in the Retina, University Medical Center, Georg-August-University, Göttingen

Prof. Dr. Thomas Dresbach

Department of Anatomy and Embryology, Institute of Anatomy and Embryology, University Medical Center, Georg-August-University, Göttingen

Date of oral examination: 07.09.2020 
So long, and thanks for all the

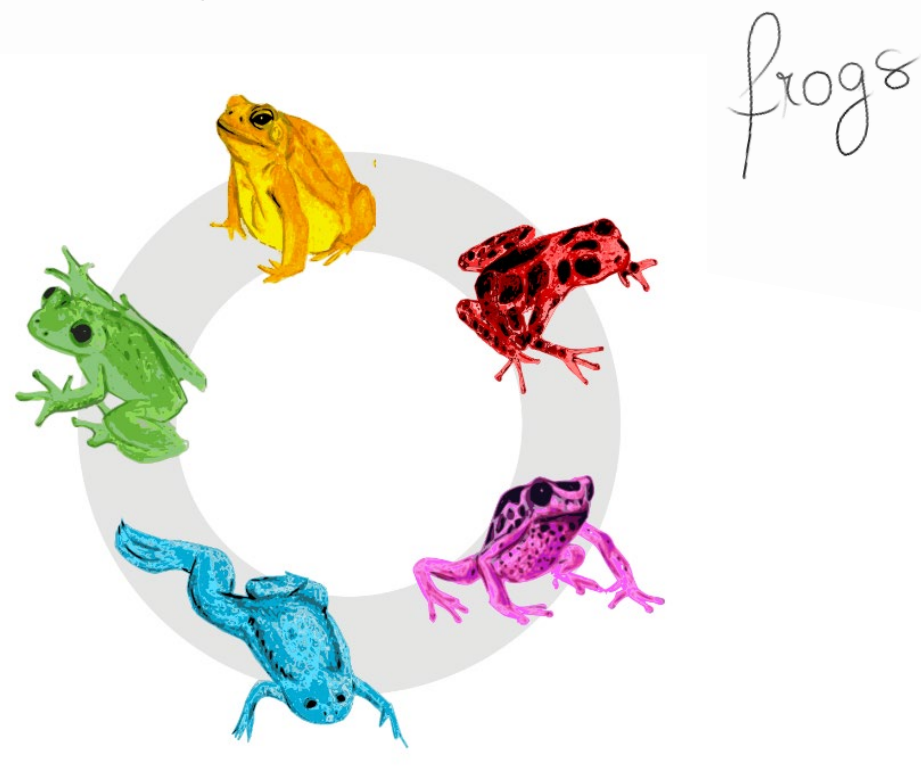

ii 


\section{Table of Contents}

CHAPTER 1: INTRODUCTION

CHEMORECEPTION AT THE EVOLUTIONARY TRANSITION BETWEen WATER AND LAND 1

PRINCIPLES OF OLFACTORY PROCESSING I: THE INVERTEBRATE SYSTEM

DIFFERENT MULTIGENE FAMILIES DETECT ODORANT MOLECULES

FROM THE SENSORY ORGANS TO THE BRAIN

PRINCIPLES OF OLFACTORY PROCESSING II: THE VERTEBRATES

OLFACTORY RECEPTORS AND THEIR LIGANDS

ODORANT RECEPTORS (ORS)

TRACE AMINE ASSOCIATED RECEPTORS

TYPE 1 VOMERONASAL RECEPTORS (V1RS)/ OLFACTORY RECEPTORS RELATED TO CLASS A GPCRS (ORA) 8

TYPE 2 VOMERONASAL RECEPTORS (V2RS)/ OLFC 9

OTHER OLFACTORY RECEPTOR FAMILIES

OLFACTORY RECEPTOR NEURONS: RECEPTOR EXPRESSION AND SIGNALING CASCADE

THE ONE NEURON - ONE RECEPTOR RULE

RN TYPES AND THEIR RECEPTORS $\quad 12$

INTRACELLULAR SIGNALING CASCADES

FUNCTIONAL AND MOLECULAR SEGREGATION OF OLFACTORY INPUT AND PROJECTIONS $\quad 14$

THE BIG DIVIDE: MAIN VS. ACCESSORY OLFACTORY SYSTEM $\quad 14$

FISHES: AN ALL IN ONE APPROACH TO OLFACTION

TETRAPODS: ANATOMICAL SEGREGATION OF SUBSYSTEMS $\quad 19$

EVOLUTIONARY DIFFERENCES IN THE OLFACTORY BULB CIRCUIT

OBJECTIVES AND STRUCTURE OF THIS THESIS

CHAPTER 2: DYE ELECTROPORATION AND IMAGING OF CALCIUM SIGNALING IN XENOPUS NERVOUS SYSTEM $\quad 28$

CHAPTER 3: CONSERVATION OF GLOMERULAR ORGANIZATION IN THE MAIN OLFACTORY \begin{tabular}{lr} 
BULB OF ANURAN LARVAE & 41 \\
\hline
\end{tabular}

CHAPTER 4: DEVELOPMENT OF NEURONAL CIRCUITS FOR AQUATIC AND AERIAL OLFACTION DURING AMPHIBIAN METAMORPHOSIS $\quad 52$

CHAPTER 5: MULTI-GLOMERULAR PROJECTION OF SINGLE OLFACTORY RECEPTOR NEURONS IS CONSERVED AMONG AMPHIBIANS $\quad 75$ 
THE RELATIVE IMPORTANCE OF OLFACTORY SUBSYSTEMS IN ANURANS 96

VARIABILITY VS. STABILITY IN THE LARVAL OLFACTORY SYSTEM 96

VARIABILITY VS. STABILITY IN THE METAMORPHOTIC AND POSTMETAMORPHOTIC OLFACTORY SYSTEM 100

THE ANURAN SYSTEM BETWEEN A RODENT MAIN AND ACCESSORY OLFACTORY SYSTEM? 106

MULTI-GLOMERULAR WIRING PATTERNS AS PARALLEL OLFACTORY CHANNELS ACROSS ANIMALS 107

\begin{tabular}{lr} 
SUMMARY & 110 \\
\hline
\end{tabular}

\begin{tabular}{lr} 
REFERENCES & 111 \\
\hline
\end{tabular}

\begin{tabular}{lr} 
ACKNOWLEDGEMENTS & 142 \\
\hline
\end{tabular}

\begin{tabular}{lr} 
LIST OF ABBREVIATIONS & 144 \\
\hline
\end{tabular}

\begin{tabular}{lr} 
LIST OF FIGURES & 144 \\
\hline
\end{tabular}

\begin{tabular}{lr} 
DECLARATION & 145 \\
\hline
\end{tabular}

CV: LUKAS WEISS $\quad 146$ 


\section{Chapter 1: Introduction}

\section{Chemoreception at the evolutionary transition between water and land}

The evolution of cellular life and the segregation of intra- and extracellular space came with the necessity of finding ways to detect chemicals on the outside and correctly perceive them as food, pathogens, or conspecifics. From food-driven chemotaxis in motile bacteria (Webre et al., 2003) to sophisticated odor-driven social behaviors in insects (Hansson and Stensmyr, 2011) or vertebrates (Bear et al., 2016), chemosensation is an impressive success story across the tree of life. To detect the myriad of different molecules in the outside world and assess their relevance, organisms had to develop receptors adapted to the specific chemo-space they are living in (Yohe and Brand, 2018). The remarkable adaptivity of the chemical senses particularly shows at a turning point in evolution from the dawn of the Silurian to the Carboniferous period (approx. $420-340$ million years ago), when millipedes, arachnids, hexapods and finally vertebrates independently conquered terrestrial habitats (Garwood and Edgecombe, 2011; Little, 1990; Ward et al., 2006). An enormous amount of whole genome sequencing has demonstrated, how the gene repertoire coding for chemical receptors, in particular odorant receptors, has changed at the transition from an aquatic to an aerial environment (Niimura, 2009; Vieira and Rozas, 2011). In the vertebrate lineage, the ancestors of the modern amphibians were the first ones to leave the water and their extant descendants still live a life that is partially reliant on the aquatic environment. Their olfactory system thus provides a formidable opportunity to dissect mechanisms from the molecular to the neuronal network level that proved to be adapted to both aquatic as well as aerial environments (Reiss and Eisthen, 2008).

\section{Principles of olfactory processing I: the invertebrate system}

There are several common molecular and anatomical features between the olfactory systems of different animal lineages (Bargmann, 2006). The first and arguably one of the most important steps in developing an efficient olfactory system is the expression of olfactory receptors in the membrane of specialized sensory cells, able to detect and distinguish molecules that are ecologically relevant for the organism.

\section{Different multigene families detect odorant molecules}

Several multigene families encoding for transmembrane proteins have been co-opted as chemosensors during animal evolution (Niimura and Nei, 2006; Robertson, 2015). In the following I will focus primarily on olfactory receptors, although they are highly related to other chemosensory receptors like gustatory receptors. Olfaction can be mediated by metabotropic as well as ionotropic receptor proteins. Most olfactory metabotropic receptors consist of seven transmembrane domains and are G protein coupled receptors (GPCRs) which transmit odorant-induced signal to intracellular G- 
proteins (Spehr and Munger, 2009). These G-proteins are enzymes that induce second messenger cascades which in turn depolarize the sensory cells. Chemosensory GPCRs are highly conserved among animals (Derby et al., 2016) and play a role e.g. in the mating cycle of yeast cells (Bardwell, 2005; Dohlman, 2002; Fredriksson et al., 2012), feeding related chemotaxis in the nematode Caenorhabditis elegans (Bargmann and Mori, 1997; Sengupta et al., 1996; Troemel et al., 1995), or mate-attraction in the marine mollusk Aplysia californica (Cummins et al., 2009). GPCRs function as chemosensors also in early diverging deuterostomes like starfish (Roberts et al., 2017) or the sea urchin (Raible et al., 2006), and constitute the vast majority of olfactory receptors in vertebrates (Niimura, 2009). The vertebrate olfactory system will be covered more extensively in the next part of this thesis.

The most species-rich lineage of animals are the arthropods, including chelicerata, myriapoda, crustacea and hexapoda (Eyun et al., 2017; Hansson and Stensmyr, 2011). Intriguingly, arthropods constitute the only major animal lineage that don't use GPCRs as chemosensors (Derby et al., 2016; Figure 1). One major group of olfactory receptors present in all arthropods are the ionotropic receptors (IRs) (Benton et al., 2009; Croset et al., 2010; Robertson, 2015), which have already emerged in basal protostomes (Croset et al., 2010). IR ligands described in arthropods are mostly amino acids, amines and ketones (Hallem and Carlson, 2006; Hallem et al., 2004; Mayer, 2005). As observed in most multigene families, the IR repertoire of different species varies quite substantially (Corey et al., 2013; Kozma et al., 2018; Terrapon et al., 2014; Vizueta et al., 2017; Yan et al., 2020).

The major family of olfactory receptors in insects are the odorant receptors (insect ORs; Figure 1) (Clyne et al., 1999; Gao and Chess, 1999; Vosshall et al., 1999). OR genes are an insect-specific expansion of a single lineage of the more ancient gene family of invertebrate gustatory receptors (GRs) (Robertson, 2019; Robertson et al., 2003). Both GRs and ORs share the seven transmembrane domains with the GPCRs but have an inverted membrane topology (Yan et al., 2020). Together with the broadly conserved odorant receptor co-receptor (Orco) (Gomez-Diaz et al., 2018; Larsson et al., 2004), ORs function as heteromeric cation-specific channels (Sato et al., 2008; Smart et al., 2008; Wicher et al., 2008). The insect-specific expansion of the OR genes is hypothesized to be linked to the terrestrialization of the insects from their aquatic ancestors. However, also crustaceans, myriapods and chelicerate arthropods have conquered land, but no OR genes are found in their genomes (Eyun et al., 2017; Robertson et al., 2003). Just like the IRs, the number of insect OR genes varies extensively, with e.g. only three OR genes found in the early diverging dragon fly (Brand et al., 2018) and more than 500 in the raider ant (McKenzie and Kronauer, 2018).

\section{From the sensory organs to the brain}

The adaptive capacities of the sense of smell particularly show in the evolutionary adoption of different and unrelated receptor gene families to match the demands of an ever-changing environment. The genetically encoded receptors are expressed on the cellular membrane of the dendrites of bipolar olfactory receptor neurons (RNs) (Hildebrand and Shepherd, 1997; Strausfeld and Hildebrand, 1999). Since animals originated in the water, these dendrites are encapsulated by a cuticular sensillum filled with an aqueous lymph in the arthropods (Breithaupt and Thiel, 2011; Zacharuk, 1980; Figure 1), or the equivalent epithelial mucosa of terrestrial vertebrates (Hildebrand and Shepherd, 1997). The 
olfactory organs of invertebrates are highly diverse in form and position on the body (Cummins et al., 2009; Hansson and Stensmyr, 2011; Hart and Chao, 2010; Harzsch and Krieger, 2018; Polese et al., 2016; Schneider, 1964; Van Wijk et al., 2006). A typical olfactory sensillum on the first antennal pair of the malacostracan crustaceans (aesthetascs) includes up to $400 \mathrm{RNs}$, with each aesthetasc putatively including an identical set of RNs (Harzsch and Krieger, 2018; Figure 1), while each insect sensilla generally includes one to four RNs (Zhao and McBride, 2020). In insects, the antennae is regarded as the major olfactory organ (Figure 1) and expresses both ORs and IRs in different types of sensilla (basiconic, trichoid, coeloconic, intermediate) (Benton et al., 2009; Fishilevich and Vosshall, 2005; Schneider, 1964; Zhao and McBride, 2020). The maxillary palps have only basiconic sensilla and their function is not totally clear and varies among species (Lu et al., 2007; Shiraiwa, 2008).

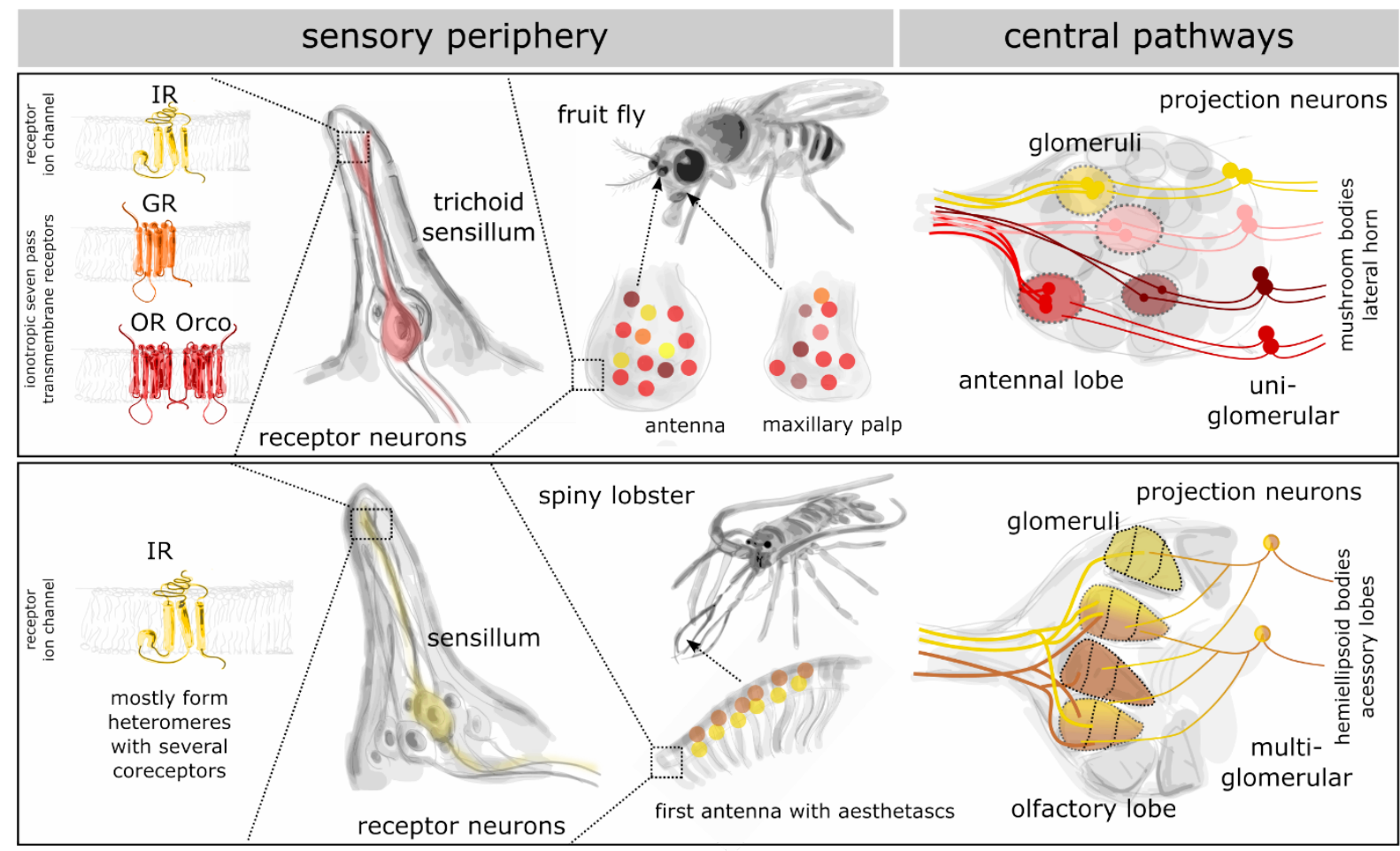

Figure 1 Olfactory pathways in invertebrates.

Olfactory pathways in invertebrates. The pathway from the olfactory periphery to the first relay station in the antennal lobe of the fruit fly (above) and the olfactory lobe of the spiny lobster (below) are depicted. Olfactory receptor proteins are expressed in the cell membranes of RNs. While insects generally use ORs, GRs and IRs, crustaceans are only reported to rely on IRs for olfaction. RNs are located in different types of sensilla on the sensory organs (antennae, palps). RN axons project to glomeruli in the antennal/olfactory lobe. While insect RNs are mostly unbranched, they show multi-glomerular projections in many crustaceans. Postsynaptic projection neurons convey the sensory information from the periphery to higher brain centers. Depicted data bases on the following reviews (Harzsch and Krieger, 2018; Yan et al., 2020; Zhao and McBride, 2020). IR ionotropic receptor, GR gustatory receptor, OR odorant receptor, Orco odorant receptor coreceptor. 
Hydrophobic chemicals enter the sensilla of insects through pores in the cuticle and bind to the olfactory binding protein (Vieira and Rozas, 2011; Vogt and Riddiford, 1981), through which they are transported to the receptors. Individual RNs in insects express either a single functional OR, which appears to be a hetero-tetrameric ion channel consisting of two copies of Orco plus two ligand-specific OR copies (Fishilevich and Vosshall, 2005; Sato et al., 2008; Yan et al., 2020) or a single IR-heteromere consisting of one ligand-specific allele of the IR family plus one of several common IR-coreceptors (Abuin et al., 2012; Benton et al., 2009). However, this rule is by no means absolute, with several instances of receptor co-expression reported (Benton et al., 2009; Fishilevich and Vosshall, 2005; Jones et al., 2007). The single ligand-specific receptor allele expressed in each RN is a feature that most probably has evolved several times in the animals kingdom, since it is a fundamental principle of olfaction also in vertebrates (Chess et al., 1994; Serizawa et al., 2006). Contrastingly, the 32 RNs in C. elegans each are thought to express several of the approx. 1000 chemoreceptors (Bargmann, 2006; McCarroll et al., 2005; Troemel et al., 1995) and are responsive to a variety of different, putatively also structurally unrelated chemicals.

The olfactory receptors in the dendrites function on a continuous spectrum from generalist to absolute specialist (Hallem and Carlson, 2006). While generalist receptors bind and detect a broad range of different but structurally similar odor molecules, the absolute specialists function as detectors for a single odorant cue that mostly fulfills a specific ecological role (Hallem and Carlson, 2006; Hallem et al., 2004). Many such specialists or narrowly tuned receptors have been identified in various species, e.g. the geosmin receptor in Drosophila (Stensmyr et al., 2012) or mosquito receptors narrowly tuned to compounds in human sweat (Carey et al., 2010). In contrast, e.g. the receptors detecting fruit odors in the mosquito are very broadly tuned and unspecific, inversely to the ones in the fruit flies (Carey et al., 2010; Hallem et al., 2004). However, tuning specificity of odorant receptors also highly depends on odorant concentration (Andersson et al., 2012).

In insects, RNs expressing the same ligand-specific OR allele generally project their axon towards a single ovoid neuropil structure in the antennal lobe, called a glomerulus (Couto et al., 2005; Fishilevich and Vosshall, 2005; Hansson and Stensmyr, 2011; Hansson et al., 1992; Riabinina et al., 2016; Zhao and McBride, 2020; Figure 1). This pattern is generally assumed to apply for both IRs and ORs (Benton et al., 2009). Each glomerulus thus receives the odorant information detected by a single receptor. Odorant molecules detected by a variety of receptors induce neuronal activity in multiple glomeruli, while odorants detected by a single specialized receptor only activate a single glomerulus (Couto et al., 2005; Fishilevich and Vosshall, 2005; Hansson and Stensmyr, 2011). In Drosophila, RNs encapsulated in different sensilla project to different locations of the antennal lobe (Couto et al., 2005), putatively constituting functionally distinct information channels (Hallem and Carlson, 2006; Yan et al., 2020). The input organization of olfactory input in glomerular structures has most probably evolved independently multiple time, since it has been found in various animal lineages (Beltz et al., 2003; Brownwell, 1998; Chase and Tolloczko, 1986; Harzsch and Krieger, 2018; Sombke et al., 2012; Strausfeld and Hildebrand, 1999) but is absent in others (Bargmann, 2006; Bargmann and Mori, 1997). Several deviations of the one receptor-one population of RNs-one glomerulus-rule have been described in invertebrates (Ernst et al., 1983; Hansson and Stensmyr, 2011; Harzsch and Krieger, 2018; 
Ignell et al., 2001; Schmidt et al., 1992; Figure 1). Their respective function remains unknown up to date.

Within the glomeruli, synapses to second order projection neurons are formed (Figure 1). These projection neurons extend their dendrites towards the glomeruli, where they branch extensively (Zhao and McBride, 2020). In most insects, the projection neurons have been described as forming connections to one sole glomerulus, thus receiving information from a single odorant receptor (Figure 1). However, multiple sources describe the occurrence of insect projection neurons connecting to multiple glomeruli (Hansson and Stensmyr, 2011; Homberg et al., 1988; Ignell et al., 2001; Stocker et al., 1990). In the lobster, all projection neurons have been found to be multi-glomerular, some cells even innervating up to $80 \%$ of all glomeruli in the olfactory lobe (Wachowiak and Ache, 1994; Figure 1). Whether these special cases serve a distinct function remains elusive.

The organization of the olfactory system in invertebrates has many similarities to the vertebrate system. Particularly striking are the parallels between insects and vertebrates, constituting a prime example of convergent evolution driven by environmental demands.

\section{Principles of olfactory processing II: the vertebrates}

\section{Olfactory receptors and their ligands}

As in invertebrates, the detection of odorant molecules relies on the presence of receptor proteins in the membranes of RNs in dedicated peripheral olfactory organs (Bear et al., 2016; Figure 2A). Multigene families encoding for these proteins are found in all vertebrate genomes (Figure 2B), nearly all of them are seven transmembrane GPCRs (Bear et al., 2016; Manzini and Korsching, 2011). Even though they all have first been described in rodents, I will introduce the various gene families in the approximate evolutionary order, in which they were recruited as olfactory receptors.

\section{Odorant receptors (ORs)}

The first rhodopsin-type GPCR multigene family was named 'odorant receptors' (ORs) at their discovery in the rat genome (Buck and Axel, 1991) and has been shown to even predate the emergence of the first vertebrates (Churcher and Taylor, 2009, 2010; Niimura, 2009; Figure 2). Over the last 30 years, ORs have been identified as olfactory receptors in all major vertebrate lineages: agnathans (Freitag et al., 1999; Libants et al., 2009), fishes (Barth et al., 1997; Cao et al., 1998; Dugas and Ngai, 2001; Kondo et al., 2002; Ngai et al., 2003; Sun et al., 1999), amphibians (Freitag et al., 1998; Mezler et al., 1999), birds (Nef et al., 1996) and mammals (Buck and Axel, 1991; Niimura and Nei, 2007; Zhang and Firestein, 2002).

The vertebrate ORs are not related to the insect ORs, but show similar evolutionary dynamics of gene loss and expansion resulting in very different numbers of functional OR genes in ecologically diverse species (Nei and Rooney, 2005; Niimura, 2009; Figure 2B). The vertebrate OR genome is generally subdivided into Type 1 and Type 2 genes, and each of them again into several subfamilies (Niimura, 
2009). During the transition to life on land, two subfamilies of Type 1 ORs ( $\alpha$ and $\gamma$ ) presumably proved more useful to detect volatile odorants, which led to an enormous expansion of these subfamilies in terrestrial vertebrates, the tetrapods (Niimura, 2009, Figure 2B). These genes have on the other hand mostly been lost in the teleost fishes (Niimura, 2009; Figur 2B). A different nomenclature is usually used for mammalian ORs, which have been subdivided into class I ORs (subfamily $\alpha$ and $\beta$ ) and class II ORs ( $\gamma$ ) (Glusman et al., 2000; Niimura and Nei, 2006).

In mammals, ligands for about 100 individual ORs have been identified (Krautwurst et al., 1998; Malnic, 2007; Malnic et al., 1999; Nara et al., 2011; Saito et al., 2009; Zhao et al., 1998). They mostly detect small volatile molecules like aldehydes, esters, alcohols, ketones etc. in a combinatorial way. Each OR recognizes multiple odorant molecules and each odor can bind to multiple ORs. In this way, millions of different odors can be distinguished (Malnic, 2007; Malnic et al., 1999; Nara et al., 2011). Like in insects, tuning of individual mammalian ORs can be broad or rather narrow, making some receptors generalist and others specialists (Grosmaitre et al., 2009; Kepchia et al., 2017; Nara et al., 2011). In an extensive study on the ligand specificity of OR genes, Saito and colleagues have identified that mammalian class I ORs are generally detecting more hydrophilic compounds than class II ORs (Saito et al., 2009). This would also explain, why especially the class II OR repertoire has expanded drastically in most terrestrial mammal genomes (Niimura, 2009; Figure 2B).

An enormous expansion of the $\mathrm{OR}$ genome has taken place at the transition from aquatic to terrestrial life. The OR family was possibly better suited to adapt to the volatile compound detection necessary to survive on land than members of other olfactory receptor families (Niimura, 2009). This in turn suggests that earlier diverging vertebrates rely on other olfactory receptor types (Figure 2B).

\section{Trace amine associated receptors}

A second multi-gene family coding for olfactory receptors are the trace amine associated receptors (TAARs; Figure 2). (Liberles and Buck, 2006; Lindemann and Hoener, 2005; Xu and Li, 2020). Like the ORs, TAARs are also rhodopsin-like GPCRs and are distantly related to receptors detecting biogenic amines like serotonin or dopamine (Borowsky et al., 2001; Bunzow et al., 2001). TAAR-like genes have been identified in the genome of the sea lamprey Petromyzon marinus (Hashiguchi and Nishida, 2007; Scott et al., 2019; Smith et al., 2013; Figure 2B), but their placement in the TAAR family is still disputed (Hussain et al., 2009; Xu and Li, 2020). Several members of the TAAR gene family have been identified in cartilaginous fishes (Marra et al., 2019; Sharma et al., 2019; Figure 2B) and a particularly big repertoire of TAARs in ray-finned fishes (Azzouzi et al., 2015; Gloriam et al., 2005; Hashiguchi and Nishida, 2007; Hussain et al., 2009). The enormous variation of the TAAR repertoire of teleost fishes suggests a crucial and diverse function of TAARs in aquatic olfaction (Azzouzi et al., 2015; Hashiguchi and Nishida, 2007; Hussain et al., 2009; Jiang et al., 2019; Xu and Li, 2020). In comparison, fewer TAAR genes are present in the genomes of later diverging vertebrates ( 6 in humans, 6 in The Western Clawed frog, 15 in the mouse, 17 in the rat compared to 100 in zebrafish) (Gloriam et al., 2005; Hashiguchi and Nishida, 2007; Hussain et al., 2013; Liberles and Buck, 2006; Lindemann et al., 2005). The TAAR genes in primates show an accelerated degeneration also in comparison to other mammals, putatively linked to the development of arboreal lifestyles (Eyun, 2019; Figure 2B). 
A

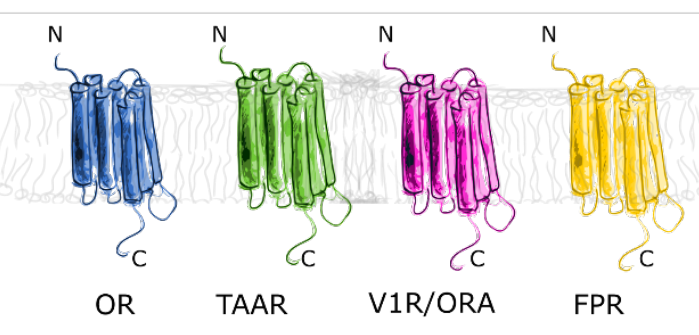

type A 'Rhodopsin-like'

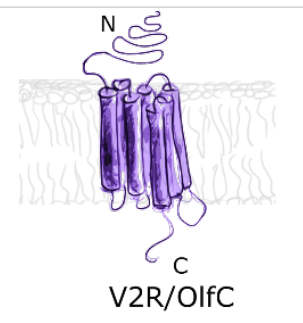

type C -metabotropic Glutamate receptor-like

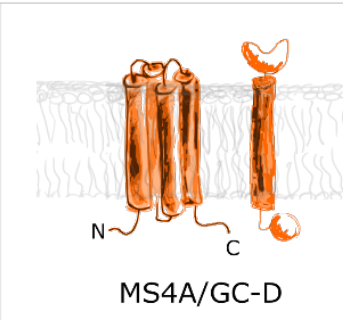

four pass transmembrane receptor/

Receptor guanyly cyclase

seven pass transmembrane G-protein coupled receptor

B

$$
\text { phylogeny }
$$

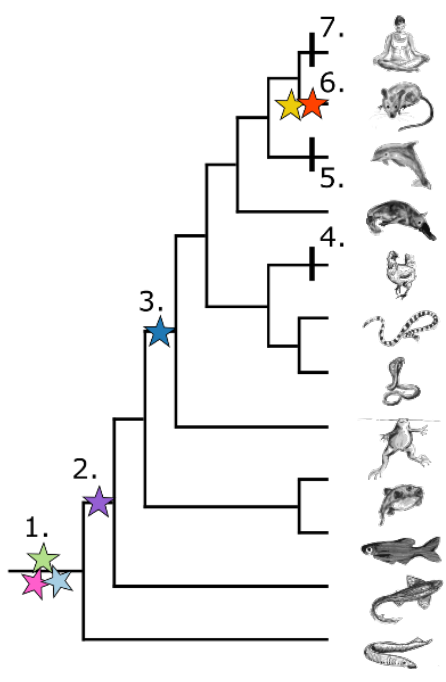

habitat

receptor repertoire

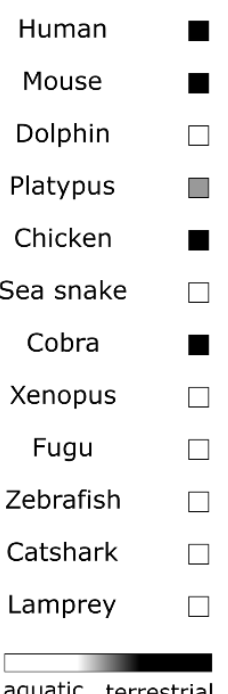

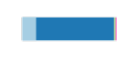

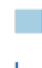

I
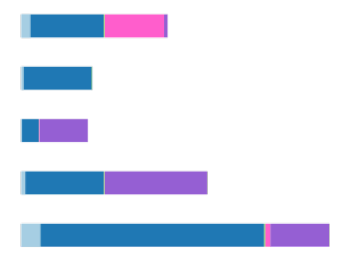

I

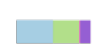

I

$\square$
0

$\%$ share

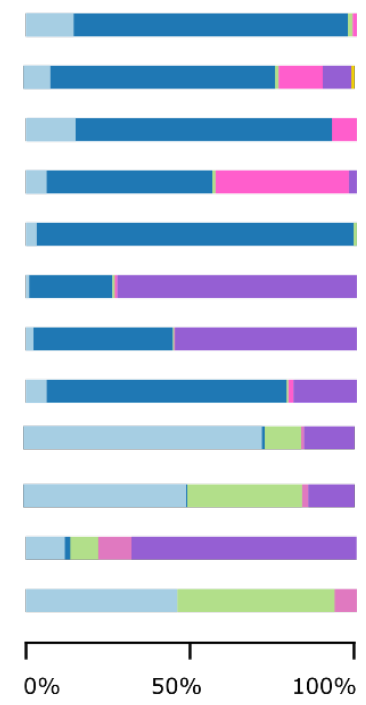

\section{Figure 2 Olfactory receptors in vertebrates.}

A) Vertebrate olfactory receptor proteins are mostly GPCRs with seven transmembrane domains belonging to either type $A$ or type $C$, with the exception of the membrane-spanning 4A proteins (MS4A), which often colocalize with the Guanylyl cyclase D (GC-D). B) A simplified phylogenetic overview of the different olfactory receptor gene families and their distribution in different vertebrate lineages is shown. Habitat, total number of olfactory gene families and the percentual share of the gene families relative to the entire olfactory gene repertoires are depicted. The numbers and stars in the phylogenetic tree highlight the estimated evolutionary timepoint, at which the recruitment of different receptor families occurred. The black bars indicate major loss of olfactory genes or entire gene families. 1. The olfactory receptor gene repertoire of the earliest vertebrates consisted of ORs (blue star), V1Rs (pink) and TAAR-like receptor genes (green). 2. V2Rs/OlfC genes (purple) were recruited after the divergence of the jawed vertebrates. 3. An enormous expansion of one subfamily of OR genes (dark blue) occurred with the evolution of the first terrestrial vertebrates (tetrapods). 4. A drastic loss of vomeronasal receptors of type 1 and 2 occurred in the bird lineage. 5 . Whales and dolphins have a very reduced olfactory gene repertoire and have almost lost their capability to smell. 6. FPR- (yellow) and MS4A- (orange) related olfaction has so far only been demonstrated in rodents. 7. The vomeronasal receptor repertoire in primates is significantly 
reduced. The numbers of genes represented in the bar plots is based on the following publications (Kishida et al., 2015, 2019; Libants et al., 2009; Liberles et al., 2009; Nei et al., 2008; Saraiva and Korsching, 2007; Sharma et al., 2019). FPR formyl peptide receptor, GC-D receptor guanyly cyclase type $D$, GPCR G protein coupled receptors, MS4A membrane-spanning 4A receptor, OR OR-type olfactory receptor, OR II mammalian class II/Y subfamily of ORs, TAAR trace amine associated receptor, V1R vomeronasal type 1 receptor, V2R vomeronasal type 2 receptor

The ligands for TAAR receptors are chemically similar to biogenic amines, but their endogenous concentration is much lower, hence their name trace amines (Berry, 2004; Xu and Li, 2020). Volatile trace amines are often formed by decarboxylation of amino acids. They are present in animal body fluids or decaying organic matter, linking them to innate ethological odor-guided circuits (Xu and Li, 2020). TAARs are thus involved e.g. in the attraction of female sea lampreys by a component of male semen (Scott et al., 2019), predator-cue detection in mice (Ferrero et al., 2011) or aversive behavior triggered by the death-associated odor cadaverine in zebrafish (Hussain et al., 2013). Due to their involvement in sexual behavior in many species, some TAARs can be considered examples for pheromone detectors. Pheromones are per definition "substances which are secreted to the outside by an individual and received by a second individual of the same species, in which they release a specific reaction, for example, a definite behavior or a developmental process" (Karlson and Lüscher, 1959). Originally, the pheromone system in vertebrates was believed to be primarily conveyed via different receptors, the vomeronasal receptors.

\section{Type 1 vomeronasal receptors (V1Rs)/ olfactory receptors related to class A GPCRs (ORA)}

Type 1 vomeronasal receptors (V1Rs) were initially found to be expressed in the sensory epithelium of the vomeronasal organ (VNO), an anatomically distinct olfactory organ present in most terrestrial tetrapods, but with an unclear origin and exact function (Baxi et al., 2006; Dulac and Axel, 1995; Eisthen, 1997). However, the involvement of V1Rs genes in olfaction predates the vomeronasal organ, which led to the renaming of the receptor family in fishes to olfactory receptor type A GPCRs (ORA) (Pfister and Rodriguez, 2005; Saraiva and Korsching, 2007; Figure 2A). Functional ORA genes were found in the sea lamprey (Grus and Zhang, 2009; Libants et al., 2009), the elephant shark (Grus and Zhang, 2009; Venkatesh et al., 2014) and six highly conserved genes in most teleost fish species (Johnstone et al., 2012; Nikaido, 2019; Ota et al., 2012; Saraiva and Korsching, 2007; Shi and Zhang, 2007; Zhu et al., 2016; Figure 2B). The remarkable constancy of the ORA genome in teleosts might stem from a highly conserved function, putatively involved in mating behavior (Johansson and Banks, 2010, 2011).

Contrastingly, the tetrapod ORA/V1Rs have started to radiate and diversify after the divergence from the fishes, forming the tetrapod-like lineages of the V1Rs (Saraiva and Korsching, 2007; Shi and Zhang, 2007; Figure 2B). The tetrapod V1R genes have about 20 members in the coelacanth, the closest extant relative of the tetrapods (Nikaido et al., 2013a), a similar number in the Clawed frog (Date-Ito et al., 2008; Saraiva and Korsching, 2007; Shi and Zhang, 2007), but almost no members in lizards, turtles, snakes or birds (Silva and Antunes, 2017; Figure 2B). In mammals, the numbers vary from 270 intact genes in the platypus (Grus et al., 2007; Nikaido, 2019; Rodriguez et al., 2002; Warren et al., 2008) to 
a very reduced repertoire in primates (Yoder and Larsen, 2014) and the complete absence of V1Rs in dolphins and some bat species (Young et al., 2010).

Since their discovery in the VNO of rats, the V1Rs have been proposed to detect volatile pheromones important for social and sexual communication (Boschat et al., 2002; Dulac and Axel, 1995). Mice lacking major parts of their V1R coding region were found to display altered sexual and aggression related behavior (Del Punta et al., 2002a). In an attempt to unravel the vomeronasal function, Isogai and colleagues identified several V1Rs responsible to specifically bind sulfated steroids both from conspecifics and predators (Isogai et al., 2011). The involvement of steroid derived compounds have already been identified as mediating several social and sexual behaviors in lampreys (Sorensen et al., 2005), frogs (Sansone et al., 2015) and different species of fishes (Cole and Stacey, 2006; Murphy et al., 2001). Additionally, teleost ORAs are involved in the detection of bile acids as attractive pheromones, (Buchinger et al., 2014; Cong et al., 2019) and oviposition cues in zebrafish (Ahuja and Korsching, 2014; Behrens et al., 2014).

\section{Type 2 vomeronasal receptors (V2Rs)/ OlfC}

A second vomeronasal receptor family was discovered in a different subset of neurons in the VNO of rodents shortly after the discovery of the V1Rs, and thus named vomeronasal type 2 receptors (V2Rs) (Herrada and Dulac, 1997; Matsunami and Buck, 1997; Ryba et al., 1997; Figure 2A). In contrast to the already presented vertebrate olfactory receptor families, the V2Rs belong to class $C$ of GPCRs. Class $C$ GPCRs are closely related to the metabotropic glutamate receptors and structurally distinct by a larger extracellular N-terminus (Manzini and Korsching, 2011). Their recruitment as olfactory receptors most probably happened after the divergence of the jawless fishes (purple star in phylogenetic tree, Figure $2 B$ ), since no related olfactory genes could be identified in the olfactory organ of lampreys (Libants et al., 2009).

Since fishes lack a VNO, the family coding for the V2R-related genes is described with the name olfactory class C GPCRs (OlfC) (Alioto and Ngai, 2006; Korsching, 2008). In cartilaginous fishes, the $\mathrm{V} 2 \mathrm{R} / \mathrm{OlfC}$ genes seem to be the major player for olfaction and make up almost their entire olfactory genome (Sharma et al., 2019; Venkatesh et al., 2014; Figure 2B). In teleost fishes, the number of OlfC family members varies extensively, in contrast to the ORA genes (Bjarnadóttir et al., 2005; Cao et al., 1998; Johnstone et al., 2009; Naito et al., 1998; Nikaido et al., 2013b; Yang et al., 2019). A series of molecular, physiological and behavioral data strongly suggests that OlfCs are involved in sensing of amino acids, a putative food odor in fishes (Alioto and Ngai, 2006; Luu et al., 2004; Sorensen and Caprio, 1998; Speca et al., 1999). Expansions of the OlfC repertoire in specific fish lineages are thus hypothesized to reflect the diversity of trophic stimuli (possibly amino acids or derivates) in their specific ecosystem (Nikaido et al., 2013b). Similarly, the high diversity of genes belonging to this family in the sharks could be feeding related (Sharma et al., 2019).

The biggest V2R repertoire identified up to date is found in the genome of the secondarily aquatic Western Clawed frog with 270 intact and more than 400 nonfunctional genes (Hagino-Yamagishi et al., 2004; Nei et al., 2008; Shi and Zhang, 2007; Silva and Antunes, 2017). Apparently, a huge lineage- 
specific expansion has taken place in the amphibians (Shi and Zhang, 2007; Figure 2B). As a caveat, it is to mention that much fewer V2Rs have been identified in the red legged salamander and not many different genomes are available to fully understand this expansion (Kiemnec-Tyburczy et al., 2012; Silva and Antunes, 2017). It thus remains elusive, which ligands are detected by the amphibian V2Rs, but some evidence suggests that they might also be detecting amino acids (Syed et al., 2013, 2017).

In later diverging vertebrates, the V2R genome is also quite variable (Shi and Zhang, 2007). While reptiles mostly have quite a big V2R repertoire (Figure $2 \mathrm{~B}$ ), especially when compared to the almost absent V1Rs (Brykczynska et al., 2013; Kishida et al., 2019; Silva and Antunes, 2017), several lineages of mammals completely lack functional V2Rs (Shi and Zhang, 2007; Young and Trask, 2007) and no V2R genes were found in the chicken genome. Evidence mostly from rodents models suggests that the V2Rs are involved in the detection of water-soluble pheromones (Brennan and Zufall, 2006; Haga et al., 2010; Kimoto et al., 2005; Leinders-Zufall et al., 2004). In an impressively complete study, a peptide secreted by the lacrimal gland of the male mouse enhanced female sexual behavior via the V2Rp5 receptor (Haga et al., 2010).

It is hypothesized, that during the evolutionary transition from water to land, the vertebrate repertoire of the V1Rs would have expanded to adapt to volatile pheromonal communications vs. waterborne pheromones detected by V2Rs (Shi and Zhang, 2007). However, while this theory finds support in the absence of functional V2Rs in most terrestrial mammals, reptiles have almost completely lost their V1Rs while maintaining a quite substantial V2R repertoire (Silva and Antunes, 2017).

\section{Other olfactory receptor families}

Apart from the four bigger gene families established as olfactory receptors in vertebrates, there are some other receptor types recently discovered to have olfactory functions. Some members of the Formyl peptide receptors (FPRs) have been found to be expressed in RNs in the VNO of rodents (Liberles et al., 2009; Rivière et al., 2009). These receptors have apparently been co-opted into the olfactory gene canon from the immune system (Migeotte et al., 2006), hence they are involved in the detection of pathogens or diseased conspecifics (Liberles et al., 2009; Rivière et al., 2009). Since rodents have been exceptionally able to recognize parasitized and sick conspecifics, this particular subset of olfactory receptors might also be rodent-specific (Kavaliers et al., 2005; Liberles et al., 2009; yellow star in the tree in Figure 2B). The latest addition to the canon of known olfactory receptors are the membrane spanning 4-pass proteins (MS4A) that are expressed together with a receptor guanylyl cyclase (GC-D) in a small subset of RNs in rodents (Greer et al., 2016; Juilfs et al., 1997; Leinders-Zufall et al., 2007; Figure 2B). They only have four transmembrane domains and responds to a variety of diverse odors, detecting gases, pheromones and food related odors (Greer et al., 2016; Hu et al., 2007; Leinders-Zufall et al., 2007; Munger et al., 2010). 


\section{Olfactory receptor neurons: receptor expression and signaling cascade}

\section{The one neuron - one receptor rule}

The vast number of genes encoding for single receptor proteins with different ligand specificities gives the olfactory system a predisposition to distinguish between a myriad of odorants. From this genetic baseline, the olfactory systems has several options: I) an integrative approach, were single RNs express multiple receptors and coordinate the ligand-induced responses or II) a discriminatory approach, were each sensory neuron is endowed with a single receptor, thus becoming a functional information channel directly linked to the ligand specificity of this receptor (Araneda et al., 2000; Dalton and Lomvardas, 2015).

While e.g. the bitter taste sensing system (Adler et al., 2000) and the olfactory system of $C$. elegans (Bargmann, 2006) have taken the former route, the vertebrate olfactory system is seemingly set up for maximum discriminatory power (Dalton and Lomvardas, 2015). It was first shown for the rodent ORs, that each olfactory RN expresses a single allele of a single OR gene in a seemingly stochastic manner (Chess et al., 1994; Dalton and Lomvardas, 2015; Serizawa et al., 2000, 2003; Shykind et al., 2004). In brief, the OR genes are initially silenced by epigenetic marks on the heterochromatin (Dalton and Lomvardas, 2015; Lomvardas et al., 2006; Monahan and Lomvardas, 2015). A rare coincidence of several transcriptional enhancers (Markenscoff-Papadimitriou et al., 2014; Monahan et al., 2017) then triggers the de-repression of the OR allele via demethylation (Vyas et al., 2017), which is in turn hindering the expression of another allele through negative feedback (Lewcock and Reed, 2004; Serizawa et al., 2003). As a result, each OR is expressed in RNs distributed pointilistically in a specific expression zone in the olfactory epithelium (Dalton and Lomvardas, 2015; Ressler et al., 1994; Vassar et al., 1994; Zapiec and Mombaerts, 2020). Even though almost all of the characterization of monoallelic expression has been done in mice, there is evidence supporting a similar expression mechanism in other vertebrates (Barth et al., 1997; Ferreira et al., 2014; Mori et al., 2000; Ngai et al., 2003). Monoallelic expression was also validated for rodent TAARs (Johnson et al., 2012; Liberles and Buck, 2006), V1Rs (Rodriguez et al., 1999) and FPRs (Rivière et al., 2009). However there is evidence that the exact expression mechanisms might differ from the OR-system (Dalton and Lomvardas, 2015).

The V2R family however shows a different expression pattern. Of the four V2R families described in rodents (A-D), single alleles of families $A / B / D$ have been shown to be expressed pointilistically in the VNO, while the C family (also named V2R2) are more broadly expressed (Herrada and Dulac, 1997; Matsunami and Buck, 1997; Ryba et al., 1997). It was shown that either one (Martini et al., 2001; Silvotti et al., 2007) or multiple V2R2s (Silvotti et al., 2011) are co-expressed with a monoallelically expressed member of the other $\mathrm{V} 2 \mathrm{R}$ families in a heteromeric fashion, reminiscent of the heteromeric receptors in insects (Larsson et al., 2004). A similar expression dynamic was shown in zebrafish (DeMaria et al., 2013) and inferred from experiments in Xenopus laevis (Syed et al., 2013, 2017). Many V2Rs in the rodent VNO are additionally co-expressed with components of an immune multigene family (class I major histocompatibility complex) (Ishii and Mombaerts, 2008; Leinders-Zufall et al., 
2014), which is hypothesized to increase pheromone sensitivity in the rodent VNO (Leinders-Zufall et al., 2004, 2014).

The recently described MS4A/GC-D system uses a different strategy compared to all the other vertebrate receptors and resembles more the $C$. elegans expression logic (Greer et al., 2016). Some members of the MS4A family are expressed in all RNs of this subsystem, and most RNs express multiple MS4As. In this case, the system seems to be set up to have a broader odorant recognition system, which might facilitate odor blend detection over single compound discrimination (Greer et al., 2016).

\section{$\mathrm{RN}$ types and their receptors}

Olfactory receptors are expressed on ciliary or microvillous protrusions from dendritic terminal knobs of bipolar RNs (Figure 3). The two major types are consequently named ciliated and microvillous RNs and are present in the olfactory organs of most vertebrates (Eisthen, 1992; Hansen and Zielinski, 2005; Hansen et al., 1998; Helling, 1938; Munger et al., 2009). Some additional morphologically distinct RN types have been described in fishes (crypt cells) (Hansen and Finger, 2000), and particularly in zebrafish (kappe neurons; pear shaped neurons) (Ahuja et al., 2015; Wakisaka et al., 2017). Whether these morphotypes are fish-specific remains to be elucidated. Some hybrid RNs consisting of both cilia and microvilli have been described in various lineages of reptiles and birds (Graziadei and Bannister, 1967; Hansen, 2007; Wakabayashi and Ichikawa, 2008).

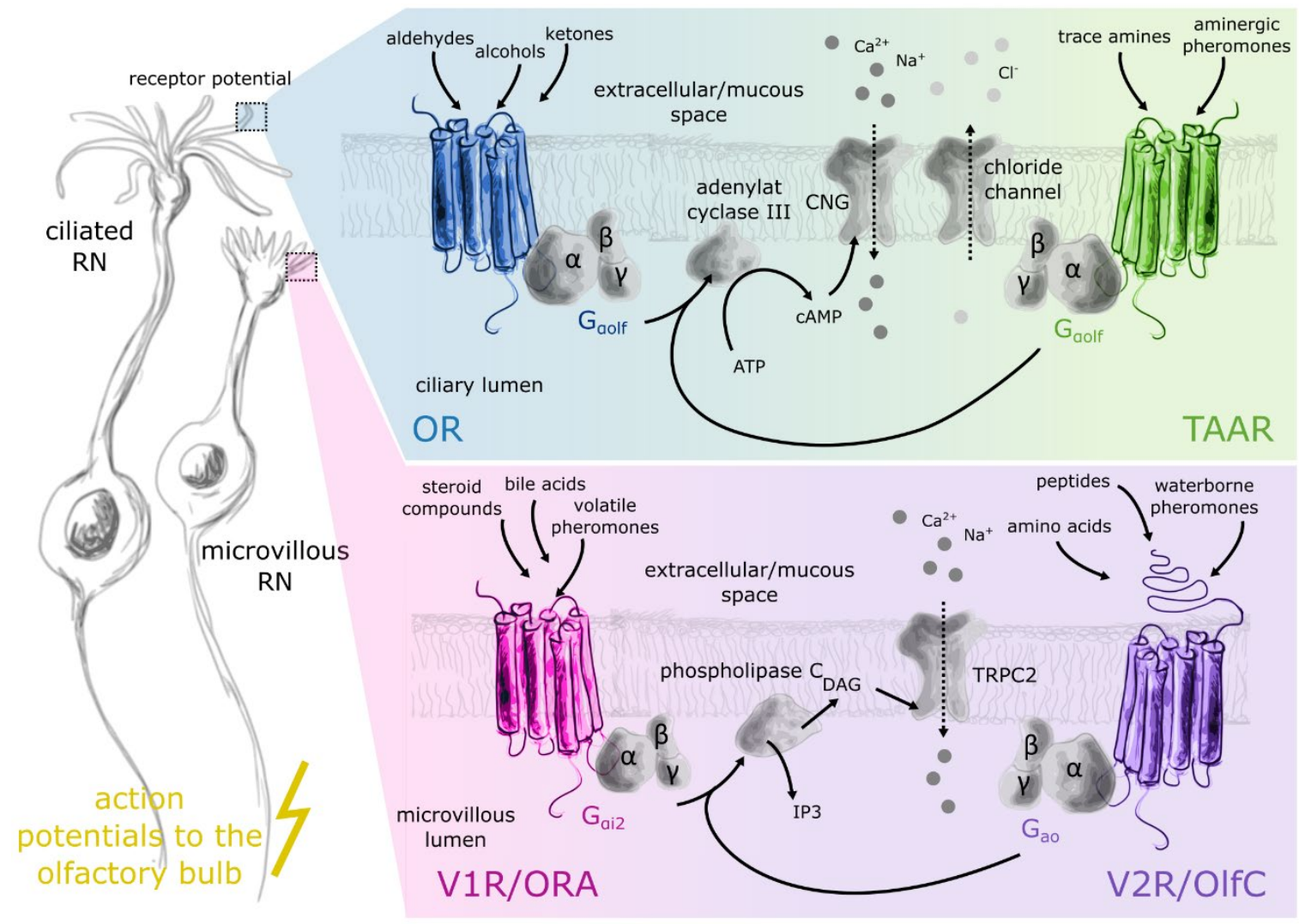


Figure 3 Types of vertebrate $\mathbf{R N}$ and their intracellular signaling pathways.

Most vertebrates have morphologically different types of RNs. Ciliated and microvillous RNs are shown. Schemes represented in this Figure are based on data mostly from rodents and considered similar in most vertebrates. Ciliated RNs in the main olfactory epithelium of rodents generally express ORs or TAARs (upper panel). Theses receptor types are linked to an intracellular signaling pathway relying on $\mathrm{G}_{\alpha o l f}$, which activates Adenylate cyclase III to generate CAMP. Increases of intracellular CAMP in turn facilitate the influx of cations and the efflux of anions. Microvillous RNs in the VNO of rodents expresses V1Rs linked to $G_{\alpha i 2}$ or V2Rs linked to $G_{\alpha o}$. The vomeronasal pathway includes a membrane bound phospholipase $C$ and a TRPC2 dependent influx of cations. Ligands shown for the various receptor types are generalized for all vertebrates. Expression of members of the remaining receptor gene families are not shown. cAMP cyclic adenosine monophosphate, ATP adenosine triphosphate, CNG cyclic nucleotide gated ion channel, DAG diacylglycerol, IP3 inositol triphosphate, OR OR-type olfactory receptor, RN receptor neuron, TAAR trace amine associated receptor, TRPC2 transient receptor cation channel C2, V1R vomeronasal type 1 receptor, V2R vomeronasal type 2 receptor.

Generally, ORs and TAARs (and MS4A in rodents) are expressed in ciliated neurons (Figure 3, upper panel) while microvillous neurons express V1Rs/ORA, V2Rs/OIfC (and FRPs in rodents; Figure 3, lower panel) (Bear et al., 2016; Manzini and Korsching, 2011; Munger et al., 2009). However, this distinction does not seem to be absolute and has been only poorly examined in non-rodent vertebrate species (Manzini and Korsching, 2011). The crypt cells, which are way less abundant than the other two types have been shown to express one gene of the ORA family (Oka et al., 2012), while the receptors linked to the other morphotypes are still elusive (Olivares and Schmachtenberg, 2019).

\section{Intracellular signaling cascades}

Long before the actual olfactory receptors were known, it was shown that RNs create electrical fields upon odorant stimulation, which they transmit to the olfactory bulb (OB) (Ottoson, 1959). Even though a huge diversity of receptors is known now, the intracellular signaling pathways are quite uniform and conserved across the vertebrate tree of life (Manzini and Korsching, 2011; Schild and Restrepo, 1998). Two major pathways have been described in detail and are seemingly employed in ciliated and microvillous RNs, respectively (Manzini and Korsching, 2011; Figure 3).

\section{cAMP pathway in ciliated RNs}

In ciliated RNs in the mouse, $\mathrm{G}_{\text {aolf }}$ (Jones and Reed, 1989) as $\alpha$-subunit of a heterotrimeric G-protein has been identified as a major player of the signal transduction cascade (Figure 3, upper panel). Lack of this G-protein subunit leads to severe anosmia in mice (Belluscio et al., 1998). Extracellular ligand binding to the GPCRs triggers the exchange from GDP to GTP in the binding domain of $\mathrm{G}_{\alpha o l f}$, which in turn dissociates from the $\beta \gamma$-dimer, activating the membrane-bound adenylate-cyclase III (ACIII) (Pace et al., 1985). ACIII converts ATP to the second messenger cAMP. Intracellular cAMP open cyclic nucleotide gated ion channels (CNG), triggering an influx of cations like $\mathrm{Na}^{+}$and $\mathrm{Ca}^{2+}$ from the external mucus (Nakamura and Gold, 1987). The crucial function of both ACIII as well as CNG have been validated by knock-out models (Brunet et al., 1996; Wong et al., 2000). The cation influx leads to the generation of a receptor potential and calcium influx mediates $\mathrm{Cl}^{-}$efflux via calcium-activated chloride 
channels (Kleene and Gesteland, 1991; Kurahashi and Yau, 1993). These currents in turn suffice to depolarize the cell and trigger action potential generation.

\section{CAMP-independent pathway in microvillous RNs}

The general signaling cascade in mammalian microvillous RNs is linked to the expression of G-proteins with $G_{\alpha o}$ and $G_{\alpha i 2}$ as $\alpha$-subunits (Figure 3 ) with a mutually exclusive expression pattern (Chamero et al., 2011; Halpern et al., 1995; Jia and Halpern, 1996). After GTP-bound $G_{\alpha o / i 2}$ dissociates, the $\beta \gamma$-dimer activates the membrane-tethered Phospholipase- $C$, which catalyzes the generation of diacylglycerol (DAG) and inositol-1-4-5-triphosphate (IP3) (Rünnenburger et al., 2002). The role of the $\alpha$-subunits $G_{\alpha o}$ and $G_{\text {aiz }}$ in downstream signaling is not entirely understood, yet their roles seem essential for proper function (Chamero et al., 2011; Norlin et al., 2003). A key player of these cAMP- independent signaling cascades is a cation channel dependent on the transient receptor potential cation channel (TRPC2) (Liman et al., 1999). TRPC2 deficient mice have shown to exhibit a quite dramatic phenotype with a severe loss of sex discrimination and other pheromone mediated behaviors like aggression (Leypold et al., 2002; Stowers et al., 2002). It was proposed that TRPC2-dependent calcium and sodium influx is activated by DAG (Lucas et al., 2003), while IP3 mediates calcium influx from intracellular storages (Yang and Delay, 2010). Higher levels of intracellular calcium subsequently amplify the receptor potential by opening chloride channels and triggering sufficient membrane depolarization for the generation of action potentials (Yang and Delay, 2010).

\section{Functional and molecular segregation of olfactory input and projections}

The molecular arsenal of highly variable receptor repertoires, different types of sensory neurons and signaling machineries feed the notion that olfaction cannot be viewed as a uniform sense. The behavioral programs linked to odors like rotten food vs. e.g. sexual attraction vary dramatically. Also, the nature of a multi-molecular pheromonal blend vs. a non-specific fruity ester molecule calls for different neuronal recognition mechanisms. Thus, the very nature of the varied chemical world dictates that the olfactory input must be ordered in what are generally described as parallel processing streams or olfactory subsystems (Figure 4).

\section{The big divide: main vs. accessory olfactory system}

Historically, a bipartition of the mammalian olfactory periphery into the main olfactory epithelium (MOE) and the VNO was first described by Jacobson at the beginning of the $19^{\text {th }}$ century (Jacobson, 1811). Since an anatomically distinct VNO was only found in terrestrial tetrapods, it was considered to be an adaptation to life on land (Bertmar, 1981). This theory proved inconclusive though, because the VNO is also present in the aquatic larvae of amphibians and features of the tetrapod VNO are already present in lower vertebrates (Eisthen, 1992). Similarly, the idea of the VNO as a pheromone-detecting apparatus in addition to the odorant-detecting MOE proved oversimplified in the light of modern neurobiology (Eisthen, 1992, 1997). As already described, the vomeronasal receptors are older than the actual tetrapod VNO, as are its main cellular components, the microvillous RNs (Grus and Zhang, 
2009). Therefore, I will look at the parallel processing streams constituted by these elements across the vertebrate tree to dissect distinct olfactory functions (Figure 4).

Both the tetrapod MOE and VNO as well as the not segregated olfactory epithelium (OE) in lower vertebrates follow a general organizational blueprint. On the cellular level, the RNs are complemented by sustentacular cells, which fulfill a supporting, glial-like function, and stem-cells or basal calls, which are key players for the regenerative capacity of the olfactory (Schwob, 2002; Schwob et al., 2017). Their functions are largely reviewed elsewhere and will not be discussed in detail here. Within the epithelium, RNs expressing the same receptors are distributed in a mosaic-like fashion and intermingled with different populations of RNs (Mombaerts et al., 1996; Ressler et al., 1994; Vassar et al., 1994). In a remarkable process of molecular sorting and axonal guidance, axons of RNs expressing the same allele of olfactory receptor converge on few common target structures in the OB called glomeruli (Feinstein and Mombaerts, 2004; Mombaerts, 2006; Ressler et al., 1994; Vassar et al., 1994). Glomeruli consist of the terminal branches of the RNs, and dendritic arborizations of local interneurons and projections neurons, which in turn project axons to higher brain centers (Bear et al., 2016; Nagayama et al., 2014). The exact connectivity scheme between the RN axons, the number of innervated glomeruli and the connection scheme of projection neuron dendrites to glomeruli varies according to species and olfactory subsystem and will thus be covered differentially (Figure 5).

\section{Fishes: An all in one approach to olfaction}

Fishes generally have a single olfactory organ on each side of the head, consisting of several folds or lamellae lined with olfactory epithelium (Hamdani and Døving, 2007; Olivares and Schmachtenberg, 2019). In most extant species of the jawless cyclostomes, the left and right nostril are fused at the midline, a feature that is believed to be derived in the cyclostomes and not ancestral to vertebrates (Kleerekoper and Erkel, 1960). The presence of both microvillous and ciliated RNs in the hagfish (Theisen, 1973) as well as multi-morph RNs in the sea lamprey Petromyzon marinus (Laframboise et al., 2007) support the idea that a putative distinction of parallel processing streams might be a very basal vertebrate feature (Eisthen, 1992). In P. marinus, an accessory olfactory organ present in tubular diverticula in the olfactory sac has been described already in 1887 (Scott, 1887). More recently, RNs in this cryptic organ of the lamprey have been described as cuboid and ciliated (Ren et al., 2009), expressing the same receptor genes as the rest of the lamprey olfactory organ (ORA, OR, TAAR-like) (Chang et al., 2013). The accessory RNs project their axon towards a glomerular target in the medial regions of the paired $O B$ and constitute a pathway independent of $G_{\alpha o l f} . G_{\alpha o l f}$-positive RNs instead project to the non-medial glomeruli in the OB (Frontini et al., 2003; Ren et al., 2009). Physiological stimulation experiments showed that the medial $O B$ region reacts to both amino acids as well as steroid bile acids and lamprey pheromones, while the lateral glomeruli predominantly showed responses to amino acids (Green et al., 2017). The lateral stream has been associated with feeding behavior (Li et al., 1995; Sorensen and Caprio, 1998). The medial processing stream on the other hand seems to be involved in homing and mating related behavior (Daghfous et al., 2016; Derjean et al., 2010; Green et al., 2013; Li et al., 1995; Sorensen et al., 2005). Even though homology between the 
accessory organ in lampreys and the tetrapod VNO is widely disputed, the segregation of olfactory function seems to be a basal requirement in vertebrates.

While the lamprey seems to mostly rely on OR, V1R/ORA and TAAR-like related odor processing and the ciliated morphotype of RNs, olfaction in cartilaginous fishes is based almost entirely on the expression of V2Rs in microvillous RNs (Ferrando and Gallus, 2013; Howard et al., 2013; Sharma et al., 2019). Cartilaginous fishes have been shown to lack ciliated RNs and their $G_{\text {aolf-related signaling }}$ cascade (Ferrando et al., 2009, 2017), relying on $G_{\alpha o}$ instead (Ferrando et al., 2009). Even though these fishes seemingly possess only a small and not variated receptor repertoire (Sharma et al., 2019; Venkatesh et al., 2014) they are generally believed to rely quite heavily on their sense of smell for orientation and foraging (Yopak et al., 2015). In comparison to most other vertebrates, a somatotopic organization has been proposed for the shark olfactory system, instead of a functional chemotopic organization (Meredith et al., 2013). In its general reliance on microvillous RNs, $G_{\alpha o}$ and V2Rs, the olfactory system of sharks resembles more the mammalian VNO than the MOE, but the functional implications of the lineage specific loss of ciliated RNs remains unclear (Ferrando and Gallus, 2013).

A substantial amount of data is available on the olfaction in teleost fish species (Hamdani and Døving, 2007; Olivares and Schmachtenberg, 2019). Their olfactory organ consists of a varying number of lamellae organized in an olfactory rosette lined by a pseudostratified sensory epithelium (Hamdani and Døving, 2007; Hansen and Zielinski, 2005; Olivares and Schmachtenberg, 2019; Figure 4, upper panel). Crypt cell somata are located in the uppermost layer, followed by microvillous and ciliated RNs (Eisthen, 1992; Hansen and Zielinski, 2005; Figure 4, upper panel, left inset).

Ciliated RNs were found to express OR-type receptors (Ngai et al., 2003) or TAARs (Dieris et al., 2017; Hussain et al., 2009, 2013) linked to $G_{\text {aolf }}$ (Bazáes et al., 2013; Hansen et al., 2003, 2004; Sato, 2005). Odorant responsiveness of ciliated RNs seems to be extremely diverse. In zebrafish, an OR-mediated response to prostaglandin F2 was shown to induce male courtship behavior (Yabuki et al., 2016), and TAAR13c was demonstrated to be responsive to cadaverine, a polyamide related to decaying food or a dead conspecific (Dieris et al., 2017; Hussain et al., 2013). In the channel catfish, ciliated neurons showed responses both to bile acids as well as amino acids (Hansen et al., 2003), which are generally believed to be social cues (Buchinger et al., 2014, 2019) or food odors, respectively (Sorensen and Caprio, 1998). Generally, the ciliated RN population dispersed in the OE projects to a set of glomeruli that is distinct of the projection target of microvillous RNs (Bazáes et al., 2013) and is located more medially in most fishes (Braubach et al., 2012; Friedrich and Korsching, 1998a; Hamdani, 2002; Hansen et al., 2003; Sato, 2005; Figure 4, upper panel). 

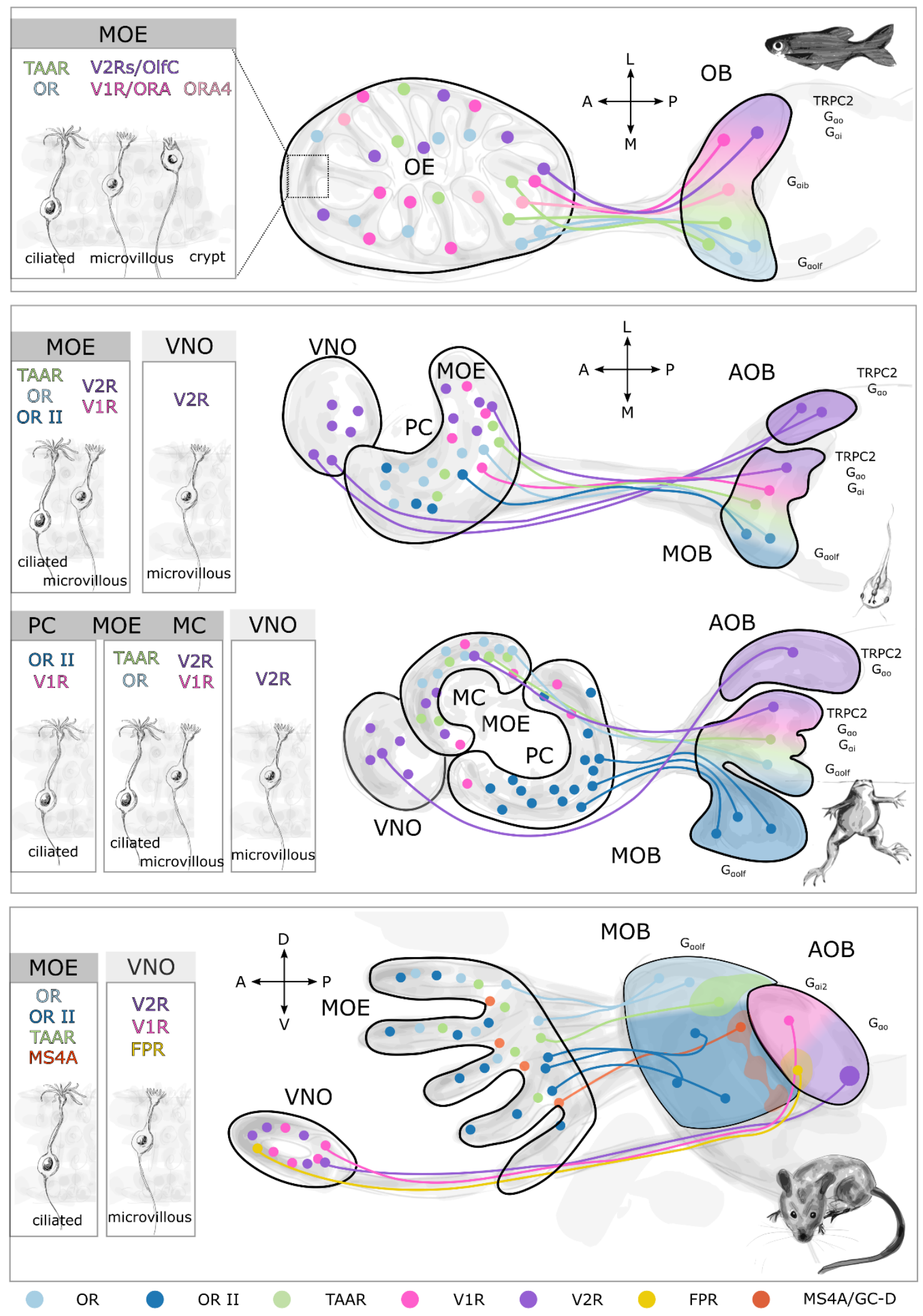
Figure 4 Subsystem segregation in vertebrate olfactory systems.

The schematic summarizes olfactory subsystems in the zebrafish (upper panel), larval and adult clawed frogs (middle panel) and the mouse (lower panel). The boxes on the left represent the morphological types of RNs and their receptors expressed in the various olfactory organs. The scheme is simplified, and some receptor expression data is inferred based on similarity to other species. Only one side of the olfactory systems is shown. While the fish olfactory system consists of a single peripheral organ, the clawed frog and the mouse have a clearly segregated VNO-AOB pathway. Vomeronasal receptors in the mouse are clearly restricted to the VNO, while they are intermingled with the other receptor families in the fish and the frog. The smaller subsystems of the mouse (Grüneberg Ganglion and Septal organ) are not shown. The MOE of the adult clawed frog can be subdivided into a middle cavity $(\mathrm{MC})$ and a principal cavity $(\mathrm{PC})$, which are associated with aquatic and aerial olfaction, respectively, and innervate distinct zones of the $\mathrm{MOB}$. The depicted schemes are mainly based on the following publications and reviews (Bear et al., 2016; Bozza et al., 2009; Braubach et al., 2012; Manzini and Schild, 2010; Sato, 2005). A anterior, AOB accessory olfactory bulb, D dorsal, FPR formyl peptide receptor, GC-D receptor guanyly cyclase , L lateral, M medial, MC middle cavity, MOB main olfactory bulb, MOE main olfactory epithelium, MS4A membrane-spanning 4A receptor, OB olfactory bulb, ON olfactory nerve, OR OR-type olfactory receptor, OR II mammalian class II/ $\gamma$ subfamily of ORs, ORA olfactory receptor related to class A GPCRs, P posterior, PC principal cavity, type D, TAAR trace amine associated receptor, $V$ ventral, V1R vomeronasal type 1 receptor, V2R vomeronasal type 2 receptor, VNO vomeronasal organ.

Microvillous RNs on the other hand express mainly V2Rs/OlfC receptors (Ahuja et al., 2018; Cao et al., 1998; Speca et al., 1999) and are tuned particularly to amino acids (Koide et al., 2009; Sato and Sorensen, 2018; Speca et al., 1999). In contrast to the single G-protein found in their ciliated counterparts, the microvillous RNs express either $G_{\alpha 0}, G_{\alpha i}$ or $G_{\alpha q}$, possibly constituting different subtypes (Belanger et al., 2003; Hansen et al., 2003, 2004). Microvillous RNs generally project to laterally located glomerular clusters in the OB (Friedrich and Korsching, 1998b; Hamdani, 2001a; Hansen et al., 2003; Hara and Zhang, 1997; Sato, 2005; Figure 4, upper panel). Projection neurons connected to the lateral cluster in the $\mathrm{OB}$ projected to higher brain centers via the lateral olfactory tract, which was found to be involved in feeding behavior in the crucian carp (Hamdani, 2001a, 2001b).

Crypt cells form the third population of RNs present in most fish species (Figure 4 upper panel). In zebrafish, nearly all crypt cells have been found to express only a single receptor gene of the ORA gene family and the inhibitory $\mathrm{G}_{\alpha i 1 b}$ (Oka et al., 2012). In other fishes, different G-protein $\alpha$ subunits seem to be expressed in the crypt cell population (Belanger et al., 2003; Hansen et al., 2003, 2004). Crypt cells in the mackerel have been reported to respond to food-related amino acids (Vielma et al., 2008), while evidence in carps points to a possible importance as sex pheromone detectors (Bazáes and Schmachtenberg, 2012; Hamdani and Døving, 2006; Schmachtenberg, 2006). Crypt cells in the zebrafish were shown to converge onto a single medio-dorsal glomerulus, which makes it an extreme case of a labelled line consisting of a single receptor gene, a unique morphotype of RN and a single glomerular relay station in the OB (Ahuja et al., 2013). Similarly, the $\mathrm{G}_{\alpha o}$-dependent kappe neurons found only in zebrafish so far also exclusively converge onto a single medio-dorsal glomerulus of unknown function (Ahuja et al., 2015) and an adenosine-receptor mediates attractive stimuli via pear shaped neurons projecting to a single lateral glomerulus (Wakisaka et al., 2017). 
The extensive body of knowledge on fish olfaction demonstrates that anatomical segregation into several olfactory surfaces is not required to develop parallel and differential odor processing. The first segregated putative VNO primordial was described in a lungfish species (González, 2010; Nakamuta et al., 2012; Wittmer and Nowack, 2017). This organ was found in epithelial crypts and consists of $G_{\alpha 0^{-}}$ positive microvillous RNs (González, 2010; Nakamuta et al., 2012), while the rest of the lamellar OE included both $\mathrm{G}_{\alpha o l f}$-positive ciliated and $\mathrm{G}_{\alpha o}$-positive microvillar RNs. Additionally, a primordial $A O B$ and further accessory pathways to the medial amygdala were identified (González, 2010; Nakamuta et al., 2012). Nakamuta and colleagues hypothesized the expression of V2Rs, which would highlight its possible homology to the amphibian VNO (Nakamuta et al., 2012).

\section{Tetrapods: Anatomical segregation of subsystems}

Amphibians are arguably the most interesting vertebrates to examine adaptations necessary for terrestrial and aquatic olfaction. They were the first emerging land living vertebrates and most species of modern amphibia combine an aquatic larval stage with an adult that lives more or less independently from the water (Duellman and Trueb, 1994; Wells, 2007). The clear anatomical separation of the VNO from the MOE seems to be ancestral to all three lineages of the extant amphibians, the caecilians, the urodeles/salamanders and the anura/frogs and toads (Eisthen, 1992, 1997; Saint Girons and Zylberberg, 1992; Schmidt and Wake, 1990) with secondary loss of the VNO in proteid salamanders (Eisthen, 2000). Nevertheless, the vomeronasal receptors are not completely restrained to the VNO, as it is in most mammals. Amphibians thus show an incomplete segregation of the main and vomeronasal pathways (Date-Ito et al., 2008; Eisthen, 1992; Syed et al., 2013).

The common configuration of the olfactory periphery in adult caecilians and salamanders consists of microvillous RNs arranged in the VNO and both microvillous as well as ciliated RNs in the MOE (Eisthen, 1992; Eisthen et al., 1994; Saint Girons and Zylberberg, 1992; Różański and Żuwała, 2019; Figure 4, middle panel). The adult anuran olfactory organ is generally more complex (Figure 4, middle panel), exhibiting an interconnected tripartite chamber system (Helling, 1938; Reiss and Eisthen, 2008). The inferior diverticulum harbors the VNO, which consists of only microvillous RNs, while the medial diverticulum or principal cavity (PC) consists of only ciliated RNs (Bloom, 1954; Eisthen, 1992; Föske, 1934; Mair et al., 1982; Menco, 1980). Since the VNO operates in an aquatic medium, while the PC epithelium is usually filled with air, it seems plausible to assume their respective roles as 'water'- vs 'air-nose'. However, most adult anurans have an additional olfactory epithelium, a separated patch of epithelium in the antero-ventral region of the PC called the recessus olfactorius (Helling, 1938; Nowack et al., 2013; Reiss and Eisthen, 2008). This recessus lacks the mucus-producing Bowman's glands (Getchell and Getchell, 1992) and consists of both microvillous and ciliated RNs (Nowack et al., 2013). The same epithelial composition is observed in the lateral diverticulum or middle cavity (MC) of the secondarily aquatic Xenopus (Hansen et al., 1998; Manzini and Schild, 2010; Figure 4, middle panel) and the anterior part of the PC epithelium in Ascaphus truei, one of the earliest diverging anurans (Benzekri and Reiss, 2012). Strikingly, the cellular composition of these epithelia are also identical to the blueprint for the MOE of larval anurans (Benzekri and Reiss, 2012; Manzini and Schild, 2010; Reiss and Eisthen, 2008; Figure 4, middle panel). Even though a homology of these diverse adult epithelia is 
disputed (Helling, 1938; Nowack et al., 2013), they seem to share the function of an additional 'waternose'.

Only little data is available on the distribution of olfactory receptors or the intracellular signaling machinery in amphibians. OR-type receptors have been found to be distributed in distinct expression zones in the MOE of the tiger salamander (Marchand et al., 2004), putatively responding to small volatile odorants like alcohols, ketones and aldehydes (Kauer, 2002). V2Rs have been identified to be distributed in the VNO of the red-legged salamander (Kiemnec-Tyburczy et al., 2012). Microvillous RNs of the salamander VNO seem to be dependent on $G_{\alpha o}$ (Nakada et al., 2014) and trpc2 (KiemnecTyburczy et al., 2012), and project to glomeruli in the AOB (Eisthen et al., 1994). However some instances of $G_{\text {aolf }}$ expression in the VNO of urodeles are reported (Kiemnec-Tyburczy et al., 2012; Nakada et al., 2014) and some RNs in the MOE also project to the AOB, showing an even more incomplete separation of the main and accessory system (Nakada et al., 2014).

Most data concerning the anuran olfactory system comes from the genus Xenopus (Manzini and Schild, 2010; Figure 4, middle panel). Xenopus permanently lives in the water (Kramer, 1933), however episodical overland movements on the search for water bodies or food are reported (Measey, 2016; Du Plessis, 1966). Microvillous RNs in the VNO of Xenopus express V2Rs (Hagino-Yamagishi et al., 2004; Syed et al., 2013) and rely on $G_{\alpha o}$ and TRPC2 for signal transduction, similar to the salamander VNO (Gliem et al., 2013; Kiemnec-Tyburczy et al., 2012; Sansone et al., 2014). The terrestrial Bufo japonicus instead seems to have a bimodal VNO, with cells expressing $G_{\alpha_{0}}$ or $G_{\alpha o l f}$ as alpha subunits (HaginoYamagishi and Nakazawa, 2011), a feature that is unique among all anurans examined to date (Gliem et al., 2013; Hagino-Yamagishi et al., 2004; Jungblut et al., 2009). Xenopus V2Rs are however not restricted to the VNO, since an ancient clades of V2Rs are also expressed in the MC epithelium (Syed et al., 2017). In contrast to mammals, the small V1R-family of the frogs is not expressed in the VNO but the MOE of Xenopus (Date-Ito et al., 2008; Gliem et al., 2013). OR genes belonging to the subfamily which expanded in terrestrial vertebrates have been found to be preferentially expressed in the PC epithelium, which supports the idea of the PC as the adult 'air-nose' of Xenopus (Freitag et al., 1995, 1998a; Figure 4, middle panel).

In the larval MOE ORs, TAARs, V1Rs and some V2RS are intermingled in the MOE, while the VNO only expresses V2Rs (Gliem et al., 2013; Mezler et al., 1999, 2001; Syed et al., 2013, 2017; Figure 4, middle panel). Axonal projections to the $\mathrm{MOB}$ can be coarsely segregated into two processing streams: the lateral cluster of glomeruli is cAMP-independent, mostly amino acid-sensitive (Gliem et al., 2013; Manzini and Schild, 2003; Syed et al., 2017) and probably mediated by microvillous RNs expressing V1Rs/V2Rs (Gliem et al., 2013; Syed et al., 2017). The medial glomeruli are instead $\mathrm{G}_{\text {aolf-positive, and }}$ form part of a putatively ciliated, primarily OR-related and cAMP-dependent processing stream (Gliem et al., 2013; Manzini et al., 2007).

Research concerning amphibian olfaction was initially focused on chemical communication mainly in salamanders (Houck, 2009; Woodley, 2015). Salamanders and newts display chemically-guided mating behaviors, while frog courtship is mostly believed to rely on auditory cues (Kelley, 2004). Several male peptide pheromones from urodeles were isolated (Kikuyama et al., 1995; Rollmann et al., 1999; 
Yamamoto et al., 2000) and shown to be detected by VNO cells, which fed the notion of the VNO as the main center for pheromonal processing (Iwata et al., 2013; Toyoda et al., 1999; Wilburn et al., 2017; Wirsig-Wiechmann et al., 2006). However, in Ambystoma mexicanum, both the VNO as well as the MOE are involved in finding suitable mating partners and assessing their reproductive state (Park et al., 2004). Similarly, mating related cues were detected by both olfactory organs in Notophtalmus viridescence (Park and Propper, 2002). The exact receptors mediating these pheromonal responses in salamanders are not deorphanized yet and even less is know from anurans. A single waterborne sex pheromone is known from the magnificent tree frog, yet no receptors involved are known (Wabnitz et al., 1999). In Xenopus sulfated steroids have been shown to be strong vomeronasal cues, however these stimuli also triggered responses in the MOE (Sansone et al., 2015).

While the anatomical segregation into the main and accessory olfactory system of the amphibians is present throughout their life stages, the presence and size of a segregated VNO varies in amniotic vertebrates. The most recent common ancestor of the sauropsids (reptiles and birds) is generally assumed to be terrestrial, and all major clades of reptiles contain secondarily aquatic members (Thewissen and Nummela, 2008). Similar to the anurans, turtles have multiple olfactory chambers lined with sensory epithelia, which putatively serve to smell both in water and air (Thewissen and Nummela, 2008) and project to distinct parts of the glomerular array in the olfactory bulb (putatively MOB and AOB) (Nakamuta et al., 2016a) The 'water-nose' is sometimes described as the turtle VNO and has been shown to consist of mostly microvillous RNs that often co-express both $G_{\alpha o l f}$ and $G_{\alpha o}$ (Nakamuta et al., 2016a; Wakabayashi and Ichikawa, 2008). Nonetheless, many inter-species differences have been reported (Kondoh et al., 2019; Nakamuta et al., 2016b). The turtle VNO responds to a very broad spectrum of odorants (Hatakana and Matsuzaki, 1993), which implies a variety of different receptor types. The turtle 'air-nose' interestingly exhibits a hybrid morphotype of RNs bearing both cilia as well as microvilli (Eisthen and Polese, 2007). This type of RNs has been described in the OE of the alligator (Hansen, 2007), several bird species (Eisthen, 1992; Eisthen and Polese, 2007; Graziadei and Bannister, 1967) and a lizard species (Kratzing, 1975), suggesting a more important role in sauropsid olfaction. Since there is no VNO in adult crocodilians and birds, the MOE is the sole responsible organ for olfaction in these species (Eisthen and Polese, 2007).

Inversely to the evolutionary loss of the VNO in the crocodile-bird lineage, olfaction in snakes seems to be predominantly mediated by the well-developed VNO (Dawley, 1998; Eisthen and Polese, 2007). The MOE and the VNO of the garter snake have been found to consist of ciliated and microvillous RNs (Wang and Halpern, 1980b, 1980a) and express $G_{\alpha o l f}$ and $G_{\alpha o} / G_{\alpha i}$ respectively (Kondoh et al., 2013; Luo et al., 1994). RNs in the VNO express members of an enlarged V2R gene family (Brykczynska et al., 2013). Sex pheromones (Huang et al., 2006), but also prey-related odors (Jiang et al., 1990) are transported to the VNO via tongue-flicking behavior. This behavior has also been found in fully aquatic sea snakes (Kishida et al., 2019; Thewissen and Nummela, 2008). While the main olfactory system seems to degenerate, the VNO constitutes the putatively sole mediator of olfaction in these animals (Kishida et al., 2019). 
The sister clade of the sauropsids only consists of a single extant animal group, the mammals. The plesiomorphic morphology of RNs in the MOE seems to be ciliated, while the VNO is composed of solely microvillous RNs (Eisthen, 1992; Munger et al., 2009). Except for some marine mammals like whales (Kishida et al., 2015) or manatees (Mackay-Sim et al., 1985), which possess only a vestigial MOE, the MOE of most mammals is functional. Based on the diverse ecological niches occupied by mammals, considerable variation in their olfactory systems is expectable (Salazar and Sánchez Quinteiro, 2009), however available data is mostly limited to rodents. Ciliated RNs in the rodent MOE mainly express OR-type olfactory receptors of both mammalian class I and II (Buck and Axel, 1991) in overlapping expression zones (Bozza et al., 2009; Miyamichi et al., 2005; Vassar et al., 1993; Zapiec and Mombaerts, 2020; Figure 4, lower panel). Less abundantly, TAAR expressing RNs (Liberles and Buck, 2006), microvillous RNs expressing a type of TRP channel (Lin et al., 2007, 2008) and MS4-expressing RNs (Greer et al., 2016) are found in the MOE. Ciliated neurons in the MOE have been found to express the canonical $\mathrm{G}_{\text {aolf }}$ subunit and have a cAMP-dependent signaling cascade (Jones and Reed, 1989; Munger et al., 2009).

RNs expressing the same receptor allele have been found to project their axons to one or two glomeruli in the MOB (Mombaerts, 2006; Mombaerts et al., 1996; Ressler et al., 1994; Vassar et al., 1994). Each glomerulus thus can be considered an individual information unit for specific molecular odorantfeatures since it only receives input from a single receptor (Mori et al., 2006). In addition, projections from the various receptor families/types project to contingent glomerular domains in the MOB, forming a mostly non-overlapping topographic map (Bozza et al., 2009; Johnson et al., 2012; Mombaerts et al., 1996; Pacifico et al., 2012; Ressler et al., 1994; Vassar et al., 1994; Figure 4, lower panel). The MS4-expressing RNs instead project to a chain of interconnected glomeruli at the border between $\mathrm{MOB}$ and $\mathrm{AOB}$, termed necklace glomeruli (Greer et al., 2016; Figure 4, lower panel). Based on the variety of receptors expressed in the MOE, it is no surprise that its functions are quite diverse, including spoiled food aversion (Kobayakawa et al., 2007), predator avoidance (Ferrero et al., 2011), mating pheromone detection (Bear et al., 2016; Li, 2018; Lin et al., 2007) or $\mathrm{CO}_{2}$-sensing (Hu et al., 2007). In addition, two smaller, anatomically distinct olfactory epithelia have been described in rodents, the septal organ of Masera (Rodolfo-Masera, 1943) and the Grüneberg Ganglion (Grüneberg, 1973). Their RNs also project into distinct glomeruli within the MOB (Lèvai and Strotmann, 2003; Roppolo et al., 2006) and constitute possibly non-redundant functional subsystems in rodents (Munger et al., 2009).

The MOE plays a prominent sensory role for most mammalian species. The functionality of the VNO on the other hand varies substantially and has degenerated independently in some bat species (Bhatnagar and Meisami, 1998; Meisami and Bhatnagar, 1998; Yohe et al., 2017, 2018), marine mammals (Kishida et al., 2015; Mackay-Sim et al., 1985; Thewissen and Nummela, 2008) and old world monkeys and apes (Bhatnagar and Meisami, 1998). The extensive data from rodents shows two major populations of microvillous RNs in the VNO. RNs with more apically located somata express single alleles of the V1R family and $G_{\text {ai2 }}$ (Berghard et al., 1996; Dulac and Axel, 1995) while RNs with more basally located somata express V2Rs and $G_{\alpha o}$ (Herrada and Dulac, 1997; Matsunami and Buck, 1997; Ryba et al., 1997). In contrast to the main olfactory system, all RNs expressing the same VR allele 
innervate between 4 and 30 distinct glomeruli in the AOB (Belluscio et al., 1999; Rodriguez et al., 1999). In the $A O B$, an anterior $V 1 R / G_{\alpha i 2}$ and a posterior $V 2 R / G_{\alpha 0}$-innervated glomerular domain have been identified in rodents (Jia and Halpern, 1996; Figure 4, lower panel) and lagomorphs (Villamayor et al., 2018). The existence of this dual vomeronasal processing stream has been considered an ancestral mammalian trait, since it was also described in an early diverging marsupial and an early placental mammal (Halpern et al., 1995; Suárez et al., 2009). However the $V 2 R / G_{\alpha o}$ pathway is absent in most mammals (Barrios et al., 2014; Suárez et al., 2011; Takigami, 2000; Takigami et al., 2004; Tomiyasu et al., 2017). In rodents, an additional subsystem mediated by the FPRs has been identified in the VNO (Liberles et al., 2009; Rivière et al., 2009), which is associated with another cluster of AOB glomeruli (Dietschi et al., 2013; Figure 4, lower panel). Mammalian VNO research has been particularly focused on its involvement in detecting species-specific urine-cues or glandular pheromones, but the VNO also e.g. responds to heterospecific cues from predators (Ben-Shaul, 2015; Isogai et al., 2011; Mohrhardt et al., 2018).

The long evolutionary parallel existence between cellular components belonging to the main and the accessory olfactory systems suggests that both are vital to most vertebrates. Even though the two systems show substantial functional overlap in terms of stimulus detection, stimulus processing differs and might result in their non-redundant functionality.

\section{Evolutionary differences in the olfactory bulb circuit}

One evolutionarily constant feature of the vertebrate odor processing network in the $\mathrm{OB}$ are the glomeruli (Hildebrand and Shepherd, 1997; Strausfeld and Hildebrand, 1999). Even though they are of variable shapes and sizes in different vertebrate species or olfactory subsystems, they share a general input-output structure (Figure 5). The spheroid glomerular structures are composed of RN axon terminals forming synapses with the dendrites of several projection neurons, which in turn project their axons to a variety of higher brain centers. Additionally, different types of interneurons form synapses with the other components in the glomeruli and interfere with odor processing via inhibitory or modulatory synapses (Firestein, 2001; Nagayama et al., 2014). However, several components of the $O B$ circuit vary across vertebrates and the lack of comparative studies makes it difficult to predict, whether the differences in circuit architecture have immediate effects on olfactory perceptions (Eisthen, 1997; Eisthen and Polese, 2007).

A general evolutionary trend is an increasing degree of laminar organization in the $O B$ and a clear spatial segregation of different neuronal subtypes (Eisthen and Polese, 2007; Nieuwenhuys, 1966). In the early diverging sea lamprey, somata of projection neurons are mostly intermingled within the glomeruli and extend one or multiple primary dendrites towards mostly a single glomerulus (Figure 5A), where they form extensive arborizations (Dryer and Graziadei, 1994; Green et al., 2013; Iwahori et al., 1987). Similarly, the few examined cartilaginous fishes exhibit uni-glomerular projection neurons in close proximity to the glomerular neuropil (Dryer and Graziadei, 1993, 1994). These projection neurons solely have primary dendrites that receive direct glomerular input, while non-glomerular 
lateral dendrites are lacking. More caudally, the somata of inhibitory interneurons, the granule cells, are located (Nieuwenhuys, 1966). They have been shown to extend several dendrites to multiple glomeruli, putatively facilitating cross-talk between individual glomeruli (Dryer and Graziadei, 1994).

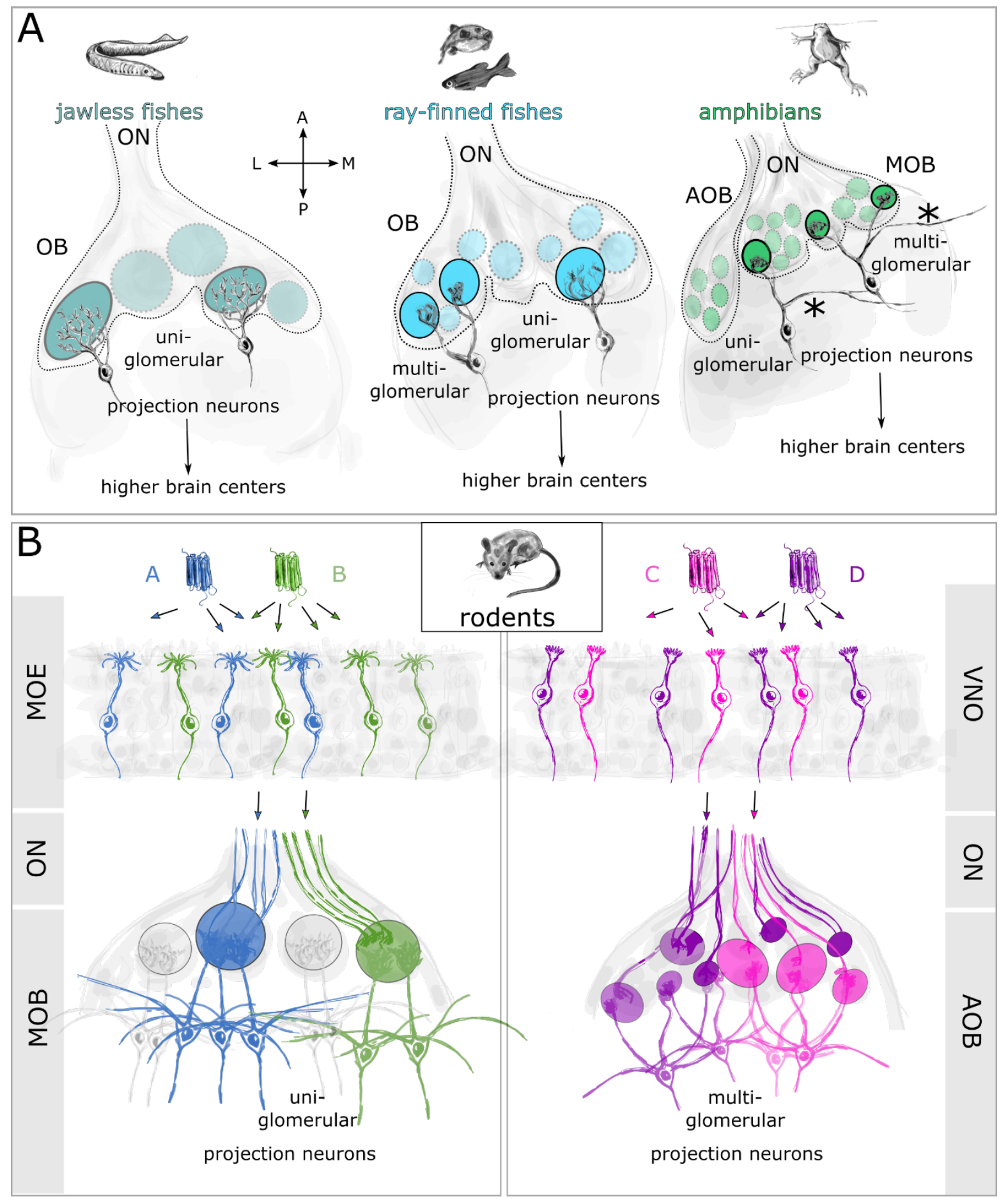


Figure 5 Glomerular wiring in the vertebrate $O B$.

A) Representative schematics of one OB hemisphere of jawless fishes, ray finned fishes and anurans are shown. Glomeruli are highlighted by dotted circles. Projection neurons are generally connecting to a single glomerulus in the jawless fish, while both uni- and multi-glomerular projection neurons are reported from ray finned fishes and amphibians. Amphibian projection neurons are distinct from the fish projection neurons by the presence of lateral dendrites (asterisks). B) Glomerular wiring in the $\mathrm{MOB}$ (left) and the AOB (right) of rodents. Individual RNs in the rodent $\mathrm{MOE}$ and $\mathrm{VNO}$ express one allele of a single olfactory receptor gene $(A$ and $B)$ or vomeronasal receptor gene ( $C$ and $D$ ), respectively. In the main olfactory system, all RNs expressing the same allele project their axons to two glomeruli in the MOB. Each projection neuron (mitral/tufted cells) connects to a single glomerulus. In the accessory olfactory system, all RNs expressing the same vomeronasal receptor allele project to multiple glomeruli and each projection neurons gets input from multiple glomeruli. Data is based on the following publications (Dryer and Graziadei, 1994; Mombaerts et al., 1996; Del Punta et al., 2002b; Ressler et al., 1994; Rodriguez et al., 1999; Vassar et al., 1994). A anterior, AOB accessory olfactory bulb, L lateral, M medial, MOB main olfactory bulb, MOE main olfactory epithelium, OB olfactory bulb, ON olfactory nerve, $\mathrm{P}$ posterior, VNO vomeronasal organ.

In ray-finned fishes, the circuit architecture seems to differ between species. Distinct layers are generally ill-defined (Satou, 1990) and some fishes like the zebrafish exhibit mostly uni-glomerular projection neurons (Fuller et al., 2006; Figure 5A), others have multi-glomerular projection neurons (Oka, 1983; Figure 5A). With the emergence of the first tetrapods, the segregation of distinct horizontal layers becomes more apparent. The primary dendritic branches of the projection neurons in amphibians are generally longer than in fishes and exhibit lateral non-glomerular branches (asterisks, Figure 5A) that give rise to the external plexiform layer (Nezlin et al., 2003; Nieuwenhuys, 1966; Scalia et al., 1991a). Single projection neurons connect to one or more glomeruli (Figure 5A) and some authors also distinguished different types of projection neurons based on their distance from the glomeruli (Dryer and Graziadei, 1994; Herrick, 1924; Jiang and Holley, 1992a; Scalia et al., 1991a). Like in fishes, the inhibitory granule cell somata are located caudally to the projection neuron somata, in the granule cell layer. In contrast to their fish counterparts, they form synapses with the lateral, nonglomerular dendrites of the projection and barely reach into the glomerular neuropil (Dryer and Graziadei, 1994). In the interglomerular space, some periglomerular interneurons were also described in both the MOB and the AOB of amphibians (Nezlin and Schild, 2000; Nezlin et al., 2003; Nieuwenhuys, 1966). Similarly, reptile projection neurons are mostly multi-glomerular and bear lateral dendrites in both the MOB (Garcia-Verdugo et al., 1986; Iwahori et al., 1989) as well as the AOB (Iwahori et al., 1989; Llahi and García-Verdugo, 1989).

The rodent $\mathrm{MOB}$ shows a well-organized layering and the glomerular neuropil is delineated by a high number of variable cells that are usually grouped together as juxtaglomerular cells (Nagayama et al., 2014; Pinching and Powell, 1971). The projection neurons are segregated into the tufted cells and the mitral cells, which are located closer and further from the glomeruli, respectively (Macrides and Schneider, 1982; Nagayama et al., 2014). Both cell types only connect to a single glomerulus (Figure 5B, left panel) and have multiple lateral dendrites (Orona et al., 1984), but have different axonal target structures and putatively different functions (Nagayama, 2010). In contrast, the AOB glomeruli are 
generally smaller and less clearly delineated. Mitral and tufted cells are not distinguished (Larriva-Sahd, 2008; Mohrhardt et al., 2018; Takami and Graziadei, 1991). Their dendritic configuration is more reminiscent of the reptile/amphibian projection neurons, since they connect to up to 10 distinct glomeruli (Larriva-Sahd, 2008; Takami and Graziadei, 1991; Yonekura and Yokoi, 2008; Figure 5B, right panel).

In the rodent $\mathrm{MOB}$, all RNs expressing the same allele of a single olfactory receptor gene converge onto one (or two) glomerulus in the MOB (Figure 5B, left panel). The information encoded by the glomerulus is then transmitted to the population of projection neurons that connect to it via their uniglomerular dendrite. This forms a single unit all dependent on the odor features detected by a single receptor protein (Figure 5B, left panel). However, in the AOB, all RNs expressing the same receptor allele converge onto multiple glomeruli, and each projection neuron receives input from multiple glomeruli (Figure 5B, right panel). It is not clear yet, whether the information received at the various dendritic branches all originate from homotypic glomeruli (Del Punta et al., 2002b) or if they integrate between different glomerular information channels (Wagner et al., 2006). To complicate things further, some of axons in the rodent $A O B$ were shown to bifurcate, terminating in multiple glomeruli (Larriva-Sahd, 2008), violating the general idea that individual RN axons are unbranched before entering a single glomerulus (Klenoff and Greer, 1998; Mombaerts, 2006). A similar pattern was observed in both the MOB as well as the AOB of Xenopus laevis (Hassenklöver and Manzini, 2013; Nezlin and Schild, 2005). Data of most other animal groups are missing in regard. It remains elusive, whether a certain olfactory wiring scheme facilitates a certain olfactory function, if wiring patterns correlate with ligands or specific environments, or if they are evolutionary strategies of specific vertebrate lineages.

\section{Objectives and structure of this thesis}

The vertebrate sense of smell is a quite remarkable evolutionary story, which is apparent on molecular, anatomical, and functional level. Since the sense of olfaction is invariably linked to the chemical environment, the tetrapod conquest of land and the subsequent radiation into a variety of different habitats have left its mark. Within the framework of my thesis, I have tried to characterize specific aspects of the sense of smell in various vertebrate species with a specific focus on the amphibians. Their evolutionary position at the root of the terrestrial vertebrate lineage, while still maintaining a lifestyle close to the aquatic environment makes them prime organisms to elucidate adaptive processes between two such diverse habitats.

- Chapter 2 contains a published protocol manuscript on dye electroporation and imaging of calcium signaling in the olfactory system of Xenopus laevis tadpoles. The methods have also been used in the other chapters of this thesis (Weiss et al., 2018). 
- Chapter 3 describes the organization of glomerular clusters in the MOB of anuran tadpoles of ecologically diverse species as well as the differences in metamorphotic remodeling between an aquatic and a terrestrial frog. (accepted at Frontiers in Neuroanatomy).

- Chapter $\mathbf{4}$ characterizes the metamorphotic changes of the main olfactory system of Xenopus laevis, with a specific focus on the connection between the periphery and the MOB and cellular and functional features of the 'air-system' vs the 'water-system' (manuscript in preparation)

- Chapter 5 is a comparative study conducted in a jawless and a ray-finned fish species, a neotenic salamander and four ecologically diverse frog species. Tracings of individual RN axons revealed that the unbranched $\mathrm{RN}$ axons hypothesis does not apply to all vertebrates. All examined amphibians (pre- and postmetamorphotic anurans) exhibited axon bifurcations and multi-glomerular innervations (Weiss et al., 2020).

The citation style of the manuscripts was changed to the general style of this thesis and the references are included in the general Reference section at the end of the thesis. Figures, legends, and abbreviations were left unchanged from the published/submitted manuscripts. 


\section{Chapter 2: Dye electroporation and imaging of calcium signaling in Xenopus nervous system}

Published as chapter in Xenopus: Methods in Molecular Biology vol 1865 (2018) edited by Kris Vleminckx and available under https://doi.org/10.1007/978-1-4939-8784-9 15

\section{Authors and affiliations}

Lukas Weiss ${ }^{1 *}$, Thomas Offner ${ }^{12}$, Thomas Hassenklöver ${ }^{1}$ and Ivan Manzini ${ }^{12^{+}}$

${ }^{1}$ Institute of Animal Physiology, Department of Animal Physiology and Molecular Biomedicine, JustusLiebig-University Giessen, 35392 Giessen, Germany

${ }^{2}$ Center for Nanoscale Microscopy and Molecular Physiology of the Brain, University of Göttingen, 37073 Göttingen, Germany

*lukas.weiss@physzool.bio.uni-giessen.de

† ivan.manzini@physzool.bio.uni-giessen.de

\section{Contribution statement}

I wrote the manuscript, conducted the experiments and arranged the Figures for all parts of the manuscript except part 3.5 about functional calcium imaging, which was written and arranged by T.O. All authors were involved in the optimization of the methods and in reviewing and editing the manuscript.

\section{Keywords}

electroporation, neuronal tracing, single cell morphology, fluorescent dyes, calcium imaging, in vivo imaging, Xenopus laevis, olfactory system, olfactory receptor neurons, mitral-tufted cells 


\begin{abstract}
Electroporation is an efficient method of transferring charged macromolecules into living cells in order to study their morphology, function, and connectivity within neuronal networks. Labeling cells with fluorophore-coupled macromolecules can be used to trace projections of whole neuronal ensembles, as well as the fine morphology of single cells. Here we present a protocol to visualize pre- and postsynaptic components of a sensory relay synapse in the brain, using the olfactory system of Xenopus laevis tadpoles as a model. We apply bulk electroporation to trace projections of receptor neurons from the nose to the brain, and single cell electroporation to visualize the morphology of their synaptic target cells, the mitral-tufted cells. Labeling the receptor neurons with a calcium-sensitive dye allows us to record stimulus induced presynaptic input to the dendrites of the postsynaptic cells via functional calcium imaging.
\end{abstract}

\title{
1. Introduction
}

To understand the formation and wiring principles of neuronal networks, it is extremely important to have a robust mode of tracing cells and their processes in living neuronal tissue. Electroporation is an established method that has been used in a variety of cell types and brain regions and has proven to deliver fast results, surpassing some of the drawbacks of other common transfection methods like microinjection, viral transfection, or the creation of transgenic animal lines (Haas et al., 2001). The method relies on the principle of transiently permeabilizing the membrane of cells through the exposure to an electric field. When the trans-membrane voltage exceeds a certain threshold, membrane molecules start to rearrange and form pores that increase the membrane's permeability for different kinds of molecules(Chen et al., 2006). Charged macromolecules like fluorophore-coupled dextrans or plasmid DNA can be transported along the electric field through the membrane pores into the cytoplasm. Upon collapse of the electrical field, the pores re-seal and the cell membrane returns to its prior state. The exact mechanisms leading to pore formation and sealing still remain a topic of discussion (Falk et al., 2007; Ho and Mittal, 1996; Neumann et al., 1999).

The first electroporations performed in neuronal tissue had the goal of genetically transfecting rat (Miyasaka et al., 1999) or chicken embryos (Araki and Nakamura, 1999). Protocols were soon optimized to target neurons in a variety of in and ex utero tissues, as well as postnatal neuronal tissue (De Vry et al., 2010 for an extensive review). Due to its exposed position, the nervous system of Xenopus tadpoles soon proved to be a well-suited model for various approaches of electroporation. By varying the type of electrodes used (plate-like, needle-like, wires, micropipettes), electroporation can be applied to examine the projections of whole nerve fibers (Haas et al., 2002), the structure of microcircuits (Hovis et al., 2010) or even fine morphological details of single cells (Haas et al., 2001, 2002). In addition to the variable cell number labeled using different protocols, the characteristics of variable dyes allows different experimental approaches. Fluorophore-coupled dextrans allow to immediately visualize morphological structures. They are biologically inert and relatively small in size compared to plasmid DNA or fluorescent proteins (Haas et al., 2002). Cell transfection using plasmid 
DNA encoding fluorescent proteins provides the opportunity to monitor the morphological changes of electroporated cells over a longer period of time, and under cell type-specific genetic promoters. For example, in vivo time lapse experiments have been successfully conducted in different parts of the brain of Xenopus laevis tadpoles, the optic tectum (Bestman et al., 2006; Cline and Haas, 2008; Haas et al., 2002; Hewapathirane and Haas, 2008) and the olfactory system (Hassenklöver and Manzini, 2014). Furthermore, electroporation with calcium-sensitive dyes provides the opportunity to examine cellular activity through transient changes in intracellular calcium levels, as demonstrated in Danio rerio (Kassing et al., 2013) and Xenopus laevis (Gliem et al., 2013).

In this chapter, we describe a combination of two electroporation methods applied to study the functional connectivity between axon terminals of olfactory receptor neurons (presynapse) and single mitral-tufted cells (postsynapse) in the olfactory bulb of Xenopus laevis tadpoles. Bulk electroporation using the calcium-sensitive dye Oregon Green BAPTA dextran is used to label the axonal projections originating in the olfactory epithelium of the animals and measure their activity upon odorant stimulation via functional calcium imaging. In addition, single mitral-tufted cells and granule cells are electroporated with fluorophore-coupled dextrans, delivered via micropipette electroporation. The colocalization between presynaptic calcium transients following odorant stimulation and mitral-tufted cell dendritic morphology provides insight into the odorant information conveyed at the respective synaptic connection (Gliem et al., 2013; Hassenklöver and Manzini, 2013).

\section{Materials}

\subsection{Animals}

Xenopus laevis tadpoles of either sex, staged 50 to 55 (staged after Nieuwkoop and Faber, 1994).

\subsection{Solutions and Dyes}

1. Frog saline ringer: $98 \mathrm{mM} \mathrm{NaCl}, 2 \mathrm{mM} \mathrm{KCl}, 1 \mathrm{mM} \mathrm{CaCl} 2,2 \mathrm{mM} \mathrm{MgCl} 2,5 \mathrm{mM}$ glucose, $5 \mathrm{mM} \mathrm{Na}$ pyruvate, $10 \mathrm{mM}$ Hepes, $\mathrm{pH} 7.8$, osmolarity $230 \mathrm{mOsmol} / \mathrm{l}$, stored at $4^{\circ} \mathrm{C}$.

2. Anesthetic: $0.02 \% \mathrm{MS}-222$ (ethyl-3-aminobenzoat methanesulfonate; Sigma Aldrich) in tap water, $\mathrm{pH} 7.8$, stored at $4^{\circ} \mathrm{C}$.

3. Dextran coupled dyes (e.g. 3 mM Alexa 594 dextran, 10000 kDa MW, Life Technologies; 3 mM Oregon Green 488 BAPTA-1 dextran, 10000 kDa MW, Life Technologies). Both dyes were dissolved in frog saline ringer.

4. Odorant stimuli for calcium imaging diluted in frog saline ringer (e.g. $100 \mu \mathrm{M} \mathrm{L}$-histidine, L-lysine, Larginine, L-phenylalanine, L-tryptophan, L-methionine, L-leucine, L-isoleucine, L-valine).

\subsection{Micropipette fabrication}

1. Micropipette puller (e.g. P 1000, Sutter Instruments). 
2. Borosilicate glass capillaries containing an internal filament (outer diameter $1.5 \mathrm{~mm}$, inner diameter $0.86 \mathrm{~mm}$, length $100 \mathrm{~mm}$ ).

\subsection{Electroporation devices and microscopes}

1. Dissection microscope with fluorescent illumination and appropriate filter sets. The objective needs to have a large working distance for tissue preparation.

2. Voltage pulse generator (e.g. ELP-01D, NPI Electronics) connected to two platinum wire electrodes (e.g. diameter $0.2 \mathrm{~mm}$ ). Optimally, a capacitor is connected into the circuit in parallel.

3. Upright microscope with fluorescence detection and appropriate filter sets for single cell electroporation (with water immersion objective with numerical aperture $=1$ and working distance $=$ $2 \mathrm{~mm})$.

4. Single Cell Electroporator (e.g. Axoporator 800A, Axon Instruments/Molecular Devices). The headstage of the Axoporator contains an electrode holder and is equipped with a wire electrode. The electrode is preferably coated with $\mathrm{AgCl}$ and connects to the dye in the micropipette. Additionally, a reference electrode is connected to close the electrical circuit.

5. Micromanipulators (e.g. Scientifica PatchStar). The micromanipulator should be motorized and remotely controllable.

6. Confocal or multiphoton microscope.

\section{Methods}

\subsection{Bulk electroporation of fluorophore-coupled dextrans in the tadpole nose}

1. Prior to electroporation, produce small dye crystals by dissolving the fluorophore-coupled dextrans in frog saline ringer at a concentration of $3 \mathrm{mM}$ and carefully place drops of the solution on a piece of parafilm. Let the dye drops dry at room temperature for at least $30 \mathrm{~min}$ before you use the resulting dehydrated dye crystals for electroporation or store them in a freezer at $-20^{\circ} \mathrm{C}$ (see Note 1). Always keep the dye in the dark during the procedure to prevent photo-bleaching.

2. Select a Xenopus tadpole of desired developmental stage and anesthetize it in a small glass beaker containing $0.02 \% \mathrm{MS}-222$ dissolved in tap water for up to 5 minutes. Test the tadpole's responsiveness to mechanical stimuli in order to confirm proper anesthesia. If not responsive, transfer the tadpole to a wet paper tissue placed in a Petri dish (see Note 2).

3. Center the region of interest (e.g. the nostrils of the tadpole) in the field of view of the dissection microscope (Fig. 1a) and gently dry the moisture using a paper towel to prevent the fluorescent dye from spreading (see Note 3). Apart from the region of interest, the animal should be covered in ringer solution throughout the procedure to keep it moist. 
4. Use fine forceps to pick up a crystal (e.g. Oregon Green BAPTA dextran crystal) and place it in the nasal cavity, where it will dissolve in the residual moisture (white arrowheads in Fig. 1b). Use the fluorescent illumination of the dissection microscope to monitor the confined spread of the fluorophore-coupled dextran in the nasal cavity (see Note 4).

5. Connect two platinum wire electrodes (e.g. diameter $0.2 \mathrm{~mm}$ ) to a voltage pulse generator with a capacitor that is connected in parallel. Insert each electrode into one of the dye filled nasal cavities without injuring the olfactory mucosa (see Note 5, Fig. 1c). Apply 12 pulses ( $25 \mathrm{msec}$ duration at $2 \mathrm{~Hz}$ ) with alternating polarity at a voltage of $20 \mathrm{~V}$ (see Note 6).

6. After removing the electrodes, carefully transfer the tadpole into a beaker with tap water for recovery. The water column in the recovery beaker should not exceed 2 to $3 \mathrm{~cm}$ (see Note 7).
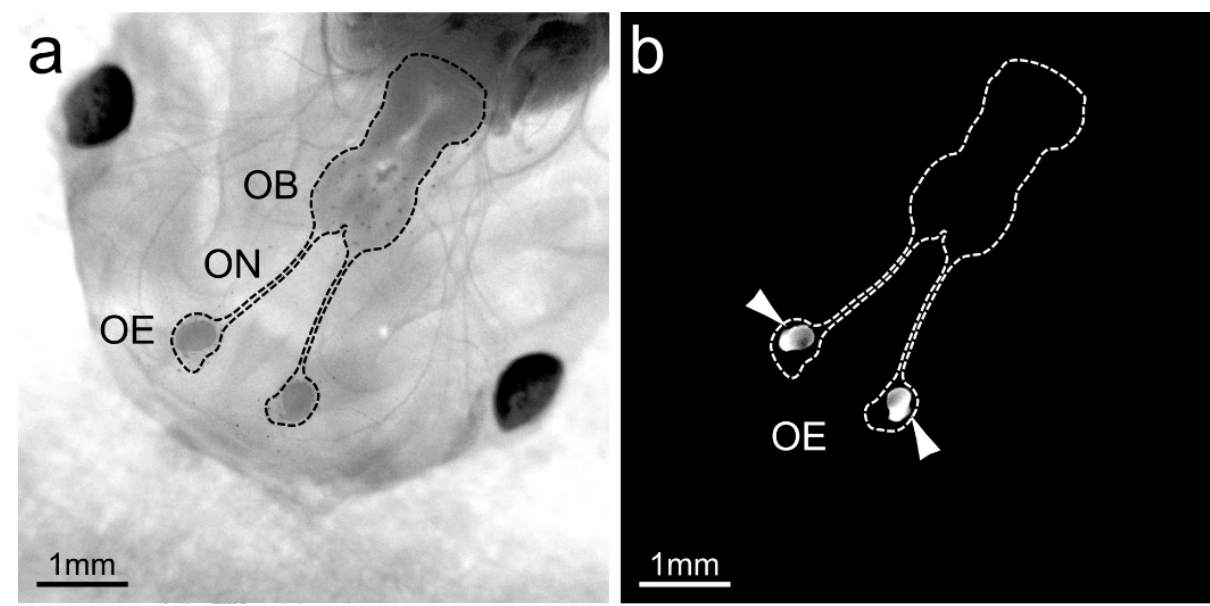

Fig. 1 Bulk electroporation in the nose of Xenopus laevis tadpoles using

fluorophore-coupled dextran crystals.

(a) Overview of the tadpole head under the dissection microscope. The black dotted line highlights the olfactory system and the anterior part of the brain. (b)
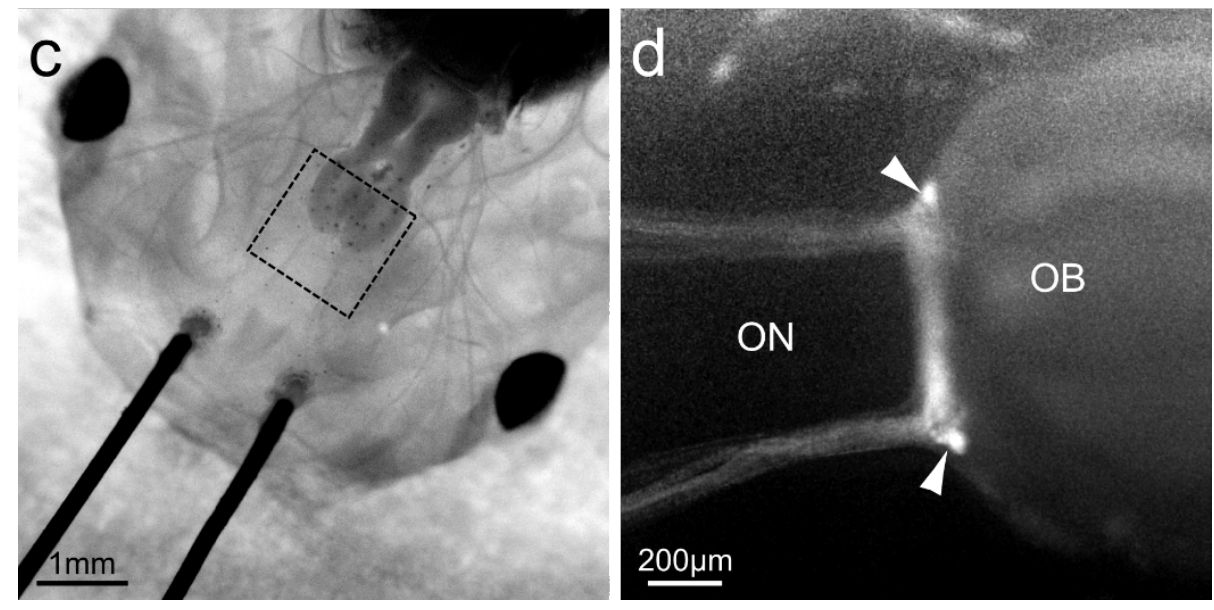
After placing the dye crystals in the nostrils, they dissolve in the nasal moisture (white arrowheads). The image shows the olfactory system and the anterior part of the brain (dotted line) using fluorescent illumination. (c) Two wire electrodes are placed in the nasal cavities for electroporation. The dotted square indicates the section shown in $\mathrm{d}$. (d) The electroporated dye (Oregon Green BAPTA dextran) is transported along the axons of olfactory receptor neurons via the olfactory nerve to the olfactory bulb 24 hours after electroporation. Image was taken using fluorescent illumination. The white arrowheads highlight the axon terminals that are filled with the dye and arranged in characteristic clusters. $O E$ olfactory epithelium, ON olfactory nerve, OB olfactory bulb. 
7. The dextran dye is transported along the nerve fibers, in this case the olfactory nerve, to the axonal terminals located in the olfactory bulb (Fig. 1d).

8. Check for labeled sensory neurons in the nose and olfactory bulb using the fluorescence illumination of the dissection microscope (white arrowheads in Fig. 1d) before continuing with the labeling of single neurons in the brain, e.g. mitral-tufted cells.

\subsection{Pipette fabrication for sparse cell labeling in the brain}

1. Fabricate electroporation micropipettes from borosilicate glass capillaries using a horizontal micropipette puller.

2. Modify the protocol to obtain micropipettes with a small tip opening, long taper and a high pipette resistance of around 15 to $20 \mathrm{M} \Omega$ (see Note 8). The pipette tip opening should be around 1 to $2 \mu \mathrm{m}$ (Bestman et al., 2006) in diameter and the pipette should be rigid enough not to break when penetrating the tissue.

3. Slowly fill the pipettes with 3 to $5 \mu \mathrm{l}$ of the solution (e.g. Alexa 594 dextran) using a microloader pipette tip and make sure to avoid the formation of bubbles within the fluorophore solution, especially in the taper of the micropipette (see Note 9).

4. Mount the micropipette on the holder of the micromanipulator, ensuring that the $\mathrm{AgCl}$ coated silver wire of the headstage of the Axoporator is in contact with the fluorophore solution (see Note 10, Fig. 2).

\subsection{Whole mount preparation}

1. Anesthetize the tadpole in a small glass beaker containing $0.02 \% \mathrm{MS}-222$ as described above (3.1.2).

2. Sacrifice the tadpole by severing the region between brainstem and spinal cord with a scalpel. Subsequently cut a rectangular tissue block out of the upper jaw and palate of the animal containing both nostrils, the olfactory nerves, and the olfactory bulb.

3. Make sure to always keep the tissue block covered with frog saline ringer to keep the tissue from drying out.

4. Carefully pin the tissue block to a silicone filled preparation dish using fine needles with the ventral surface of the block facing up. Remove the soft palatial tissue covering the olfactory bulb with fine forceps. Cut alongside the olfactory bulb on both sides using fine scissors until the point where the olfactory nerve connects to the bulb. Be sure to not damage the olfactory nerves or the brain tissue. Lift the cartilaginous palatial lid covering the olfactory bulb and remove it from the tissue block to fully expose the olfactory bulb. 


\subsection{Electroporation of mitral-tufted cells using fluorophore-coupled dextrans}

1. Transfer the tissue explant to a plastic Petri dish with a small rectangular recess in the middle and mechanically fix it with a grid made of a small platinum frame stringed with nylon threads (see Note 11, Fig. 2). Cover the tissue block with ringer solution.

2. Carefully place the reference electrode connected to the headstage of the Axoporator near the tissue block, and make sure it is in contact with the solution to close the electrical circuit (Fig. 2).

3. Immerse the objective of the upright microscope (see Note 12) into the solution, and position the tissue explant in a way that your region of interest (in this case the mitral cell layer of the olfactory bulb) is centered in the field of view (see Note 13, Fig. 3a).

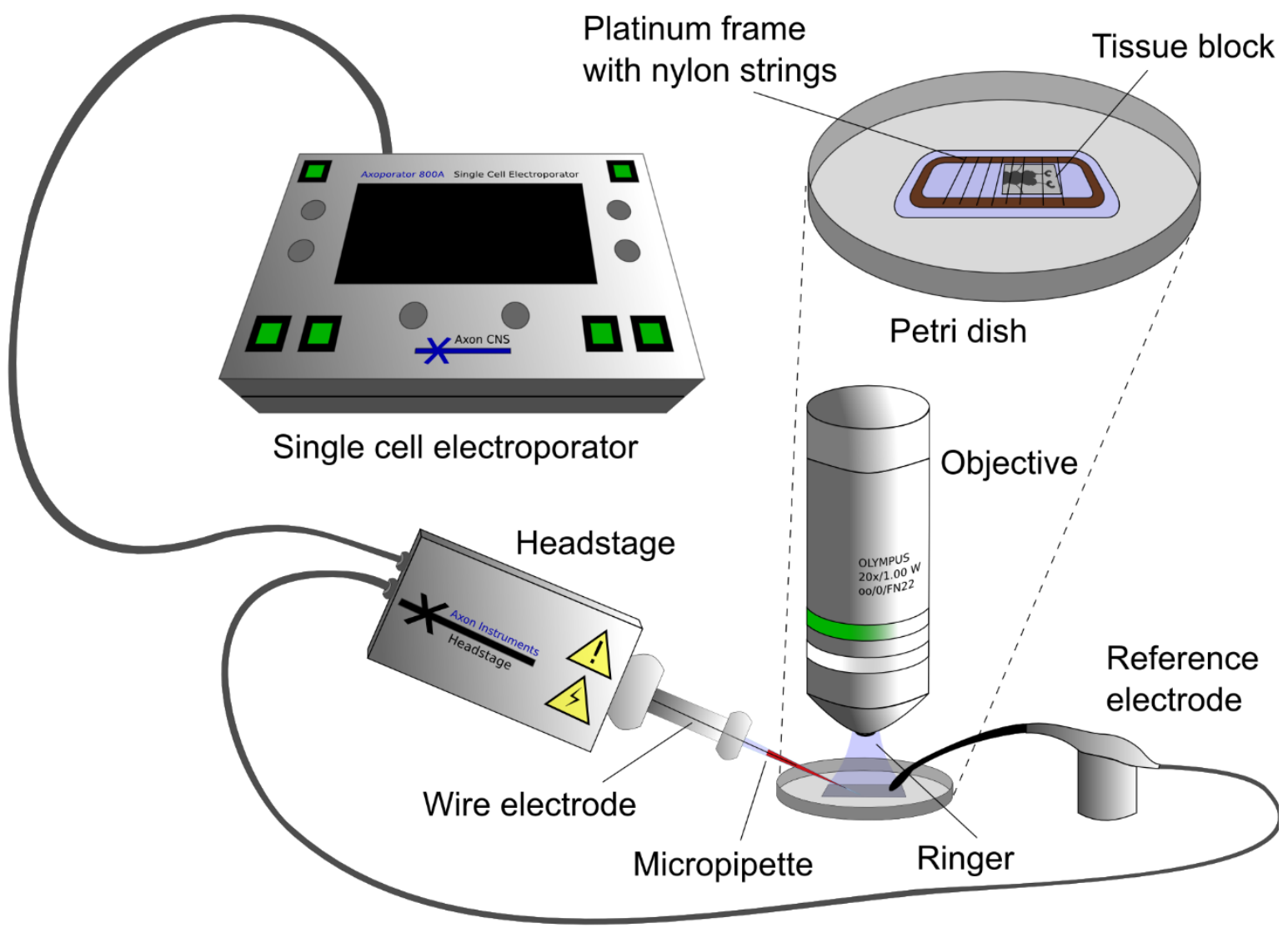

Fig. 2 Schematic of single cell electroporation setup. The single cell electroporator is linked to the headstage and in contact to the fluorophore solution in the micropipette via a wire electrode. The reference electrode is connected to the headstage and immersed in the ringer solution surrounding the tissue block to close the circuit. The tissue block is stabilized on a Petri dish with a platinum frame stringed with nylon threads. 
4. Using the fluorescent illumination of the microscope, you can use the fluorescent signal of the previously electroporated sensory neuron axons (3.1, Fig. 1d) as orientation to help locate your region of interest (see Note 14, green signal in Fig. 3b).
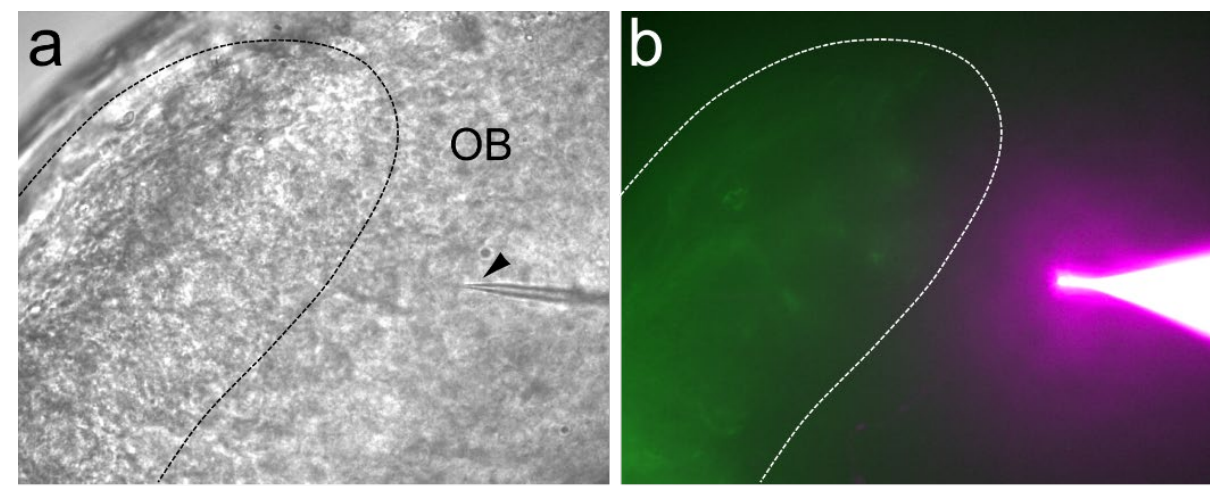

Fig. 3 Single cell electroporation in the olfactory bulb. (a) The micropipette (black arrowhead) is approaching the region of interest, in this case the mitral cell layer of the olfactory bulb. The black dotted line indicates the
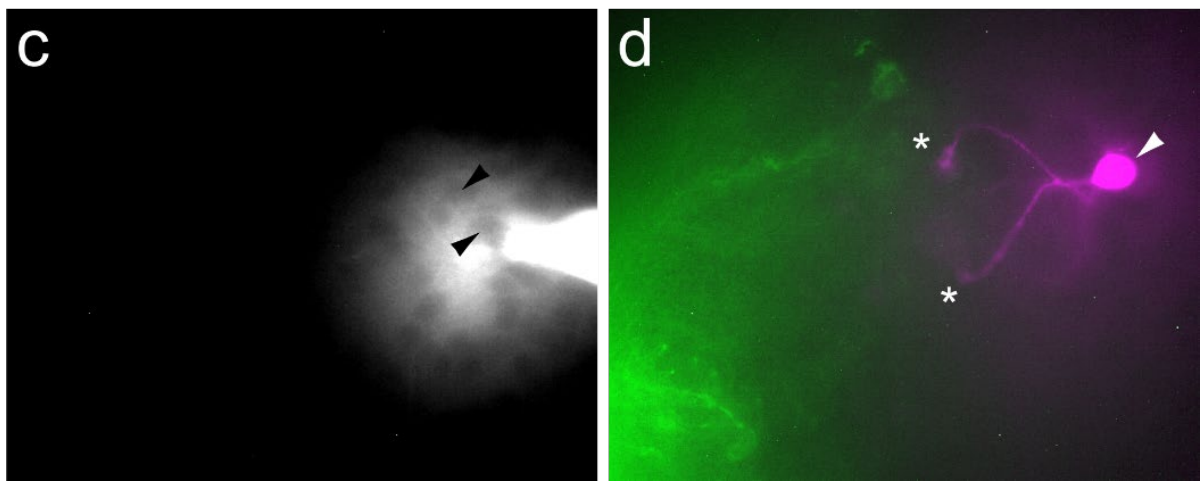
location of sensory neuron axon terminals. (b) The electroporated axon terminals of receptor neurons (Oregon Green BAPTA dextran, green) and the dye filled micropipette (Alexa 594 dextran, magenta) are shown

using fluorescent illumination of a stereomicroscope. (c) After the extrusion of dye into the intercellular space, cells become visible as dark outlines (black arrowhead), which facilitates targeting of a single cell for electroporation. (d) Successful electroporation of a single mitral-tufted cell (magenta) projecting its apical dendrites towards the axon terminals of the receptor neurons (green) via its dendritic tufts (white asterisks). The cell soma is indicated by a white arrowhead. OB olfactory bulb.

5. Remotely control the micromanipulator to position the micropipette in the solution column between objective and tissue (Fig. 2), and carefully approach the pipette tip towards the tissue block along the $\mathrm{z}$-axis (see Note 15, black arrowhead in Fig. 3a).

6. As soon as the pipette touches the surface of the olfactory bulb, activate the virtual approach mode of the micromanipulator to be able to penetrate the tissue along the axis of the micropipette (see Note 10, Note 16).

7. By adjusting and changing the brightness of the light source illuminating the sample you should be able to see some cellular outlines within the tissue that facilitate targeting of a single cell of interest (see Note 13, Fig. 3a, 3c).

8. Once you have approached the micropipette to the proximity of a cell of interest (black arrows in Fig. $3 c$ highlight single cellular outlines), trigger positive square voltage pulses using the Axoporator to 
transfer the dye into the cell (e.g. mitral-tufted cell, Fig. 3d; see Note 17). Use a train of multiple pulses at a voltage of $50 \mathrm{~V}$, pulse length of $300 \mu \mathrm{sec}$ and a frequency of $300 \mathrm{~Hz}$ for $500 \mathrm{msec}$. By applying higher voltage pulses or longer pulse trains of higher frequency, the extent of neuronal labeling (i.e. the number of labeled cells or the intensity of labeling) can be increased (or decreased, if the parameters are lowered).

9. While applying the pulses, monitor the fluorescent dye extrusion and cell staining under appropriate fluorescent illumination (see Note 18, Fig. 3b). If the pipette tip was close enough to a single cell, the fluorescent dye fills the soma (white arrowhead in Fig. $3 \mathrm{~d}$ ) and neurites (white asterisks in Fig. $3 \mathrm{~d}$ ) within seconds after pulse application (see Note 19).

10. Carefully move the micropipette away from the cell and withdraw it out of the tissue using the virtual approach mode of the micromanipulator. The pipette can be used to label further cells at different regions of interest by repeating steps 3.4.6 to 3.4.9.

11. Imaging of the cell morphology and functional calcium imaging can be conducted using a confocal or multiphoton microscope.

\subsection{Functional calcium imaging of odorant-induced activity}

1. Functional calcium imaging is conducted on axon terminals of sensory neurons that have previously been electroporated with the calcium-sensitive dye Oregon Green BAPTA dextran (3.1).We monitor presynaptic activity at the relay synapse to mitral-tufted cells (labeled in 3.4) upon stimulation with odorants. Single amino acids have been found to be potent olfactory stimuli for aquatic animals (Caprio and Byrd, 1984; Manzini and Schild, 2004) and are applied to the nasal cavities.

2. Mount the imaging chamber with the tissue explant containing the olfactory system of the animal on the stage of a confocal or multiphoton microscope.

3. Use a laser wavelength that properly excites the dyes used in the experiments (e.g. $800 \mathrm{~nm}$ wavelength for Oregon Green BAPTA dextran and Alexa 594 dextran) and appropriate emission filters.

4. To visualize the morphology of the electroporated cells, acquire a virtual image stack including the cell and all its processes (Fig. 4a presents a maximum intensity projection of a virtual image stack of mitral-tufted cells).

5. For odorant perfusion, use a gravity feed perfusion system made of plastic tubing.

6. Install the funnel for odorant applications and direct it towards the olfactory epithelium. In order to create continuous and stable flow directed to the olfactory epithelium, position a suction syringe driven by a peristaltic pump caudally to the tissue block into the recording chamber. Suction speed of the pump needs to be adjusted until continuous flow of bath solution is achieved.

7. Direct the focus of the microscope to your region of interest in the bulb via bright field illumination. Then use multiphoton excitation to screen for the fluorescent signal emitted by the electroporated 
axons and postsynaptic cells. Focus on the substructures of interest (e.g. the dendritic tufts of the electroporated mitral-tufted cell, Fig. 4b).

8. Acquire recordings of single or multiple imaging planes of the desired region of interest over time.

9. Increases in intracellular calcium concentration upon odorant stimulation, e.g. single L-amino acids or mixtures at $100 \mu \mathrm{M}$ concentration can be recorded by monitoring temporal changes of fluorescence of the calcium-sensitive dye. Multiple measurements of odorant applications ensure reproducibility of the signal. Leave at least 2 min intervals between measurements to avoid olfactory receptor neuron desensitization.

10. Responsive axon terminals in the image can be further analyzed by normalizing the fluorescence changes over time (Fig. 4c). Colocalization between these responsive terminals and mitral-tufted cell dendrites gives information about odor transmission across this synapse.

11. Further insight into the neuronal network can be obtained by applying electroporation with multiple dyes (Fig. 5a) or targeting different cell types (Fig. 5b mitral-tufted cell, Fig. $5 c$ granule cell).
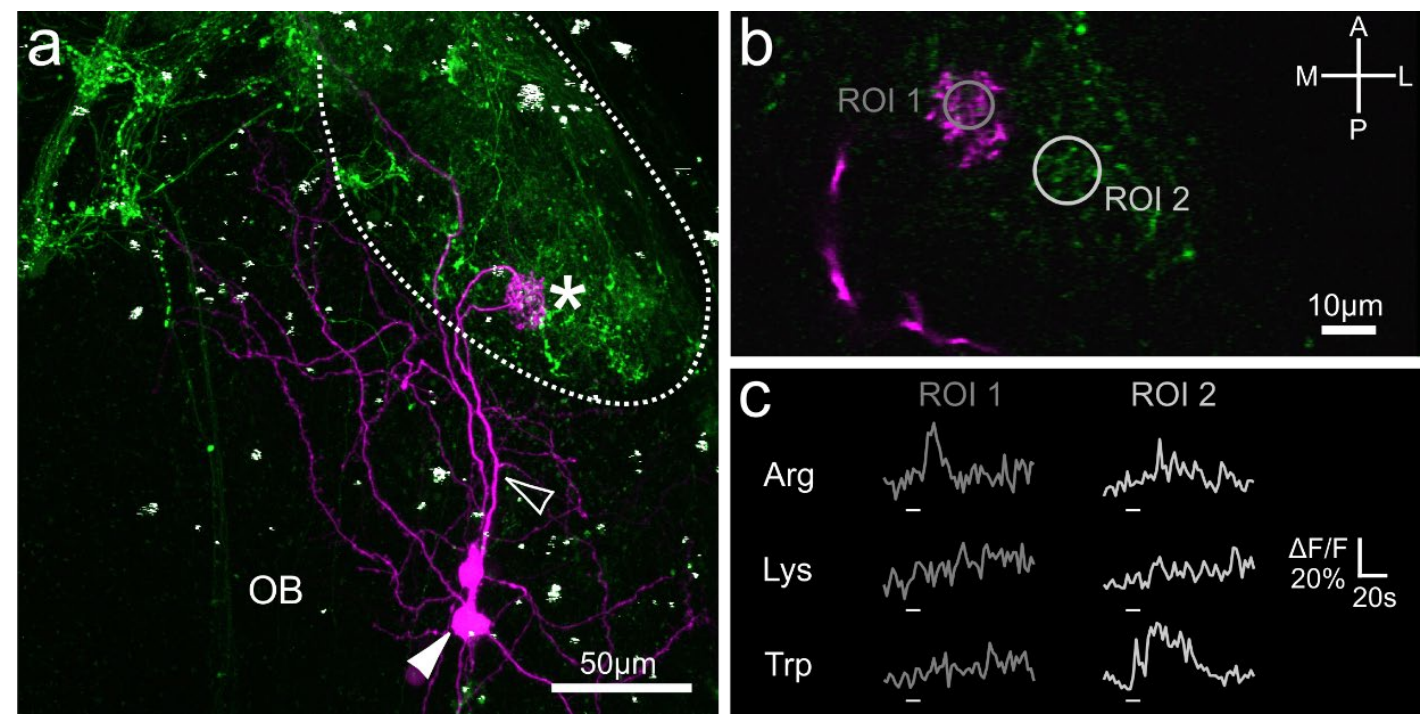

Fig. 4 Functional measurements of presynaptic input on mitral-tufted cells imaged with a multiphoton microscope. (a) Sparse cell labeling of postsynaptic cells (magenta) in the ventral olfactory bulb of Xenopus laevis tadpoles. Olfactory receptor neurons and their axonal projections in the olfactory bulb (green) were previously labeled with dextran-coupled calcium indicator by bulk electroporation into the nostrils. (b) Single imaging plane including the apical tuft of the mitral-tufted cell (magenta) and axonal fibers loaded with Oregon Green BAPTA dextran (green). Odorant induced calcium-dependent changes in fluorescence of the two different regions of interest (ROI 1 in colocalization with the dendritic tuft and ROI 2 in a not connected structure) are shown in c. (c) The normalized fluorescence time courses ( $\triangle \mathrm{F} / \mathrm{F}$; for 90 seconds) of the calcium indicator signal upon 10 seconds of odorant application (lower white bars) are depicted for the respective ROIs. Individual L-amino acids were used as odor stimuli and induce different response profiles in the respective ROIs. ROI1 shows a transient increase in fluorescence upon stimulation with arginine, but not to lysine or tryptophan. ROI2 is responsive to tryptophan, but not to the stimulation with arginine or lysine. OB olfactory bulb, Arg L-arginine, Lys L-lysine, Trp L-tryptophan. 


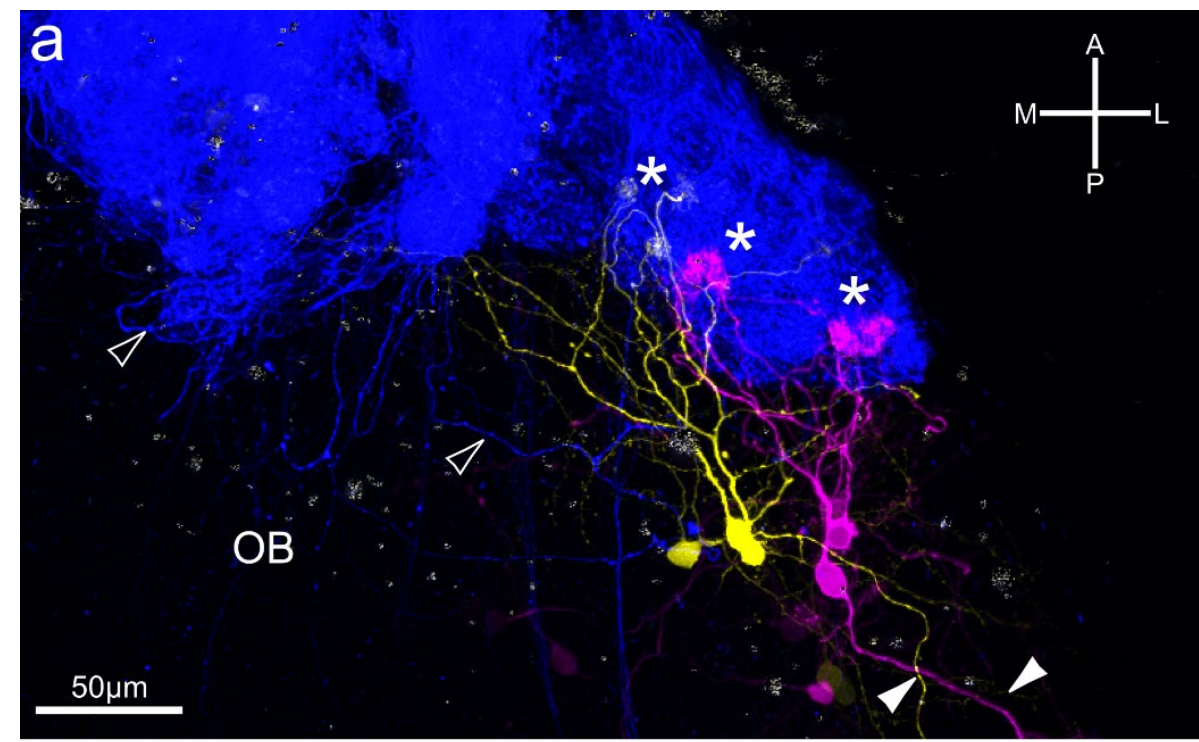

Fig. 5 Examples of successfully labeled cells via electroporation imaged with a multiphoton microscope. The olfactory receptor neuron terminals are electroporated using Cascade blue dextran (blue). (a) Single receptor neuron axons are indicated by empty arrowheads. Multiple mitral-tufted cells were electroporated using two
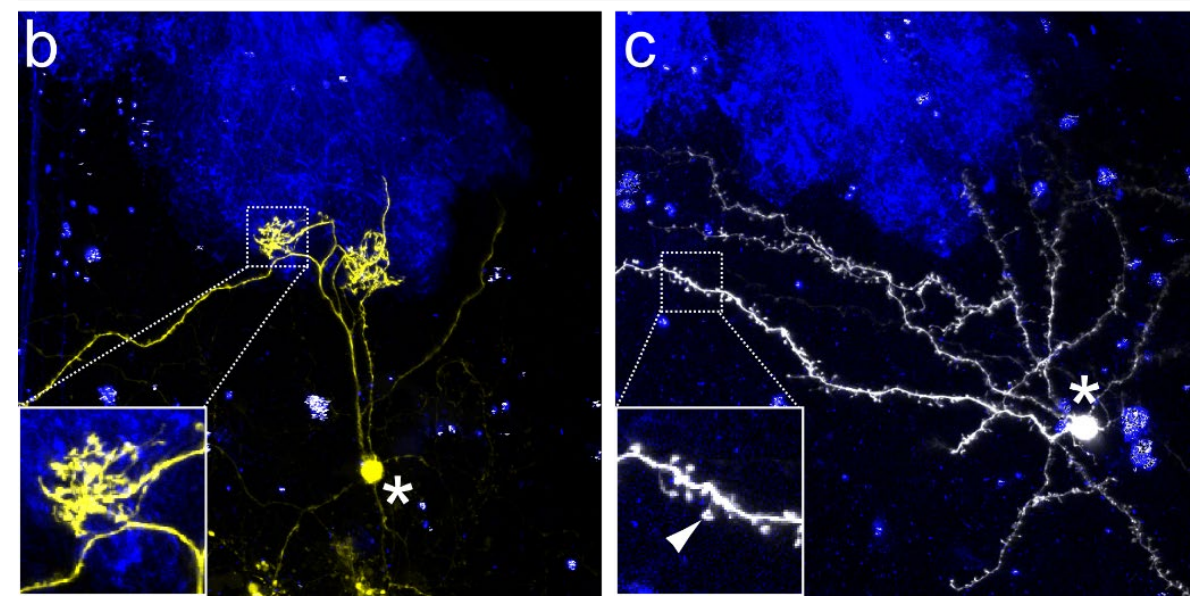
different fluorescent dyes (yellow: Alexa 488 dextran, magenta: Alexa 594 dextran). Their dendritic tufts connect to the receptor neuron terminals (white asterisks). Mitral-tufted cells project their axons towards higher brain centers (filled arrowheads). (b) Example of a single electroporated mitral-tufted cell. One dendritic tuft is displayed in a higher magnification in the inset. The white asterisk indicates the cell soma. (c) A single granule cell is labeled. Granule cells are characterized by long branches that extend over the entire olfactory bulb bearing fine spines on their dendrites (arrowhead in the inset). The cell soma is indicated by an asterisk. All pictures are shown as maximum intensity projections of virtual image stacks acquired with a multiphoton microscope. A anterior, L lateral, P posterior, M medial, OB olfactory bulb.

\section{Notes}

1. Drops of the fluorophore-coupled dextrans should be about $1 \mu \mathrm{l}$. The amount of one dye crystal should suffice to fill up the entire nasal cavity once it is dissolved in the nasal moisture (Fig. 1b)

2. The time required to fully anesthetize the animal varies between animals of different stages. All following references to animal stages are referenced to Nieuwkoop and Faber (1994).

3. When applying bulk electroporation in the nose of postmetamorphotic froglets (stage $66+$ ), it is important to make sure, that the dye enters all nasal cavities. Because of the more segregated, bony 
structure and increased size of the nose in higher staged animals, the dye may not diffuse into the deeper parts of the nasal cavities.

4. If desired, the two nostrils can be electroporated with different fluorophores to label axonal projections from both sides separately. In that case, it is crucial to monitor the dye spread to ensure that the fluorophores do not mix in the moisture of the nostrils.

5. Make sure that the two electrodes are in contact with the fluid in the nasal cavities, otherwise you will not obtain a proper staining of the neurons. If the electrodes are in direct contact with the epithelial tissue in the nose, neurons might be damaged.

6. By increasing the voltage or the number of pulses applied, you can increase the amount of receptor neurons electroporated. Nevertheless, the voltage range is limited, with too low voltages failing to electroporate cells, and too high voltages resulting in cellular damage and even cell death. The ideal voltage should be tested for the electrodes used for the electroporation. The voltage proven to be most effective using our setup ranged from 20 to $30 \mathrm{~V}$ for 12 pulses at a frequency of $2 \mathrm{~Hz}$. The use of a capacitor connected to the electroporation circuit increases successful labeling of cells as voltage pulses show an exponential decay due to capacitance discharge(Haas et al., 2001).

7. To improve recovery of the animals, lower the water level and/or pump oxygen enriched water through their gills with a Pasteur pipette. The bulk electroporation procedure and handling of anesthetized animals should not take longer than 5 minutes.

8. The fabrication of the micropipette might vary according to the experimental question and available equipment. Generally, a small tip opening ( 1 to $2 \mu \mathrm{m}$ ) and a long taper are desirable to confine labeling to a small number of cells or a single cell. If the pipette taper is too long and fragile, it might break when penetrating the tissue. In case a larger number of cells needs to be labeled (Fig. 5a), the tip opening can also be bigger. The right size of the tip opening and taper length can be modified by changing the heat and pulling velocity parameters of the puller (Bestman et al., 2006).

9. If air bubbles form, remove them by flicking the finger against the pipette shaft. In case this does not work, use a new pipette as the bubble can affect the electroporation protocol.

10. Optimally, the micromanipulator should be controllable along a 'virtual approach' axis in addition to the $x-, y$ - and $z$ - axes. This enables the diagonal approach to the sample, along the axis of the pipette and thus facilitates precise placement of the pipette and cellular targeting. Move the pipette along this fourth axis to approach single cells as well as when retracting the micropipette from the tissue.

11. It is essential to fix the tissue block to prevent it from moving while penetrating with the micropipette. The region of interest should optimally be placed between two strings of the grid.

12. Use an upright fluorescent microscope with a large working distance objective to have enough space for the micropipette.

13. With increasing thickness of the tissue (due to higher staged animals for example), the bright field illumination needs to be increased in order to see cellular outlines in the region of interest (Fig. 3a). 
14. Switching between bright field (Fig. 3a) and fluorescent illumination (Fig. 3b), using the previously labeled receptor neurons as a guide (green, Fig. 3b), is the best mode of orientation to find mitraltufted cells in the region of interest.

15. Use the fluorescence filter of the microscope to monitor the pipette tip closely. Always keeping the pipette tip in focus while approaching the tissue and the cells of interest helps to prevent breaking the pipette at the objective or inserting it too far into the tissue block.

16. Before approaching the target cell layer, make sure your bath electrode is in contact to the solution and that there is no outflow of solution from the pipette tip. To fully eliminate the outflow, change the offset voltage on the Axoporator stepwise. If the pipette tip opening is too big, bath solution might be streaming into the tip due to the capillary effect.

17. Monitor the resistance or current flow on the Axoporator closely to estimate whether the pipette is approaching cells, or whether it is clogged. Resistances between 20 to $80 \mathrm{M} \Omega$ are generally a good range for electroporating; if resistances increase drastically above $100 \mathrm{M} \Omega$, the pipette might be clogged, and electroporation might not work properly. If very little dye is extruded from the pipette tip because of an elevated resistance, it usually helps to either increase the voltage to 60 to $70 \mathrm{~V}$ or increase the offset voltage to 0.5 to $2 \mathrm{~V}$. If that does not solve the problem, retract the pipette from the tissue and penetrate a different region while monitoring changes in the pipette resistance. If the pipette remains clogged, it is advisable to exchange the pipette.

18. There are multiple reasons for lack of dye extrusion from the pipette tip. Double check the reference electrode and the offset voltage that can be adjusted on the Axoporator as well as the resistance (compare Notes 16 and 17). If dye is leaving the pipette tip, but no cell is labeled, you should try to move the pipette tip to a different position within the tissue. If dye is extruded into the intercellular space, you might be able to see black outlines of cells nearby (black arrowheads in Fig. $3 c)$. These outlines make it much easier to orientate and point the pipette tip to a single cell soma. The dye in the interstitial area usually leaves no to very little background staining when imaged in a confocal or multiphoton microscope.

19. Electroporated and fluorescently labeled cells often show signs of cell death after the electroporation e.g. decomposition of their appendages. This cell death can be seen in the form of little fluorescent droplets in the tissue. If a cell is electroporated correctly, but the fluorescent signal is weak, the cell can also be electroporated again by repositioning the pipette. 


\section{Chapter 3: Conservation of glomerular organization in the main olfactory bulb of anuran larvae}

Manuscript accepted at Frontiers in Neuroanatomy as Brief Research Report. doi: $\underline{10.3389 / \text { fnana.2020.00044 }}$

\section{Authors and affiliations}

Lukas Weiss ${ }^{1,4,}{ }^{*}$, Lucas D. Jungblut ${ }^{2}$, Andrea G. Pozzi ${ }^{2}$, Lauren A. O'Connell ${ }^{3}$, Thomas Hassenklöver ${ }^{1}$ and Ivan Manzini ${ }^{1}$

1 Department of Animal Physiology and Molecular Biomedicine, Justus-Liebig-University Giessen, 35392 Giessen, Germany

2 Departamento de Biodiversidad y Biología Experimental, IBBEA-CONICET, Universidad de Buenos Aires, C1428EGA Buenos Aires, Argentina

3 Department of Biology, Stanford University, 94305 Stanford, California, USA

4 Lead Contact

*Correspondence: lukas.weiss@physzool.bio.uni-giessen.de (L.W.)

\section{Contribution statement}

I conducted all experiments and analyses, arranged the figures and wrote the first draft of the manuscript. I conceptualized the study together with T.H. and I.M. L.D.J, A.G.P and L.A.O provided labspace, animals and materials. All authors were involved in reviewing and editing the manuscript.

\section{Keywords}

amphibians, anura, olfaction, glomeruli, olfactory bulb, evolution 


\begin{abstract}
The glomerular array in the olfactory bulb of many vertebrates is segregated into molecularly and anatomically distinct clusters linked to different olfactory functions. In anurans, glomerular clustering is so far only described in Xenopus laevis. We traced olfactory projections to the bulb in tadpoles belonging to six distantly related anuran species in four families (Pipidae, Hylidae, Bufonidae, Dendrobatidae) and found that glomerular clustering is remarkably conserved. The general bauplan consists of four unequally sized glomerular clusters with minor inter-species variation. During metamorphosis, the olfactory system undergoes extensive remodeling. Tracings in metamorphotic and juvenile Dendrobates tinctorius and Xenopus tropicalis suggest a higher degree of variation in glomerular organization after metamorphosis is complete. Our study highlights, that the anatomical organization of glomeruli in the main olfactory bulb is highly conserved, despite an extensive ecomorphological diversification among anuran tadpoles, which suggests underlying developmental constraints.
\end{abstract}

\title{
Introduction
}

As in most tetrapods, the olfactory periphery of anuran amphibians is segregated into a main olfactory epithelium (MOE) and a vomeronasal organ (VNO; Eisthen, 1997; Reiss and Eisthen, 2008). In addition, several smaller olfactory surfaces have been characterized as specific adaptations to either aquatic olfaction (recessus olfactorius and middle chamber epithelium of the pipid frogs; Helling, 1938; Jungblut et al., 2017; Nowack et al., 2013), aerial olfaction (eminentia olfactoria; Helling, 1938) or possible feeding mechanisms (buccal exposed epithelium; Jungblut et al., 2017). Odorant detection in vertebrates relies on ciliated or microvillous olfactory receptor neurons (ORNs) expressing a single allele belonging to one of several multigene families coding for G-protein coupled olfactory receptors (Buck and Axel, 1991; Dulac and Axel, 1995; Greer et al., 2016; Herrada and Dulac, 1997; Liberles and Buck, 2006; Rivière et al., 2009). In amphibians, each ORN residing in the MOE projects its axon towards one or multiple spheroid neuropil structures (glomeruli) in the main olfactory bulb (MOB) (Hassenklöver and Manzini, 2013; Weiss et al., 2020), where synapses with postsynaptic projection neurons are formed.

The glomerular array of many vertebrates is organized in anatomical and functional clusters (Baier and Korsching, 1994; Braubach et al., 2012; Frontini et al., 2003; Gaudin and Gascuel, 2005). A detailed account of glomerular organization in anurans is available only from the fully aquatic Xenopus laevis (Manzini and Schild, 2010). X. laevis tadpoles have at least two separate odor processing streams from the MOE to spatially segregated glomerular clusters in the MOB. These streams rely on different ORN types, second messenger cascades and odorant receptor types (Gliem et al., 2013; Manzini et al., 2002). Comparative studies with other anuran species are necessary to understand the relevance of this organization. 
The lifecycle of most anurans contains an aquatic larva transforming into an adult frog that dwells on trees, in the water or underground (Duellman and Trueb, 1994; Wells, 2007). However, tadpoles have also diversified and adapted to a variety of aquatic and semiaquatic habitats (Altig and McDiarmid, 1999; Roelants et al., 2011). One distinctive feature among tadpole morphotypes is the oral apparatus and in particular the presence or absence of keratinized mouthparts, which has an impact on the trophic niche occupied by the animals (Altig and McDiarmid, 1999; Orton, 1953). Integrating morphological traits and habitat choice led to the categorization of tadpoles into ecomorphological guilds (Altig and Johnston, 1989). However, little is known about how sensory system anatomy and function are adapted to the specific demands presented by various habitats.
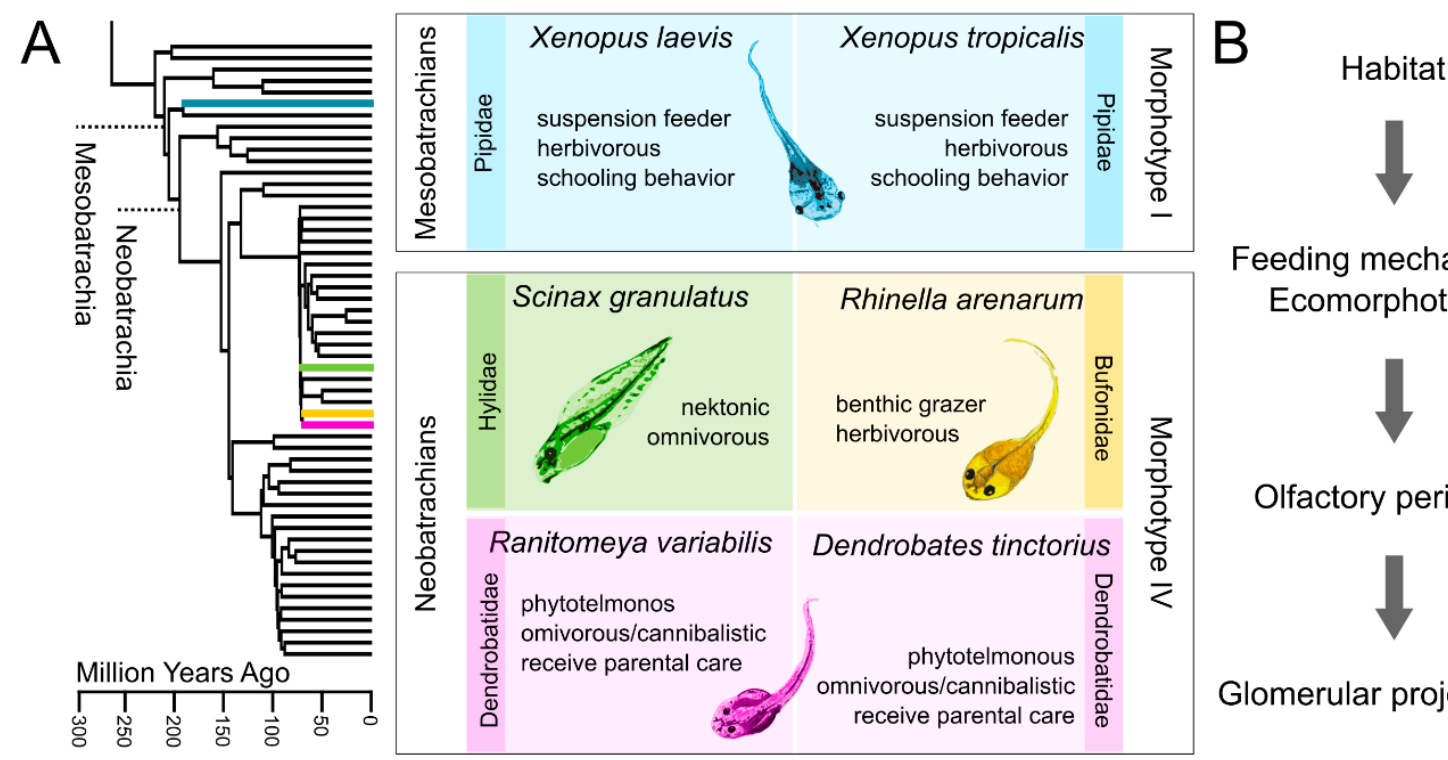

Feeding mechanisms/ Ecomorphotypes

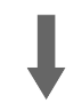

Olfactory periphery<smiles>C1C2CC1C2</smiles>

Glomerular projections?

Figure 1 Diversity of anuran tadpoles used in this study. (A) The phylogenetic tree on the left is pruned from Pyron (2014), which originally includes 3309 species. The four family to which the six examined species belong, are highlighted. The middle panel describes the six species based on phylogeny, ecology, and morphology. Both Xenopus species belong to earlier diverging Mesobatrachians, lack keratinized mouthparts (morphotype I) and are thus obligate suspension feeders. The four neobatrachian species can all be classified as morphotype IV. Their developed mouthparts enable them to scrape off food from the substrate. The two dendrobatid species both receive parental care and mostly live in pools in leaf axils or bromeliads. Morphotype distinction follows (Orton, 1953) and ecomorphotypic categorizations are based on (Altig and Johnston, 1989). (B) Experimental concept of this study. We tried to examine whether the glomerular organization in the MOB of tadpoles is influenced by the respective habitat or feeding mechanisms in the distantly related species.

We analyzed and compared the glomerular organization in the $\mathrm{MOB}$ of larval anurans belonging to six different species of four families (species overview in Figure 1). Glomeruli of all examined tadpoles showed an anatomical segregation into distinct glomerular clusters. The conserved olfactory bulb architecture between the two members of early diverging pipid frogs and later diverging neobatrachian frogs suggests an evolutionary constraint in the glomerular configuration in anuran 
tadpoles. Furthermore, we provide an outlook, that the organization of glomerular clusters in postmetamorphotic frogs might be more variable than in tadpoles.

\section{Materials and Methods}

\section{Experimental animals}

Wild type and albino tadpoles of Xenopus laevis and larvae of wild type Xenopus tropicalis were bred and reared at the Institute of Animal Physiology, Justus-Liebig-University Giessen and kept in water tanks at a water temperature of $20^{\circ} \mathrm{C}$ and $25^{\circ} \mathrm{C}$ respectively. Tadpoles were fed with algae until the end of metamorphosis. Ranitomeya variabilis larvae and larvae and juveniles of Dendrobates tinctorius were bred and reared in the Department of Biology at Stanford University, Palo Alto, CA, USA. Individual tadpoles were kept separately after hatching at a water temperature of $25^{\circ} \mathrm{C}$ and fed with brine shrimp flakes and tadpole pellets, juveniles were kept in terraria and fed with flies. Rhinella arenarum tadpoles were obtained by in vitro fertilization from a colony at the Faculdad de Ciencias Exactas y Naturales of the University of Buenos Aires. Larvae of Scinax granulatus were collected from temporary ponds in the surroundings of the Campus of the University of Buenos Aires. All larvae were kept in tanks of dechlorinated water at $22^{\circ} \mathrm{C}$ and fed with chard leaves. The sex of the developing gonad in the tadpoles and juveniles was not determined in any of the species.

An overview of the quantitatively analyzed samples of premetamorphotic larvae (Figure 2 ) is shown in Table 1. Experiments in higher staged tadpoles and juveniles were conducted for Dendrobates tinctorius (one animal stage 41 , and two juveniles stage 45 after Gosner, 1960) and Xenopus tropicalis (seven metamorphotic animals staged 55, 56, 58, 58, 61, 63 and 65 after Nieuwkoop and Faber, 1994).

$\begin{array}{llll}\text { Species } & \text { Animals } & \text { Olfactory bulb hemispheres } & \text { Stages } \\ \text { Xenopus laevis } & 6 & 11 & 49-54 \mathrm{NF} \\ \text { Xenopus tropicalis } & 5 & 9 & 51-52 \mathrm{NF} \\ \text { Scinax granulatus } & 3 & 5 & 31-33 \mathrm{G} \\ \text { Rhinella arenarum } & 6 & 10 & 29-34 \mathrm{G} \\ \text { Ranitomeya variabilis } & 2 & 4 & 27 \mathrm{G} \\ \text { Dendrobates tinctorius } & 5 & 9 & 25-27 \mathrm{G}\end{array}$

Table 1: Overview of premetamorphotic animals examined in this study. Stages were determined according to Nieuwkoop and Faber (1994) (NF) for the genus Xenopus and using the Gosner (1960) table (G) for all other species. 


\section{Tracing of olfactory projections}

Animals were anaesthetized in 0.02\% MS-222 (ethyl 3-aminobenzoate methanesulfonate; SigmaAldrich) for approx. 5 minutes and placed on a wet paper towel under a stereoscope with fluorescent illumination. Olfactory projections from the nasal cavity to the MOB were labelled using Wheat-Germ agglutinin (WGA) -coupled fluorophores (10 $\mathrm{gg} / \mu \mathrm{l}$, WGA-Alexa Fluor 594/488, Thermo Fisher) dissolved in frog Ringer (in mM: $98 \mathrm{NaCl}, 2 \mathrm{KCl}, 1 \mathrm{CaCl}_{2}, 2 \mathrm{MgCl}_{2}, 5$ glucose, 5 Na-pyruvate, 10 Hepes, pH 7.8). The WGA-fluorophore solution (3-5 $\mu$ l) was pipetted into the nostrils using a microloader and left to be taken up by ORNs for 10 minutes, while the animal was kept moist. Subsequently, the remaining solution was washed off and the animal transferred to a glass beaker to recover. After at least 24 hours the animals were again anesthetized and killed by severing the spinal cord at the level of the brainstem. The olfactory nerves were transected close to the nose and the brain containing the olfactory nerves and the bulbs were dissected out of the tissue. Samples were either scanned immediately without fixation or fixed in 4\% PFA in PBS for one hour and imaged at a later timepoint.

\section{Imaging and image processing}

The olfactory bulbs were imaged with the ventral surface up and fixed with nylon stringed frames in a recording chamber. Image stacks were recorded at a z-resolution of $3 \mu \mathrm{m}$ using multiphoton microscopy (upright Nikon A1R-MP and upright Leica SP5 multiphoton microscopes; excitation wavelength $780 \mathrm{~nm}$ ) and processed in ImageJ (Schindelin et al., 2012). Since only one fluorophore was introduced per sample, we recorded pigmentation-derived auto-fluorescence by simultaneously recording with detectors of different emission-wavelength. In animal species with high pigmentation, we mathematically subtracted this auto-fluorescent signal from the images using the Image calculator function in ImageJ. Brightness and contrast were adjusted, and median filters were applied where necessary. Images showing both olfactory bulbs were stitched together using the stitching algorithm developed by Preibisch et al. (2009). All images presented are rendered in 3D using the 3D viewer plugin implemented in ImageJ.

\section{Volume measurements and statistics}

Glomerular clusters were manually identified, contoured on various z-planes of the image stacks and interpolated using the Segmentation Editor in ImageJ. The contours of a cluster were drawn according to the following criteria: clusters are spatially separated and connect to the olfactory nerve via axon fascicles. No fascicles between clusters were observed, thus resulting in a gap between two clusters. The segments labelled and measured did not include the nerve fibers projecting to these clusters. The volumes of the labelled clusters were analyzed using Python and their relative share of the total glomerular volume is presented in percentages. Averaged data are presented as mean \pm standard deviation. For statistical analysis, the relative volumes of olfactory projections in each bulb hemisphere were considered as independent samples. Statistical significance was assessed using a One-Way ANOVA separately for each of the four clusters followed by Student t-tests for multiple comparison. To control familywise error, a Holm-Bonferroni correction was applied. 


\section{Results}

We first compared larval glomerular organization by tracing the projections of ORNs from the MOE to the MOB (Figure 2). Wheat-Germ-Agglutinin (WGA) tracings of the most distal part of the left olfactory nerve $(\mathrm{ON})$ and the glomeruli for each species are shown. Glomeruli can be discerned as spheroid accumulations of WGA (white arrowheads, Figure 2A). The glomerular array in all species is segregated into four unequally sized clusters (white dotted lines, Figure 2B), best described by their location as lateral (LC), intermediate (IC), medial (MC) and dorso-medial cluster (DC) (Gaudin and Gascuel, 2005; Manzini et al., 2007; Nezlin et al., 2003). This organization is conserved between all species with only minor differences. The LC in Rhinella arenarum tadpoles shows a clear bipartition into a dorsal and ventral lobe (white asterisk, bufonid tracings, Figure $2 \mathrm{~A}$ ). This bipartition is also present in the two Xenopus species, but not equally apparent as in $R$. arenarum. In addition to the four bigger clusters, a few ventro-posterior glomeruli could be observed in all species but were not clearly identifiable in all samples (empty arrowheads, Figure 2A) (Brinkmann and Schild, 2016). Glomeruli in the DC were more clearly discernible in the Neobatrachians compared to Xenopus.

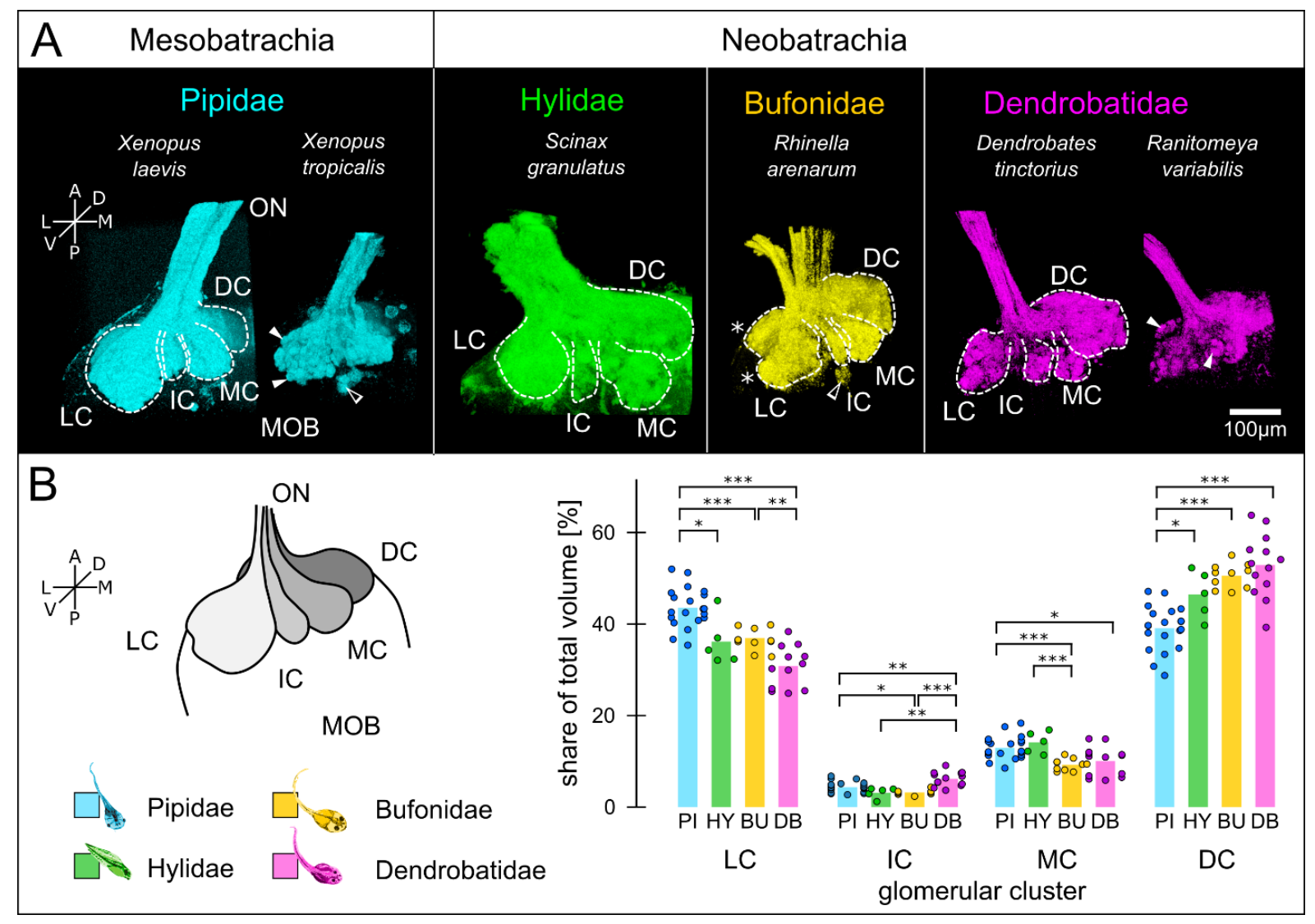

Figure 2 Glomerular clustering in the MOB is conserved among anuran tadpoles. (A) Glomeruli in the MOB of all species can be segregated into three ventrally (LC, IC, MC) and one dorso-medially located clusters (DC). White dotted lines - cluster outlines, filled arrowheads - glomeruli, empty arrowhead - small glomerular cluster, asterisks - ventral and dorsal lobes of the LC. (B) The relative volume of the clusters (schematically shown on the left) varies between the four families. Volumes of the clusters relative to the total glomerular volume for each 
family are shown. Each dot represents one $\mathrm{MOB}$ hemisphere and species of the same family are grouped together. Significance levels: ${ }^{* * *} \mathrm{p}<0.001,{ }^{* *} \mathrm{p}<0.01,{ }^{*} \mathrm{p}<0.05$. A anterior, $\mathrm{P}$ posterior, L lateral, M medial, D dorsal, $V$ ventral, ON olfactory nerve, MOB main olfactory bulb, LC lateral cluster, IC intermediate cluster, MC medial cluster, DC dorso-medial cluster, PI Pipidae, HY Hylidae, BU Bufonidae, DB Dendrobatidae.

We then measured the percentual share of the clusters relative to the total glomerular volume (schematically shown in Figure 2B). Data from species belonging to the same family were pooled together. Across all species, the $L C$ and the $D C$ were most prominent with a combined relative volume of approx. $80 \%$. In pipid tadpoles ( $\mathrm{n}=20$ olfactory bulbs / 11 animals; cyan in Figure $2 \mathrm{~B}$ ), the $\mathrm{LC}$ is slightly bigger than the DC, with $43.6 \pm 4.3 \%$ and $39.1 \pm 4.9 \%$ of the total volume, respectively. Contrastingly, in tadpoles of the other families (Hylidae: $n=5 / 3$, green; Bufonidae: $n=10 / 6$, yellow; Dendrobatidae: $\mathrm{n}=13 / 7$, magenta), the $\mathrm{DC}$ is bigger than the $\mathrm{LC}$, with $46.5 \pm 5.2 \%$ compared to $36.2 \pm 5.4 \%$ in Hylidae, $50.6 \pm 2.7 \%$ to $37 \pm 2.1 \%$ in Bufonidae and $52.9 \pm 6.9 \%$ to $30.8 \pm 4.4 \%$ in Dendrobatidae. The percentual share of the $L C$ in the Pipidae is significantly higher than in the other families (Figure 2B, left) and the $D C$ is significantly smaller (Figure $2 B$, right). The IC and the $M C$ in all species are smaller than the other two clusters with approx. 5 and $10 \%$ of the total glomerular volume, respectively. The IC is biggest in the dendrobatid tadpoles $(6.2 \pm 1.6 \%)$ and significantly bigger than the IC in the other families (Pipidae: $4.4 \pm 1.2 \%$; Hylidae $3.2 \pm 1.2$, Bufonidae: $3.2 \pm 0.5)$. The $M C$ is smallest in the Bufonidae $(9.3 \pm 1.3 \%$; Figure $2 \mathrm{~B}$ ) and biggest in hylid tadpoles $(14.2 \pm 2.4 \%)$. The $\mathrm{MC}$ of the dendrobatid tadpoles (10.1 \pm $3.2 \%)$ is also smaller than in the pipids $(12.9 \pm 2.5 \%)$.

In most anurans, metamorphosis is accompanied with major habitat changes, which also impacts the olfactory system. We labelled glomeruli of the pipid Xenopus tropicalis and the dendrobatid Dendrobates tinctorius in different larval stages during metamorphosis and in early postmetamorphotic animals (Figure 3). In premetamorphotic tadpoles, the glomerular clusters of the left and the right $\mathrm{MOB}$ are separated at the interhemispheric midline (top images, Figure 3 ). At that stage, tadpoles of $D$. tinctorius already have a prominent dorsal glomerular region that extends towards the midline, while this region is less visible in $X$. tropicalis tadpoles. During metamorphosis, the dorsal glomerular regions of both sides start to medially fuse in both examined species (middle images, Figure 3). The ventral clusters (LC; IC; MC) are still present and remain unchanged. In both species, a bundle of axons bypasses the glomerular clusters, terminates in higher brain centers, or crosses the midline more caudally (white arrows, Figure 3). After the completion of metamorphosis (bottom images, Figure 3), the ventral clusters in $X$. tropicalis are unchanged, while they are reduced in $D$. tinctorius (asterisks, Figure 3 ). The LC is still clearly discernable in both species, while especially the IC and MC of $D$. tinctorius are not clearly delineated anymore. 


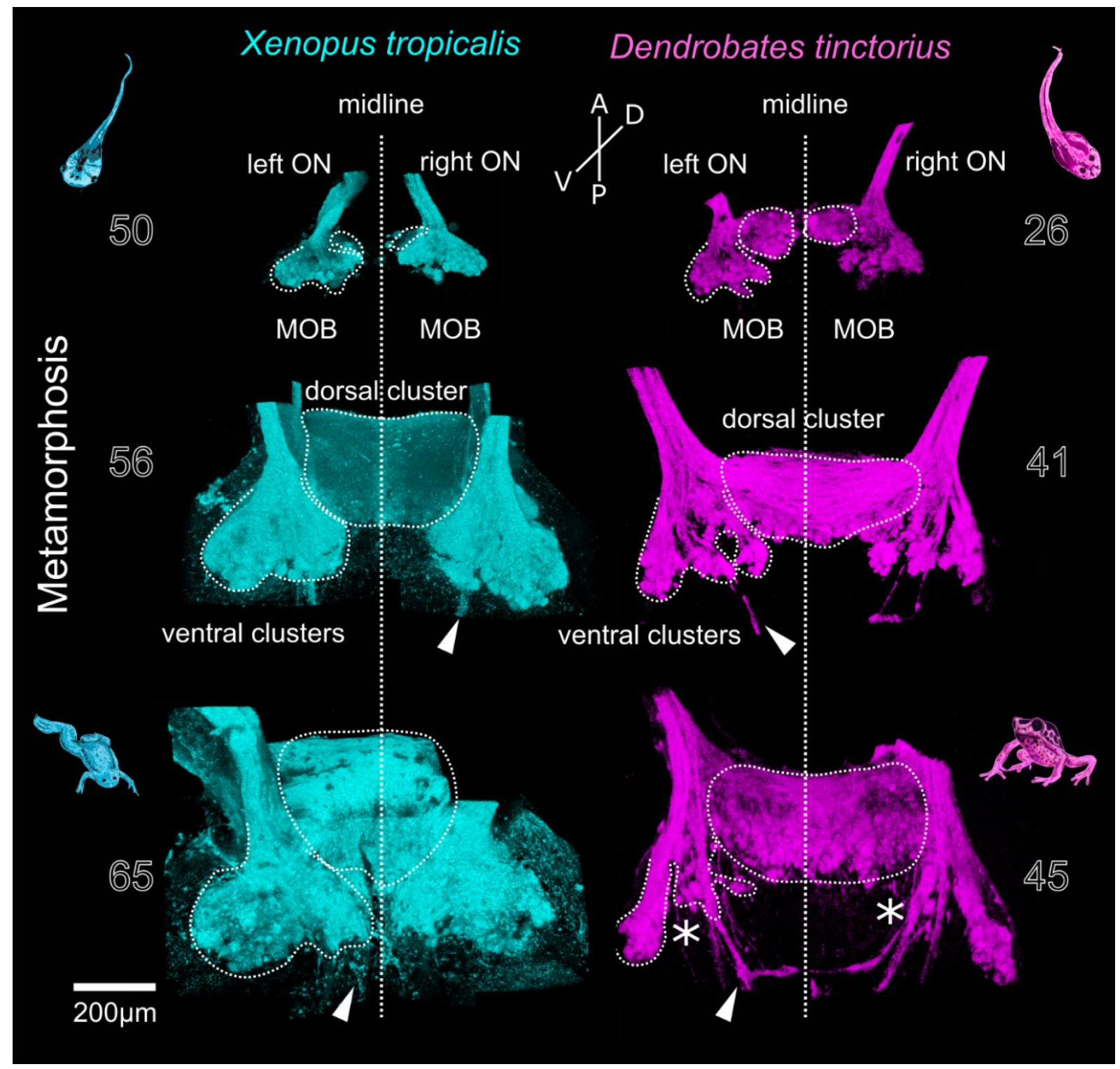

Figure 3 Metamorphotic changes of glomerular clusters in Xenopus tropicalis and Dendrobates tinctorius. Before metamorphosis (top), the left and right glomerular projections in the MOB are separated at the midline (vertical line). During metamorphosis, the dorsomedial components form an unpaired dorsal cluster (middle). In the late phases of metamorphosis (bottom), the ventral glomerular clusters in $X$. tropicalis are unchanged, while they are reduced in $D$. tinctorius (asterisks). Arrowheads - extrabulbar fibers. The numbers next to the images indicate the developmental stages after Nieuwkoop and Faber for $X$. tropicalis and Gosner for D. tinctorius. A anterior, $\mathrm{P}$ posterior, D dorsal, $\mathrm{V}$ ventral, ON olfactory nerve, MOB main olfactory bulb. 


\section{Discussion}

During vertebrate evolution, a trend towards segregation into olfactory subsystems is apparent. Mammals possess several anatomically and molecularly distinct olfactory organs that project to different glomerular regions of the olfactory bulb (Bear et al., 2016; Munger et al., 2009). In fishes, ORNs expressing different odorant receptors are intermingled in a single olfactory surface (Hamdani and Døving, 2007). ORNs expressing receptors belonging to the same gene family often project to segregated glomerular clusters or regions in the olfactory bulb, putatively constituting functionally distinct odorant processing streams (Baier and Korsching, 1994; Braubach et al., 2012; Frontini et al., 2003; Green et al., 2017; Hamdani, 2001a; Hansen et al., 2003, 2004; Sato, 2005). In anurans, detailed analysis of the glomerular array is only available from the African Clawed frog Xenopus laevis (Gaudin and Gascuel, 2005; Manzini et al., 2007). Here we show that the organization of glomerular clusters is remarkably conserved between six distantly related anuran species despite a quite diverse ecomorphology of the examined tadpoles.

In larval $X$. laevis, glomerular clusters have been associated with a lateral and medial odorant processing stream (Gliem et al., 2013; Manzini et al., 2002). The medially located glomeruli in the MOB of $X$. laevis tadpoles are putatively innervated by ciliated ORNs expressing OR-type odorant receptors linked to $G_{\alpha / \text { olf }}$ and using the cAMP transduction pathway. The medial stream shows responses to alcohols, aldehydes and ketones. (Gliem et al., 2013). The lateral cluster on the other hand is highly responsive to amino acid stimulation and expresses $G_{\alpha / o}$ and $G_{\alpha / i}$ linked to a cAMP independent transduction pathway (Gliem et al., 2013; Manzini and Schild, 2003). Vomeronasal-type receptors (V1Rs or V2Rs), as well as trace-amine-associated receptors (TAARs) have been proposed to mediate this lateral stream (Date-Ito et al., 2008; Gliem et al., 2013; Syed et al., 2013). Even though these pathways are described quite in detail, their functional and behavioral significance is so far unknown. Amino acids are generally known as a potent food stimulus in fishes (Hamdani and Døving, 2007) and a lateral processing stream linked to feeding behavior has been identified in the olfactory bulb of the carp (Hamdani, 2001a). Whether the lateral glomerular cluster in tadpoles also mediates feeding or foraging behavior remains to be elucidated (Terni et al., 2018).

Tadpoles of the examined species vary extensively in their feeding behavior, as they have adapted to a variety of microhabitats by differentiating into several morphotypes (Orton, 1953; Roelants et al., 2011). Earlier diverging frogs, like most pipids, rely on a large buccopharyngeal space to pump water through their body (Orton's morphotype I), trapping mostly algae or other small food particles (Seale, 1982). However, derived phenotypes are more specialized, where the development of keratinized mouthparts enables tadpoles of Orton's morphotype IV to rasp food particles off the ground or prey animals (Altig and Johnston, 1989; Roelants et al., 2011). For example, tadpoles of many dendrobatid frogs like Ranitomeya variabilis inhabit small temporary pools in bromeliads or leave axils, which often is linked to a scarcity of food resources (Altig and McDiarmid, 1999; Brown et al., 2008) and facultative cannibalism (Masche et al., 2010). In addition to their feeding behavior, there are major differences in social interactions. While tadpoles of Xenopus or Rhinella arenarum tadpoles are often seen forming schools for protection against predators (Altig and McDiarmid, 1999; Wells, 2007), Dendrobates 
tinctorius tadpoles engage in aggressive behavior against conspecifics (Fischer et al., 2020) and are transported by their parents if the food resources in the pool are exhausted (Altig and McDiarmid, 1999; Brown et al., 2008; Roland et al., 2015). While tadpoles rely on olfaction for foraging and kin recognition (Veeranagoudar et al., 2004; Villinger and Waldman, 2005; Waldman, 1991), it is currently unclear whether the olfactory system shows adaptation to specific ecological niches.

$R$. arenarum, like other anuran larvae also has a part of their main olfactory epithelium exposed to the buccal cavity (Benzekri and Reiss, 2012; Jermakowicz et al., 2004; Jungblut et al., 2017). This buccal exposed epithelium is hypothesized to be important to assess food quality in species that actively scrape food off the substrate, and is absent in suspension feeders like $X$. laevis (Jungblut et al., 2017). The glomerular projection target of this buccal exposed epithelium is currently unknown. It takes up approx. $15-20 \%$ of the entire epithelial volume in R. arenarum tadpoles (Jungblut et al., 2017) and is present in Scinax granulatus like in other hylid tadpoles (Jungblut et al., 2017; Jungblut personal observation). The presence of the buccal exposed epithelium in tadpoles of the two dendrobatid species tadpoles to our knowledge. Despite the eco-morphological difference and differences in the structure of the peripheral olfactory organ of the examined species, the spatial organization of glomeruli in the MOB of all examined tadpoles was remarkably similar. It is however still unclear, whether the anatomically similar clusters are also functionally or molecularly equivalent. In larval $R$. arenarum, both $\mathrm{G}_{\alpha / \mathrm{o}}$ and $\mathrm{G}_{\alpha / \text { off }}$ are expressed in $\mathrm{MOB}$ glomeruli, but no clear segregation could be observed, in contrast to $X$. laevis tadpoles (Jungblut et al., 2009). It seems possible that the glomerular clusters are anatomically conserved but innervated by ORNs expressing receptors belonging to different receptor families, thus detecting different odorant cues. Whether the small variation in the relative volume of the described glomerular clusters e.g. the relatively bigger DC in the Neobatrachians could be attributed to the presence of the buccal exposed epithelium or other ecological differences needs further experimental evidence.

After metamorphosis, the anatomy of the nose of different frog species is more variable than at the larval level (Helling, 1938). The larval MOE in the principal nasal cavity consisting of microvillous and ciliated ORNs transforms into the adult 'air-nose', solely consisting of ciliated olfactory ORNs (Bloom, 1954; Föske, 1934; Hansen et al., 1998; Reiss and Eisthen, 2008). In pipid frogs, a sensory epithelium consisting of both microvillous and ciliated ORNs forms in the middle cavity (Hansen et al., 1998) and seems to be a molecular and functional copy of the larval MOE (Hansen et al., 1998; Syed et al., 2017). In other anurans, a small patch of 'aquatic' epithelium forms at the anterior bottom of the principal cavity, the recessus olfactorius, a putative homologue to the pipid middle cavity (Helling, 1938; Nowack et al., 2013). The middle cavity in these species is non-sensory (Reiss and Eisthen, 2008).

In adult Xenopus laevis, ORNs located in the de novo formed middle cavity epithelium project to the ventrally located glomerular clusters, while the remodeled principal cavity connects to the dorsomedial glomeruli in the MOB (Gaudin and Gascuel, 2005; Reiss and Burd, 1997a). The ventrally located glomerular clusters have been shown to remain anatomically (Gaudin and Gascuel, 2005) and functionally (Weiss et al., unpublished data) stable during metamorphosis, constituting a channel for waterborne olfaction. The dorso-medial region grows extensively and fuses at the midline, forming a 
single dorsal MOB (Gaudin and Gascuel, 2005). We show that the development of the dorso-medial portion of the MOB is similar between Xenopus tropicalis and Dendrobates tinctorius (Figure 3). Contrastingly, the ventrally located glomerular clusters are reduced in $D$. tinctorius after metamorphosis. Since $D$. tinctorius juveniles and adults are terrestrial, it is not clear to what extent they still rely on aquatic olfaction, in contrast to the fully aquatic Xenopus. Comparative data from different terrestrial and aquatic adults are needed to fully understand adaptive traits in the glomerular organization.

In conclusion, we show that the organization of glomerular clusters in eco-morphologically diverse tadpoles of distantly related species is remarkably conserved. It remains to be shown if the segregation into glomerular clusters also represents functionally distinct subsystems and if the small inter-species variability reflects possible adaptation to specific microhabitats.

\section{Acknowledgements}

We thank all members of the Manzini laboratory for discussion, Anja Schnecko for technical assistance and Eva Fischer for support to set up experiments.

\section{Competing interests}

No competing interests.

\section{Author Contributions}

Conceptualization, L.W., T.H., I.M.; Investigation, Formal Analysis, Visualization and Writing: L.W.; Review \& Editing, L.W., L.D.J., A.G.P., L.A.O., T.H., I.M.; Funding Acquisition and Resources, L.D.J., A.G.P., L.A.O., T.H., I.M.; Supervision, T.H., I.M.

\section{Ethics approval}

All experiments followed the guidelines of Laboratory animal research of the Ethics Committee of the University of Buenos Aires (CD: 316/12, Protocol \#22), Justus-Liebig-University Gießen, (GI 15/7, 932_GP) and Stanford University (APLAC-33016).

\section{Funding}

This work was supported by DFG Grant 4113/4-1, the cooperation program CONICET-DFG-MINCYT 23120160100031CO, UBACYT 20020170200191BA and by Award Number S10RR02557401 from the National Center for Research Resources (NCRR). Its contents are solely the responsibility of the authors and do not necessarily represent the official views of the NCRR. L.W. was granted a travel fellowship (JEBTF-180809) by the Company of Biologists. 


\section{Chapter 4: Development of neuronal circuits for aquatic and aerial olfaction during amphibian metamorphosis}

\section{Authors and affiliations}

Lukas Weiss $^{1 *}{ }^{*}$, Paola Segoviano Arias ${ }^{12}$, Thomas Offner ${ }^{1}$, Sara Joy Hawkins ${ }^{1}$, Thomas Hassenklöver ${ }^{1}$ and Ivan Manzini'

1 Institute of Animal Physiology, Department of Animal Physiology and Molecular Biomedicine, JustusLiebig-University Giessen, 35392 Giessen, Germany

2 Current address: Max Planck Research Unit for Neurogenetics, 60438 Frankfurt, Germany

*Correspondence lukas.weiss@physzool.bio.uni-giessen.de (L.W.)

\section{Contribution statement}

I conducted all tracing and sparse cell labelling experiments presented in Figures 1, 2, 4 and 5, analyzed and visualized all the data presented in these figures and wrote the manuscript. I supervised the calcium imaging experiments conducted by P.S.A. during her M.Sc. thesis. Calcium imaging data was analyzed by T.O, behavioral experiments were conducted and analyzed by S.J.H. I conceptualized the study together with T.H. and I.M. L.W., T.O., S.J.H., T.H., I.M were involved in reviewing and editing the manuscript.

\section{Key words}

Metamorphosis, olfactory system, anura, glomeruli, aquatic olfaction, neuronal circuit 


\begin{abstract}
The anuran olfactory system undergoes substantial adaptive changes during the metamorphotic shift from aquatic tadpole to terrestrial adult. Despite being fully aquatic, the Clawed frog Xenopus laevis has a nose adapted to detect both water- and airborne odorants. Xenopus tadpoles only possess a 'water nose' in their principal nasal cavity. During metamorphosis, a second sensory epithelium in the middle cavity - the adult 'water nose' - develops, while sensory neurons in the principal cavity are replaced to form an adult 'air-nose'. Here we investigated the changes in neuronal connections between the nose and the main olfactory bulb during metamorphosis. Olfactory receptor neurons in the larval principal cavity innervate glomeruli in the ventral main olfactory bulb. These projections are gradually replaced by receptor neuron axons from the newly formed middle cavity epithelium. During this complete innervation shift, the spatial segregation into medio-dorsal cAMP-dependent projections and ventro-lateral CAMP-independent, amino acid sensitive glomeruli and behavioral responses to amino acid persists. Receptor neuron axons from the remodeled principal cavity project to the dorsomedial glomeruli, which grow to occupy around $70 \%$ of the entire glomerular volume. This part of the bulb is fused at the midline, allowing extensive crossing over of axons coming in from the left and right nose. Postsynaptic projection neurons in the dorsal olfactory bulb are connecting to more glomeruli than in the ventral olfactory bulb, putatively integrating input from the left and right nostril. The differences in the circuit configuration in the dorsal and ventral olfactory bulb suggest distinct ways of odor processing which might be adaptive features to the aquatic or aerial odor space.
\end{abstract}

\title{
Introduction
}

Habitat has a great impact on the sense of smell. The first vertebrates to be confronted with the obstacle of detecting odors both in water as well as in air were the direct ancestors of the modern amphibians. Their metamorphosis from aquatic larvae to more or less water-independent adults provides the advantage to study both aquatic as well as aerial olfaction in the same animal (Duellman and Trueb, 1994; Reiss and Eisthen, 2008; Wells, 2007).

Tadpoles of most anuran amphibians share a similarly structured olfactory periphery, consisting of the main olfactory epithelium in the principal nasal cavity (PC), a vomeronasal organ (VNO), as well as some minor diverticula. This is well documented for all groups of anurans: Archaeobatrachians (Benzekri and Reiss, 2012), Mesobatrachians (Manzini and Schild, 2010) and Neobatrachians (Jermakowicz et al., 2004; Jungblut et al., 2011, 2017; Nowack and Vences, 2016; Quinzio and Reiss, 2018). During metamorphosis, a tripartite chamber system forms, consisting of the main olfactory epithelium in the PC, a middle cavity (MC) lined with non-sensory epithelium in most species and the VNO (Helling, 1938; Reiss and Eisthen, 2008). While the larval system (PC and VNO) is associated with the detection of waterborne odors, the sensory epithelium in the adult PC is specialized for sampling air (for review see Reiss \& Eisthen, 2008). 
In contrast to the majority of anurans, the pipid frog Xenopus laevis evolved a secondarily aquatic lifestyle. The almost fully water-dwelling adults (Reiss and Eisthen, 2008; Wells, 2007) only occasionally move overland (Measey, 2016; Du Plessis, 1966). The special ecology of adult Xenopus is reflected in the presence of a specialized 'water-nose' in the MC, which starts to form around the premetamorphotic stage 51 after Nieuwkoop and Faber (Dittrich et al., 2016; Föske, 1934; Hansen et al., 1998; Higgs and Burd, 2001; Nieuwkoop and Faber, 1994; Reiss and Burd, 1997b, 1997a). The epithelium in the adult MC exhibits strong similarities with the larval PC. It contains both major types of olfactory receptor neurons (ORNs, ciliated and microvillous; Hansen et al., 1998), is responsive to common waterborne olfactory stimulants like amino acids (Sorensen and Caprio, 1998; Syed et al., 2017) and expresses a similar set of olfactory receptors (Freitag et al., 1995, 1998; Syed et al., 2013, 2017). During metamorphosis, major remodeling occurs in the larval PC caused by massive cell death and replacement of ORNs (Dittrich et al., 2016; Hansen et al., 1998; Higgs and Burd, 2001). The remodeled adult PC is eventually composed of only ciliated ORNs and expresses olfactory receptors putatively responsive to airborne odorants (Freitag et al., 1995; Mezler et al., 1999, 2001), thus assuming the role of the adult 'air nose' (Föske, 1934; Hansen et al., 1998; Syed et al., 2017). In contrast, the VNO does not seem to change significantly during metamorphosis in regard to its cellular composition or function (Dittrich et al., 2016; Hansen et al., 1998).

The segregation of the olfactory periphery also translates to the level of the olfactory bulb. In larval Xenopus laevis, the ORN axons originating in the PC project to olfactory glomeruli in the ventral portion of the main olfactory bulb (vMOB) via the olfactory nerve (ON) (Manzini and Schild, 2010; Reiss and Burd, 1997b). Receptor neurons in the VNO project towards the accessory olfactory bulb (AOB) (Reiss and Eisthen, 2008). Around stage 52, the first ORN axons originating from the newly forming $M C$ epithelium reach the glomeruli in the $\mathrm{VMOB}$ where they overlap with the existing axons from the PC (Reiss and Burd, 1997a). Later during metamorphosis, the $\mathrm{NMOB}$ is exclusively innervated by axons originating in the MC (Reiss and Burd, 1997b), while the ORN axons from the PC are now projecting to newly formed glomeruli in the dorsal region of the main olfactory bulb (dMOB) (Gaudin and Gascuel, 2005; Reiss and Burd, 1997b). This bipartition is also supported by distinct lectin binding profiles of ORN axons (Franceschini et al., 1992; Hofmann and Meyer, 1991; Key and Giorgi, 1986).

The glomeruli in the $\mathrm{VMOB}$ are anatomically grouped into segregated clusters that stay morphologically intact during metamorphosis (Gaudin and Gascuel, 2005). In tadpoles, these clusters are functionally grouped into two major processing streams: laterally located glomeruli use a second messenger cascade independent of CAMP, while more medially located glomeruli use CAMP as a second messenger (Gliem et al., 2013). A substantial portion of the lateral glomeruli are responsive to amino acids and putatively innervated by microvillous ORNs (Gliem et al., 2013), while the medially located glomeruli are connected to ciliated ORNs and responsive to alcohols, aldehydes and amines (Gliem et al., 2013). It is unclear, whether this functional pattern is preserved during metamorphosis and how it is influenced by the innervation shift from larval PC to adult MC. In contrast to the VMOB, the $\mathrm{dMOB}$ is not present in the tadpole and grows extensively until the end of metamorphosis (Reiss and Burd, 1997b). So far, no processing streams, glomerular clusters, or features of the dMOB circuit have been described in detail. 
In the present work we describe the innervation shift in the $\mathrm{VMOB}$ from larval PC to adult MC on an anatomical, functional, and behavioral level. Despite major remodeling processes during metamorphosis, the two separate processing streams in the 'water-smelling'-system in the vMOB seem conserved up until metamorphotic climax. Similarly, behavioral responses to amino acids do not change during metamorphosis. ORN axons projecting to the $\mathrm{dMOB}$ progressively fuse at the midline during metamorphosis, forming an unpaired projection field. We found a difference in connectivity pattern of the postsynaptic neurons in the $\mathrm{VMOB}$ and $\mathrm{dMOB}$, with a multi-glomerular pattern being more prevalent in the $\mathrm{dMOB}$. These features of the dorsal 'air-system' point towards a putatively higher degree of integration between left and right and across several glomeruli. The parallel existence of the $\mathrm{VMOB}$ and the $\mathrm{dMOB}$ circuits suggests different adaptive features of aquatic and aerial odor processing in amphibians.

\section{Material and Methods}

\section{Animals and tissue preparation}

All animals used in this study were wild type or albino Xenopus laevis (both sexes), kept and bred at the University of Giessen at a water temperature of $20^{\circ} \mathrm{C}$ in water tanks with constant water circulation. Developmental stages were defined according to Nieuwkoop and Faber (Nieuwkoop \& Faber, 1994). Before experimental procedures, the animals were anaesthetized using $0.02 \% \mathrm{MS}-222$ (ethyl 3-aminobenzoate methanesulfonate; TCl Germany) in tap water. For tissue preparation, anaesthetized animals were killed by severing the transition between brainstem and spinal cord and a tissue block containing the noses and the rostral part of the telencephalon was removed. For higher staged animals, the $\mathrm{ON}$ was cut close to the noses and the entire brain was taken out of the cartilage. All animal procedures were performed in accordance with the guidelines of Laboratory animal research of the Institutional Care and Use Committee of the University of Gießen, (GI 15/7, 932_GP).

\section{Tracings of olfactory sensory neurons via WGA and electroporation}

For bulk loadings of olfactory projections from the nose to the $M O B$, we incubated the olfactory epithelia with approx. $3 \mu \mathrm{l}$ of fluorophore-coupled wheat germ agglutinin (WGA Alexa Fluor 488 or 594 conjugate, Thermo Fisher) diluted at a concentration of $10 \mathrm{mg} / \mathrm{ml}$ in saline Frog Ringer (in $\mathrm{mM}: 98$ $\mathrm{NaCl}, 2 \mathrm{KCl}, 1 \mathrm{CaCl}_{2}, 2 \mathrm{MgCl}_{2}, 5$ glucose, $5 \mathrm{Na}$ - pyruvate, 10 Hepes, pH 7.8) for 10 minutes. For bulk electroporations we placed dried dye crystals of fluorophore-coupled dextrans (Alexa dextran 488, 594 or Cascade Blue dextran, $10 \mathrm{kDa}$, Thermo Fisher; $3 \mathrm{mM}$ in frog Ringer) in the nostrils and applied six electric square pulses using two platinum electrodes $(15 \mathrm{~V}, 25 \mathrm{~ms}$ duration at $2 \mathrm{~Hz}$ with alternating polarity) to each nostril (for detailed protocol see Weiss et al., 2018).

\section{Sparse cell electroporation in the MOE and the MOB}

We sparsely labeled ORNs in the nasal epithelia of $P C / M C$ and projection neurons in the $\mathrm{VMOB} / \mathrm{dMOB}$ using micropipettes pulled from borosilicate glass capillaries (Warner instruments, resistance 10-15 
$M \Omega$ ) filled with fluorophore-coupled dextrans (Alexa dextran 488 and 594). The dye filled capillaries were mounted on the headstage of an Axoporator 800A equipped with a wire electrode (Axon instruments, Molecular Devices) and approached to the respective cell types in the MOE or the OB using a micromanipulator. A $500 \mathrm{~ms}$ train of square voltage pulses $(50 \mathrm{~V}$, single pulse duration $300 \mu \mathrm{s}$ at $200-300 \mathrm{~Hz}$ ) was applied.

For ORN labelling, the animals were first anaesthetized and sparse cell electroporation was repeated at multiple locations using Alexa 488 dextran in the MC and Alexa 594 dextran in the PC to trace their respective projections during metamorphotic stages. After the procedure, animals were left to recover for at least 24 hours prior to image acquisition (Hassenklöver and Manzini, 2014). Sparse labelling of projection neurons in the $\mathrm{MOB}$ was conducted in the tissue block containing the olfactory system. We fixed the bulb and the caudal portion of the ON under a platinum grid stringed with nylon threads, approached the micropipette and applied the voltage pulse trains as described above (detailed protocol Weiss et al., 2018).

\section{Image Acquisition and processing of morphological images}

Images were acquired as virtual image stacks with multiple color detection channels and a z-resolution of 1-3 $\mu \mathrm{m}$ using multiphoton microscopy (Nikon 1AR-MP) at an excitation wavelength of $780 \mathrm{~nm}$. We used ImageJ (Schindelin et al., 2012; RRID:SCR_003070) to adjust brightness and contrast of the image stacks and applied a median filter to remove pigmentation derived artifacts in some images. Separate images were stitched together where necessary (Preibisch et al., 2009). For thresholding analyses conducted on the image-stacks, we eliminated tissue-derived autofluorescence by subtracting the blue-wavelength color channel (when no blue-emitting dye was introduced into the tissue). Images are presented as maximum intensity projections along the z-axis or in 3D using the 3D-viewer implemented in ImageJ.

\section{Functional calcium imaging and data processing}

For functional calcium imaging in the axon terminals of the $\mathrm{VMOB}$, we loaded the ORNs with a Calcium sensitive dextran coupled dye (Cal 520 dextran conjugate, $10 \mathrm{kDa}$, AAT Bioquest; $3 \mathrm{mM}$ in frog Ringer) via electroporation as described above. After killing the animal, we cut out the tissue block containing the noses and the anterior part of the brain and removed tissue around the sensory epithelia. The block was positioned on the stage of the multiphoton microscope using a platinum grid stringed with nylon threads. An outflow funnel (Milli Manifold; ALA Scientific) connected to a gravity-fed multichannel perfusion system (ALA-VM-8 Series; ALA Scientific) was positioned in front of the MOE and a constant Ringer through-flow was established. Ringer was constantly removed from the recording chamber via a syringe needle connected to a peristaltic pump via silicone tubing (Offner, 2020). Volumetric recordings of the glomerular projections in the $\mathrm{VMOB}$ were made using the multiphoton microscope (780 excitation wavelength) with acquisition rates of 30-40 planes per second at a zresolution of 3-5 $\mu \mathrm{m}$. Stimuli were applied for 5 seconds duration in an interval of 60 seconds and repeated at least twice. The mixes consisted of the following single components: I) Control stimulus Frog Ringer solution; II) Amino acid mix: I-proline, I-valine, I-leucine, I-isoleucine, I-methionine, glycine, 
I-alanine, I-serine, I-threonine, I-cysteine, I-arginine, I-lysine, I-histidine, I-tryptophan, I-phenylalanine; III) Amine mix: 2-phenylethylamine, tyramine, butylamine, cyclohexylamine, hexylamine, 3methylbutylamine, N,N-dimethylethylamine, 2-methylbutylamine, 1-formylpiperidine, 2methylpiperidine, N-ethylcyclohexylamine, 1-ethylpiperidine, piperidine; IV) Bile acid mix: taurocholic acid, cholic acid, glycholic acid, deoxycholic acid; V) ATP; VI) Odorant mix: amino acids, amines, bile acids; VII) Forskolin. All chemicals were purchased from Sigma Aldrich. The mixes were freshly prepared before the experiments from frozen aliquots at a concentration of $100 \mu \mathrm{M}$. Forskolin was concentrated at $50 \mu \mathrm{M}$.

A piecewise-rigid motion correction algorithm was applied to remove motion artifacts (Pnevmatikakis and Giovannucci, 2017) followed by further denoising and deconvolution steps using the CalmAn toolkit for calcium imaging data (Friedrich et al., 2017; Giovannucci et al., 2019; Pnevmatikakis et al., 2014, 2016). Denoised volumetric recordings are presented as maximum intensity projections along the z-axis of fluorescence intensity difference maps (Figure 3a). Intensity difference maps resulted from the difference between peak fluorescence intensity of post-stimulus responses (averaged from three timeframes) and baseline fluorescence prior to stimulus onset (averaged from five frames) (Offner, 2020). Glomerular response profiles were defined according to (Offner, 2020). The relative position of responding glomeruli (Figure $3 \mathrm{~b}$ ) was measured in relation to two manually defined points at the medial and lateral edge of the glomerular projections in each VMOB. Responsive regions outside of this defined lateral-medial area were discarded. The relative positions were pooled for all responding glomeruli in all analyzed samples and significance was tested with a Mann-Whitney-U test.

\section{Analysis of ORN projections to the MOB}

The relative volumes of the ventral and dorsal portions of the ORN projections into the MOB (presented in Figure 1) were measured using the Segmentation Editor implemented in ImageJ (Schindelin et al., 2012) and analyzed using Python. The data show the percentual share of ORN projections in the $\mathrm{AMOB}$ and the $\mathrm{VMOB}$ relative to the volume of the entire ORN projections ( $\mathrm{AMOB}+$ vMOB). Projections in both hemispheres were summed up for this analysis.

For the analysis of $\mathrm{MC}$ and $\mathrm{PC}$ projections to the $\mathrm{MOB}$ (shown in Figure 2), we reduced the image stacks to a maximum intensity projection along the $\mathrm{z}$ axis and separated image planes containing the $\mathrm{VMOB}$ and the $\mathrm{dMOB}$. Projections from the MC and PC were detected in two separated color channels. Images were then binarized using the Maximum Entropy thresholding method implemented in ImageJ (Kapur et al., 1985). The percentual share of pixels containing ORN fibers from the MC and PC was calculated in relation to the total number of pixels containing fluorescent signal. This analysis was conducted separately for the $\mathrm{dMOB}$ and the vMOB.

To calculate the overlap of ORN axonal projections originating in the left and right OE (Figure 4), we applied a median filter with a window size of 10 pixels to the virtual two-color channel image stacks in ImageJ and subsequently binarized the image applying Huang's fuzzy thresholding method (Huang and Wang, 1995). Pixels containing signal in both color channels of the image were summed up and their 
percentual share of the entire structure was calculated. Separate analyses for $\mathrm{AMOB}$ and $\mathrm{VMOB}$ were conducted.

\section{Reconstruction and analysis of labelled projection neurons}

The morphology of projection neurons in the MOB labelled via electroporation was semi-automatically reconstructed from the image stack using Vaa3D (Peng et al., 2010; RRID:SCR_002609). Branching- and endpoints of the projection neurons were defined and translated into a hierarchical 3D tree-structure with the soma of the neuron as the root-point. Each segment of the neuron-tree has a single parent segment that it connects to. Each projection neurons connects to at least one olfactory glomerulus with a dendritic tuft. The number of tufts was determined using the DBSCAN algorithm (Density-Based Spatial Clustering of Applications with Noise) implemented in the scikit learn machine learning package written for Python (Pedregosa et al., 2011; Weiss et al., 2020). The algorithm classifies an accumulation of end- and branching points of the neuronal structure as tuft-clusters if there are more than 5 points in spatial proximity $(<15 \mu \mathrm{m})$. All branching- and endpoints that are sparsely distributed in space are classified as blunt endings and not part of a tuft-cluster. The distances of the dendritic tufts to the root of the tree (soma) were measured along the dendritic branches and the distance between two tufts as Euclidean distance in 3D space. The volumes of the tufts were estimated based on the volume of a convex hull of all branching- and endpoints belonging to a single tuft-cluster.

\section{Behavioral assay}

Tadpoles of different developmental stages were placed in a 1-liter water tank which was separated into two areas by a dividing wall along approx. half of its length (Figure 3c). The two areas were freely accessible to the tadpoles. Tadpoles were left to swim without stimulus application for a $2 \mathrm{~h}$ habituation period and the average time they spent per visit to the two areas at either side of the dividing wall was recorded. After the habituation period, $5 \mathrm{ml}$ of an amino acid mix $(100 \mu \mathrm{M}$ at the application entry point) were applied through a gravity feed to one of the two areas, while a control stimulus ( $5 \mathrm{ml}$ water) was applied on the other side. The average time the tadpoles spent in the amino acid and water control area per visit were recorded for $20 \mathrm{~min}$ after their first visit to either area using EthoVision tracking software. Tadpoles that did not enter either side within $\mathbf{4 0}$ minutes after stimulus application were not included in the analysis.

\section{Statistics}

Averaged data are presented as mean \pm standard deviation. A least-squares based polynomial regression curve of third degree was fitted through the $\mathrm{VMOB} / \mathrm{dMOB}$ volume data. $A$ linear regression using the RANSAC (Random sample consensus) algorithm (scikit learn, RRID:SCR_002577; Pedregosa et al., 2011) was used to analyze the left/right projection overlap in the dMOB. Statistical significance was tested using a Mann-Whitney-U test. Behavioral data is presented as median values of all experimental animals and significance was assessed using Wilcoxon signed rank test for paired data. 


\section{Results}

\section{The dMOB expands drastically during early metamorphosis}

During metamorphosis, drastic changes take place in the olfactory periphery and the olfactory bulb. A major amount of ORNs undergoes apoptosis (Dittrich et al., 2016; Reiss and Burd, 1997a) and newly formed ORNs integrate into the preexisting functional system. In premetamorphotic larvae (up to stage 54), the olfactory epithelium in the PC innervates the $\mathrm{VMOB}$ (Figure 1a, left). After metamorphosis is completed (stage 66), the PC epithelium connects to a newly formed dorso-medial target area (magenta) and the de novo formed MC epithelium innervates the $\mathrm{VMOB}$ (green, Figure 1b, right). We have monitored the stage-by-stage changes in the projections to the glomeruli in the MOB using WGA neuronal tracings (Figure $1 b$ ).
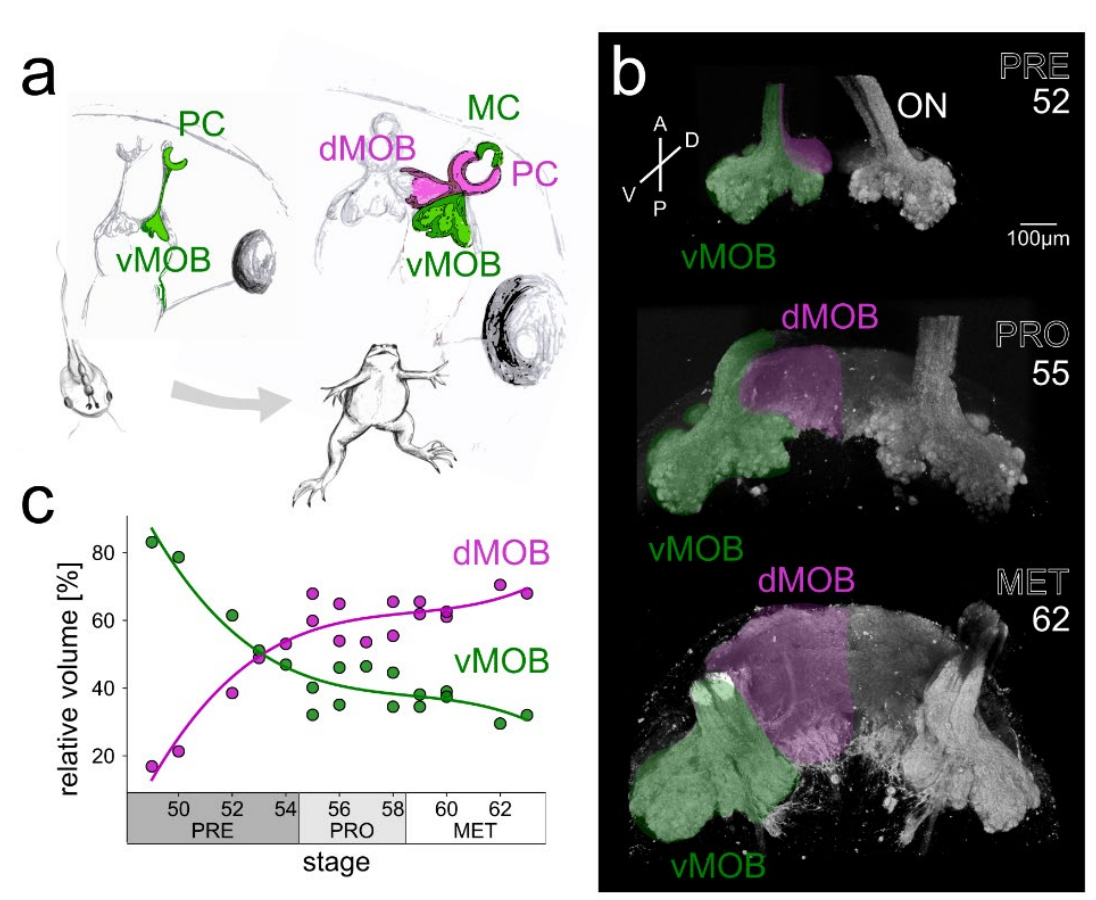

Figure 1 Differential
development of projection
areas in the ventral and dorsal
MOB during metamorphosis.

(a) The schematic shows projections of ORNs residing in the $P C$ innervating the $\mathrm{VMOB}$ (green) in the tadpole. After metamorphosis, the newly formed MC connects to the vMOB (green), while the remodeled $P C$ epithelium innervates the $\mathrm{dMOB}$ (magenta). (b) 3D images of the caudal part of the ONs and the glomerular projections during premetamorphosis (stages up to 54), prometamorphosis (55-58)

and metamorphosis proper (59-64), stained with fluorophore-coupled WGA. Numbers in the panel indicate the stages. The projections in the $\mathrm{VMOB}$ are present in premetamorphosis and retain their morphology during development (magenta). The $\mathrm{dMOB}$ projections start to form around stage 50 and increase dramatically in size until the end of metamorphosis (green). In contrast to the $\mathrm{VMOB}$, the $\mathrm{AMOB}$ is not divided at the interhemispheric midline (c) Percentual share of projection volume in the $\mathrm{VMOB}$ (green) and dMOB (magenta) relative to the total volume during metamorphotic development. Around stage 50, the dMOB only occupies about $20 \%$ of the total glomerular volume and grows to occupy more than $60 \%$ around stage 62 . The relative growth of the $d M O B$ is best be described by a cubic curve $\left(R^{2}=0.9\right)$. A anterior, $D$ dorsal, dMOB dorsal main olfactory bulb, MC middle cavity, MET metamorphosis proper, ON olfactory nerve, $\mathrm{P}$ posterior, $\mathrm{PC}$ principal cavity, PRE premetamorphosis, PRO prometamorphosis, $\mathrm{V}$ ventral, vMOB ventral main olfactory bulb. 
In premetamorphotic tadpoles (stages 49 - 54), glomerular projections on the ventral side of the MOB are clearly discernable (green shaded area in Figure $1 \mathrm{~b}$ ), while only few axonal projections in the dMOB are visible (magenta, Figure 1b). At this stage in development, the two MOB hemispheres are clearly separated. During prometamorphosis (stages 55 - 58), and metamorphosis proper (stages 59 - 65), the VMOB glomerular projections only slightly increase in size, while the $\mathrm{dMOB}$ shows a more massive volume increase (Figure 1 b, middle and bottom). Around stages 53/54, the dMOB fuses at the midline and forms one single dorsal projection field. To understand the relative growth of the two MOB parts, we assessed the percentual share of $\mathrm{VMOB}$ and $\mathrm{dMOB}$ projections during metamorphotic reorganization (Figure 1c). During premetamorphosis, the projections in the VMOB occupied $64.3 \%$ and the $\mathrm{dMOB} 35.7 \pm 16.2 \%$ of the entire axonal projections in the MOB $(n=5)$. The relative volume of the $\mathrm{VMOB}$ progressively decreases to $39.8 \pm 6 \%$ during prometamorphosis $(n=7)$ and $35.1 \pm 3.8 \%$ during metamorphosis proper $(n=6)$. Inversely, the dMOB grows to occupy $64.1 \pm 3.8 \%$ of the entire $\mathrm{MOB}$ projection volume in the late metamorphotic group. The best fit model for our data was a polynomial curve of third degree (least squares method, $R^{2}=0.9$; Figure $1 c$ ). The magenta curve in Figure $1 \mathrm{c}$ shows a steep increase in the relative volume of the dMOB from less than $20 \%$ at stage 50 to $50 \%$ around the end of premetamorphosis (intersection points of the two regression curves, Figure 1c). Subsequently, the $\mathrm{dMOB}$ percentage is rising steadily but with a smaller slope until reaching approx. $70 \%$ in the late metamorphotic stages.

\section{The vMOB is innervated by cells from the new MC and the PC until metamorphotic climax}

While the glomerular structures of the $\mathrm{AMOB}$ are formed the novo, the $\mathrm{vMOB}$ undergoes major transformation processes during metamorphosis. We sparsely labelled ORNs in the newly forming MC (green, Figure 2a) and the PC (magenta, Figure 2a) and imaged their respective axonal projections in the MOВ (Figure $2 b$ ). The ORNs labelled were bipolar neurons with a long dendritic shaft on the apical side of the epithelium and an axon leaving the epithelia on the basal side to join the ON (asterisks, Figure 2a). Since the MC only starts to develop around stage 51 , single/few axons originating in the $M C$ could be detected reaching the $\mathrm{VMOB}$ during premetamorphotic stages (Figure $2 \mathrm{~b}$, left). Most ORN axons innervating glomeruli in the VMOB originated in the PC (magenta). The prometamorphosis is characterized by a VMOB co-innervation between ORN axons originating in the PC and the MC (Figure $2 \mathrm{~b}$, middle). During metamorphosis proper, the $\mathrm{VMOB}$ is solely innervated by axons from the $\mathrm{MC}$. In contrast, the newly forming $\mathrm{dMOB}$ only contains axons originating in the PC (Figure $2 b$, right).

We assessed the dynamic innervation shift in the VMOB by measuring the percentual share of MC- and PC-originating fibers during metamorphosis (Figure 2c). During premetamorphosis $(n=4), 95.5 \pm 4.8 \%$ of the total innervation of the $\mathrm{VMOB}$ originates in the PC. In prometamorphotic stages $(n=5)$ we found $55.2 \pm 30 \%$ of innervation originating in the PC, while $44.8 \pm 30 \%$ of the innervation originated in the MC. The $V M O B$ in later metamorphotic animals is innervated mostly by MC axons $(82.1 \pm 26.6 \%, n=$ 5). Around stage 58 , the percentual share between $P C$ and $M C$ fibers reaches approx. 50\%. Starting from stages 60/61, the VMOB seems to be exclusively innervated by the MC (Figure 2b, c). Contrastingly, the dMOB is only innervated by $P C$ axons $(96.7 \pm 4.4 \%, n=14$ across all stages; Figure 2c, right plot). 

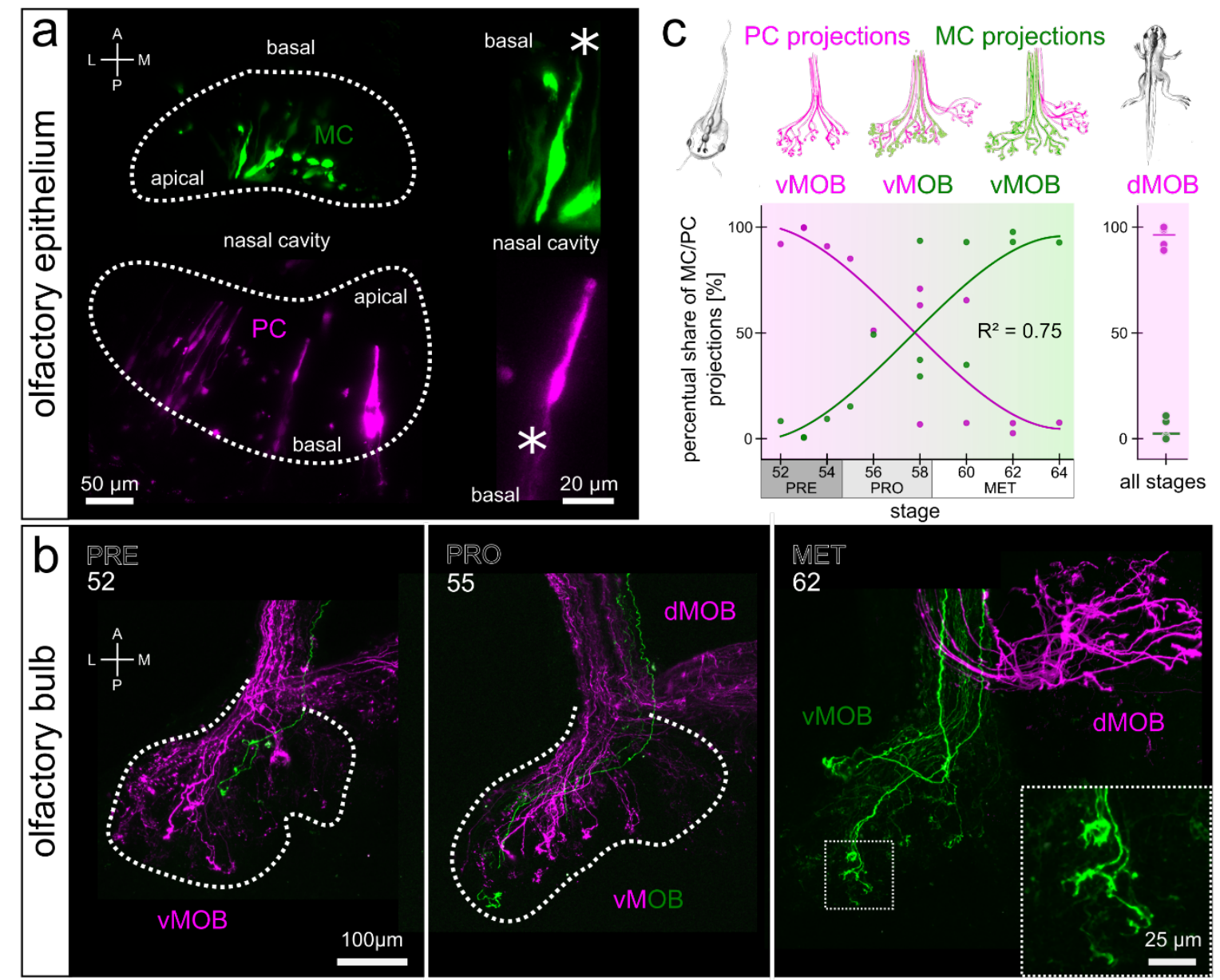

Figure 2 Innervation shift in the vMOB from PC axons to MC axons and PC projections to the dMOB. (a) Labelling of ORNs in the sensory epithelia of MC (green) and the PC (magenta) using different dextran coupled fluorophores via sparse cell electroporation. Close ups show single bipolar ORNs extending their dendrite into the nasal cavity. Asterisks indicate the direction of the axons. (b) Representative tracings of axonal projections in the $V M O B$ of tadpoles during metamorphosis. Numbers indicate the respective stages. During premetamorphosis, the first ORN axons originating in the newly forming MC reach the glomeruli (left). In prometamorphotic animals, both $\mathrm{MC}$ and $\mathrm{PC}$ axons are innervating glomeruli in the $\mathrm{VMOB}$ (middle). During metamorphosis proper, the $\mathrm{VMOB}$ is solely innervated by axons originating in the $\mathrm{MC}$, the newly formed $\mathrm{dMOB}$ only from PC fibers (right). Dotted lines indicate the outline of glomerular projections in the VMOB, the box on the right is a close up of MC fibers. (c) Schematic of innervation shift in the VMOB (top). Percentual share of MC (green) and PC (magenta) innervation in the $\mathrm{VMOB}$ during metamorphotic development follows a curve of third degree $\left(R^{2}=0.75\right.$; right plot). The dot plot on the right shows the same data for the $d M O B$, which is only innervated by $\mathrm{PC}$ axons throughout development. A anterior, dMOB dorsal main olfactory bulb, $L$ lateral, $M$ medial, MC middle cavity, MET metamorphosis proper, ON olfactory nerve, P posterior, PC principal cavity, PRE premetamorphosis, PRO prometamorphosis, $\mathrm{VMOB}$ ventral main olfactory bulb.

\section{Two parallel odor processing streams are present during rewiring of the vMOB.}

ORN axonal projections from the PC epithelium to the glomeruli in the VMOB are gradually replaced by $\mathrm{MC}$ projections during metamorphotic remodeling. We investigated if this replacement also 

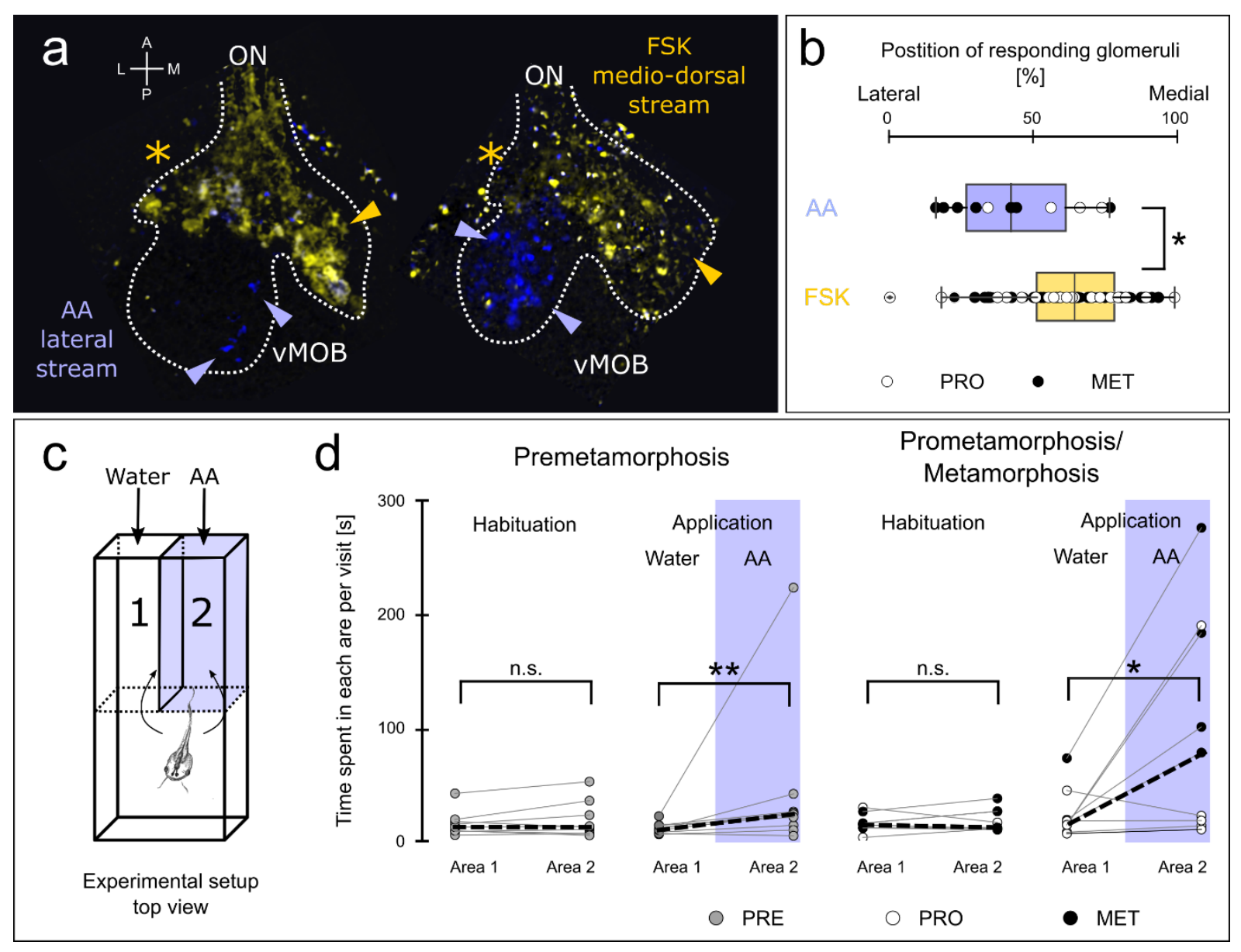

Prometamorphosis/ Metamorphosis

Figure 3 Odorant processing streams in the metamorphotic vMOB and behavioral responses to amino acids. (a) Difference maps showing the increase in fluorescent signal after application of amino acid mix (blue) and forskolin (yellow). Representative vMOBs of prometamorphotic tadpoles (stages 57 and 58) are shown. The white dotted lines indicate the outline of $\mathrm{VMOB}$ glomerular projections. Segregation into a lateral forskolinindependent processing stream responding to amino acids, and a medio-dorsal forskolin-dependent odorant processing stream is visible. (b) Relative position from lateral (0\%) to medial (100\%) of cAMP-independent glomeruli tuned to amino acids and the positive control (blue) and cAMP-dependent glomeruli activated by forskolin (yellow). The population of amino acid responsive glomeruli $(n=11)$ is significantly more lateral in comparison to the forskolin responses ( $n=77 ; p<0.05$ ). Lateral cAMP-dependent/forskolin-activated glomeruli are located dorso-laterally (yellow asterisks). Glomeruli of eight animals were pooled together for this analysis. (c) Experimental setup in top view. Tadpoles could freely move between the two areas separated by the dividing wall. After two hours of habituation, a water control stimulus was applied in area one and amino acids in area two. (d) The average time each animal spent per visit to area 1 and area 2 is shown for the habituation period (left plot) and after stimulus application (right plot) for premetamorphotic animals $(n=9)$ and pro/metamorphotic animals $(n=9)$. During the habituation period, no preference for either of the two areas was found. After stimulus application, both premetamorphotic animals as well as animals of higher stages were found to spend significantly more time per visit in the amino acid area. A anterior, AA amino acids, FSK forskolin, $L$ lateral, $\mathrm{M}$ medial, ON olfactory nerve, $\mathrm{P}$ posterior, PRE premetamorphosis, PRO prometamorphosis, MET metamorphosis proper, $\mathrm{vMOB}$ ventral main olfactory bulb. 
signified a change in odor evoked responses in the glomeruli of the VMOB or if the odor map remains unchanged. We loaded the ORN axons with a calcium sensitive dye and imaged changes in fluorescent signal upon stimulation with different odorant mixtures (amino acid; bile acids; amines, ATP; positive control odorant mix; negative ringer control) and forskolin (FSK), a physiological stimulant of the adenylate cyclase and the cAMP-dependent second messenger pathway.

A total of 296 glomeruli showing stimulus-induced responses were detected and analyzed in eight animals between stages 55 and 61 (Figure $3 a$ and b). For analyzes of glomerular odorant tuning profiles, we pooled glomeruli of all eight animals together. The stimulus eliciting the highest number of glomerular responses was the odorant mix (responses in $31.8 \%$ of glomeruli) followed by forskolin (26\%), amino acids (18.6\%), amines (13.5\%), bile acids (10.5\%) and ATP (9.5\%). The negative Ringer control also caused $10.1 \%$ of glomeruli to respond. Glomerular response profiles were tuned to different combinations of the applied stimuli. To understand, whether the coarse segregation into the lateral CAMP independent amino acid sensing stream and the medial cAMP-positive stream shown in tadpoles (Gliem et al., 2013) remain present during metamorphosis, we focused on the spatial distribution of glomeruli tuned to amino acids and the positive control stimulus and to all forskolin responsive profiles.

Glomeruli tuned to amino acids (plus positive control) but not activated by forskolin are located mainly in the ventro-lateral region of the $\mathrm{VMOB}$ (Figure 3a, blue). This glomerular cluster thus is not dependent on the CAMP pathway. The medial and medio-dorsal regions of the VMOB instead show a strong prevalence for glomeruli activated by forskolin and are thus CAMP-dependent (Figure 3a, yellow). Additionally, glomeruli in the dorso-lateral region were also found to be activated by forskolin (yellow asterisk, Figure 3a). A population analysis of the relative spatial position of glomeruli from lateral $(0 \%)$ to medial (100\%) shows that cAMP independent glomeruli tuned to amino acids (plus positive control) were distributed more laterally, with a relative position of $43.9 \pm 21.7 \%$ ( $n=11$ glomeruli; blue, Figure $3 b$ ), while glomeruli activated by forskolin (77glomeruli, yellow, Figure $3 \mathrm{~b}$ ) were more medially located $(62.7 \pm 19.3 \%)$.

\section{Tadpoles of different developmental stages show behavioral response to amino acids}

Our functional Calcium imaging experiments showed that the CAMP-independent signaling pathway responsive to amino acids seems to be present also during the metamorphotic innervation shift from MC to PC. Next, we performed a behavioral experiment using tadpoles of different metamorphotic stages up to metamorphotic climax to assess their behavioral response to amino acid stimulation in a choice tank (Figure 3c). Tadpoles were first left to swim freely for a two-hour habituation period and the time they spent in the two areas left (area 1, Figure 3c) and right (area 2) of the dividing wall of the tank was recorded. During the two-hour habituation period, no stimulus was applied. Tadpoles showed no inherent preference for either side of the tank (premetamorphotic tadpoles: median $13 \mathrm{~s}$ in area 1 and $13.1 \mathrm{~s}$ in area $2 ; \mathrm{n}=9$; pro/metamorphotic tadpoles: median $17.6 \mathrm{~s}$ in area 1 and $15.5 \mathrm{~s}$ in area $2 ; \mathrm{n}$ = 9; Figure 3d). 
After habituation, tadpoles were simultaneously presented with a water control and an amino acid application and the time they spent per visit in the water area (area 1) and the amino acid area (area 2) was averaged over a 20 minute recording period (Figure 3d).Tadpoles showed a preference to spend more time per visit in the amino acid area over the water control area (premetamorphotic tadpoles: median $23.4 \mathrm{~s}$ in the amino acid area, $9.5 \mathrm{~s}$ in the control area, $\mathrm{n}=9$; pro/metamorphotic tadpoles: median $77.5 \mathrm{~s}$ in the amino acid area, $15.3 \mathrm{~s}$ in the control area, $\mathrm{n}=9$; Figure $3 \mathrm{~d}$ ). Higher staged animals generally spent more time per visit in the amino acid area than lower staged animals, however tadpoles generally seem to be able to detect amino acids even during the rewiring of the vMOB clusters.

\section{Incoming ORN axons in the $\mathrm{dMOB}$ cross the midline and form a single projection area}

While in the $\mathrm{VMOB}$ new cellular components are integrated into a preexisting neuronal circuit, the $\mathrm{dMOB}$ network only starts to form during metamorphosis. We traced the ORN axons originating in the left and the right olfactory epithelium separately using two different dextran-coupled fluorophores introduced by bulk electroporation and found several differences in the network structure between the two portions of the MOB (Figure 4). Figure 4a shows representative projections of tracings from pre-, pro- and metamorphotic animals in the ventral (left images) and dorsal (right) perspective.

In the $\mathrm{VMOB}$, the axons coming in from the left and right ON project to two $\mathrm{VMOB}$ projection fields that are spatially separated at the interhemispheric midline (highlighted by the white dotted lines in the left images, Figure 4a). The general outline of the glomerular projections in the VMOB remains constant throughout metamorphosis and only slightly increases in overall volume. Contrastingly, the first axonal projections reach the $\mathrm{dMOB}$ around stage 50 (Figure 4a, top right) and project towards the interhemispheric midline. Until the onset of prometamorphosis, the incoming fibers from both ONs have formed a single projection area around the midline which grows until the end of metamorphotic development. (Figure 4a, right). During prometamorphosis and metamorphosis proper, the hemispheres of the $\mathrm{dMOB}$ projections are not clearly separated anymore, since axons cross the midline from both sides, frequently innervating glomeruli on the contralateral side. This feature of the $\mathrm{dMOB}$ becomes even more apparent when looking at a sparse cell labelling (inset, Figure 4a). Among the ORN axons crossing to the contralateral side, we found axons only connecting to the ipsi- or contralateral side, but also some axons bifurcating and innervating glomeruli on both sides of the midline.

To quantify the relative positions of incoming ORN axons from the left and right, we measured the fluorescent signal of ORN axonal projections along the left-right axis of the vMOB (Figure $4 \mathrm{~b}$, left panel) and $\mathrm{dMOB}$ (Figure $4 \mathrm{~b}$, right panel). The signals are plotted as a function of distance from the interhemispheric midline (0\%) (Figure $4 \mathrm{~b}, \mathrm{c}$ ). In the $\mathrm{VMOB}$, the distribution curves (left) clearly demonstrate symmetrical peaks in fluorescent signal on both sides of the interhemispheric midline (at approx. \pm $40 \%$ ), while in immediate proximity to the midline ( $0 \pm$ approx.10\%), no signal could be measured. This pattern did not change from earlier to later stages during metamorphosis (Figure $4 b$, left panel). In the $\mathrm{dMOB}$ on the other hand (right panel, Figure $4 \mathrm{~b}$ ), the peaks were located closer to the midline (earlier stages approx. $\pm 15 \%$ ) with some overlap around the midline (area under both curves). In the later metamorphotic stages, the two peaks almost fall onto the midline (approx. $\pm 5 \%$ ), with an even more substantial overlap. We further quantified the percentual share of innervation overlap relative to the 
total projections in animals of different stages for the $\mathrm{VMOB}$ (Figure $4 \mathrm{c}$, white dots) and $\mathrm{dMOB}$ (Figure $4 \mathrm{c}$, black dots). The average overlap of the right and left ORN axons in the vMOB amounts to $2.2 \pm 3.7 \%$ ( $n=11$, Figure $4 c$ ). The percentage of overlap in the $\mathrm{dMOB}$ increases linearly throughout metamorphosis (premetamorphosis: $9.7 \pm 7.4 \%, n=5$; prometamorphosis: $32.1 \pm 13.5 \%, n=6$; metamorphosis proper: $40.5 \pm 11.6 \%, n=5$; Figure $4 c$ ). In addition, our results show that a single glomerular structure in the $\mathrm{dMOB}$ can be composed of axon terminals from both sides (magenta and cyan arrowheads, Figure 4d). Since glomeruli are the sites of synaptic connection to postsynaptic bulbar neurons, the left/right overlap could be the basis of a bilateral signal integration system of the dMOB.

\section{Projection neuron population of the $\mathrm{VMOB}$ and the $\mathrm{dMOB}$ have different morphologies}

The connection between peripheral sensory neurons and the glomeruli differs between the VMOB and the $\mathrm{dMOB}$. To understand how these projections from the periphery connect to the postsynaptic cells, we labelled and reconstructed single projection neurons in the $\mathrm{VMOB}$ and $\mathrm{AMOB}$ of postmetamorphotic Xenopus laevis (stage 66; Figure 5).

Projection neurons in the $\operatorname{VMOB}(n=18$, Figure $5 a, c)$ and dMOB $(n=16$, Figure $5 b, d)$ share some general morphological features. Both groups have one or multiple primary dendrites originating directly from the soma (white asterisks in Figure 5) and terminating in highly branched dendritic tufts connecting to one or multiple glomeruli (insets in Figure $5 \mathrm{a}, \mathrm{b}$ ). A representative projection neuron with a single tuft in the right $\mathrm{VMOB}$ is shown in Figure $5 \mathrm{a}$ and a multi-tufted neuron in the $\mathrm{dMOB}$ in Figure $5 \mathrm{~b}$. While the tufts of neurons in the VMOB get synaptic input only from ipsilateral ORN projections (cyan projections in Figure $5 \mathrm{a}$ ) the tufts of a single projection neuron in the $\mathrm{dMOB}$ often connect to glomeruli receiving ipsi- and contralateral ORN input (cyan and magenta in Figure 5b). In addition to the tufted dendrites, most cells also have multiple dendritic branches that are not connected to the glomeruli and terminate in blunt endings (Figure $5 a, b, c ; d$, white arrowheads). The number of blunt ending dendritic branches is not different between the $\mathrm{VMOB}$ and $\mathrm{dMOB}$ population ( $8.3 \pm 2.9$ and $10.8 \pm 4.3$ respectively). Figures $5 \mathrm{c}$ and $5 \mathrm{~d}$ show reconstructions of representative projection neurons in the $\mathrm{VMOB}$ and $\mathrm{dMOB}$ that connect to a different number of glomeruli (white dotted circles).

The two populations seem to be different regarding the number of dendritic tufts and thus the number of different glomeruli that the cells get input from (grey bars in Figure 5e, left). 55.6\% of neurons labelled in the $\mathrm{VMOB}$ are uni-tufted, while only a single uni-tufted cell has been found in the $\mathrm{dMOB}$ (6.2\%). The bi- and tri-tufted neurons both make up $22.2 \%$ of the vMOB population (yellow bars in Figure $5 \mathrm{e}$, left). In the dMOB, the projection neurons maximally had five tufts, the biggest share of the population were bi-tufted and tri-tufted cells with $50 \%$ and $31.1 \%$, respectively (orange bars in Figure $5 e$, left plot). The cell populations could also be distinguished based on distance measurements: the soma-tuft distance along the dendrites (averaged for the cells with multiple tufts) was significantly longer in the dMOB cells $(322.8 \pm 96.3 \mu \mathrm{m})$ compared to the VMOB cells $(230.4 \pm 70.2 \mu \mathrm{m} ; \mathrm{p}<0.001$; Figure 5e). Similarly, the Euclidean distance between the tufts of multi-tufted cells was much longer in the dorsally located cells (vMOB $62.4 \pm 31.8 \mu \mathrm{m}, \mathrm{n}=8$; dMOB $177.6 \pm 73.4 \mu \mathrm{m}, \mathrm{n}=15 ; \mathrm{p}<0.001$; Figure 
5e). Contrastingly, the estimated tuft size was bigger in the vMOB neurons $\left(5464.2 \pm 7079.8 \mu \mathrm{m}^{3}, \mathrm{n}=\right.$ 30) than the dMOB cells $\left(1521.3 \pm 3857.7 \mu m^{3}, n=42 ; p<0.001\right)$.

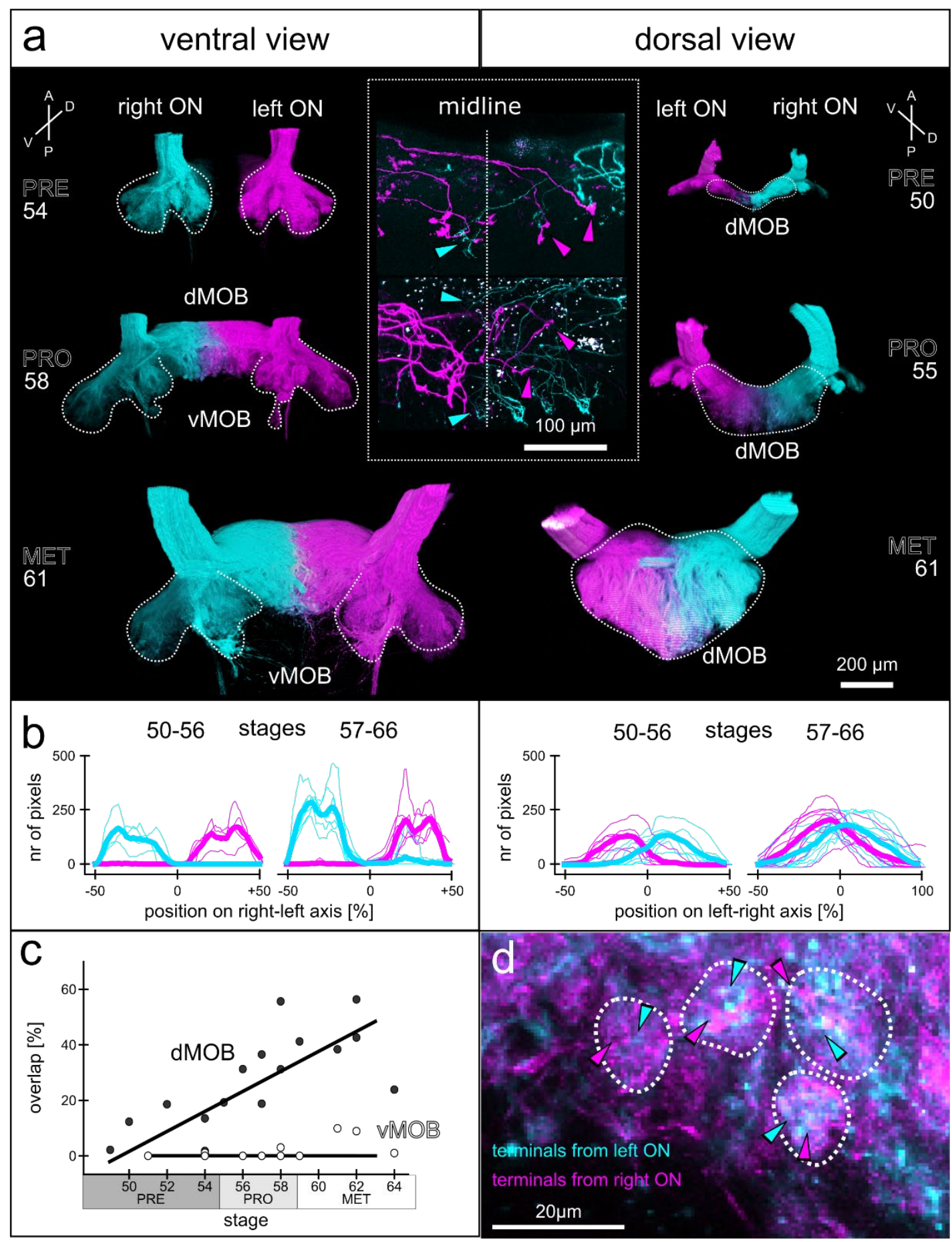

Figure 4 Innervation overlap between ORN axons from the left and right olfactory epithelia. (a) Images of ORN projections in the $\mathrm{MMOB}$ (left images, ventral view) and the $\mathrm{AMOB}$ (right images, dorsal view) representative for pre- pro- and metamorphotic animals. Projections from the left (magenta) and right (cyan) sensory epithelia were traced with two different fluorophore-coupled dextran dyes (Alexa 488 and 594 dextran) via 
electroporation. White dotted lines indicate the outlines of glomerular projections. While the left and right projections in the $\mathrm{VMOB}$ are clearly separated, the $\mathrm{AMOB}$ progressively fuses around the midline. The insert in the middle shows a closeup of sparsely labelled ORN axons crossing the midline (vertical dotted line) innervating contra- and ipsilateral glomeruli in the dMOB. (b) The fluorescent signal (in nr. of pixels) of incoming ORN projections from the left (magenta) and right (cyan) was measured along the left-right axis from $-50 \%$ to $+50 \%$, with $0 \%$ being the inter-hemispheric midline. In the $\mathrm{VMOB}$ (left plots), no fluorescent signal was detected around the midline, while in the $\mathrm{dMOB}$ (right plots) the peaks of the distributions are closer to the midline and the curves are overlapping. (c) The overlapping volume of axonal projections from the left and the right relative to the entire projection value was calculated for tadpoles of different developmental stages and separately for the vMOB (white dots) and the $\mathrm{AMOB}$ (black dots). While the $\mathrm{VMOB}$ projections have no overlapping volume, the innervation overlap in the $\mathrm{dMOB}$ increases during metamorphosis. A RANSAC regression line was fitted through the data. (d) A closeup of the projections around the midline in the $\mathrm{dMOB}$ shows, that single glomerular structures get input from both left and right olfactory epithelia (indicated by the magenta and cyan arrowheads, respectively). A anterior, D dorsal, dMOB dorsal main olfactory bulb, MET metamorphosis proper, ON olfactory nerve, P posterior, PRE premetamorphosis, PRO prometamorphosis, $\mathrm{V}$ ventral, $\mathrm{VMOB}$ ventral main olfactory bulb.

In summary, the ventral and dorsal portions of the MOB in Xenopus laevis show quite distinct properties both on the morphological as well as the circuit level. While the already existing vMOB projection fields in the premetamorphotic tadpole are gradually replaced by incoming axons from ORNs residing in the newly forming $\mathrm{MC}$ epithelium, the new $\mathrm{dMOB}$ projections form an unpaired, bilaterally innervated projection field around the midline. The overlap of the ORN axons coming in from the left and right PC increases until the end of development and single glomeruli also receive input from both sides. The postsynaptic projection neurons differ in their morphology and connectivity to the glomeruli. Around half of the ventral projection neuron population only received direct synaptic input from one glomerulus, while most projection neurons in the $\mathrm{dMOB}$ are multi-tufted.

\section{Discussion}

\section{ORN projections from the PC and MC form an aerial- and aquatic-system during metamorphosis}

During metamorphosis, the main olfactory system of most amphibians must undergo a complete transformation to adapt to the terrestrial lifestyle of the adult frog. In the case of Xenopus, the unimodal aquatic main olfactory epithelium of the tadpole transforms into a bi-modal system consisting of the water-smelling MC epithelium and the PC epithelium dedicated to aerial olfaction. This is supported by evidence showing that the larval PC and the adult MC both possess ciliated and microvillous ORNs (Hansen et al., 1998) and a similar set of olfactory receptors expressed (Syed et al., 2013,2017 ) that are tuned to detect waterborne odorants like amino acids (Syed et al., 2017). The postmetamorphotic PC on the other hand putatively expressed receptor genes more closely related to the mammalian receptors responsive to volatile odors (Freitag et al., 1995, 1998). 

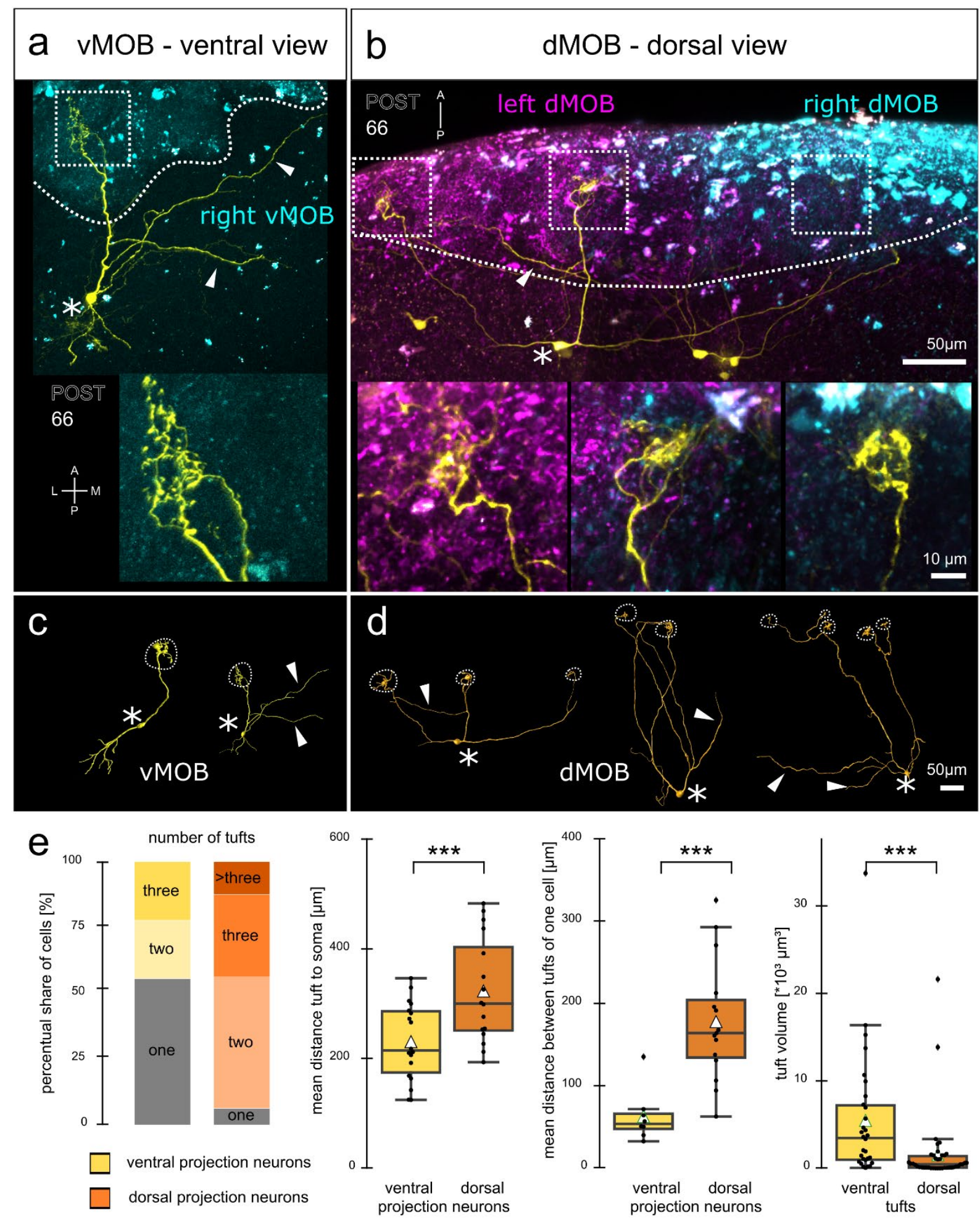

Figure 5 Different morphology of projection neurons in the $\mathrm{VMOB}$ and $\mathrm{dMOB}$ of postmetamorphotic Xenopus. ORN projections from the sensory epithelia were traced via electroporation of dextran-coupled fluorophores (left: Cascade Blue, magenta; right: Alexa 594 dextran, cyan), and projection neurons (yellow) with Alexa 488 dextran via sparse cell electroporation in the $\mathrm{VMOB}$ (a) and $\mathrm{AMOB}(\mathbf{b})$. White dotted lines indicate the ORN 
projections and the tufts (white dotted squares) are shown in a higher magnification underneath. Reconstructions of representative neurons in the $\mathrm{VMOB}$ (c) and $\mathrm{dMOB}$ (d) are shown. Asterisks indicate the projection neuron somata, white arrowheads the lateral dendritic branches without tufted terminals. (e) The bar plots on the left depict the percentual share of uni-tufted (grey) and multi-tufted projection neurons (yellow, vMOB; orange, dMOB). A higher number of uni-tufted projection neurons was found in the vMOB, while $>90 \%$ of neurons in the $\mathrm{dMOB}$ ended in at least two tufts, maximally in five tufts. Left boxplots: The distances between somata and tufts were measured along the dendritic branches for each cell (vMOB $n=18 ; d M O B n=16)$ and averaged for multi-tufted cells. The soma-tuft distance is significantly longer in cells of the dMOB. Middle boxplot: The Euclidean distance between the tufts were measured and averaged for all multi-tufted cells (vMOB $n=8 ; d M O B n=15)$. Tufts of cells in the $d M O B$ were further apart from each other. Right boxplot: Estimated tuft volumes of cells in the vMOB $(n=30)$ were significantly bigger than in the $d M O B(n=42) . * * p<0.001$. A anterior, $\mathrm{dMOB}$ dorsal main olfactory bulb, L lateral, $\mathrm{M}$ medial, ON olfactory nerve, $\mathrm{P}$ posterior, POST postmetamorphosis, vMOB ventral main olfactory bulb.

The projections of the ORNs in adult frogs towards their glomerular targets in the MOB have been described based on their lectin binding pattern (Franceschini et al., 1992; Hofmann and Meyer, 1991; Key and Giorgi, 1986). Fibers coming from the adult MC were found to be soybean-agglutinin positive and innervate the ventro-lateral $\mathrm{MOB}$, while the 'aerial-fibers' from the PC were soybean-agglutinin negative and projected into the dorso-medial MOB (Gaudin and Gascuel, 2005; Hofmann and Meyer, 1991). In a thorough study of ORN projection fields in the MOB during development and metamorphosis, Gaudin and Gascuel describe, that the dMOB (called PF9 in their study) increases significantly in size ( 28 times increase) between stage 50 and 59, while the increase factor dropped to 1.4 in stages between 59 and 64 (Gaudin and Gascuel, 2005). Our results confirm these findings (Figure 1). We did not evaluate the absolute growth of the olfactory bulb structures, but the changes in percentual share of the projection zones in the $\mathrm{VMOB}$ and the $\mathrm{dMOB}$. We found that the biggest increase in the relative size of the $\mathrm{dMOB}$ happened until stages $55 / 56$ with an increase from $0 \%$ to around $60 \%$ of the total volume, while the increase was only $10 \%$ from stages 57 to 64 (Figure 1c). We additionally found that around stage 54 , the projection fields of the $\mathrm{dMOB}$ and the $\mathrm{VMOB}$ already have approximately the same volume.

The $\mathrm{dMOB}$ only gets input from cells residing in the PC from the start of its formation around stage 49/50 (Burd, 2000; Reiss and Burd, 1997a). We investigated incoming fibers to the dMOB in animals throughout metamorphosis and found that input into the $\mathrm{AMOB}$ was solely originating from the PC. We did not find any fibers from the MC (Figure $2 b$, right bar plot). The VMOB of the tadpoles is innervated by ORN axons from the PC. Around stage 52 , the first axons from the newly formed MC reach the $V M O B$ glomeruli (Burd, 2000; Reiss and Burd, 1997a). Reiss and Burd observed, that from stages 52 to $58, P C$ axons could still be observed in the VMOB, while after that stage, the PC afferents have completely vanished from the $\mathrm{VMOB}$, leaving only the MC axon terminals (Reiss and Burd, 1997a). We have quantified this dynamic shift in innervation in the $\mathrm{VMOB}$ and found that the relative innervation of the PC decreases gradually from stage 52 to around stage 61 (Figure $2 b$, left plot). We still observed some PC axons after the onset of metamorphotic climax (stage 58), even if by that time in the development, $\mathrm{VMOB}$ glomeruli are already majorly innervated by incoming MC ORN axons. Our 
data also is in accordance with results showing a peak in cellular apoptosis in the PC around stage 58 and later again around 62 (Dittrich et al., 2016). Some ORNs in the PC projecting to the VMOB could still be present at stages 58-62, undergoing apoptosis during the second apoptotic peak around stage 62, later than proposed by Reiss and Burd (Reiss and Burd, 1997a).

\section{Dynamic innervation shift of the vMOB does not disrupt glomerular or behavioral responses to odorants}

During the described rewiring process in the $\mathrm{VMOB}$, it is unclear so far whether the glomeruli retain their larval functionality, if the odorant response pattern is reorganized or if they temporarily loose a clear functional pattern. For the first time, we imaged odorant induced response profiles of glomeruli in tadpoles up to stage 61 in the 'water bulb' of Xenopus laevis and found that the extensive fiber replacement and reorganization show little effect on the functional responsiveness in the VMOB. Odorant mediated responses can be recorded in the glomeruli of the vMOB up until the metamorphotic climax (Figure 3a, b). This suggests that the replacement of fibers happens gradually, to ensure that the animals do not lose odorant sensitivity during metamorphosis. By the time the PC fibers innervating the $\mathrm{VMOB}$ have all undergone apoptosis, the new connections from the $\mathrm{MC}$ seem to have functionally replaced them. The behavioral assay supports this idea exemplarily for amino acid sensitivity (Figure 3c). Even though it is unclear, which ecological function amino acids have for Xenopus tadpoles, they seem to prefer amino acids over the negative control stimulus up until the metamorphotic climax.

Odorant coding in the MOE and the $\mathrm{MOB}$ of Xenopus laevis tadpoles have been extensively studied (Gliem et al., 2013; Manzini and Schild, 2003, 2010; Syed et al., 2017), but little to no functional imaging data exists for the metamorphotic and postmetamorphotic stages. In a comprehensive study from the MOE to the MOB of tadpoles, it was shown that the MOB projections can be subdivided into two parallel processing streams (Gliem et al., 2013). Microvillous ORNs projecting to the lateral glomeruli have a cAMP-independent second messenger pathway (Manzini and Schild, 2003; Manzini et al., 2002) and are mostly responsive to amino acids (Gliem et al., 2013). The medial stream relies on the canonical cAMP-dependent pathway instead and is formed by ciliated ORNs (Gliem et al., 2013). It was hypothesized that the lateral stream could be linked to ORNs expressing vomeronasal type 2 olfactory receptors (V2Rs) expressed in the lateral MOE of the larvae (Gliem et al., 2013; Syed et al., 2013, 2017). The expression pattern of the V2Rs and the response pattern to amino acids gradually shifts to the adult MC during metamorphosis (Syed et al., 2017). We show that the segregation between the lateral amino-acid sensitive, non-CAMP pathway and the CAMP dependent medial pathway is retained until stage 61 tadpoles (Figure 3). The difference maps in Figure 3a clearly show, that forskolin-induced calcium transients (yellow) are missing in the lateral glomerular cluster, while responses to amino acids are present in these glomeruli (blue). A new dorso-lateral cluster of cAMP-dependent glomeruli has been identified in our study (yellow asterisks, Figure 3a), which differs from the other ventro-lateral glomeruli. A complete odor map of both the larval and adult $\mathrm{VMOB}$ however is still not available. We can thus not completely rule out, that the odorant map changes in its exact topography during metamorphosis. However, the metamorphotic conservation of the lateral amino acid-sensitive stream 
suggests a stable odorant map in the $\mathrm{VMOB}$ at least on a coarse level. Odorant receptor molecules themselves are thought to be a main determinant factor for ORN axonal targeting in the MOB in vertebrates (Feinstein and Mombaerts, 2004; Mombaerts, 2006). If the MC indeed expresses the same olfactory receptors as the larval PC epithelium (Syed et al., 2017), it seems likely that the vMOB odor map also remains constant.

Axon targeting mechanisms in the $\mathrm{VMOB}$ of Xenopus are likely to differ from the targeting in the $\mathrm{dMOB}$, since their two hemispheres do not present separate anatomical entities. Up to date, no odorant map of the dorsal projections is available. To our knowledge, it is also unclear whether the dMOB of anurans is functionally organized symmetrically or whether ORN axons expressing a given receptor in both MOEs project their axons to a single glomerulus.

\section{Putative different origin of the OB fusion in anurans and birds}

The fused olfactory bulb is not a feature exclusive to the anurans, it has also been described in some fishes and some bird species (Nieuwenhuys, 1966). It has been described in more detail in passeriform birds (Carl Huber and Crosby, 1929; Corfield et al., 2015; Yokosuka et al., 2009b, 2009a). However, in birds the fusion on the gross anatomical level does not seem to relate to a functional overlap. In the Japanese Jungle Crow, it was shown that even though the olfactory bulb appears as one single mass, the ONs project separately to the left and right glomerular layer, with no significant left-right overlap (Yokosuka et al., 2009a). In contrast, the morphological results in the present study (Figure 4) as well as electrophysiological studies in ranid frogs (Jiang and Holley, 1992b) show a significant overlap between the ORN axon projection.

It is generally assumed that the relative size of the olfactory bulbs is correlated with the importance of olfactory capability across vertebrates (Corfield et al., 2015; Yopak et al., 2015). Among birds, the Passeriformes only possess a very small olfactory bulb in relation to the whole brain size (Corfield et al., 2015), suggesting a relative loss of reliance on their sense of smell. This is partially explained by the fact that these birds, especially the corvids, have evolved higher cognitive functions (Corfield et al., 2015; Emery, 2006). Their small, fused olfactory bulb could thus be sign of evolutionary degeneration of olfactory function. In anurans, the fused olfactory bulb has been described in different species (Ebbesson et al., 1986; Hoffman, 1963; Jiang and Holley, 1992b; Scalia et al., 1991a), independently of their habitat and the degrees to which they rely on olfaction. It seems thus unlikely that the fusion of the olfactory bulb can be interpreted as a sign of olfactory degeneration in the anurans as well.

The evolutionary absence of the fused olfactory bulb in most vertebrates suggests, that it is a derived evolutionary feature in both birds and frogs rather than an ancestral trait. Also, the close sister clade of the anurans, the salamanders, have two separated olfactory bulbs. We thus propose a different origin of this feature and a putatively different function in the various animal groups.

\section{Projection neurons in the $\mathrm{VMOB}$ and the $\mathrm{dMOB}$ are morphologically different}

The morphology of the primary projection neurons in the olfactory bulb has been extensively studied in a variety of vertebrates (for review see Dryer \& Graziadei, 1994; Nieuwenhuys, 1966). Mammalian 
projection neurons are divided into mitral and tufted cells with further subtypes being distinguished (Nagayama et al., 2014). Mitral cell somata are generally bigger in size and located more caudally, tufted cells are smaller and their somata are located closer to the glomerular layer (Dryer and Graziadei, 1994; Macrides et al., 1985; Mori et al., 1983; Nagayama et al., 2014). In amphibians, it is still debated whether the population of projection neurons in the MOB can be subdivided (Herrick, 1924; Jiang and Holley, 1992a; Laberge, 2008; Scalia et al., 1991a). Morphological (Jiang and Holley, 1992a; Scalia et al., 1991a) and electrophysiological studies (Jiang and Holley, 1992b) in adult ranid frogs propose a similar subdivision into tufted-like and mitral-like projection neurons in anurans as well.

In the present study we tackled the question whether the projection neuron populations in the VMOB (water system) and the $\mathrm{dMOB}$ (air system) of the aquatic Xenopus are morphologically different. It is described for amphibians, reptiles and fish (Dryer and Graziadei, 1994), that projection neurons possess multiple primary dendrites that terminate in glomerular tufts and a variable number of lateral dendrites without tufts (Dryer and Graziadei, 1994; Nieuwenhuys, 1966; Scalia et al., 1991a). This is in accordance with our results, no difference could be found between cells in the $\mathrm{VMOB}$ and $\mathrm{dMOB}$ regarding these aspects. Because of the difficult distinction between primary and secondary dendrites in amphibians, we measured the blunt endings of the neuronal structure as an estimate for non-tufted secondary dendrites. We found around 8 blunt ending dendrites in the VMOB cells and 10 in the $\mathrm{dMOB}$ cells. These numbers are a higher estimate to the mostly 2-3 secondary dendrites counted in ranid frogs (1-6) (Jiang and Holley, 1992a). This difference could derive from the fact that secondary dendrites bifurcate two to three times before reaching their blunt endings. The data are also comparable to that of other animals like salamander (0-5; Laberge, 2008), rabbit (2-5; Mori et al., 1983), rat (2-9; Dryer \& Graziadei, 1994; Orona et al., 1984) and hamster (1.7-4.7; Macrides \& Schneider, 1982).

While mammalian mitral/tufted cells in the MOB possess a single primary dendrite connecting to a single glomerulus, projection neurons of earlier diverging vertebrates often innervate multiple glomeruli (Dryer and Graziadei, 1994; Nieuwenhuys, 1966). In the population analysis of projection neurons in the ranid frogs, around $50 \%$ of examined neurons were found to be connected to a single glomerulus, $30 \%$ were bi-glomerular and $20 \%$ innervated more than two glomeruli (Jiang and Holley, 1992a). This distribution is quite close to the counts we obtained in the vMOB (56\% uni-, $22 \%$ bi-, $22 \%$ tri-glomerular: Figure 5e). Interestingly, the number of glomeruli innervated by a single ORN axon in anurans also follows a similar distribution across different species and developmental stages (tadpoles $41 \%$ uni-glomerular, 59\% multi-glomerular; juveniles: 50\% uni, 50\% multi-glomerular; Weiss et al., 2020). It is still unclear if there could be a functional correlation between the different types of these distributions.

Contrastingly, In the dMOB we could only find one exceptional uni-glomerular projection neuron, which had a single dendritic tuft significantly bigger than the remaining ones (Figure 5e). It is perceivable that this tuft could connect to many adjacent glomeruli. 50\% of the dMOB projection neurons were classified as bi-glomerular and the remaining neurons connected to up to five distinct 
glomeruli (Figure 5). In addition to the apparent prevalence of the multi-glomerular pattern over the uni-glomerular one, the dendritic tuft volumes in the $\mathrm{AMOB}$ were significantly smaller than in the VMOB (Figure 5e). This trend could also be reflected in a small difference in glomerular diameter for ventrally $(\sim 27 \mu \mathrm{m})$ and dorsally located glomeruli ( $24 \mu \mathrm{m})$, even though the general sizes of glomeruli vary quite extensively (Gaudin and Gascuel, 2005; Nezlin and Schild, 2000).

Projection neurons in the $\mathrm{AMOB}$ also displayed a larger distance between glomeruli compared to the vMOB cells (Figure 6B, E). It has been shown in different animals and olfactory subsystems, that peripheral olfactory input seems to segregate into functional clusters within the glomerular array (Braubach et al., 2012; Gliem et al., 2013; Green et al., 2017; Wagner et al., 2006). A larger distance between the dendritic tufts could thus be a sign that single projection neurons integrate between different odorant classes. Reversely, since the $\mathrm{dMOB}$ is an unpaired structure consisting of ORN axons from the left and the right olfactory epithelia, the tufts could be integrating the signal from the two homologous glomeruli of the right and left side. In that case, the cells would integrate the same odor information coming from the two epithelia. Either way, the multi-glomerular pattern in the dMOB could imply an integrative function, that seems more important for aerial smell than for aquatic smell of the frogs.

\section{Putative ecological and behavioral relevance of the dMOB}

The presence of a well-developed system to sample airborne odorants seems counterintuitive in a fully aquatic frog. It has however been reported that Xenopus move overland mostly in search for other water bodies or food (Measey, 2016; Du Plessis, 1966). It has also been suggested, that olfactory cues might play a role in overland orientation and migrations (Savage, 1965). In the present study, we show how the neuronal circuit of the dorsal 'air-bulb' in Xenopus differs from the ventral 'water bulb' and most other vertebrate MOBs. The bilateral innervation of both ORNs and projection neurons gives it a high degree of integrative power. The importance of integration of bilateral sensory information has been shown for other sensory systems, but its role in olfaction has only been shown in rare instances (Catania, 2013). Among anurans, the fusion of the two MOBs seems to be an ancestral trait with so far unknown function (Ebbesson et al., 1986; Eisthen and Polese, 2007; Scalia et al., 1991a). The anuran $\mathrm{dMOB}$ could be a primary olfactory center integrating odorant information from the left and right nose in single, bilaterally innervated glomeruli or by the multiple tufts of single projection neurons. This could e.g. facilitate the overland spatial orientation of Xenopus. Since little is known about the use of odorant cues in frogs in general, it is intriguing to think that the special input integration in the dMOB could be part of a spatial integration center used in orientation behavior. 


\section{Acknowledgements}

We thank the members of the Manzini Lab for fruitful discussion. This work was supported by DFG Grant 4113/4-1.

\section{Conflict of Interest}

The authors declare that they have no competing interests.

\section{Author Contributions}

L.W., T.H., and I.M. conceptualized the study. L.W., P.S.A, S.J.H. investigation; L.W., T.O., S.J.H. formal analysis, visualization L.W. writing of the original draft; L.W., T.O., S.J.H., T.H., and I.M. writing-review and editing the manuscript; T.H. and I.M. funding acquisition and resources of the manuscript supervision of the article.

\section{Ethics statement}

All experiments performed followed the guidelines of Laboratory Animal Research of the Institutional Care and Use Committee of the Justus-Liebig-University Giessen (GI 15/7, 932_GP).

\section{Funding}

This work was supported by DFG Grant 4113/4-1. 


\section{Chapter 5: Multi-glomerular projection of single olfactory receptor neurons is conserved among amphibians}

Published as Original Research article in the Journal of Comparative Neurology and available under https://doi.org/10.1002/cne.24887

\section{Authors and affiliations}

Lukas Weiss ${ }^{1,5}{ }^{*}$, Lucas D. Jungblut ${ }^{2}$, Andrea G. Pozzi ${ }^{2}$, Barbara S. Zielinski ${ }^{3}$, Lauren A. O'Connell ${ }^{4}$, Thomas Hassenklöver ${ }^{1}$ and Ivan Manzini ${ }^{1}$

1 Department of Animal Physiology and Molecular Biomedicine, University of Giessen, 35392 Giessen, Germany

2 Departamento de Biodiversidad y Biología Experimental, IBBEA-CONICET, Universidad de Buenos Aires, C1428EGA Buenos Aires, Argentina

3 Department of Integrative Biology, University of Windsor, N9B 3P4 Windsor, Ontario, Canada

4 Department of Biology, Stanford University, 94305 Stanford, California, USA

5 Lead Contact

* Correspondence: lukas.weiss@physzool.bio.uni-giessen.de (L.W.)

\section{Contribution statement}

I conducted all experiments and analyses, arranged the figures and wrote the manuscript. I conceptualized the study together with T.H. and I.M. L.D.J, A.G.P, B.S.Z. and L.A.O provided lab-space, animals and materials. All authors were involved in reviewing and editing the manuscript.

\section{Keywords}

olfaction, glomeruli, axonal wiring, evolution, sensory system, fish, frog, salamander 


\begin{abstract}
Individual receptor neurons in the peripheral olfactory organ extend long axons into the olfactory bulb forming synapses with projection neurons in spherical neuropil regions, called glomeruli. Generally, odor map formation and odor processing in all vertebrates is based on the assumption that receptor neuron axons exclusively connect to a single glomerulus without any axonal branching. We comparatively tested this hypothesis in multiple fish and amphibian species (both sexes) by applying sparse cell electroporation to trace single olfactory receptor neuron axons. Sea lamprey (jawless fish) and zebrafish (bony fish) support the unbranched axon concept, with $94 \%$ of axons terminating in single glomeruli. Contrastingly, axonal projections of the axolotl (salamander) branch extensively before entering up to six distinct glomeruli. Receptor neuron axons labeled in frog species (Pipidae, Bufonidae, Hylidae and Dendrobatidae) predominantly bifurcate before entering a glomerulus and $59 \%$ and $50 \%$ connect to multiple glomeruli in larval and post-metamorphotic animals, respectively. Independent of developmental stage, lifestyle and adaptations to specific habitats, it seems to be a common feature of amphibian olfactory receptor neuron axons to frequently bifurcate and connect to multiple glomeruli. Our study challenges the unbranched axon concept as a universal vertebrate feature, and it is conceivable that also later diverging vertebrates deviate from it. We propose that this unusual wiring logic evolved around the divergence of the terrestrial tetrapod lineage from its aquatic ancestors and could be the basis of an alternative way of odor processing.
\end{abstract}

\title{
Graphical abstract
}

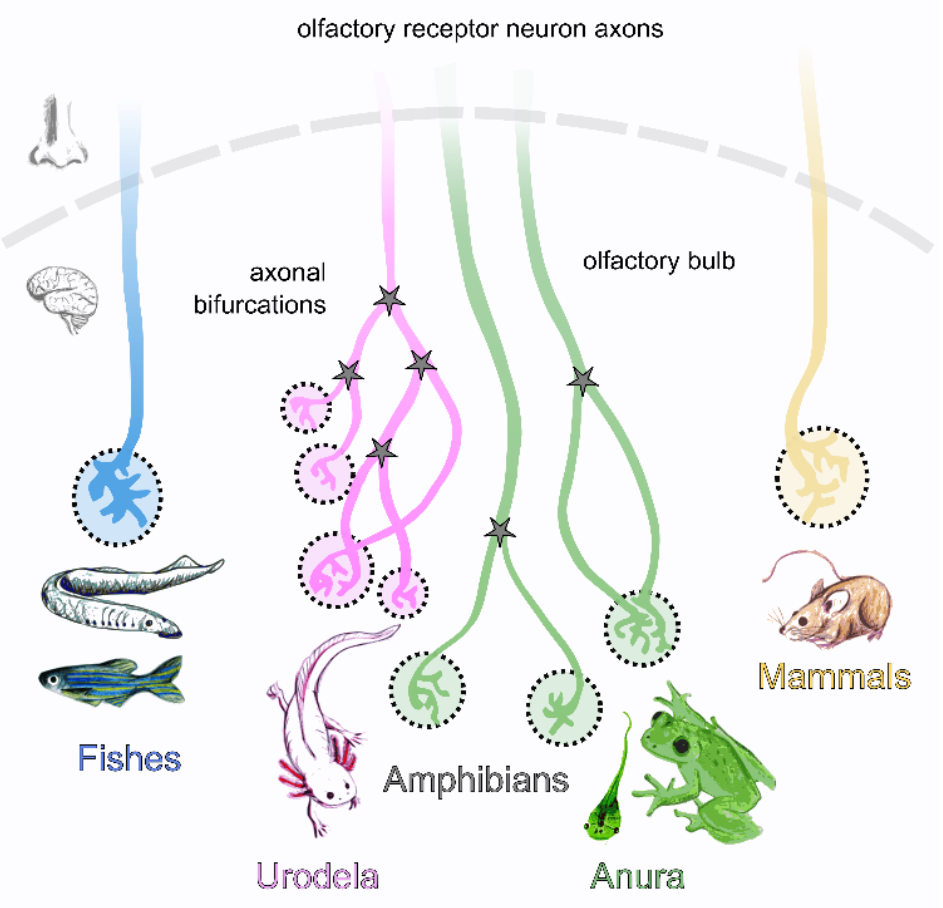




\section{Introduction}

Vertebrates are equipped with a sophisticated olfactory system to detect relevant chemical information about their environment. Throughout vertebrate evolution, a progressive segregation into parallel olfactory pathways takes place (Bear et al., 2016; Eisthen, 1997; Munger et al., 2009). While the peripheral olfactory organ of fishes consists of a single olfactory surface (Hamdani and Døving, 2007; Hansen and Zeiske, 1998; Sato, 2005; but see Ren et al., 2009) the mammalian system is segregated into several anatomically and functionally distinct subsystems (Breer et al., 2006; Munger et al., 2009). A first bipartition of the olfactory periphery into a main olfactory epithelium (MOE) and a vomeronasal organ (VNO) coincides with the evolution of the first tetrapods, the amphibians (Eisthen, 1992). However, primordial structures that could potentially be homologous to the VNO have been identified in earlier diverging vertebrates like lungfish (González, 2010) and lamprey (Ren et al., 2009).

Chemical detection in the various sensory epithelia is relying on the expression of olfactory receptor proteins in the dendritic cilia or microvilli of olfactory receptor neurons (ORNs) (Mombaerts, 1999), with the two major receptor gene families being the OR-type olfactory receptor genes and the vomeronasal receptor genes (Buck and Axel, 1991; Dulac and Axel, 1995). In the main olfactory system of rodents, each ORN expresses a single OR-type olfactory receptor (Chess et al., 1994; Malnic et al., 1999) and sends a single, unbranched axon to the olfactory bulb (OB) via the olfactory nerve (ON). In the main $\mathrm{OB}$, an axon terminally branches in the confines of a single dense neuropil structure, a glomerulus. All axons of ORNs equipped with the same olfactory receptor type coalesce onto one or very few glomeruli (Mombaerts, 2006; Ressler et al., 1994; Vassar et al., 1994). Each glomerulus in the main $O B$ is thus believed to relay the information of a single OR-type olfactory receptor to the postsynaptic projection neurons, distinguished as mitral and tufted cells in rodents (Bear et al., 2016)This constitutes the idea of the chemotopic organization of the rodent main OB (Mombaerts et al., 1996). While mammalian projection neurons extend their single primary dendrite into one sole glomerulus, projection neurons in fish, amphibians and reptiles often bear several primary dendrites connecting to multiple glomeruli (for review see Dryer and Graziadei, 1994).

In contrast to the wiring logic employed by the main olfactory system, all vomeronasal receptor neurons (VRN) in the VNO expressing the same type of vomeronasal receptor converge onto 15-30 glomeruli in the mammalian accessory olfactory bulb (AOB) (Belluscio et al., 1999; Rodriguez et al., 1999). Projection neurons in the $A O B$ of many animals have long been known to extend multiple dendrites into spatially distinct glomeruli (Ramón y Cajal, 1911). However, it is still under debate whether the synaptic input that a single projection neuron in the $\mathrm{AOB}$ receives from multiple glomeruli contains the information conveyed by different or by the same type of vomeronasal receptor (Del Punta et al., 2002b; Wagner et al., 2006).

The basic olfactory wiring principles have long been assumed to be uniform among vertebrates. The first vertebrate species that has been found to violate the rule of an unbranched ORN axon innervating a single glomerulus in the main OB was the African clawed frog Xenopus laevis (Nezlin and Schild, 
2005). The majority of examined axons were shown to be connecting to more than one glomerulus in larval animals (Nezlin and Schild, 2005) and this alternative pattern was retained after metamorphosis (Hassenklöver and Manzini, 2013). It remains elusive whether this multi-glomerular wiring is a specific adaptation of the secondarily aquatic Xenopus or if it is a more conserved evolutionary feature also present in other vertebrate lineages.

Here, we report that bifurcating ORN axons and multi-glomerular innervation are not a particular adaptation of Xenopus laevis, but a conserved feature throughout the order Anura (frogs and toads). ORN axon tracings in four ecologically diverse frog species in pre- and post-metamorphotic animals showed that this alternative olfactory wiring scheme is independent of developmental stage and of habitat. We could also show that multi-glomerular innervation of single ORN axons is the predominant pattern in the axolotl salamander, which suggests that this feature might be present in all amphibians. Contrastingly, both the main olfactory system of the sea lamprey (jawless fish) as well as the olfactory system of zebrafish (teleost fish) follow the unbranched ORN axon paradigm with a single ORN axon only arborizing within the confines of a single glomerulus. We propose that the unusual wiring logic found in amphibians evolved around the divergence of the terrestrial tetrapod lineage from its aquatic ancestors and forms the basis of an alternative way of odor processing.

\section{Material and Methods}

\section{Animals}

\section{Fish species:}

All sea lampreys (Petromyzon marinus) used in this study were post-larval transformer stages (metamorphic stage seven, both sexes, approx. $12 \mathrm{~cm}$ in length) from the Connecticut River, Turner Falls, MA. Animals were captured and supplied by United States Geological Survey Conte Anadromous Fish Research Laboratory. They were kept in $420 \mathrm{I}$ tanks at $6^{\circ} \mathrm{C} \pm 1^{\circ} \mathrm{C}$ under static renewal conditions until used. Wild type zebrafish (Danio rerio, both sexes) were kept in oxygenated water tanks at room temperature.

\section{Amphibian species:}

Albino larvae of axolotl (Ambystoma mexicanum) were obtained from the Ambystoma Genetic Stock Center at the University of Kentucky, USA. They were kept in oxygenated water tanks $\left(20^{\circ} \mathrm{C}\right)$ and fed with red mosquito larvae. Animals used for this study were both sexes, 5-6 weeks of age.

Wild type Xenopus tropicalis larvae were bred and reared at the Institute of Animal Physiology, University of Giessen. They were kept in water tanks at a water temperature of $25^{\circ} \mathrm{C}$ and fed with algae. Animals used for this study were stages 49-52 after Niewkoop and Faber (Nieuwkoop and Faber, 1994). 
Ranitomeya imitator tadpoles of stages 28-31 after Gosner (Gosner, 1960) were bred in the laboratory colony at the Biology Department of Stanford University, Palo Alto, CA, USA. Individual tadpoles were kept separately after hatching at a water temperature of $25^{\circ} \mathrm{C}$.

Rhinella arenarum larvae (Gosner stages 29-31) and juvenile animals were obtained by in vitro fertilization from a colony at the Faculdad de Ciencias Exactas y Naturales of the University of Buenos Aires. Scinax granulatus larvae (Gosner stages 31-35) and juveniles were collected from the wild (semitemporary ponds formed in the surroundings of the Campus of the University of Buenos Aires). All larval animals were kept in tanks of dechlorinated water at a temperature of $22^{\circ} \mathrm{C}$ and fed ad libitum with chard leaves. Juvenile animals were kept in glass terraria and fed with flies. It was not distinguished between sexes.

\section{Sparse cell electroporation}

Animals were anesthetized using $0.02 \%$ MS-222 (ethyl 3-aminobenzoate methanesulfonate; SigmaAldrich) in tap water until completely unresponsive and placed on a wet tissue paper under a stereomicroscope. ORNs were stained using micropipettes pulled from borosilicate glass capillaries (Warner instruments, resistance 10-15 M $\Omega$ ) filled with fluorophore-coupled dextrans (Alexa dextran 488 and 594, $10 \mathrm{kDa}$, Life Technologies) diluted at a concentration of $3 \mathrm{mM}$ in saline Ringer (Amphibian Ringer (mM): $98 \mathrm{NaCl}, 2 \mathrm{KCl}, 1 \mathrm{CaCl}_{2}, 2 \mathrm{MgCl}_{2}, 5$ glucose, 5 Na-pyruvate, 10 Hepes, pH 7.8; Lamprey Ringer (mM): $130 \mathrm{NaCl}, 2.1 \mathrm{KCl}, 2.6 \mathrm{CaCl}_{2}, 1.8 \mathrm{MgCl}_{2}$, 4 Hepes, 4 dextrose, $1 \mathrm{NaHCO}_{3}, \mathrm{pH} 7.4$, Zebrafish Ringer (mM): $131 \mathrm{NaCl}, 2 \mathrm{KCl}, 20 \mathrm{NaHCO}_{3}, 1.25 \mathrm{KH}_{2} \mathrm{PO}_{4}, 2.5 \mathrm{CaCl}_{2}, 2 \mathrm{MgSO}_{4}, 10$ dextrose, 5 Na-pyruvate, 10 Hepes, $\mathrm{pH}$ 7.2). The pipettes were mounted on the electrode bearing headstage of an Axoporator 800A (Axon instruments, Molecular Devices) and inserted into the main nasal cavity of the animals. A $500 \mathrm{~ms}$ train of square voltage pulses $(50 \mathrm{~V}$, single pulses $300 \mu \mathrm{s}$ at $200-300 \mathrm{~Hz}$ ) was triggered to stain neurons (Haas et al., 2001; Hassenklöver and Manzini, 2013). Staining of neurons primarily depends on their proximity to the pipette tip opening. It is conceivable, that labeling success is influenced by neuronal morphology and size. Nevertheless, to the best of our knowledge, this method is suited to label all types of neurons. This protocol was repeated at different positions inside the main nasal epithelia. The animals were left to recover. We used two distinct fluorophores to label multiple axons per animal or OB-hemisphere.

\section{Olfactory bulb whole mount preparation}

Animals were anesthetized again (as described above) three days after electroporation and killed by severing the spinal cord at the level of the brainstem. The whole olfactory bulbs were dissected out of the tissue. Samples were immediately imaged in Ringer's solution or fixed in 4\% PFA in PBS for one hour and imaged later.

\section{Image acquisition and processing}

OB samples were placed under the microscope and rotated to ensure optimal visualization of labelled ORN axons. Multi-channel image stacks (z-resolution of $1 \mu \mathrm{m}$ ) were acquired using multi-photon microscopy at an excitation laser-wavelength of $780 \mathrm{~nm}$ (upright Nikon A1R-MP and upright Leica SP5 
multiphoton microscopes). Brightness and contrast of the stacks were adjusted using ImageJ (Schindelin et al., 2012). Since there was no dye introduced into the tissue with a blue-wavelength emission, we used the blue-wavelength detector ( $400-492 \mathrm{~nm}$ ) to image tissue auto-fluorescence. Pigmentation derived autofluorescence was mathematically subtracted from the other emission channels using the image-calculator function implemented in ImageJ. Image data are presented as maximum intensity projections along the z-axis of the virtual image stacks.

\section{Axonal reconstructions}

Individual neuronal morphology was reconstructed semi-automatically by defining branching and endpoints of the axonal structure from the acquired image stacks in Vaa3D (Peng et al., 2010). Only ORN axons that could be traced from the beginning of the nerve layer in the olfactory bulb until their terminals in the glomerular layer were reconstructed. The outer curvature of the OB hemisphere at the ON entry point (identified via tissue autofluorescence) was used as boundary. The reconstructed neuron-trees were sorted by the sort_swc algorithm implemented in Vaa3D to define the root of the axon as first node of the structure.

\section{Structural analysis and identification of glomeruli}

Axonal reconstructions were analyzed and quantified using custom written Python scripts in Jupyter notebook. Spatial distribution of branching points and length of branches and sub-branches were assessed based on the reconstructions. All data presented in regard to branch length is measured in distances along the axonal structure (in $\mu \mathrm{m}$ ) or displayed as length-ratio in \%.

The number of glomeruli innervated by an axonal structure was determined using the DBSCAN algorithm (Density-Based Spatial Clustering of Applications with Noise) implemented in the sklearn machine learning package written for Python (Pedregosa et al., 2011). The algorithm clusters together points that are in spatial proximity to a lot of neighboring points (glomerular cluster) while it marks points far away from its closest neighbors as low-density noise. All branching- and endpoints of a neuron-tree structure from the root to the terminals of the axons were used as input points. The algorithm is designed to identify point clusters of various, non-linearly separable shapes. Based on the algorithm, an axon terminal was considered a glomerular cluster if at least three points were in spatial proximity. Blunt axonal endings without terminal bifurcations were marked as noise outliers. To account for the different glomerular size between the various animals used for this study, the minimal distance for points to be considered a cluster was chosen separately for each species and ontogenetic stage (in $\mu \mathrm{m}$ : $P$. marinus $90, D$. rerio $20, A$. mexicanum $20, X$. tropicalis $11, R$. arenarum premetamorphotic 10 , post-metamorphotic $15, S$. granulatus pre-metamorphotic 14 , postmetamorphotic $16, R$. imitator 10$)$. These values were chosen based on previously reported glomerular size (P. marinus: Green et al., 2017; D. rerio: Braubach et al., 2012) or our own glomerular tracing experiments (all amphibian species). 


\section{Statistical Analysis}

Averaged data are presented as mean \pm standard deviation. Statistical significance was determined by Kruskal-Wallis rank sum test followed by Dunn's multiple comparison post-hoc test, unless otherwise stated. To control familywise error rate for multiple comparisons, a Holm-Bonferroni correction was applied.

\section{Results}

\section{ORN axons in fish have fewer branching points than amphibian axons}

The morphology of an ORN axon in vertebrates is generally described as an unbranched projection terminating in fine arborizations within a single glomerulus of the OB (Klenoff and Greer, 1998). It was already reported that this principle does not apply to the wiring scheme in the secondarily aquatic African clawed frog (Hassenklöver and Manzini, 2013; Nezlin and Schild, 2005). We investigated whether this alternative projection pattern could be more common in the olfactory system of other aquatic vertebrates. We traced single ORNs from the olfactory epithelium of the fully aquatic postlarval sea lamprey ( $P$. marinus, jawless fish), the zebrafish (D. rerio, bony fish), the axolotl ( $A$. mexicanum, urodela) and the larval clawed frog (X. tropicalis, anura; phylogenetic overview in Figure $1 b)$ to their axon terminals in the glomeruli of the OB. No experiments were conducted in the accessory olfactory system.

Axon tracings of the four species differed substantially in their general branching structure (Figure 1a). ORN axons in the OB of the sea lamprey and the zebrafish showed similarity with the pattern reported for rodents (Klenoff and Greer, 1998). A long, unbranched axon projects towards the glomerular layer of the $\mathrm{OB}$ where it terminally arborizes. While both species follow this common feature, the zebrafish axons have even shorter and fewer terminal arborizations than the lamprey axons. In contrast, the ORN axons of the two amphibian species bifurcate shortly after entering the OB, projecting several sub-branches into the glomerular layer, where each sub-branch arborizes again. Quantifying the bifurcations of each single axon along the distance from its entry point in the $\mathrm{OB}$ to the axon terminals, we found significant differences between fishes and amphibians (Figure $2 a$ and $b$ ). Sea lamprey axons have on average $6.5 \pm 4.5$ terminal arborizations ( $n=6$, five animals). This pattern is not significantly different from the zebrafish axons that show even fewer arborizations ( $3 \pm 1.4, n=10$, ten animals). Zebrafish axons were found to have the least complex structure, with the highest amount of arborizations for a single axon being five. The axolotl displays a significantly different pattern from both the lamprey and zebrafish axons ( $p=0.0058$ and 0.00001 respectively, Figure $2 b$ ), the ten examined axons (seven animals) bifurcate $29.7 \pm 7.7$ times on average, with one axon even branching 42 times. Axonal tracings obtained from the tadpoles of the clawed frog ( $23 \pm 8.1, n=10$, eight animals) also showed a significantly higher degree of branching when compared to the zebrafish $(p=0.0013)$. They did not show significantly different branching points than lamprey $(p=0.08)$ or axolotl axons. 


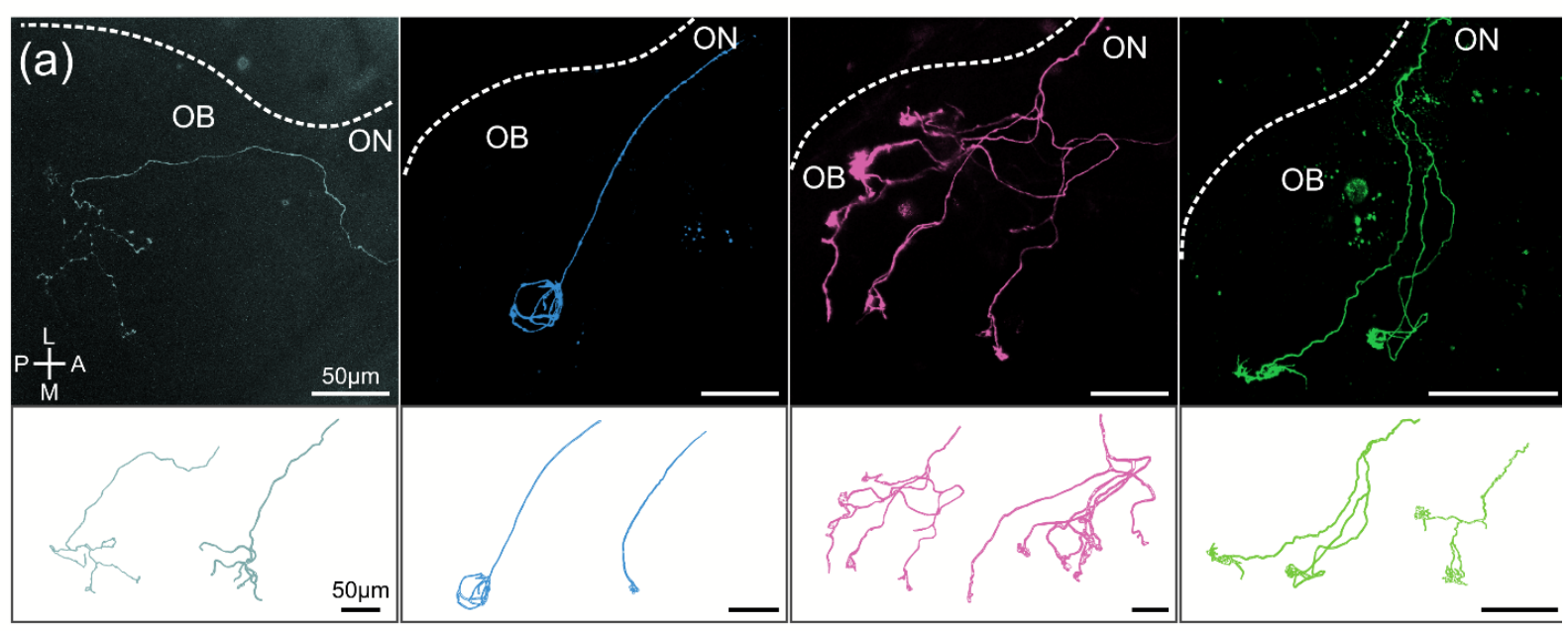

(b)
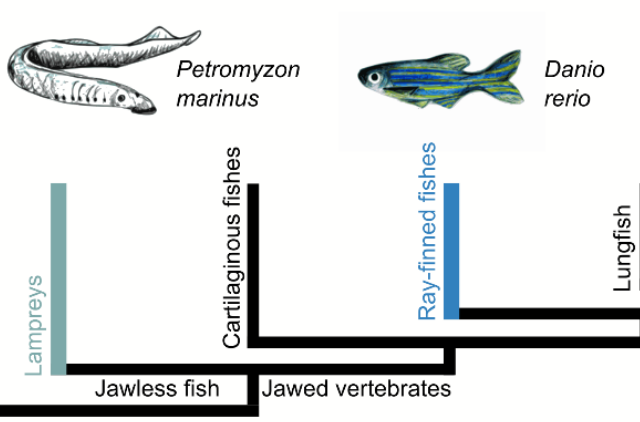

Ambystoma mexicanum

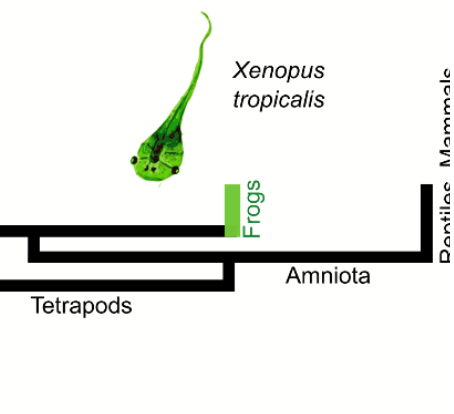

Figure 1 ORN axons in the OB of different aquatic vertebrates. (a) Single ORN axons of the juvenile sea lamprey (P. marinus; grey), zebrafish (D. rerio; blue), axolotl (A. mexicanum; magenta) and larval clawed frog ( $X$. tropicalis; green) show different levels of branching complexity. Sea lamprey and zebrafish axons are unbranched until they reach their terminals and only have a limited number of subbranches. Axolotl and frog axons branch in proximity to the $\mathrm{ON}$ and exhibit more subbranches. Axon tracings are shown from the transition between $\mathrm{ON}$ and $\mathrm{OB}$ until their terminals (upper panel). Dotted white line indicates the outline of the OB. The lower panel shows representative 3D reconstructions of two axons for each species. The first reconstruction of each species depicts the ORN axon shown in the upper panel. (b) The four examined species cover a broad evolutionary period from the divergence of the jawed vertebrates from their jawless ancestors to the emergence of the first tetrapods. All four species lead a fully aquatic lifestyle. OB olfactory bulb, ON, olfactory nerve, ORN olfactory receptor neuron, P posterior, A anterior, L lateral, M medial.

In addition to the amount, also the spatial distribution of branching points seems to be differently organized in fishes and amphibians (Figure 2c). The point of origin for distance measurements was set as the transition between $\mathrm{ON}$ and $\mathrm{OB}$. While most fish $\mathrm{ORN}$ axonal projections only arborize close to their terminals in the glomerular layer, amphibian axons start to bifurcate much closer to the ON and in the nerve layer (Figure 2c). The first lamprey axon bifurcation happens around $359 \pm 139 \mu \mathrm{m}$ after entering the $\mathrm{OB}$, which measures $61 \pm 12 \%$ of the distance from the origin to the furthest terminal point. The average unbranched axon segment of the zebrafish tracings was $184 \pm 39 \mu \mathrm{m}(81 \pm 12 \%)$, axolotl axons first branch at $85 \pm 33 \mu \mathrm{m}(18 \pm 5 \%)$ and the larval clawed frog axons at $94 \pm 40 \mu \mathrm{m}$ (45 $\pm 18 \%$ ). There is a significant difference between the relative position of the first bifurcation in axolotl 
axons and axons of both fish species (lamprey: $p=0.014$, zebrafish: $p=0.000001$ ). The position of the first bifurcations in Xenopus axons are not statistically different from the ones in lamprey or axolotl axons, but different from zebrafish ( $p=0.014$, Figure $2 c)$. There are substantial differences in the general ORN axon architecture between the four examined species. Our results clearly indicate that ORN axon bifurcations before reaching the glomerular layer cannot be attributed to an aquatic habitat, since this feature is absent both in the sea lamprey and the zebrafish. In contrast, our tracings suggest that this alternative branching pattern of bifurcations rostral to the glomerular layer could be linked more specifically to the amphibian lineage, since it was found to be present in both a salamander and a frog species.

(a)

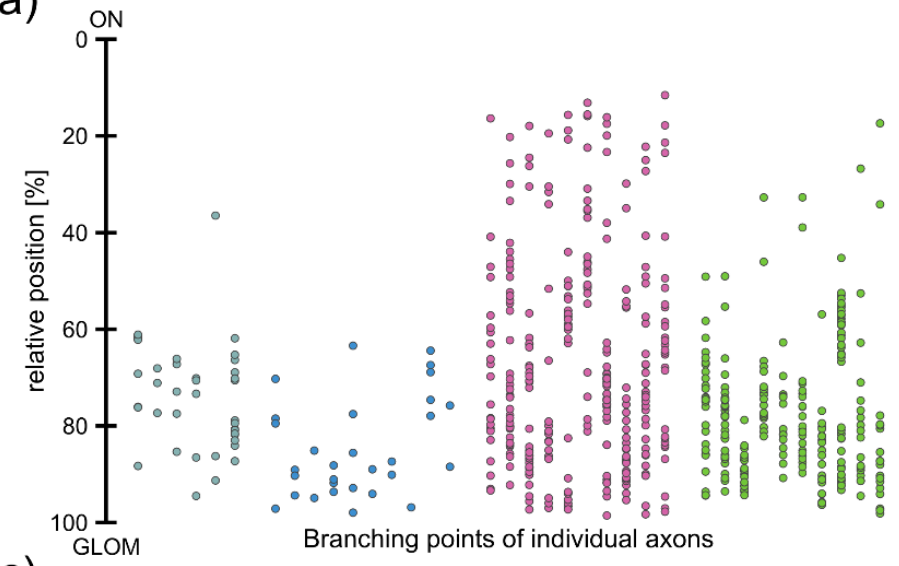

(c)

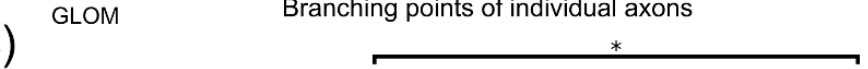

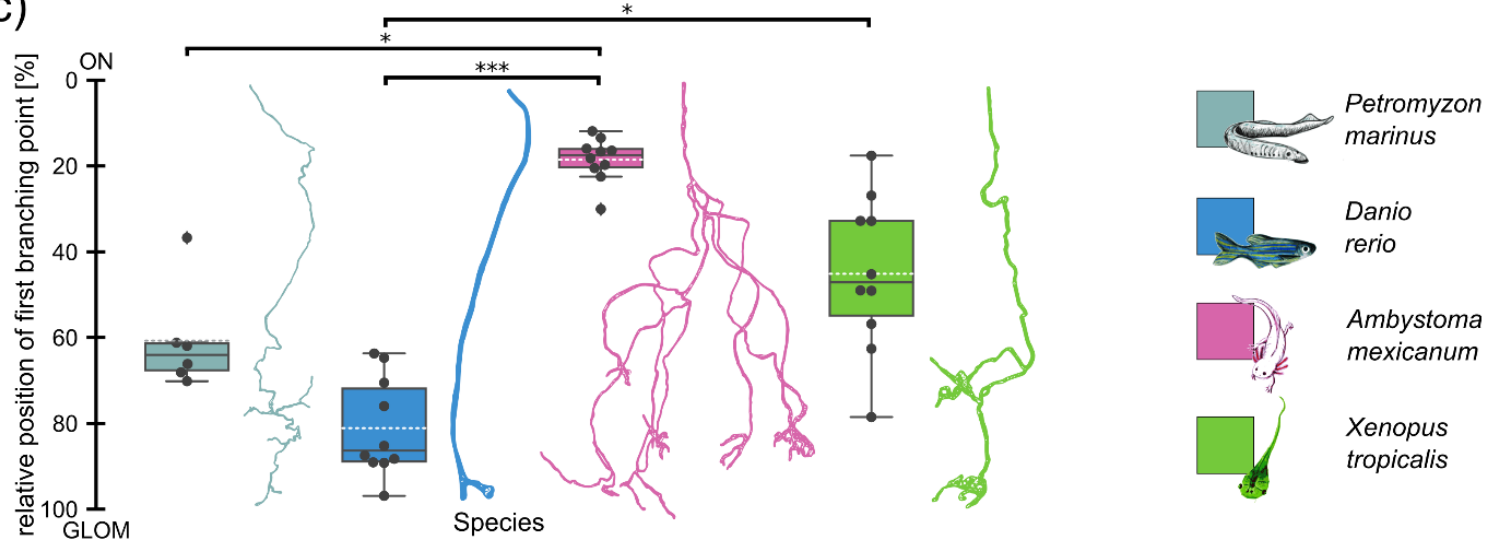

(b)

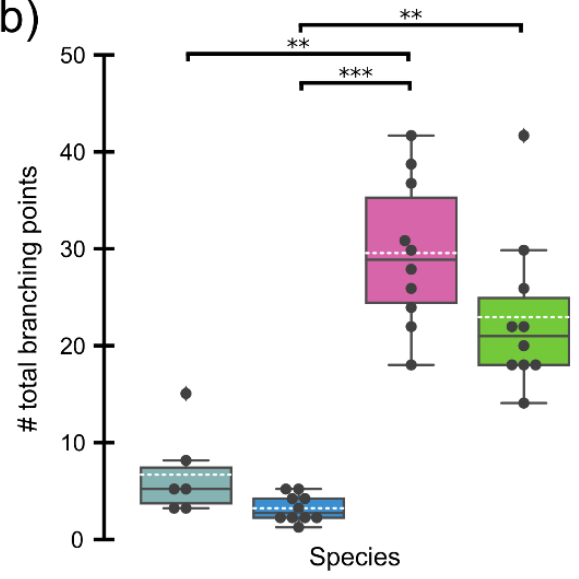

Figure $\mathbf{2}$ Number of axonal branching points and relative position of the first axonal bifurcation differ in fishes and amphibians. (a) Branching points of individual ORN axons and their relative position between the transition from $O N$ to $O B(0 \%)$ and their most distal axon terminal in the glomerular layer of the $O B(100 \%)$ are shown. All dots on a vertical line depict the positions of all branching points of a single reconstructed axon. ORN axons of sea lamprey ( $n=6$, five animals; grey), zebrafish ( $n=10$, ten animals; blue), axolotl $(n=10$, seven animals, magenta) and of the clawed frog ( $n=10$, eight animals; green) are shown. (b) Quantitative comparison of the total amount of ORN axonal branching points in each species. Each dot represents a single ORN axon. The black line indicates the median, the white dotted line the mean amount of branching points for axons of each species. Axolotl ORN axons have significantly more branching points than lamprey $(p=0.0058)$ and zebrafish axons $(p=$ 
0.00001). The ORN axons of the western clawed frog are significantly more branched than the zebrafish axons ( $p$ $=0.0013)$. (c) Species comparison of the first axonal bifurcation of ORN axons. The relative position of the first bifurcation between the transition from $O N$ to $O B(0 \%)$ and their most distal axon terminal (100\%) are shown. The relative position of the first branching point is closer to the axon terminals in the glomerular layer in the OB of both fish species. The axolotl axons branch in immediate proximity of the ON, significantly different from the fish axons (lamprey, $p=0.014$ and zebrafish $p=0.000001$ ). Frog axons also branch closer to the ON, significantly different from the zebrafish $(p=0.014)$. A representative axonal reconstruction is shown for each species. Statistical significance was tested using Kruskal-Wallis rank sum test followed by Dunn's multiple comparison post-hoc test with Holm-Bonferroni correction. OB olfactory bulb, ON olfactory nerve, ORN olfactory receptor neuron, GLOM glomerular layer.

\section{Multi-glomerular ORN axons are present in amphibians}

While it was reported for the rodent main olfactory system that single ORN axons project into a single glomerulus (Ressler et al., 1994; Vassar et al., 1994), 86\% of ORNs in the main OB of larval X. laevis were connected to more than one glomerulus (Nezlin and Schild, 2005). So far, comparative data of other vertebrate species on this matter are missing. To classify uni- and multi-glomerular ORN axons in the various animal species, we used an algorithm identifying glomerular clusters based on the spatial density of branching and endpoints of the reconstructed axonal structures.

We found that out of all fish axons (lamprey: $n=6$, zebrafish: $n=10$ ), only one zebrafish axon was classified as multi-glomerular, while the remaining axons projected into a single target structure and are thus considered uni-glomerular (Figure 3a and b). Only three fish axons (one lamprey axon and two zebrafish axons) had a single extra-glomerular branching point (average extra-glomerular branching points lamprey: $0.2 \pm 0.4$, zebrafish: $0.2 \pm 0.4$ ), while all other axons only started to arborize within the target glomerular cluster (Figure $3 \mathrm{c}$ ). The average distance from the transition between ON and OB to the glomeruli measured $401 \pm 111 \mu \mathrm{m}$ in sea lamprey and $194 \pm 41 \mu \mathrm{m}$ in zebrafish axons. In the axolotl, all except for one axon $(n=10)$ followed a multi-glomerular output pattern (Figure $3 a$ and $b)$, with four axons even innervating six distinct glomerular structures. The average axonal distance from the entry point in the $\mathrm{OB}$ to the glomeruli was $336 \pm 109 \mu \mathrm{m}$. Axolotl axons bifurcate $5.1 \pm 1.9$ times on average before reaching their target glomeruli (Figure $3 \mathrm{c}$ ), which is significantly different from both lamprey and zebrafish ( $p=0.0002$ and 0.00001 respectively).

The anuran olfactory system (represented by the clawed frog), showed a more heterogeneous wiring pattern. Three out of ten tracings were classified as uni-glomerular, while seven axons were multiglomerular. In comparison with the axolotl, Xenopus axons projected into maximally three glomeruli, while the majority $(n=6)$ displayed a bi-glomerular wiring pattern (Figure $3 a$ and $b)$. The mean axonal distance from the nerve to the glomeruli was $162 \pm 43 \mu \mathrm{m}$, the structures branched $1.6 \pm 1.0$ times before ending in glomerular clusters (Figure 3c). The branching density inside a single glomerular cluster was not significantly different between lamprey (6.3 \pm 4.7$)$, zebrafish (2.6 \pm 1.1 ) and axolotl (8.5 \pm 7.5). Xenopus glomeruli were more densely packed with sub-branches $(12.8 \pm 4.8)$ compared to zebrafish ( $p=0.00009)$, which displayed the lowest bifurcation density, often with only one branching point within a glomerulus. Our results support the hypothesis that multi-glomerular innervation of 
single ORN axons is not a wiring feature exclusive to $X$. laevis but seems to be common among other aquatic amphibian species of both salamanders and frogs. On the other hand, uni-glomerular wiring is the prevalent - almost exclusive - pattern in fishes, which strongly resembles the rodent wiring logic.

(a)

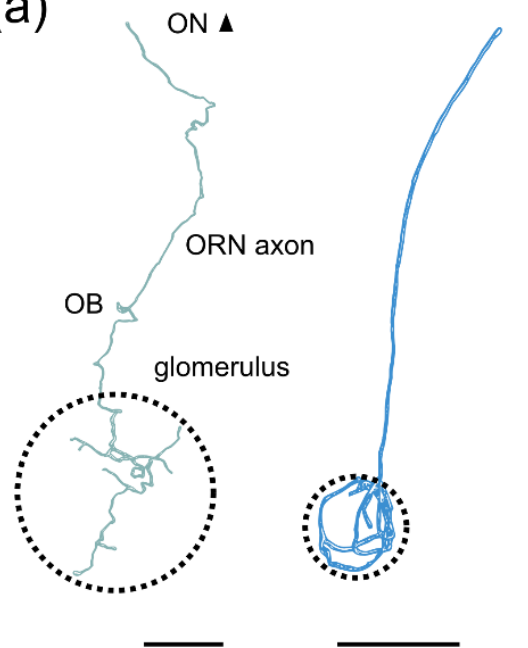

uni-glomerular

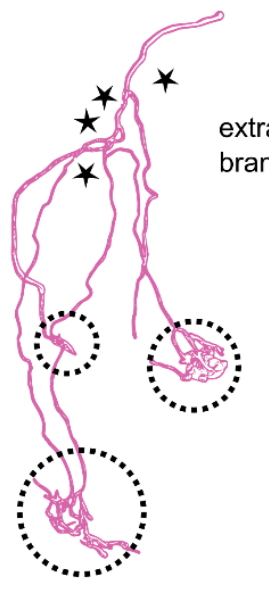

uni-glomerular

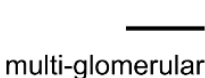

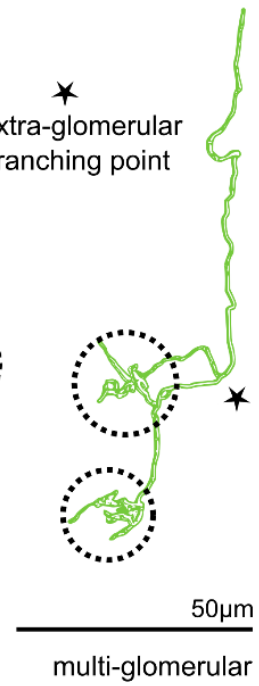

(c)

(b)
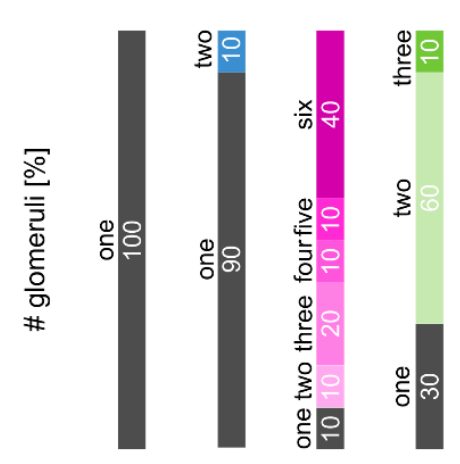

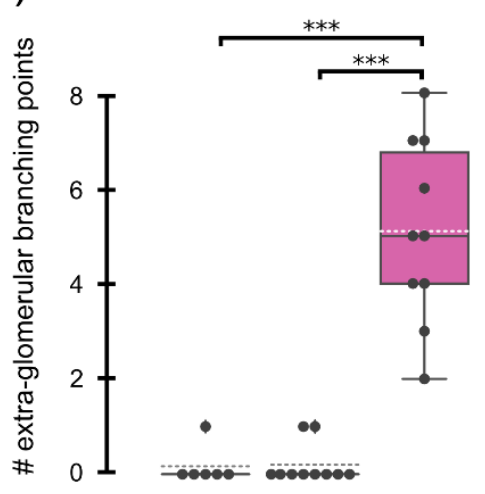

Ambystoma mexicanum
Figure 3 Lamprey and zebrafish ORN axons connect to a single glomerulus, amphibian ORN axons are multi-glomerular. (a) The representative ORN axonal reconstructions of the lamprey (grey) and the zebrafish (blue) both connect to a single glomerulus (dotted circle) and have no bifurcations prior to entering the glomerulus. The representative axolotl ORN axon (magenta) branches four times (black stars) before connecting to three glomeruli, the frog axon (green) has a single extra-glomerular branching point and innervates two separate glomeruli. (b) Population analysis of all examined axons, each stacked bar represents the percentual share of uniand multi-glomerular axons (two to six glomeruli) of one of the four species. All lamprey ORN axons ( $n=6$, five animals) and $90 \%$ ( $n=10$, ten animals) of zebrafish axons were classified as uni-glomerular by

the DBSCAN algorithm. On the contrary, most amphibian axons were classified as multi-glomerular (axolotl 90\%, clawed frog 70\%), with a varying number of innervated glomeruli. (c) Quantitative comparison of the amount of extra-glomerular branching points of all axons of each species. Each dot represents a single ORN axon. The black line indicates the median, the white dotted line the mean amount of extra-glomerular branching points. Axolotl axons have a significantly higher amount of extra-glomerular branching points than lamprey $(p=0.0002)$ and zebrafish ( $p=0.00001$ ). Statistical significance was tested using Kruskal-Wallis rank sum test followed by Dunn's multiple comparison post-hoc test with Holm-Bonferroni correction. OB olfactory bulb, ON, olfactory nerve, ORN olfactory receptor neuron. 


\section{The alternative wiring pattern in anurans is independent of developmental stage and ecology}

The alternative wiring pattern of multi-glomerular ORN axons has so far only been described in amphibians that live a water-bound lifestyle (Hassenklöver and Manzini, 2013). While all amphibian larvae are dependent on an aquatic habitat, most adult frogs leave the water after metamorphosis (Wells, 2007). We conducted ORN tracing experiments in anurans with more or less waterindependent adult lifestyles to test whether the alternative olfactory wiring is an olfactory adaptation of aquatic frogs and tadpoles or habitat independent. To account for the diverse ecology of the anurans, we examined the following species in addition to the aquatic $X$. tropicalis. Larval Rhinella arenarum are vegetarian grazers and adults are terrestrial. Both Scinax granulatus and Ranitomeya imitator have carnivorous/omnivorous tadpoles, their adults are arboreal and terrestrial, respectively. The major difference between these two species is that Ranitomeya provides extensive parental care and the number of tadpoles per parent pair is much lower than in Scinax, Rhinella and Xenopus (Figure 4) (Wells, 2007).
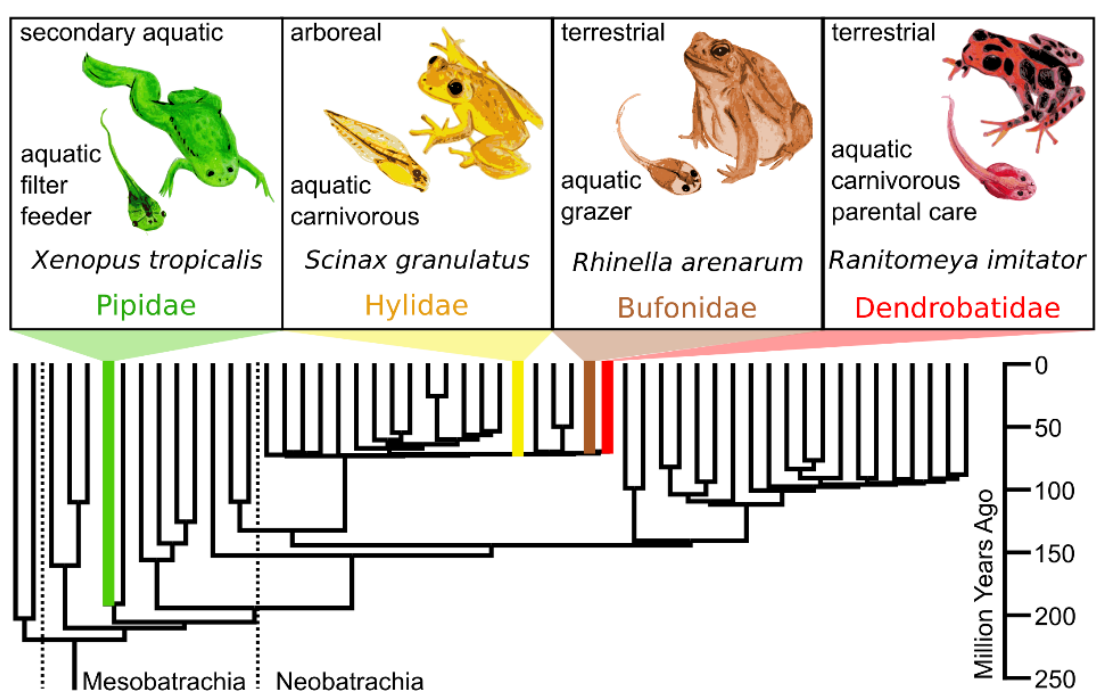

Figure 4 Overview about ecology and phylogeny of the four anuran species examined in this study. The clawed frog Xenopus tropicalis belongs to the Pipidae family and is classified as a Mesobatrachian, an evolutionarily more basal frog species. The other three species belong to the evolutionarily more 'modern' frogs, the Neobatrachians, and to the families of Bufonidae, Hylidae and Dendrobatidae. The diverse ecology of both the adult frogs (above) and their larval offspring (below) are summarized next to the drawings. The tree is pruned from the anuran tree in (Pyron, 2014), which originally included 3309 species.

The tracings of ORN axons in $R$. arenarum, $S$. granulatus and $R$. imitator tadpoles showed similar wiring and branching properties to those found in $X$. tropicalis tadpoles. In Figure 5 a two representative axonal reconstructions per species are shown. White stars indicate extra-glomerular bifurcations and white dotted circles highlight glomerular clusters. The number of branching points prior to entering the glomeruli was similar in all four species ( $X$. tropicalis: $1.6 \pm 1.0, \mathrm{n}=10$, eight animals; $R$. arenarum: $2.4 \pm 1.8, \mathrm{n}=8$, eight animals; $S$. granulatus: $1.4 \pm 1.2, \mathrm{n}=8$, six animals; $R$. imitator: $1.5 \pm 1.6, \mathrm{n}=6$, 
five animals), as was the distance of the first branching point relative to the entire length of the axon from the transition $\mathrm{ON}-\mathrm{OB}$ to the glomeruli (X. tropicalis: $45 \pm 18 \%, R$. arenarum: $43 \pm 26 \%, S$. granulatus: $41 \pm 28 \%$, $R$. imitator: $45 \pm 19$ ).

To exclude that the alternative multi-glomerular wiring pattern is linked to the larval stages and/or their aquatic lifestyle, we conducted sparse cell electroporation in juveniles of the terrestrial $R$. arenarum and the arboreal $S$. granulatus. Figure $5 b$ shows reconstructions of single ORN axons of juveniles of the two species. Their morphology does not significantly differ from the morphology of conspecific tadpoles nor from the other larval axons examined. Before reaching the glomeruli, axons of juvenile $R$. arenarum and S. granulatus bifurcate $2.2 \pm 1.5$ ( $n=5$, two animals) and $1.6 \pm 1.1$ ( $n=7$, five animals), respectively. The first bifurcations occur at $48 \pm 29 \%$ (R. arenarum) and $28 \pm 16 \%$ (S. granulatus) of the total distance between the $\mathrm{ON}-\mathrm{OB}$ transition and the axon terminals. The only parameter that slightly differs from axons of their larval conspecifics (other than an approx. 1.5-fold increase in total axonal length from tadpoles to juveniles) is the amount of branching points inside the glomerular clusters ( $R$. arenarum: larval $5.7 \pm 1.4$, juvenile $3.8 \pm 0.9$, S. granulatus: larval $11.7 \pm 3.1$, juvenile $5.5 \pm 4$ ).

All four anuran species and ontogenetic stages were heterogeneous with regard to the number of glomerular clusters that are innervated by a single axon and the proportion between uni- and multiglomerular axons. We categorized the axonal structures based on recurring branching patterns and classified them into five categories (type $A$ to $\mathrm{E}$; Figure $5 \mathrm{c}$ and d). Among the uni-glomerular axons, we distinguished two main types. Type $A$ is characterized by a single, unbranched axon terminating in a single glomerular cluster. This is the prevailing type reported in rodents and also found in the fish species we examined here. Type B also terminates in a single glomerulus but has at least two separate branches projecting into the same glomerular structure. Of all the axons reconstructed from larval and juvenile anurans ( $n=44$ ), only $11 \%$ belonged to Type A. Type B was more frequent, amounting to $32 \%$ of the axons. $57 \%$ of the axons were classified as multi-glomerular, with $50 \%$ of all axons innervating two glomeruli and only three axons (7\%) innervating more than two glomerular end-structures (Type E). In $23 \%$ of all axons two glomerular clusters were innervated by a single branch each (Type C), in 27 $\%$ of the tracings, at least one of the two glomeruli was innervated by more than one sub-branch (Type $D$, Figure $5 c$ ). Among the axons traced in the various species, all species displayed at least four out of the five different types.

We additionally measured the differences in the length of branches entering the same or different glomeruli of a single axonal structures for all the anuran species. We found 27 cases ( 20 in larvae, 7 in juveniles) where a single glomerulus was innervated by at least two separate axonal branches (Figure $5 \mathrm{~d}$, left plot). By subtracting the shortest innervating branch from the longest, we measured a branch length difference of $46 \pm 71 \mu \mathrm{m}$ in tadpoles and $56 \pm 27 \mu \mathrm{m}$ in juveniles. On average, the shortest branch had $76 \pm 16 \%$ the length of the longer one. This value was consistent between tadpoles ( $75 \pm$ $16 \%)$ and juveniles ( $80 \pm 12 \%)$. In all multi-glomerular axons ( $n=25$, larvae 19 , juveniles 6$)$, we measured the branch length difference between the branches innervating the nearest and the furthest glomerulus (Figure 5d, right plot). In larval anurans, the difference was $58 \pm 46 \mu \mathrm{m}$, in juveniles $154 \pm$ 
$214 \mu \mathrm{m}$. The distance to the glomerulus closer to the nerve layer was $53 \pm 30 \%$ of the distance to the glomerulus most distant from the nerve layer (tadpoles: $48 \pm 31 \%$; juveniles: $67 \pm 25 \%$ ).

The collected data indicates that ORN axonal projections in larval and juvenile amphibians are much more heterogeneous than what has been reported in rodents and what we found in fishes. In amphibians, multi-glomerular innervation is retained throughout their developmental stages and does not seem to be linked to a specific lifestyle or habitat.

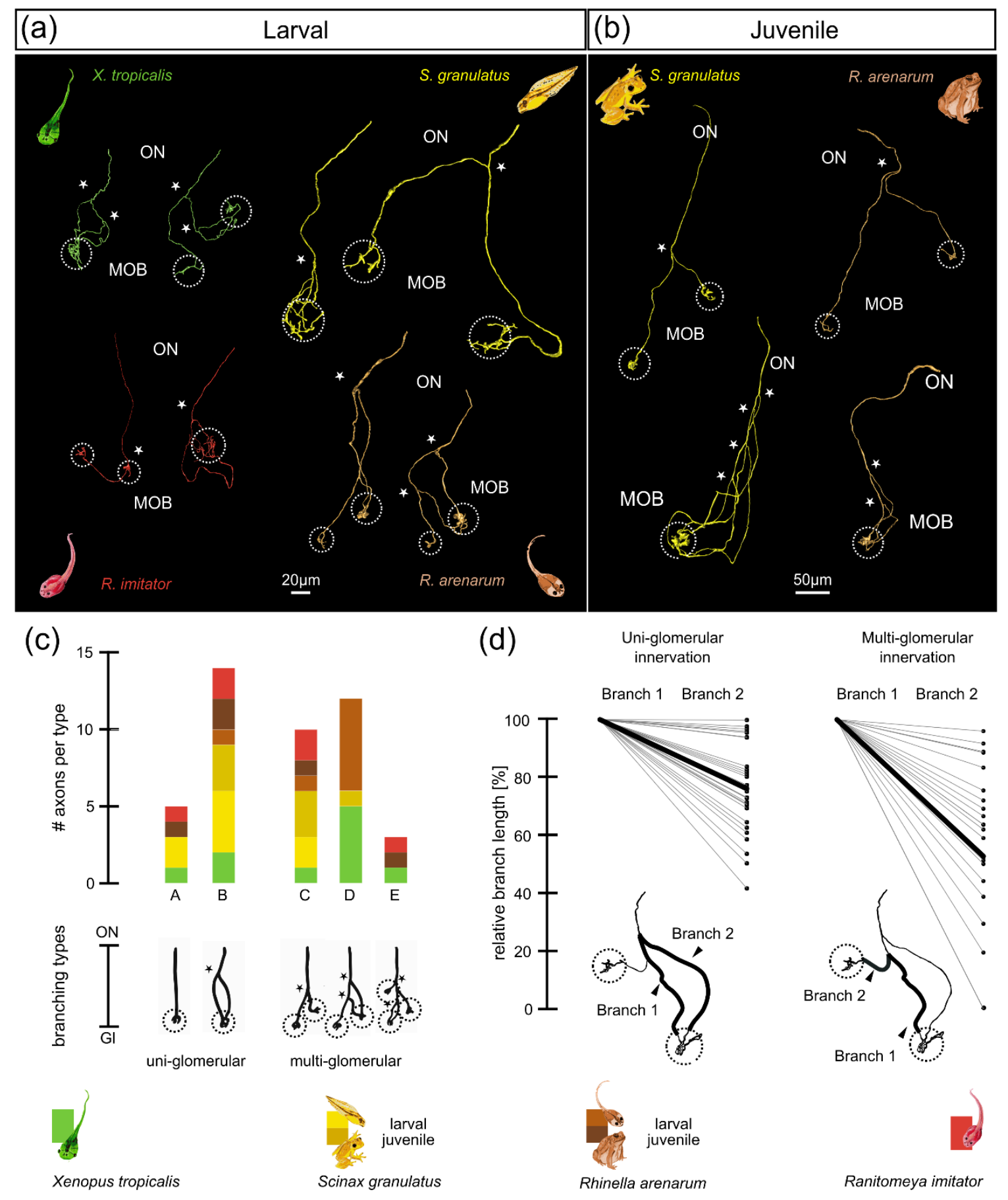


Figure 5 Comparison of the ORN axon morphologies in different larval and juvenile frog species with different lifestyles. (a) Two representative ORN axon reconstructions are shown for tadpoles of each of the anuran species $X$. tropicalis, $R$. arenarum, $R$. imitator and S. granulatus. Each axon was reconstructed starting from the transition between $\mathrm{ON}$ and main $\mathrm{OB}$ to the most distal axon terminal. Multiple axonal branches innervate one or two glomeruli (white dotted circles), and generally have one or two extra-glomerular branching sites (white stars). (b) Two representative ORN axon reconstructions are shown for post-metamorphotic terrestrial juveniles of $R$. arenarum and arboreal S. granulatus. Axonal structures are comparable to their larval counterparts shown in A, one or two glomeruli are innervated by two or more axonal subbranches. (c) ORN axons were classified into five branching types ( $\mathrm{A}$ and $\mathrm{B}$ uni-glomerular, C - E multi-glomerular). Types are schematically shown below, black stars indicate extra-glomerular branching points. The number of axons belonging to each branching type is shown in the stacked bar plot above. We included all ORN axons of tadpoles ( $X$. tropicalis, green, $n=10$, eight animals; S. granulatus, light yellow, $\mathrm{n}=8$, six animals; $R$. arenarum, light brown, $\mathrm{n}=8$, eight animals; $R$. imitator, red, $n=6$, five animals) and juveniles (S. granulatus, dark yellow, $n=7$, five animals; $R$. arenarum, dark brown, $n=5$, two animals). The counts of axons per type for each species and developmental stage are marked according to the color legend below. (d) Relative length of the longer and the shorter branch of individual ORN axons innervating the same glomerulus (left plot) or two different glomeruli (right plot). Each pair of dots refers to a single axon, where branch 1 is the longer branch and branch 2 is the shorter one. The thick black line visualizes the mean length relation between the two branches. In uni-glomerular axons, the shorter branch measures $76 \%$ of the length of the longer branch ( $n=27$, ORN axons of all species and developmental stages included). In multiglomerular axons, the shorter branch measures on average $53 \%$ the length of the longer branch innervating a different glomerulus ( $n=25$, ORN axons of all species and developmental stages included). MOB main olfactory bulb, ON, olfactory nerve, ORN olfactory receptor neuron

\section{Emergence of the bifurcation of ORN axon coincides with the first tetrapods}

In an attempt to put our findings into an evolutionary context, we compared the wiring properties of the fishes and urodela to the anuran juveniles, to give an overview about the presumably mature system of the animals. Since the axolotl is a neotenic salamander, the main olfactory system of the one to two-month-old larva is assumed to be a mostly developed system that does not undergo drastic changes until sexual maturity is reached. The majority of the fish axons (75\%) are uni-glomerular and unbranched prior to entering the glomeruli (Figure 6, blue bars), which is in accordance with the prevailing vertebrate wiring principle. The juvenile anurans are the most heterogeneous group. Only the minority of axons follow the unbranched axon principle (8\%). Most axons branch at least once before terminating in one or more glomeruli. $42 \%$ of the axons innervate one glomerulus with more than two separate branches, 50\% innervate more than two glomeruli (Figure 6, yellow bars). Of the salamander axons, $80 \%$ innervate more than three glomerular structures (Figure 6, magenta bars). None of the axolotl axons followed the unbranched one axon-one glomerulus principle.

Our results strongly imply an alternative principle of odor processing on the level of the OB and that the emergence of this principle coincides with the divergence of the first terrestrial tetrapods from their aquatic ancestors. Even though data from the smallest order of modern amphibians (Caecilians) is still elusive, axonal bifurcations and multi-glomerular innervation seem to be conserved in all amphibians regardless of their developmental stage and habitat. 
(a)

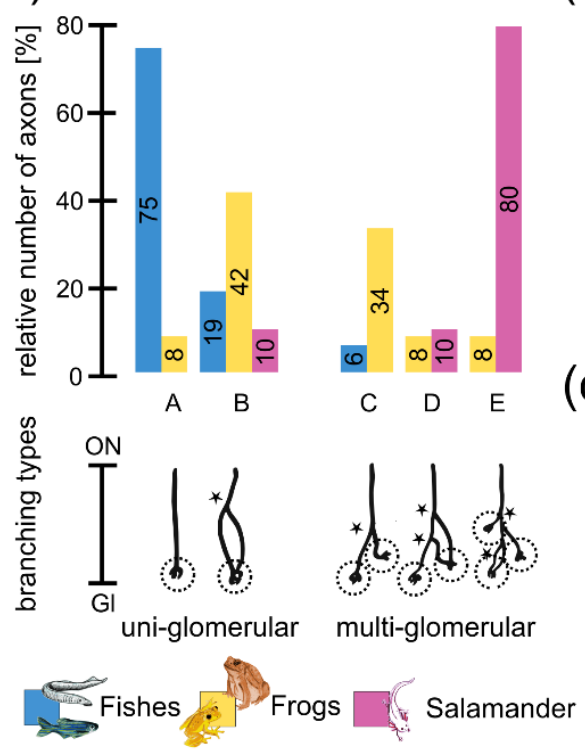

(b)

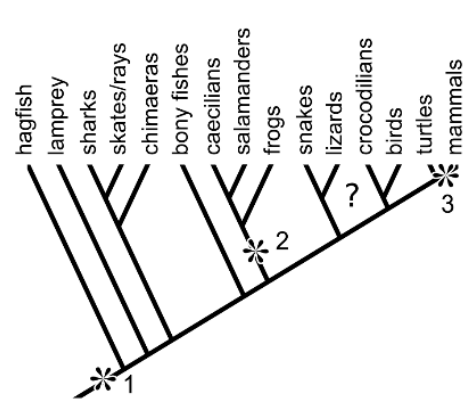

(c)

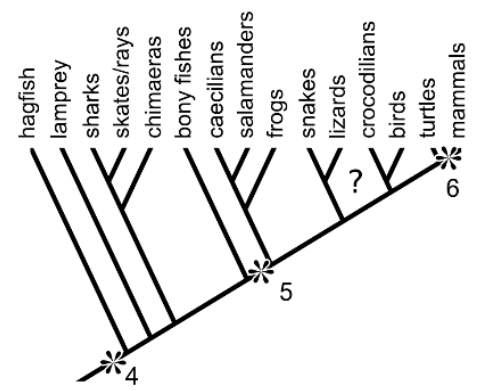

Figure 6 Multi-glomerular innervation pattern is a conserved feature of amphibians and could have emerged with the evolution of the first tetrapods. (a) ORN axons of fish species (lamprey and zebrafish, blue bars, $\mathrm{n}=$ 16), juvenile frogs ( $R$. arenarum and $S$. granulatus, yellow bars, $n=12$ ) and the axolotl salamander (magenta bars, $n=10$ ) were classified into five branching types, (A and $B$ uni-glomerular, C-E multi-glomerular). Types are schematically shown, black

stars indicate extra-glomerular branching points, dotted circles indicate glomeruli. The relative number of axons belonging to each branching type for each group is shown in the bar plots. The majority of fish axons follow the unbranched axon pattern (type A) and the majority of salamander ORN axons innervate three or more glomeruli (type E). Frog axons are more heterogeneous with the most represented types being bi-glomerular (type C) and uni-glomerular with at least one extra-glomerular branching point (type B). (b) One possible evolutionary scenario for the emergence of the multi-glomerular ORN projections could be that the unbranched, uniglomerular ORN axon is a basic vertebrate trait $\left({ }^{*} 1\right)$ and has secondarily evolved into a multi-glomerular alternative wiring logic in the amphibian lineage $\left({ }^{*} 2\right)$ and independently also in the accessory system of mammals (*3). (c) In a second scenario, the basic unbranched ORN wiring logic ( $\left.{ }^{*} 4\right)$ changed to the alternative bifurcating axon logic in vertebrates at the transition from an aquatic to a terrestrial habitat $(* 5)$. In mammals, this alternative logic then segregated to the accessory system, while the main olfactory system again followed the unbranched axon wiring logic $(* 6)$. The phylogenetic tree was modified from Eisthen, 1992.

\section{Discussion}

\section{Multi-glomerular innervation is absent in fishes and present in all developmental stages of amphibians}

The consensual hypothesis regarding olfactory wiring in the vertebrate main olfactory system is established on the idea that all ORNs expressing one allele of the olfactory receptor gene repertoire transmit information into only one or two glomeruli in the main OB via an unbranched axon. This leads to the formation of a precise odotopic map, where each glomerulus is part of a unique olfactory unit that encodes the information detected by a specific receptor type (Mombaerts, 2006; Ressler et al., 1994; Vassar et al., 1994). In this study we show that ORN axons of post-larval sea lamprey and zebrafish clearly follow the unbranched axon principle postulated for all vertebrates (Mombaerts, 2006; Ressler et al., 1994; Vassar et al., 1994). Fish axons solely arborize within their target-glomerulus 
and only one axon out of 16 has been found to innervate two distinct glomeruli in the zebrafish. In contrast, $57 \%$ of ORN axons labeled in anurans show connections to multiple glomeruli $(90 \%$ in the salamander species examined).

It is still under debate how the precise wiring between ORN axons and projection neuron dendrites within the glomeruli is established and whether there are extensive pruning mechanisms taking place during development. Experiments in newborn rabbits (Yilmazer-Hanke et al., 2000) and mice (Marcucci et al., 2011; Tenne-Brown and Key, 1999) have shown that exuberant ORN axon growth in the main OB during early development is subsequently pruned. Contrastingly, other studies conducted in neonatal rats (Klenoff and Greer, 1998) and zebrafish embryos (Dynes and Ngai, 1998) show that ORN axons arborize to their final morphology without erroneous targeting. Tenne-Brown and colleagues demonstrate the occurrence (15\% of all axons) of extra-glomerular bifurcations and multi-glomerular connections of single ORN axons in neonatal mice, but only until two weeks after birth (Tenne-Brown and Key, 1999). Of the above mentioned studies, only Klenoff and colleagues report the occurrence of axons arborizing in two multiple glomeruli in rats after the initial pruning phase is over, yet only as a very rare exceptions ( $<0.1 \%$ of axons) (Klenoff and Greer, 1998).

In contrast, we found that multi-glomerular wiring is preserved even after metamorphosis is finished in all examined amphibians. $50 \%$ of the axons analyzed in juveniles of $R$. arenarum and $S$. granulatus innervated more than one glomerulus and $92 \%$ of the axons bifurcated before entering the glomerular structures. A study conducted in the main $\mathrm{OB}$ and the $\mathrm{AOB}$ of larval and post-metamorphotic Xenopus laevis (Hassenklöver and Manzini, 2013) has yielded very similar results, showing that neither preglomerular bifurcations nor multi-glomerular innervation can be solely attributed to the larval stages. However, in accordance with the results shown by Marcucci and colleagues (Marcucci et al., 2011), we noticed a reduction of the total number of branching points from larval to post-metamorphotic animals. Even though the number of extra-glomerular bifurcations (larval $1.7 \pm 1.4, n=32$; juvenile 1.8 $\pm 1.3, n=12$ ) remained constant, the arborizations inside a single glomerulus decreased in the juveniles (larval $10.1 \pm 5.1$; juvenile $4.8 \pm 3.1$ ). While the reduction of branches inside a glomerulus can be explained by pruning mechanisms during development, multi-glomerular innervation in the amphibian main $O B$ is not strictly linked to an immature larval stage.

\section{The alternative wiring logic in amphibians has parallels to the rodent $A O B$}

In the rodent accessory olfactory system, the axons of VRNs expressing the same vomeronasal receptor type converge onto 15-30 glomeruli, constituting a more vague spatial code than in the main OB (Belluscio et al., 1999; Dulac and Axel, 1995; Rodriguez et al., 1999). In contrast to the main system, the postsynaptic partners of the ORN axons, the projection neurons, extend several dendrites into multiple glomeruli. It is still unclear whether they integrate between input of VRNs expressing different or the same vomeronasal receptors (Belluscio et al., 1999; Del Punta et al., 2002b; Rodriguez et al., 1999; Wagner et al., 2006). Several attempts have been made to unravel the AOB wiring logic from VRN axons to projection neuron dendrites in rodents. A study investigating genetically labeled receptor neurons expressing a single type of V1R or V2R in mice gives evidence for a homotypic connectivity model. In this model, a single projection neuron extends its dendrites into multiple glomeruli 
innervated exclusively by the same vomeronasal receptor type (Del Punta et al., 2002b). Another study supported a selective heterotypic connectivity model in which a single projection neuron receives information from glomeruli that get sensory input not from a single vomeronasal receptor type, but from closely related receptors within a receptor subfamily (Wagner et al., 2006).

The discrepancies between the wiring principles in the rodent main $\mathrm{OB}$ and $\mathrm{AOB}$ suggest that there might be different aspects of odor information extracted by the respective subsystems. It has additionally been reported that approx. $10 \%$ of single VRN axons in the nerve layer of the AOB split into several sub-branches, reaching out to multiple glomeruli (Larriva-Sahd, 2008). The wiring pattern of amphibians described in our study therefore resembles the rodent accessory system rather than the main system. While the rodent system shows a clear separation into the OR-type receptor expressing MOE and the V1R and V2R expressing VNO, this segregation is incomplete in amphibians. Among the OR-type receptors and other receptor gene families, the MOE of Xenopus was shown to express V1Rs as well as early diverging V2Rs, while the Xenopus VNO is expressing more recently diverging V2R genes (Bear et al., 2016; Date-Ito et al., 2008; Syed et al., 2013).

In line with this heterogeneous receptor gene expression in the amphibian MOE, we found quite a heterogeneity in ORN axon branching patterns. Different branching types could be connected to different receptors. The mix of ORs and VRs in the MOE of amphibians could explain the heterogeneity of branching patterns, while in the more segregated rodent system, axonal bifurcations and a more vague spatial glomerular code are only found in the accessory system. It has already been shown that olfactory receptors are involved in axon guidance and the formation of the glomerular map (Feinstein and Mombaerts, 2004; Mombaerts et al., 1996). Still, the expression of vomeronasal receptors in the MOE of amphibians is unlikely to be the only cause for axonal bifurcations and the alternative wiring pattern. V1Rs and V2Rs are already expressed in the sensory epithelium of fishes, but we could show that ORN axon bifurcations are absent in both lampreys and zebrafish. It is more plausible that the mechanisms by which the receptors influence axonal guidance could have changed over evolutionary time and that the new wiring principle has only emerged after the divergence of the first tetrapods from the aquatic ancestors.

In addition to the classification of ORNs based on odorant receptor expression, the majority of ORNs belong to one of two morphological types: ciliated and microvillous ORNs. While the rodent main OB is primarily innervated by ciliated ORNs, the $A O B$ receives axonal input from the microvillous ORNs residing in the VNO (Munger et al., 2009). In the zebrafish olfactory system, ciliated ORNs project to the dorso-medial region of the $\mathrm{OB}$, while microvillous cells innervate the lateral glomerular clusters in a mutually exclusive way (Sato, 2005). In addition to the two major cell types, the olfactory epithelium of teleosts also contains crypt cells (Hansen and Finger, 2000; Olivares and Schmachtenberg, 2019). Kappe neurons (Ahuja et al., 2015) and pear-shaped olfactory neurons (Wakisaka et al., 2017) have been found in zebrafish, their presence in other teleosts is not confirmed yet (Olivares and Schmachtenberg, 2019). Glomerular projections to the OB of zebrafish are also very heterogeneous, forming smaller and larger clusters which are invariant across individuals (Baier and Korsching, 1994; Braubach et al., 2012). Given the diversity in ORN types and projections, it is conceivable that some 
ORNs deviate from the unbranched uni-glomerular pattern described in the present work. Further studies will be necessary to address this issue.

The MOE of the Axolotl contains both types of ORNs, similar to adult salamanders (Eisthen et al., 1994; Reiss and Eisthen, 2008). Larval anurans characteristically have a MOE equipped with both ciliated and microvillous ORNs and a VNO exclusively containing microvillous ORNs (Hansen et al., 1998; Taniguchi et al., 1996). The MOE of adult anurans solely consists of ciliated ORNs while the receptor neurons in the VNO are microvillous (Bloom, 1954; Reese, 1965). Since both uni- and multi-glomerular ORN axons were found in the juvenile frogs in the present study, it seems quite unlikely that either of the patterns is linked exclusively to ciliated or microvillous ORNs.

\section{Multi-glomerular ORN innervation is mirrored by projection neuron morphology in vertebrates and invertebrates}

Just like the projection neurons in the rodent $\mathrm{AOB}$, projection neurons in the main $\mathrm{OB}$ of amphibians and reptiles extend multiple dendrites into multiple glomeruli, where they terminate in dendritic tufts (Dryer and Graziadei, 1994). In the case of amphibians, the multi-glomerular projection neuron morphology seems to be mirroring the multi-glomerular ORN axons described in this study. In the sea lamprey, the morphology of the single ORN axons we found show a net like branching structure within single glomeruli. This morphology is also mirrored in the uni-glomerular arborizations of the lamprey projection neurons (Green et al., 2013). In many teleost fish species, it is known that projection neurons extend many primary dendrites into multiple glomeruli. However, this was only shown to be the minority in zebrafish (Fuller et al., 2006). In accordance with these results, we also show that single zebrafish ORN axons mostly (90\%) terminate in a single glomerulus. It is intriguing to speculate, whether teleost fishes equipped with multi-glomerular projection neurons also display multiglomerular ORN wiring.

A similar mirror image in the connectivity pattern between ORN axon and postsynaptic projection neurons occurs in the evolution of the antennal lobe in orthopteran insects (Hansson and Stensmyr, 2011). It was shown that in more basal orthopterans (e.g. the great green bush cricket), single receptor neuron axons are innervating a single glomerulus and a single projection neuron extends its dendrite into one sole glomerulus, resembling the mammalian main system. Contrastingly, in later diverging orthopterans (locusts and grasshoppers) both single receptor neurons as well as projection neurons connect to multiple glomeruli - a pattern similar to amphibians (Ernst et al., 1977; Hansson and Stensmyr, 2011; Ignell et al., 2001). The multi-glomerular pattern in locusts is linked to the formation of a high number of micro-glomeruli ( 2500 glomeruli), while the one-to-one pattern in basal orthopterans is linked to fewer number of bigger glomeruli ( 40) (Ignell et al., 2001). A similar evolution towards micro-glomeruli could have taken place among vertebrates: the sea lamprey has very large but few glomeruli (41-65) (Frontini et al., 2003), the zebrafish has approx. 140 quite differently sized glomeruli (Braubach et al., 2012) and Xenopus laevis has a larger number of smaller glomeruli, 350 in the main OB (Manzini et al., 2007) and 340 in the AOB (Nezlin and Schild, 2000). The concept of the 
branched and multi-glomerular ORN axon has thus developed at least twice independently, however its putative functional implications remain elusive.

Interestingly, the human $\mathrm{OB}$ also has a larger number of glomeruli ( $>5000)$ when compared to the rodent system ( 2000 ), even though humans only have $\sim 350$ intact olfactory receptor genes compared to 1100 in rodents (Maresh et al., 2008). It is therefore conceivable that glomerular wiring might also be quite different among different mammalian species.

\section{Different evolutionary scenarios for the emergence of the alternative wiring logic}

Branched receptor neuron axons with multi-glomerular innervation have been shown in about $10 \%$ of VRNs in the mouse AOB (Larriva-Sahd, 2008) and as a predominant type in the main OB (Nezlin and Schild, 2005) and the AOB of Xenopus laevis tadpoles, as well as adults (Hassenklöver and Manzini, 2013). In this study we show that this wiring logic is also present in the axolotl salamander and in anuran tadpoles of four ecologically distinct families (Pipidae, Bufonidae, Hylidae and Dendrobatidae) as well as terrestrial post-metamorphotic frogs. From our results, we can conclude that the multiglomerular ORN wiring pattern is independent of tadpole or adult ecology and seems to be a feature derived from the common ancestor between frogs and salamanders, since it is also present in axolotl. In both the jawless and bony fish species we examined, we could not find any clear signs of the presence of this alternative wiring principle. However, it needs to be mentioned that zebrafish is a late diverging teleost species and - given the diversity of extant bony fishes - it might not be representative for all bony fish. We can thus not exclude that different strategies of glomerular wiring have also developed in this group.

From an evolutionary perspective, our results suggest several plausible scenarios, two of which will be discussed here. First, the axonal bifurcations of ORNs could have developed independently in amphibians ( $* 2$, Figure $6 \mathrm{~b})$, and in the VNO of mammals ( $* 3$, Figure $6 \mathrm{~b})$, with the ancestral vertebrate trait being the one-to-one wiring logic $(* 1$, Figure $6 \mathrm{~b})$. The presence of multi-glomerular wiring structures in the orthoptera supports the idea that this alternative wiring could have a functional advantage in odor processing and has thus evolved independently multiple times (Hansson and Stensmyr, 2011). In the second scenario, the multi-glomerular pattern could have evolved around the divergence of the terrestrial tetrapod lineage from its aquatic ancestors ( $* 5$, Figure $6 \mathrm{c}$ ), coinciding with the first occurrence of the VNO (Eisthen, 1992) and a huge expansion and reshaping of the OR-type gene family (Bear et al., 2016; Niimura and Nei, 2006). Given that the odorant receptor is directly influencing axon targeting and the formation of glomeruli (Feinstein and Mombaerts, 2004; Mombaerts, 2006), it could potentially also have an impact on axonal branching. Newly emerging receptors or axon branching principles after the tetrapod divergence could be responsible for this alternative wiring and be more widespread in the MOE and VNO of earlier diverging tetrapods (i.e. amphibians), and more focally expressed in the VNO of mammals ( ${ }^{*} 6$, Figure $\left.6 c\right)$. 


\section{Conclusions}

Taken together, our results show that the prevailing idea of an unbranched ORN axon arborizing only in a single glomerulus cannot be generalized for vertebrates any longer. While jawless and bony fishes display the one-to-one wiring logic that is shown for the rodent main olfactory system, our results indicate that bifurcating ORN axons and multi-glomerular wiring are a general feature of the amphibian olfactory system. This alternative wiring scheme is neither linked to larval stages, nor to a specific habitat or lifestyle of amphibians. It cannot be excluded that this feature is even more common amongst vertebrates and that it constitutes the basis of an alternative way for odor processing.

\section{Acknowledgements}

We thank all the present and past members of the Manzini laboratory for fruitful discussion and input, especially Thomas Offner and Sara Joy Hawkins. We thank Anja Schnecko for dedicated animal care and Gianfranco Grande and Eva Fischer for support to set up experiments. L.W. was granted a travel fellowship (JEBTF-180809) by the company of Biologists Limited and the Journal of Experimental Biology to visit the O'Connell lab. This work was supported by DFG Grant 4113/4-1, the bilateral cooperation program CONICET-DFG-MINCYT 23120160100031CO, UBACYT 20020170200191BA and in part, by Award Number S1ORR02557401 from the National Center for Research Resources (NCRR). Its contents are solely the responsibility of the authors and do not necessarily represent the official views of the NCRR or the National Institutes of Health.

\section{Author Contributions}

Conceptualization, L.W., T.H. and I.M.; Investigation, Formal Analysis, Visualization and Writing Original Draft, L.W.; Writing - Review \& Editing, L.W., L.D.J., A.G.P., B.S.Z., L.A.O., T.H. and I.M.; Funding Acquisition and Resources, L.D.J., A.G.P., B.S.Z., L.A.O., T.H. and I.M.; Supervision, T.H. and I.M.

\section{Ethics approval and consent to participate}

All experiments performed followed the guidelines of Laboratory animal research of the Institutional Care and Use Committee of the University of Windsor (AUPP 14-05), University of Buenos Aires (CD: 316/12, Protocol \#22), the University of Göttingen (33.9-42502-04-12/0779), University of Gießen, (GI 15/7, 932_GP) and Stanford University (APLAC-33016).

\section{Consent for publication}

No human subjects; not applicable.

\section{Competing interests}

The authors declare that they have no competing interests 


\section{Chapter 6: General Discussion}

Olfaction has demonstrated the remarkable evolutionary capability to adapt to environments that are as contrary as the ocean floor and the tree summits in the rainforest. Research focusing on the sense of smell in a single species occupying a single ecological niche broadens our understanding of general olfactory processes but withholds some parts of the story. To contribute to our understanding of evolutionary variability in the olfactory system, the present work focuses on a group of animals that are inherently linked to two separate environments both aquatic as well as terrestrial environment: the amphibians (Duellman and Trueb, 1994; Reiss and Eisthen, 2008; Thewissen and Nummela, 2008).

In the first part of this Chapter, I will discuss the relative functional importance of the various olfactory subsystems identified and described in Chapters 3 and 4, both in development and comparatively between species. In the second part, I will describe the evolutionary differences in the projection patterns of single RNs from the MOE to the $\mathrm{OB}$ and the possible functional implications (Chapter 5 ).

\section{The relative importance of olfactory subsystems in anurans}

It is widely unknown how the immense variation in brain architecture among species evolved and to what extend it is shaped by environmental selection pressure (Jerison, 1973). The relative size of the OBs have been used as an estimate of olfactory capabilities in many vertebrates. (Corfield et al., 2015; Finlay and Darlington, 1995; Gonzalez-Voyer et al., 2009; Yopak et al., 2010, 2015). In some birds, extensive foraging and migration behavior has been shown to correlate with a bigger $\mathrm{OB}$, while the development of higher cognitive functions in parrots and corvids is associated with less use of the olfactory sense (Corfield et al., 2015; Emery, 2006). Similarly, oceanic migratory sharks as well as deepsea sharks had the relatively largest OBs among cartilaginous fishes, suggesting that they rely quite heavily on their sense of smell (Yopak et al., 2015). In anurans, little attention has thus far been paid to the relative importance of the sense of smell in general or its respective subsystems. Anuran brains are believed to have a quite uniform brain structure (Jerison, 1973) with only minor environmentdriven adaptations described so far (Taylor et al., 1995). Nevertheless, anurans pose a formidable group of animals to explore environment-driven effects on the structure of sensory systems because of their unique bi-phasic lifestyle. Intriguingly, it has also been shown that selection pressure has had separate effects on the tadpole and the adult animal, which led to specific adaptations and adaptive radiation in both ontogenetic stages independently (Bossuyt and Milinkovitch, 2000).

\section{Variability vs. stability in the larval olfactory system}

Although direct-development has evolved multiple times among anurans (Heinicke et al., 2009), most anuran species have a free swimming aquatic tadpole stage (Wells, 2007). The aquatic habitat is however not uniform and tadpoles have radiated into several microhabitats (Altig and McDiarmid, 1999; Roelants et al., 2011). In an attempt to describe morphology as a function of habitat, Altig and Johnston have sub-categorized several ecomorphological guilds of tadpoles (Altig and Johnston, 1989). 
They majorly describe feeding related types e.g. the benthic type, which relies on rasping off vegetation from the ground, carnivorous tadpoles or filter feeders, but also categorized classes of tadpoles living in still water (lentic) vs. streams and rivers (lotic) (Altig and Johnston, 1989). Since one major use of olfaction in tadpoles is related to finding food (Altig and McDiarmid, 1999), it seems plausible that the olfactory systems of above described ecomorphotypes show some adaptive features. In the absence of comparative physiological data showing which chemosensory subsystem is detecting specific odorant cues in tadpoles, information is limited to mostly behavioral and anatomical data (Jungblut et al., 2017; Quinzio and Reiss, 2018).

The blueprint of a peripheral larval olfactory organ mostly consists of an external nasal opening leading into the principal nasal chamber containing the MOE and connecting to the buccal cavity via the choana (Reiss and Eisthen, 2008; Figure 6A). The VNO is situated in a side pocket of the principal cavity (Reiss and Eisthen, 2008). Nevertheless, differences in the structure and size of the olfactory organs exist among species (Benzekri and Reiss, 2012; Hansen et al., 1998; Jermakowicz et al., 2004; Jungblut et al., 2011, 2012; Nowack and Vences, 2016). In Ascaphus truei, which belongs to the earliest diverging anurans (Archaeobatrachians), tadpoles have a patch of sensory epithelium lining a small lateral appendix of the principal cavity, a structure that is only rarely described and so far completely enigmatic (Benzekri and Reiss, 2012). In addition, 'epithelial bands' were described to be embedded into the roof of the buccal cavity (Benzekri and Reiss, 2012). These buccal exposed patches of epithelium have also been described as part of the ventral MOE in some bufonid species (Jermakowicz et al., 2004; Jungblut et al., 2017; Figure 6A ), hylids (Jungblut et al., 2017) and ceratophryids (Quinzio and Reiss, 2018).The buccal exposed epithelium was hypothesized to sample oral cues in the buccal cavity and could be a complement to the taste system (Jungblut et al., 2017; Wassersug, 1980). In larval Rhinella arenarum, a bufonid from South America, this patch of ventral MOE even occupies $20-25 \%$ of the entire epithelial volume, but it is completely absent in the filter feeding tadpoles of Xenopus (Jungblut et al., 2017). While it is known that the RNs residing in the VNO of tadpoles generally project axons towards glomeruli in the AOB (Jungblut et al., 2012; Figure 6A), the respective projection targets of the ventral/buccal exposed epithelium, putatively within the $M O B$, are completely unexplored.

Considering the variability of the olfactory organ and ecomorphotypes of different anuran larvae (also presented in Chapter 3, Figure 1), I was interested to understand how this translates to the central level and the organization of olfactory projections in the MOB (Chapter 3). So far, a clear account of the glomeruli in larval amphibians is limited to larvae from the genus Xenopus (Gaudin and Gascuel, 2005; Manzini and Schild, 2010; Manzini et al., 2007; Nezlin et al., 2003). Xenopus laevis is generally quite distinct from many anurans. The phylogenetic divergence between the Mesobatrachians and the 'more modern' Neobatrachians happened in the Triassic period around 200 million years ago (Roelants et al., 2011). Additionally, Xenopus tadpoles lack keratinized mouthparts, restricting their diet to smallest planktonic food particles in the water (Altig and Johnston, 1989; Altig and McDiarmid, 1999). Accepting their olfactory system and glomerular projections as an 'anuran blueprint' thus seems simplistic and needs comparative verification. However, we found the glomerular organization of anuran larvae to be remarkably conserved (Figure 6A). 
The tracing experiments presented in Chapter 3 show that glomeruli of all anuran larvae examined are segregated into four clusters. The nomenclature and cluster identification was based on the data from (Manzini et al., 2007) and (Gaudin and Gascuel, 2005). In comparison to these studies, it is to note that we grouped some of the smaller clusters defined in Gaudin and Gascuel together and restricted our identification to four bigger clusters. Our data closely replicates the data from these studies conducted in Xenopus laevis. We identified that about $40 \%$ of the glomerular volume in the MOB is grouped together as a lateral cluster (LC) and about the same volume forms a dorso-medial cluster (DC). The remaining $20 \%$ fall on the middle and intermediate cluster (MC and IC). Interestingly, the LC is significantly bigger than the DC in both Xenopus laevis and Xenopus tropicalis tadpoles, while the opposite scenario is the case in the four neobatrachian species. With the knowledge on the presence of segregated functional processing streams and their putative receptors and intracellular signaling pathways known from Xenopus, I will speculate on the implications of these differences.

In Xenopus laevis, there are two well described parallel processing streams in the MOB. Medially located glomeruli are relying on $\mathrm{G}_{\text {oolf }}$ and the cAMP-dependent intracellular signaling pathway and are mostly tuned to aldehydes, ketones and alcohols as ligands. This pathway is putatively linked to ciliated RNs and OR-type olfactory receptors (Gliem et al., 2013). The laterally located glomeruli on the other hand are putatively relying on $\mathrm{G}_{\alpha 0 / \mathrm{i}}$ linked to vomeronasal receptors or TAARs (Gliem et al., 2013). The lateral processing stream is CAMP-independent and shows responses to amino acids (Manzini and Schild, 2003; Manzini et al., 2002). The same processing streams could also be observed in tadpoles of Xenopus tropicalis (own unpublished observations), which suggests an evolutionary conservation of the glomerular functionality at least among the pipid tadpoles. A difference in the relative volume of the LC or the DC could thus be linked to a shift in importance of the two pathways in the respective species. Whether the more pronounced DC in the four examined neobatrachian species reflects an adaptation to e.g. their feeding strategies remains to be proven.

The formation of a glomerular map through axonal projections to the olfactory bulb relies heavily on the expression of olfactory receptors expressed in the dendrites, but also the axons of RNs (Barnea et al., 2004; Mombaerts, 2006). It is thus possible that the differences in the volume of the clusters reflects a different composition of the olfactory receptor repertoire of the species. Xenopus laevis has an unusually high number of V2R genes (Hagino-Yamagishi et al., 2004; Shi and Zhang, 2007) also in comparison to other amphibians (Kiemnec-Tyburczy et al., 2012). This might play a big role in the development of the lateral processing stream. V2Rs in Xenopus are not restricted to the VNO as it is in mammals (Syed et al., 2013), and it is known from fish V2Rs that they are particularly sensitive to amino acids (Speca et al., 1999). No other anuran receptor genome has been evaluated in detail. It is thus conceivable that differences in gene numbers of a receptor family could be linked to a different number of RNs expressing these receptors and thus different numbers of axonal projections. It is to note however, that the distribution of $\mathrm{G}_{\alpha \mathrm{olf}}$ and $\mathrm{G}_{\alpha \mathrm{o}}$ in the $\mathrm{MOB}$ of larval Rhinella arenarum does not show a clear bipartition into medial and lateral stream (Jungblut et al., 2009). It is thus even more interesting to see whether the anatomical clusters are also functionally similar or just form a similar morphological frame for a completely distinct odorant map (Figure 6A). 


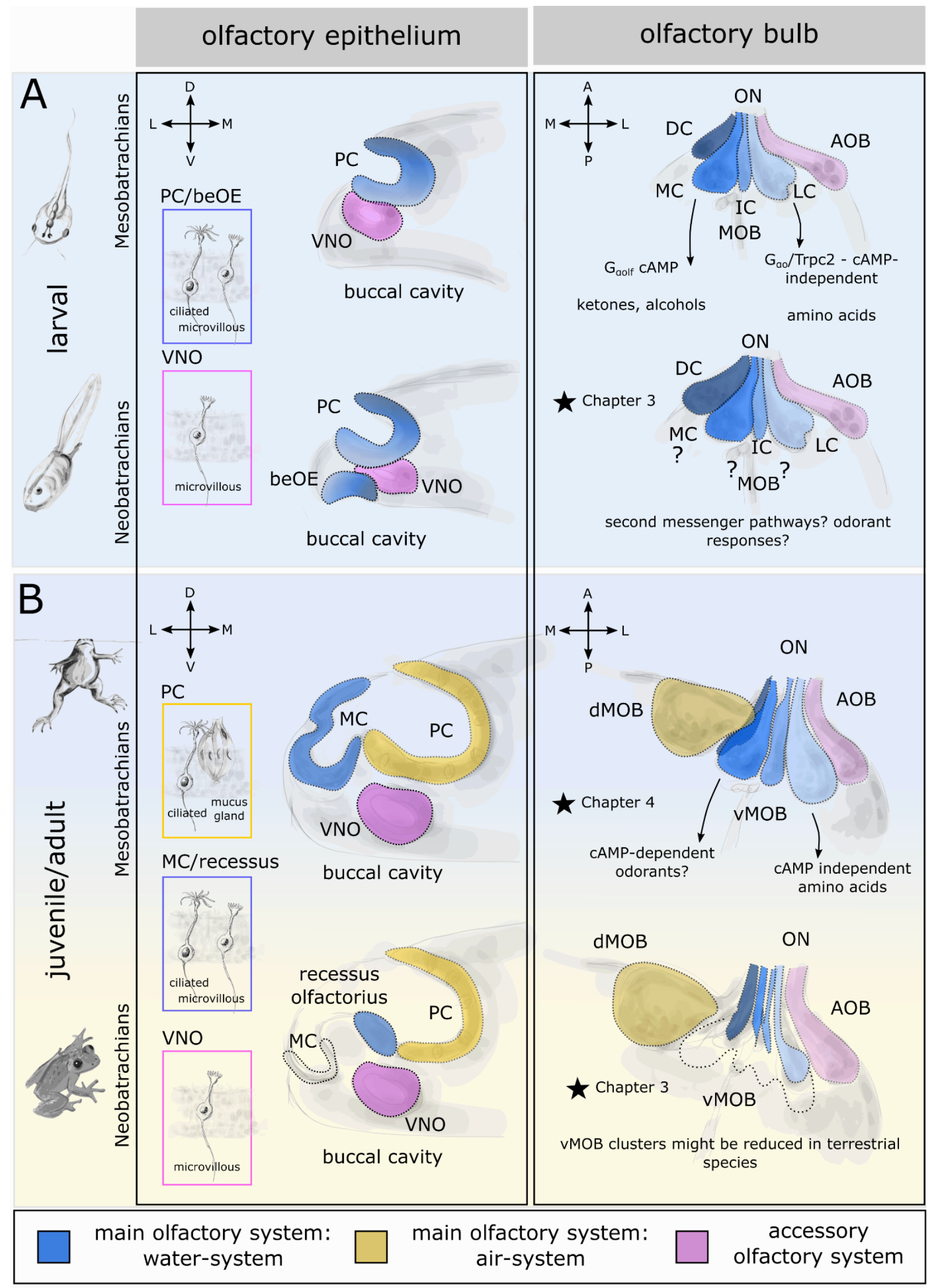


Figure 6 Pathways for aquatic and aerial olfaction in anurans.

A) Differences in the larval olfactory system between Xenopus laevis tadpoles (above) representing early diverging Mesobatrachians and Rhinella arenarum tadpoles (Neobatrachians; below). The olfactory organs are distinct by the presence of a buccal exposed epithelium (beOE) in R. arenarum. Both show a segregated VNO$A O B$ pathway (magenta). The boxes indicate the putative morphotypes of RNs in the respective epithelia. Glomerular clusters are indicated by the dotted lines. Even though the olfactory periphery and the lifestyle of the tadpoles varies, the glomerular clusters are remarkably conserved (*Chapter 3 ). B) Simplified olfactory pathways in adults of the aquatic Xenopus laevis and terrestrial Neobatrachians are depicted. While Xenopus has a 'water-type' epithelium lining the MC, the putative Neobatrachian equivalent is the recessus olfactorius. The $\mathrm{MC}$ is generally non-sensory in Neobatrachians. From the larval to the adult condition, a dorsal portion of $\mathrm{MOB}$ (dMOB) forms during metamorphosis (*Chapter 4$)$. Terrestrial frog species might have a less developed ventral 'water bulb' than the aquatic Xenopus, possibly as a terrestrial adaptation (*Chapter 3 ). A anterior, AOB accessory olfactory bulb, beOE buccal exposed epithelium, D dorsal, DC dorsal cluster, dMOB dorsal main olfactory bulb, IC intermediate cluster, L lateral, LC lateral cluster, M medial, MC(periphery) middle cavity, MC(OB) medial cluster, $\mathrm{MOB}$ main olfactory bulb, MOE main olfactory epithelium, OB olfactory bulb, ON olfactory nerve, $\mathrm{P}$ posterior, $\mathrm{PC}$ principal cavity, $\mathrm{V}$ ventral, $\mathrm{VMOB}$, ventral main olfactory bulb, VNO vomeronasal organ. The stars indicate results obtained in the course of this thesis.

Furthermore, the projection targets of the RNs in the buccal exposed epithelium present in many species is not known (Figure 6A). The receptors involved in this putatively distinct subsystem are also still elusive. Of the species I described in Chapter 3, the presence of this epithelium is verified only in $R$. arenarum (Jungblut et al., 2017). The presence of this epithelial patch in Scinax granulatus could be assumed based on its phylogenetic and ecological closeness to Hypsiboas pulchellus, which has an even larger buccal exposed epithelium than Rhinella arenarum (Jungblut et al., 2017). Of the olfactory organ structure of tadpoles of Ranitomeya variabilis and Dendrobates tinctorius, nothing is known to my knowledge. I could thus speculate that the projection of a possible buccal exposed epithelium in the four neobatrachian species accounts for a bigger glomerular volume of the DC. At this point, comparative data is too scarce to make clear correlations between habitat and olfactory morphology. Nevertheless, the data described in Chapter 3 suggests that the glomerular clusters underly strong developmental constraints and this glomerular configuration is presumably adapted to a variety of habitats. With the lack of functional data in other species than X. laevis (and Xenopus tropicalis, unpublished results), it is purely speculative to assume that the anatomical segregation might also reflect similar functional subsystems present in all examined tadpoles (Figure 6A).

\section{Variability vs. stability in the metamorphotic and postmetamorphotic olfactory system}

The selective pressure to adapt to a specific habitat has already shaped the anurans in their larval stage and their ecomorpho-space (Roelants et al., 2011). The metamorphosis from an aquatic to a mostly terrestrial organism however demands another level of adaptive strategies (Reiss and Eisthen, 2008). In this section I will discuss the metamorphotic changes happening from the aquatic tadpoles of Xenopus laevis to the secondarily aquatic adult form (Chapter 4) and from the tadpoles of Dendrobates tinctorius to the terrestrial adult (Chapter 3 ). I will particularly put this data into the context of aquatic vs. aerial olfaction (Figure 6B). Adult anurans exhibit a varying degree of terrestriality, from species 
that never enter the water or only to deposit their eggs to completely aquatic species (Wells, 2007). The structure of the olfactory organ is described in detail in many different anuran species.(Benzekri and Reiss, 2012; Föske, 1934; Helling, 1938; Jermakowicz et al., 2004; Jungblut et al., 2011, 2017; Nowack and Vences, 2016; Paterson and Hindle, 1951; Quinzio and Reiss, 2018).

\section{The water-system}

There are several parts of the olfactory epithelia positively correlated with either aquatic or terrestrial lifestyle. Most neobatrachian species exhibit a separated part on the anterior floor of the MOE, called the recessus olfactorius, which lacks the glands associated with the aerial system and resembles a larval type 'aquatic' epithelium (Nowack et al., 2013; Figure 6B). Its involvement in aquatic olfaction has been suggested by Helling (1938), who found the recessus to be much larger in species that are more dependent on the water (Helling, 1938). The extend of this recessus varies between less than $1 \%$ of the total epithelial volume in the terrestrial $R$. arenarum (Jungblut et al., 2017) and about $30 \%$ in the semiaquatic Telmatobius hauthali (Helling, 1938). The correlation between a well-developed recessus and the aquatic habitat was demonstrated even more conclusively in a comparative study conducted in terrestrial and aquatic members of the Ceratophryidae (Quinzio and Reiss, 2018). There are several other epithelia which are hypothesized to be homologous to the recessus: the anterior part of the PC in the early diverging Archaeobatrachian Ascaphus truei (Benzekri and Reiss, 2012), the MC in Xenopus (Föske, 1934) and the accessory cavity of Pipa americana (Trahms, 1936). Since most Neobatrachians also have a MC lined with non-sensory epithelium, the homology of the organs is disputed. The field agrees however on their involvement in aquatic olfaction (Reiss and Eisthen, 2008).

Despite the enormous amount of anatomical accounts on amphibian noses, little attention has been paid to the neuronal circuits involved in the respective water and air system on the MOB level. In Chapter 4, I presented a thorough analysis of the changes of RN projections from the periphery to the MOB during metamorphosis in Xenopus laevis with a special focus on the connectivity patterns and the neuronal circuit involved. It is known from different studies using soybean agglutinin, that RNs in the adult MC project to the ventrally located glomeruli in the VMOB (Franceschini et al., 1992; Key and Giorgi, 1986; Meyer et al., 1996; Figure 6B). The glomeruli of the vMOB after metamorphosis closely resemble the glomerular organization in the premetamorphotic tadpole of $X$. laevis. In the larva, the VMOB glomerular clusters are formed by RN axons from the PC epithelium (Manzini and Schild, 2010; Nezlin et al., 2003). In a step by step analysis of the glomerular clusters of the MOB during metamorphosis Gaudin and Gascuel (2005) showed a remarkable constancy of the glomerular organization. I analyzed the development of the $\mathrm{VMOB}$ - the 'water-system' - more closely and investigated the shift from being innervated by the larval PC, to the adult MC by sparse cell labelling. Indeed, the shift seems to happen gradually between premetamorphosis (stag 51/52) and metamorphotic climax (stage 60/61). During this period, the overall glomerular organization does not change. Results presented in Chapter 4 suggest that the constancy of glomerular organization is due to a perfect balance between the apoptosis of larval PC-projections (Dittrich et al., 2016; Higgs and Burd, 2001) and incoming axonal projections from newly formed RNs in the MC. 
Since metamorphotic tadpoles still need to feed and are not exempt from predation risk, it is to assume that they must rely on a properly working olfactory system. Indeed, the functional odor map described in previous work by our group (Gliem et al., 2013; Manzini and Schild, 2010; Manzini et al., 2002) was still remarkably in place in the glomeruli of the 'water-bulb' during metamorphosis. The lateral, amino acid-sensitive and CAMP-independent processing stream and the medial CAMP-dependent stream were distinguishable in tadpoles up to stages 60/61. Furthermore, behavioral experiments in differently staged tadpoles show, that attraction to amino acids did not change up to metamorphotic climax. These experiments imply that at least the coarse functional organization of the VMOB and its putatively behavioral output might remain unaffected by the high amount of cell death happening in parallel (Dittrich et al., 2016).

Even though it remains to be elucidated, which exact behavioral functions the two streams fulfil, they seem to be developmentally hardwired at least in the Xenopus olfactory system. Intriguingly, an aminoacid sensitive lateral stream was found in the $O B$ of most fish species and was shown to be related to feeding behavior in the carp (Hamdani, 2001a, 2001b). A clear feeding-related behavioral effect of amino acids in Xenopus is still not clear (Terni et al., 2017). Also, functional experiments in this thesis were only conducted up until stage 61 . Between stage 61 and the end of metamorphosis, the feeding habit of Xenopus changes from an herbivorous filter feeder to an omnivorous/carnivorous predatorial juvenile. It seems plausible, that this trophic shift might be accompanied with a change in olfactory processing. Further experiments and functional imaging using food odorants instead of single components might be needed to understand to what extend the olfactory map changes during metamorphosis.

In contrast to most other anurans, Xenopus adults also exclusively live in the water. The maintenance of a 'larval-type' nose tuned to detect waterborne stimuli like amino acids or bile acids might thus be related to their lifestyle. The necessity to build or maintain an adult 'water-nose' is less obvious in terrestrial species. We took the terrestrial Dendrobates tinctorius as an example to understand whether the VMOB projections might be habitat dependent in adult anurans (Chapter 3 ). It is known from dendrobatids that airborne olfaction plays an important role in orientation and homing behavior (Forester and Wisnieski, 1991), but clear use of waterborne olfactory cues are not described. Our tracing experiment showed, that while the glomerular clusters in the VMOB in Xenopus tropicalis remain morphologically intact during metamorphosis, the $\mathrm{VMOB}$ of postmetamorphotic Dendrobates tinctorius shows signs of degeneration or vestigialization in comparison to the larval animals (Figure $6 \mathrm{~B})$. Even though the lateral glomerular cluster remains visible, especially the $\mathrm{MC}$ an IC have almost disappeared. The most plausible reason for this degeneration could be the absence of the recessus olfactorius, which was described as absent for Dendrobates tinctorius by Helling (1938). More comparative data will be needed to understand how the ventral 'water-bulb' and its function are correlated to the presence or absence of the respective water-epithelia (Figure 6B). Also, the connection of the recessus olfactorius to the glomeruli in the MOB is completely unknown so far. 


\section{The air-system}

Conversely to the smaller and more anatomically diversified 'water-systems', the major part of the principal nasal cavities of adult anurans is covered by an epithelium consisting of ciliated RNs and mucus producing glands - the 'air-nose' (Bloom, 1954; Reese, 1965; Reiss and Eisthen, 2008; Figure 6B, yellow). In terrestrial frogs, detection of airborne cues is mostly involved in foraging (Shinn and Dole, 1978, 1979) and homing behavior (Forester and Wisnieski, 1991; Grubb, 1975). Similar to the correlation between a bigger recessus olfactorius and the aquatic lifestyle, an elevated ridge (eminentia olfactoria) on the floor of the principal cavity was positively correlated with a higher degree of terrestriality (Helling, 1938; Quinzio and Reiss, 2018). Its exact function or distinct connectivity to the olfactory bulb remains enigmatic up to date. Most accounts of the olfactory bulb of adult anurans describe a clear connectivity between the MOE and the glomeruli in the MOB (Scalia et al., 1991a, 1991b). The MOB of anurans is usually described as a laminar neuronal structure fused at the interhemispheric midline (Eisthen and Polese, 2007; Herrick, 1910; Jiang and Holley, 1992b; Leveteau, 1992; Scalia et al., 1991a, 1991b; Figure 6B).

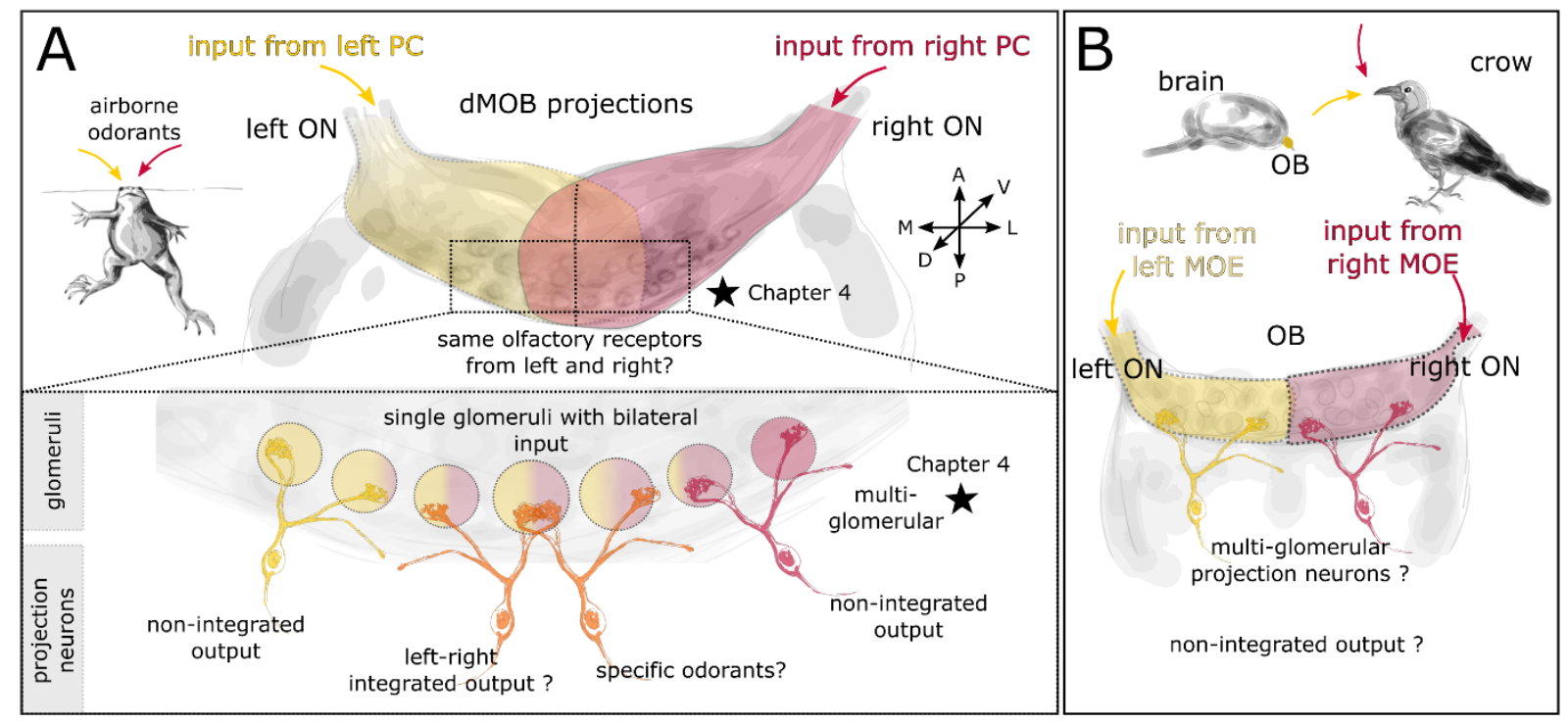

Figure 7 Left-right integration in the anuran air-bulb.

A) In postmetamorphotic anurans, the two MOBs are fused at the interhemispheric midline and form a single glomerular projection field. In anurans, there is substantial overlap in the innervation from the left and right MOE (upper panel; *Chapter 4). On a glomerular level, individual glomeruli around the midline are innervated bilaterally and projection neurons putatively integrate the input coming from the left and the right air-nose. B) OB midline fusion is also present in passeriform birds, but no overlap between the projections could be detected (Yokosuka et al., 2009a), which suggests a different processing logic and function in birds and frogs. A anterior, $\mathrm{D}$ dorsal, dMOB dorsal main olfactory bulb, L lateral, M medial, main olfactory epithelium, OB olfactory bulb, ON olfactory nerve, $\mathrm{P}$ posterior, $\mathrm{PC}$ principal cavity, $\mathrm{V}$ ventral. The stars indicate results obtained in the course of this thesis. 
In most studies, no distinction between the dorsal and ventral MOB was made. This could possibly be due to a relatively small size of the $\mathrm{VMOB} \mathrm{glomerular} \mathrm{clusters,} \mathrm{as} \mathrm{an} \mathrm{artefact} \mathrm{of} \mathrm{histological} \mathrm{slicing} \mathrm{or}$ due to the absence of the ventral 'water-bulb' in terrestrial species. Xenopus laevis is an aquatic frog, with putatively terrestrial ancestors (Reiss and Eisthen, 2008; Wells, 2007). The presence of a welldeveloped aerial olfactory system implies that the adult frog is well adapted also to smell on land (Föske, 1934). During metamorphotic remodeling, newly generated RNs residing in the PC epithelium no longer project to the glomeruli in the $\mathrm{VMOB}$, but start to project to a dorso-medial target region starting from approx. stage 50 (Gaudin and Gascuel, 2005; Reiss and Burd, 1997a; Figure 6B, yellow). Data presented in Chapter 4 suggest that the extend of the $\mathrm{dMOB}$ projections already occupy more space than the 'water-bulb' projections around stage 54, at the onset of prometamorphosis. After the end of metamorphosis, the axonal projections of the 'air-bulb' occupy approx. $70 \%$ of the entire glomerular volume of the MOB. The metamorphotic timeline of axonal projections in the terrestrial frog Dendrobates tinctorius is quite similar to the ones in Xenopus laevis and tropicalis. While during premetamorphotic stages RN axons solely project to the glomerular clusters in the $\mathrm{VMOB}$, the de novo formed dMOB grows extensively until the end of metamorphosis (Chapter 3, Figure 3).

Apart from the size differences, the dorsal 'air-bulb' differs significantly from the $\mathrm{VMOB}$ in its wiring properties (Figure 7A). It has been occasionally noted, that some RN axons in the dMOB of Xenopus innervate contralateral glomeruli (Ebbesson et al., 1986; Leveteau, 1992; Reiss and Burd, 1997a). I quantified the percentage of overlap between projections coming in from the left and right ON and found a quite linear increase starting around $0 \%$ during premetamorphosis and reaching approx. $40 \%$ by the end of metamorphosis (Figure 7A). Additionally, single glomerular structures often contained axon terminals from both sides. This suggests that postsynaptic projection neurons could integrate between input from the left and right nose on a single tufted dendrite (Figure 7A). Alternatively, a single projection neuron could get input from the left and the right side at the multiple dendritic tufts (Figure 7A). Comparative anatomical data from different anurans suggests that the fused $\mathrm{dMOB}$ is an ancestral trait and shared at least between the Meso- and Neobatrachians. To my knowledge, no data of the MOB organization of the earliest diverging frog species, the Archaeobatrachians is available. Nevertheless, the MOB of caudate amphibians are not fused at the midline (Eisthen and Polese, 2007; Eisthen et al., 1994), which strongly suggests that the trait evolved among the earliest frog species between the late Carboniferous period and the early Permian (300-280 Million years ago) (Anderson et al., 2008).

Interestingly, a similarly structured, fused olfactory bulb has been described in passeriform birds (Carl Huber and Crosby, 1929; Corfield et al., 2015; Figure 7B). There is no evidence that this trait might be ancestral in birds however and it is likely that it evolved independently form the MOB of anurans. In contrast to the anuran $\mathrm{MOB}$, there is no overlap of the left and right projections in the Japanese Jungle Crow (Yokosuka et al., 2009a), while we found an overlap of about $40 \%$ in juvenile Xenopus laevis (Figure 7B). Among birds, the passeriform and corvids in particular rely on a set of higher cognitive functions and the relative importance of their sense of smell is unclear (Emery, 2006). Additionally, they have the relatively smallest OBs of all birds and the midline-fusion could possibly be a sign of vestigialization. In the frogs, there is no clear reason to support a similar hypothesis. 
The functional implication of the left-right integrative 'air-bulb' in the anurans is still enigmatic and raises several questions: 1 ) What is the advantage of integrating odorant information from the left and right at the level of the MOB? 2) Why is this particular to the portion of the MOB devoted to the detection of volatile cues? 3) What was the selection pressure in favor of this trait and why is it conserved in all anurans? To address these questions, it would first be necessary to understand, which exact odorants are processed in the $\mathrm{AMOB}$ and particularly the overlap portion of it. RNs in the adult PC system in Xenopus are ciliated (Hansen et al., 1998) and generally express $G_{\text {oolf }}$ (Nakada et al., 2014). OR genes related to the mammalian class II ORs have been found in the adult PC (Freitag et al., 1995, 1998). In rodents, these receptors are responsive to hydrophobic, small volatile chemicals like aldehydes, alcohols or ketones (Saito et al., 2009). However, also some members of the V1R family were found to be expressed in the adult PC (Date-Ito et al., 2008). The expression of TAARs in the adult PC has not been verified yet and the V2Rs expressed in the larval PC progressively vanish from the PC during development (Syed et al., 2017). Especially in the aquatic Xenopus, the question whether the 'air-system' is biologically relevant is completely unaddressed so far. Out of the water, they have been observed in the wild to search for new ponds and lakes thus putatively using their sense of smell for orientation (Kramer, 1933; Measey, 2016; Du Plessis, 1966). Since chemically guided spatial orientation might have favored the integration of odor cues from the left and the right side, this could be a reasonable speculation for the structure of the anuran $\mathrm{dMOB}$. Behavioral experiments coupled to functional imaging or electrophysiology in the $\mathrm{AMOB}$ will be necessary to verify this hypothesis.

\section{The presence of two systems for aquatic olfaction in anurans: the evolution of the VNO}

Since my PhD project focused exclusively on the MOE-MOB axis, I will only briefly discuss the role of the accessory olfactory system. Historically, the VNO was hypothesized to evolve as an adaptation to life on land, since it first appears in terrestrial tetrapods and is lost in many secondarily aquatic tetrapods like whales (Bertmar, 1981). While this could explain the presence of the accessory olfactory system in adult frogs, it does not explain its existence in the tadpoles (Eisthen, 1992). Nevertheless, tadpoles have both a well-developed VNO (Benzekri and Reiss, 2012; Jermakowicz et al., 2004; Jungblut et al., 2012; Figure $6 \mathrm{~A}$ ) and an MOE that is functionally tuned to detect waterborne stimuli (Manzini and Schild, 2010; Figure 6A). The VNO is still present after metamorphosis in adult anurans alongside an additional 'water nose' like the MC or the recessus olfactorius (Eisthen, 1992; Helling, 1938; Figure 6B). The exact functional difference between these 'water noses' and the VNO - which is also water-filled in the frogs - is still unclear.

The VNO of Xenopus has been demonstrated to express only V2Rs (Hagino-Yamagishi et al., 2004), but some more ancient clades of V2Rs are also expressed in the larval MOE and the adult MC (Syed et al., 2013, 2017). The V1Rs instead are only expressed in the main olfactory system (Date-Ito et al., 2008). From the molecular data, it seems like the segregation of vomeronasal and main olfactory system is on a transitional state from the fish-like epithelium to the anatomically segregated mammalian epithelium. In tadpoles of Xenopus laevis, some stimuli like sulfated steroids are concurrently processed by the MOE and the VNO (Sansone et al., 2015) and in salamanders, some olfactory-guided 
behaviors are mediated both through the MOE and the VNO (Park and Propper, 2002; Park et al., 2004). Whether the two pathways are redundant or complementary remains to be verified.

\section{The anuran system between a rodent main and accessory olfactory system?}

The central dogma in vertebrate olfaction concluded from experiments in rodents says that an individual RN (both in the MOE and the VNO) expresses a single allele of a single gene from one of the olfactory receptor gene families (Chess et al., 1994; Rodriguez et al., 1999; Serizawa et al., 2003; Shykind et al., 2004). Axonal projections of all RNs expressing the same allele converge onto two glomeruli per MOB (Mombaerts et al., 1996; Ressler et al., 1994; Vassar et al., 1994) and between 15 and 30 glomeruli in the rodent AOB (Belluscio et al., 1999; Rodriguez et al., 1999). Individual axons in the $\mathrm{MOB}$ were shown to be unbranched prior to entering a single glomerulus, (Klenoff and Greer, 1998). In the AOB, about $20 \%$ of $R N$ axons bifurcated before entering a glomerulus, innervating several glomeruli (Larriva-Sahd, 2008). The accessory system thus shows two levels of signal divergence: on the single RN axon level and the glomerular level, while the main system converges the signal on few glomeruli (Introduction Figure 5).

On the postsynaptic side of the glomeruli, the primary dendritic branches of the projection neurons also show differences in their connectivity in the main and accessory olfactory system. Mitral and tufted cells in the rodent MOB have a single primary dendrite with a single dendritic tuft (Macrides and Schneider, 1982; Nagayama et al., 2014; Orona et al., 1984; Figure 8), while the projection neurons in the AOB get input from multiple glomeruli (Takami and Graziadei, 1991; Yonekura and Yokoi, 2008; Figure 8). The pattern in the rodent $\mathrm{MOB}$ thus presents a labelled line system, while the situation is less clear in the AOB. Morphologically, the $\mathrm{MOB}$ of anuran tadpoles has elements reminiscent of both the rodent $\mathrm{MOB}$ and the rodent $\mathrm{AOB}$ (Figure 8).

On the presynaptic side, I found that $41 \%$ of $\mathrm{RN}$ axons in the tadpole MOB only project to a single glomerulus, while the remaining 59\% innervate at least two glomeruli (Chapter 5, Weiss et al., 2020; Figure 8). This was quite consistent across the four examined anuran species and did not change significantly in postmetamorphotic animals of Rhinella arenarum and Scinax granulatus. The study supports the previous account of bifurcating and multi-glomerular RN projections in the Xenopus laevis MOB (Hassenklöver and Manzini, 2013; Nezlin and Schild, 2005) and AOB (Hassenklöver and Manzini, 2013). On the postsynaptic side, I found a remarkably similar ratio of $56 \%$ uni-tufted vs. $44 \%$ multitufted projection neurons in the $\mathrm{VMOB}$ of postmetamorphotic Xenopus laevis (Chapter 4). This is in accordance with recent descriptions of projection neurons in tadpoles of Xenopus laevis (Offner, 2020) and Xenopus tropicalis (own unpublished results). Similar numbers are also reported for the projection neurons in the MOB of Rana ridibunda (Jiang and Holley, 1992a). I did not conduct any tracings in the $\mathrm{AOB}$ and a population analysis on the ratios of uni- or multi-glomerular projection neurons for frogs are to my knowledge not available. In a salamander species however, projection neurons in the $A O B$ were reported to be almost exclusively multi-glomerular (Laberge, 2008). 
Based on the presented numbers in the anurans, it is tempting to assume that uni-glomerular RN axons and uni-tufted projection neurons form a connectivity pattern similar to the main olfactory system of rodents, while the multi-glomerular pattern is reminiscent of the rodent $A O B$ (Figure $8 \mathrm{~A} ; \mathrm{B}$ ). However, clear experimental evidence is still elusive. It is not clear, whether the uni-glomerular and multiglomerular channels are separate from each other (Figure 8B, labelled line), or whether a single glomerulus can be innervated by a multi-glomerular RN axon branch and a non-bifurcating axon likewise (Figure 8B, alternative wiring). Similarly, the connectivity pattern of the rodent $A O B$ is also not solved yet. It is still unclear, whether individual projection neurons receive information from the same olfactory receptor on their multiple dendritic tufts (homotypic input, Figure 8B, left) or if they integrate between different glomerular input (heterotypic input, Figure 8B, right). Support for the former was given by Del Punta and colleagues (2002), while Wagner and colleagues (2006) showed, that the input was not necessarily from the same receptor allele, but from closely related receptors (selective heterotypic). The functional advantage of receiving homotypic input on two dendrites rather than on a single dendrite is totally unresolved. Integrating heterotypic input could instead facilitate the coincidence detection of several chemicals and odorant blend detection.

To solve the puzzle whether the uni- vs multi-glomerular connectivity in the anurans is related to the mammalian main or accessory system respectively, it will be necessary to find the morphotype of RNs and olfactory receptors linked to the uni- vs multi-glomerular patterns. Since olfactory receptor neurons in the rodent $A O B$ are generally microvillous (Eisthen, 1992; Munger et al., 2009), the multiglomerular projection pattern could theoretically be linked to this morphotype. However, I also found RN axonal bifurcations in the MOE of juvenile Rhinella and Scinax. Since the MOE of juvenile Rhinella consists almost exclusively of an 'air-type' epithelium with only ciliated RNs (Jungblut et al., 2009, 2017), we cannot clearly link the axonal bifurcations to the microvillous RNs. Similarly, RNs innervating the $\mathrm{dMOB}$ in Xenopus have been shown to express $G_{\text {aolf }}$ (Nakada et al., 2014) and are ciliated (Hansen et al., 1998). Still, multi-glomerular projections were observable in the dMOB (Chapter 4). In addition, almost all postsynaptic projection neurons in the $\mathrm{AMOB}$ had dendrites reaching out to multiple glomeruli. These data suggest that also the OR-type expressing, ciliated RNs of the 'air-system' in Xenopus rely at least partially on the multi-glomerular connectivity pattern. Conversely, the VNO of Xenopus tadpoles only consists of microvillous RNs but also occasionally showed RN axons only projecting to a single glomerulus (Hassenklöver and Manzini, 2013). Further studies will be necessary to understand, if the multi-glomerular pattern occurs contextually in RNs expressing different receptors from putatively different receptor gene families. This will also help to understand, if the underlying odor processing is identical to the rodent $A O B$, or if the similarities are just coincidental.

\section{Multi-glomerular wiring patterns as parallel olfactory channels across animals}

Due to the current lack of genetic tools in the anurans, I applied a comparative evolutionary approach to understand if the multi-glomerular projection pattern of individual RN axons might be more relevant in the animal kingdom. Single RN tracings in vertebrate species are quite scarce and the field mostly relies on the rodent data. To test, whether the multi-glomerular RNs might be a shared trait of amphibia in general, I labelled and analyzed axons of individual RNs in the neotenic Axolotl salamander 
(Figure 8A). Multi-glomerular RN projections might be even more common in this species and possibly other urodele amphibians (Chapter 5; Weiss et al., 2020). The axons innervated even more glomeruli than in the anurans and I only found a single uni-glomerular axon. The data from the Axolotl supports the idea that multi-glomerular RN axon projections might be common and possibly conserved among all urodele and anuran amphibians (Figure 8).

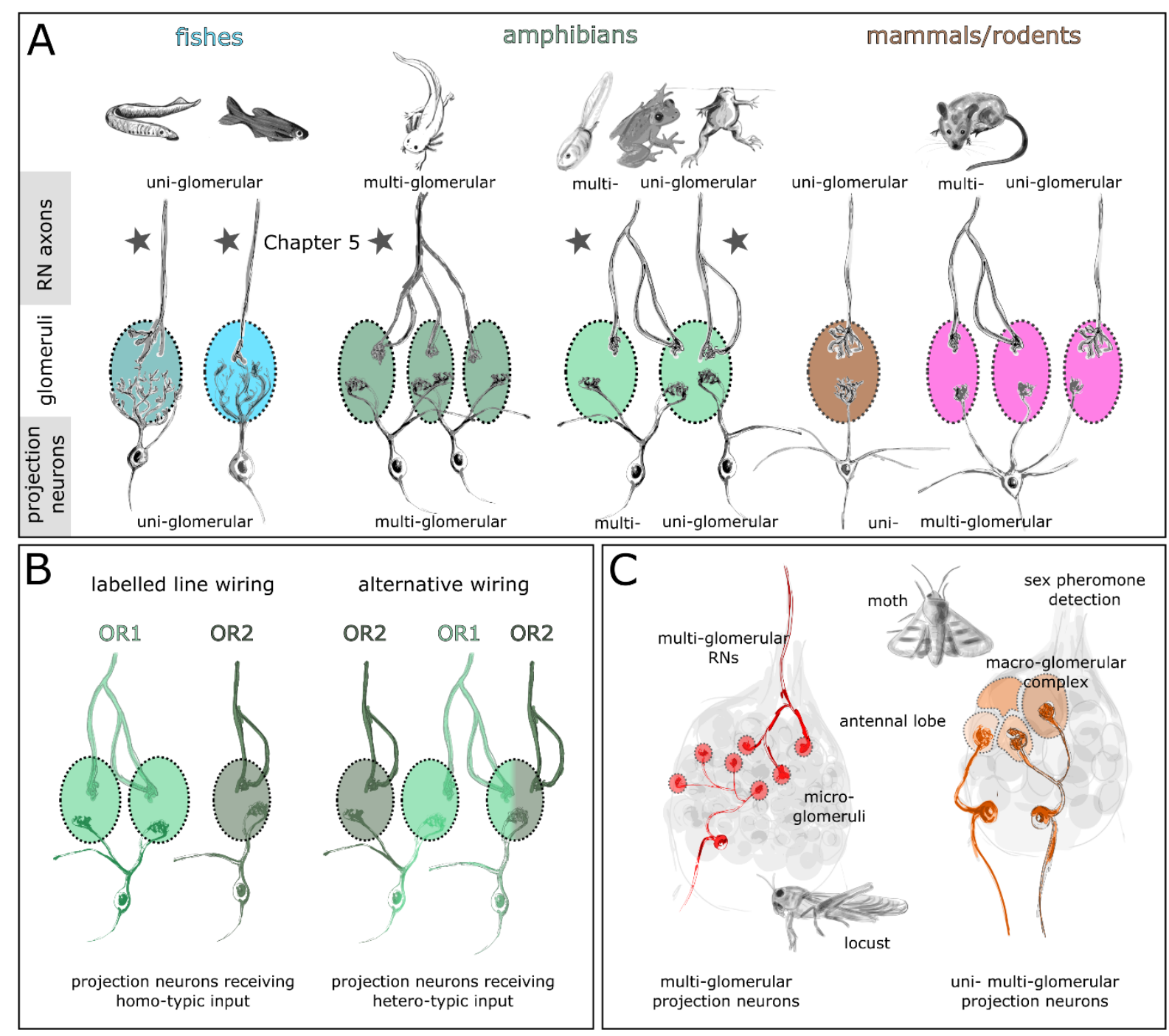

Figure 8 Uni- and multi-glomerular wiring in olfactory systems.

A) The distribution of uni- and multi-glomerular $\mathrm{RN}$ axons and projection neuron dendrites is shown. The amphibians in particular show a mix of both uni-glomerular and multi-glomerular axonal/dendritic patterns (*Chapter 5). B) Two potential wiring logics for the main olfactory system of anurans is depicted. In a labelled line scenario (left), the multi- and uni-glomerular projections are mirrored on the post- and presynaptic side. Projection neurons thus only get input from a particular allele of olfactory receptor (homo-typic input) In the alternative wiring scenario (right), presynaptic signal could be diverged presynaptically and projection neurons could receive hetero-typic input on their multiple dendrites. Similarly, a single glomerulus could contain information from different receptor neuron populations. Other scenarios are possible. C) Multi-glomerular RN axonal projections and multi-tufted projection neurons are also found in the antennal lobe of the locusts (Ignell 
et al., 2001). In the pheromone-detecting macro-glomerular complex of the moth, uni- and multi-glomerular projection neurons constitute different functional processing channels (Lee et al., 2019). The stars indicate results obtained in the course of this thesis.

Contrastingly, individual RN projections in the sea lamprey and the adult zebrafish were almost exclusively uni-glomerular (Weiss et al., 2020). This is again in accordance with the uni-tufted morphology of the projection neurons of the respective species (Fuller et al., 2006; Green et al., 2013; Figue $8 \mathrm{~A}$ ). It is to note however, that these two species of course don't fully account for the variability of fishes and their olfactory systems (Hamdani and Døving, 2007; Olivares and Schmachtenberg, 2019) and because of the small sample size, it is possible that multi-glomerular projections are present in these species as well, although possibly less abundant. The presence of multi-glomerular projection neurons in other ray finned fishes like the goldfish suggests, that the uni-glomerular zebrafish pattern could be a derived trait and that other fish species could also have multi-glomerular RN axon projections. Similarly, projection neurons in the MOB of reptiles are also usually multi-glomerular (Dryer and Graziadei, 1994). More comparative data is necessary to understand which of the two patterns is the ancestral trait in vertebrates, or if they are generally present in parallel.

It is remarkable how similar the cellular composition of the insect and vertebrate system has evolved. In general, RNs in insects also innervate a single glomerulus and individual projection neurons receive information from a single glomerulus (Couto et al., 2005; Hansson and Stensmyr, 2011; Yan et al., 2020). However, several deviations from this norm exist, reminiscent of the pattern I found in the anurans. In orthopteran insects, the antennal lobe consists of about 1000 microglomeruli, which are innervated by multi-glomerular RN projections (Ernst et al., 1983; Ignell et al., 2001; Figure 8C). The morphology of the projection neurons also exhibits the multi-glomerular pattern (Ignell et al., 2001). This trait is hypothesized to be derived and not ancestral in orthopterans, because it gradually developed in this particular clade of insects and is absent in the most basal species (Ignell et al., 2001). In some crustaceans, individual RN projections similarly innervate several glomeruli in the olfactory lobe e.g. in the terrestrial hermit crab (Tuchina et al., 2015) and the spiny lobster (Schmidt et al., 1992) and a single projection neuron often receives input of up to $80 \%$ of all glomeruli (Wachowiak and Ache, 1994). Neither the pre- nor the postsynaptic pattern is functionally explained so far.

A recent study on the functions of projection neurons in the macro-glomerular complex of moths showed that some projection neurons receive input from a single glomerular compartment of the complex while others are multi-glomerular (Lee et al., 2019; Figure 8C). The macro-glomerular complex has been shown to be involved in pheromone detection and Lee and colleagues found evidence, that the multi-glomerular projection neurons are showing different responses to pheromonal blends than their uni-glomerular counterparts and might thus be part of a non-redundant processing channel (Lee et al., 2019). This example shows that both connectivity pattern alongside could be a strategy to extract different aspects of olfactory information. To what extend this is transferrable to the orthopterans, the anurans or the rodent $A O B$ is at this point unclear. 


\section{Summary}

General similarities of the olfactory system from insects to mammals make it tempting to extrapolate from one species to the other. However, the olfactory system is remarkably adaptive and diversified among animal lineages and species occupying different ecological niches. The amphibians are a particularly interesting group of animals, since they are the descendants of the first land living vertebrates and still partially depend on water. In my thesis, I examined adaptive features of the olfactory system of anurans across different distantly related species and in different developmental stages.

- Anuran tadpoles have radiated into many different aquatic microhabitats and have adopted several different lifestyles and feeding habits. Nevertheless, I found that the organization of glomerular clusters in the $\mathrm{MOB}$ is remarkably conserved in anuran larvae, constituting a morphological and possibly functional blueprint for tadpole olfaction.

- Before the onset of metamorphosis, the main olfactory system of Xenopus consists of a sensory epithelium in the PC, which connects to the glomerular clusters in the VMOB. In this thesis, I showed that during metamorphosis, projections to the $\mathrm{VMOB}$ are gradually replaced by cells from the newly formed MC (adult 'water nose') until the metamorphotic climax. Despite the complete rewiring, the $\mathrm{VMOB}$ retains its coarse functional organization and odorant-mediated behavior to waterborne stimuli is still present. In the terrestrial Dendrobates tinctorius, the glomerular clusters of the $\mathrm{VMOB}$ show signs of degeneration, possibly as a sign of less reliance on aquatic olfaction.

- During metamorphosis, cells in the larval PC are completely replaced to form the adult 'air nose'. The new PC neurons project their axons towards a new glomerular projection field in the $\mathrm{dMOB}$. I found that in the $\mathrm{AMOB}, \mathrm{RN}$ projections from the left and right PC show a substantial amount of overlap, and individual glomeruli around the midline receive bilateral input. Postsynaptic projection neurons in the $\mathrm{AMOB}$ extend multiple primary dendrites to multiple glomeruli, putatively integrating bilateral sensory input. It remains to be shown, whether this particular pattern might be behaviorally relevant, e.g. in olfactory-guided spatial orientation.

- Single RN axons in vertebrates are generally believed to be unbranched and only innervate a single glomerulus in the $\mathrm{MOB}$. In my thesis, I show that multi-glomerular projections of individual RNs are conserved among ecologically diverse anurans and are also present in the axolotl. In contrast, the sea lamprey and the zebrafish almost exclusively showed a uniglomerular projection pattern. Axonal bifurcations seem to be an ancestral feature in amphibians, and are possibly more common among vertebrates, forming an alternative odorant processing channel. 


\section{References}

Abuin, L., Bargeton, B., Ulbrich, M.H., Isacoff, E.Y., and Benton, R. (2012). Functional architecture of olfactory ionotropic glutamate receptors. Neuron 69, 44-60.

Adler, E., Hoon, M.A., Mueller, K.L., Chandrashekar, J., Ryba, N.J.P., and Zuker, C.S. (2000). A novel family of mammalian taste receptors. Cell 100, 693-702.

Ahuja, G., and Korsching, S. (2014). Zebrafish olfactory receptor ORA1 recognizes a putative reproductive pheromone. Commun. Integr. Biol. 7:5, e970501.

Ahuja, G., Ivandic, I., Saltürk, M., Oka, Y., Nadler, W., and Korsching, S.I. (2013). Zebrafish crypt neurons project to a single, identified mediodorsal glomerulus. Sci. Rep. 3, 1-9.

Ahuja, G., Nia, S.B., Zapilko, V., Shiriagin, V., Kowatschew, D., Oka, Y., and Korsching, S.I. (2015). Kappe neurons, a novel population of olfactory sensory neurons. Sci. Rep. 4, 1-8.

Ahuja, G., Reichel, V., Kowatschew, D., Syed, A.S., Kotagiri, A.K., Oka, Y., Weth, F., and Korsching, S.I. (2018). Overlapping but distinct topology for zebrafish V2R-like olfactory receptors reminiscent of odorant receptor spatial expression zones. BMC Genomics 19, 1-14.

Alioto, T.S., and Ngai, J. (2006). The repertoire of olfactory C family G protein-coupled receptors in zebrafish: Candidate chemosensory receptors for amino acids. BMC Genomics 7, 1-18.

Altig, R., and Johnston, G.F. (1989). Guilds of anuran larvae: relationships among developmental modes, morphologies, and habitats. Herpetol. Monogr. 3, 81-109.

Altig, R., and McDiarmid, R.W. (1999). Tadpoles: the biology of anuran larvae (Chicago and London: University of Chicago Press).

Anderson, J.S., Reisz, R.R., Scott, D., Fröbisch, N.B., and Sumida, S.S. (2008). A stem batrachian from the Early Permian of Texas and the origin of frogs and salamanders. Nature 453, 515-518.

Andersson, M.N., Schlyter, F., Hill, S.R., and Dekker, T. (2012). What reaches the antenna? How to calibrate odor flux and ligand-receptor affinities. Chem. Senses 37, 403-420.

Araki, I., and Nakamura, H. (1999). Engrailed defines the position of dorsal di-mesencephalic boundary by repressing diencephalic fate. Development 126, 5127-5135.

Araneda, R.C., Kini, A.D., and Firestein, S. (2000). The molecular receptive range of an odorant receptor. Nat. Neurosci. 3, 1248-1255.

Azzouzi, N., Barloy-Hubler, F., and Galibert, F. (2015). Identification and characterization of cichlid TAAR genes and comparison with other teleost TAAR repertoires. BMC Genomics 16, 1-14.

Baier, H., and Korsching, S. (1994). Olfactory glomeruli in the zebrafish form an invariant pattern and are identifiable across animals. J. Neurosci. 14, 219-230.

Bardwell, L. (2005). A walk-through of the yeast mating pheromone response pathway. Peptides 26, 339-350.

Bargmann, C.I. (2006). Comparative chemosensation from receptors to ecology. Nature 444, 295-301. 
Bargmann, C.I., and Mori, I. (1997). Chemotaxis and thermotaxis. In C. Elegans II, D.L. Riddle, D. Blumenthal, B.J. Meyer, and J.R. Priess, eds. (New York: Cold Spring Harbor Laboratory Press), p.

Barnea, G., O'Donnell, S., Mancia, F., Sun, X., Nemes, A., Mandelsohn, M., and Axel, R. (2004). Odorant receptors on axon termini in the brain. Science. 304, 1468.

Barrios, A.W., Sánchez-Quinteiro, P., and Salazar, I. (2014). Dog and mouse: Toward a balanced view of the mammalian olfactory system. Front. Neuroanat. 8, 1-7.

Barth, A.L., Dugas, J.C., and Ngai, J. (1997). Noncoordinate expression of odorant receptor genes tightly linked in the zebrafish genome. Neuron 19, 359-369.

Baxi, K.N., Dorries, K.M., and Eisthen, H.L. (2006). Is the vomeronasal system really specialized for detecting pheromones? Trends Neurosci. 29, 1-7.

Bazáes, A., and Schmachtenberg, O. (2012). Odorant tuning of olfactory crypt cells from juvenile and adult rainbow trout. J. Exp. Biol. 15(Pt 10), 1740-1748.

Bazáes, A., Olivares, J., and Schmachtenberg, O. (2013). Properties, projections, and tuning of teleost olfactory receptor neurons. J. Chem. Ecol. 39, 451-464.

Bear, D.M., Lassance, J.-M., Hoekstra, H.E., and Datta, S.R. (2016). The evolving neural and genetic architecture of vertebrate olfaction. Curr. Biol. 26, R1039-R1049.

Behrens, M., Frank, O., Rawel, H., Ahuja, G., Potting, C., Hofmann, T., Meyerhof, W., and Korsching, S. (2014). ORA1, a Zebrafish olfactory receptor ancestral to all mammalian V1R genes, recognizes 4hydroxyphenylacetic acid , a putative reproductive pheromone. 289, 19778-19788.

Belanger, R.M., Smith, C.M., Corkum, L.D., and Zielinski, B.S. (2003). Morphology and histochemistry of the peripheral olfactory organ in the round goby, Neogobius melanostomus (Teleostei: Gobiidae). J. Morphol. 257, 62-71.

Belluscio, L., Gold, G.H., Nemes, A., and Axel, R. (1998). Mice deficient in G(olf) are anosmic. Neuron $20,69-81$.

Belluscio, L., Koentges, G., Axel, R., and Dulac, C. (1999). A map of pheromone receptor activation in the mammalian brain. Cell 97, 209-220.

Beltz, B.S., Kordas, K., Lee, M.M., Long, J.B., Benton, J.L., and Sandeman, D.C. (2003). Ecological, evolutionary, and functional correlates of sensilla number and glomerular density in the olfactory system of decapod crustaceans. J. Comp. Neurol. 455, 260-269.

Ben-Shaul, Y. (2015). Extracting social information from chemosensory cues: consideration of several scenarios and their functional implications. Front. Neurosci. 9, 1-15.

Benton, R., Vannice, K.S., Gomez-Diaz, C., and Vosshall, L.B. (2009). Variant ionotropic glutamate receptors as chemosensory receptors in Drosophila. Cell 136, 142-162.

Benzekri, N.A., and Reiss, J.O. (2012). Olfactory metamorphosis in the coastal tailed frog Ascaphus truei (Amphibia, Anura, Leiopelmatidae). J. Morphol. 273, 68-87.

Berghard, A., Buck, B., and Cy, G. (1996). Sensory transduction in vomeronasal neurons : evidence for Gao , Gai2 , and adenylyl cyclase II as major components of a pheromone signaling cascade. J. Neurosci. 76, 909-916.

Berry, M.D. (2004). Mammalian central nervous system trace amines. Pharmacologic amphetamines, 
physiologic neuromodulators. J. Neurochem. 90, 257-271.

Bertmar, G. (1981). Evolution of Vomeronasal Organs in Vertebrates. Evolution (N. Y). 35, 359-366.

Bestman, J.E., Ewald, R.C., Chiu, S.-L., and Cline, H.T. (2006). In vivo single-cell electroporation for transfer of DNA and macromolecules. Nat. Protoc. 1, 1267-1272.

Bhatnagar, K.P., and Meisami, E. (1998). Vomeronasal organ in bats and primates: extremes of structural variability and its phylogenetic implications. Microsc. Res. Tech. 43, 465-475.

Bjarnadóttir, T.K., Fredriksson, R., and Schiöth, H.B. (2005). The gene repertoire and the common evolutionary history of glutamate, pheromone (V2R), taste(1) and other related G protein-coupled receptors. Gene 362, 70-84.

Bloom, G. (1954). Studies on the olfactory epithelium of the frog and the toad with the aid of light and electron microscopy. Zeitschrift Für Zellforsch. Und Mikroskopische Anat. 41, 89-100.

Borowsky, B., Adham, N., Jones, K.A., Raddatz, R., Artymyshyn, R., Ogozalek, K.L., Durkin, M.M., Lakhlani, P.P., Bonini, J.A., Pathirana, S., et al. (2001). Trace amines: identification of a family of mammalian G protein-coupled receptors. Proc. Natl. Acad. Sci. U. S. A. 98, 8966-8971.

Boschat, C., Pélofi, C., Randin, O., Roppolo, D., Lüscher, C., Broillet, M.C., and Rodriguez, I. (2002). Pheromone detection mediated by a V1r vomeronasal receptor. Nat. Neurosci. 5, 1261-1262.

Bossuyt, F., and Milinkovitch, M.C. (2000). Convergent adaptive radiations in Madagascan and Asian ranid frogs reveal covariation between larval and adult traits. Proc. Natl. Acad. Sci. U. S. A. 97, 65856590.

Bozza, T., Vassalli, A., Fuss, S., Zhang, J.J., Weiland, B., Pacifico, R., Feinstein, P., and Mombaerts, P. (2009). Mapping of class I and class II odorant receptors to glomerular domains by two distinct types of olfactory sensory neurons in the mouse. Neuron 61, 220-233.

Brand, P., Robertson, H.M., Lin, W., Pothula, R., Klingeman, W.E., Jurat-Fuentes, J.L., and Johnson, B.R. (2018). The origin of the odorant receptor gene family in insects. Elife 7, e38340.

Braubach, O.R., Fine, A., and Croll, R.P. (2012). Distribution and functional organization of glomeruli in the olfactory bulbs of zebrafish (Danio rerio). J. Comp. Neurol. 520, 2317-2339.

Breer, H., Fleischer, J., and Strotmann, J. (2006). The sense of smell: Multiple olfactory subsystems. Cell. Mol. Life Sci. 63, 1465-1475.

Breithaupt, T., and Thiel, M. (2011). Chemical communication in crustaceans (New York: Springer Science+Business Media).

Brennan, P.A., and Zufall, F. (2006). Pheromonal communication in vertebrates. Nature 444, 308-315.

Brinkmann, A., and Schild, D. (2016). One special glomerulus in the olfactory bulb of Xenopus laevis tadpoles integrates a broad range of amino acids and mechanical stimuli. J. Neurosci. 36, 1097810989.

Brown, J.L., Morales, V., and Summers, K. (2008). Divergence in parental care, habitat selection and larval life history between two species of Peruvian poison frogs: An experimental analysis. J. Evol. Biol. 21, 1534-1543.

Brownwell (1998). Glomerular cytoarchitecture in chemosensory systems of arachnids. Ann. N. Y. Acad. Sci. 855, 502-507. 
Brunet, L.J., Gold, G.H., and Ngai, J. (1996). General anosmia caused by a targeted disruption of the mouse olfactory cyclic nucleotide-gated cation channel. Neuron 17, 681-693.

Brykczynska, U., Tzika, A.C., Rodriguez, I., and Milinkovitch, M.C. (2013). Contrasted evolution of the vomeronasal receptor repertoires in mammals and squamate reptiles. Genome Biol. Evol. 5, 389-401.

Buchinger, T.J., Li, W., and Johnson, N.S. (2014). Bile salts as semiochemicals in fish. Chem. Senses 39, 647-654.

Buchinger, T.J., Bussy, U., Li, K., Jia, L., Baker, C.F., Buchinger, E.G., Zhe, Z., Johnson, N.S., and Li, W. (2019). Intra- and interspecific variation in production of bile acids that act as sex pheromones in lampreys. Physiol. Biochem. Zool. 92, 463-472.

Buck, L., and Axel, R. (1991). A novel multigene family may encode odorant receptors: A molecular basis for odor recognition. Cell 65, 175-187.

Bunzow, J.R., Sonders, M.S., Arttamangkul, S., Harrison, L.M., Zhang, G., Quigley, D.I., Darland, T., Suchland, K.L., Pasumamula, S., Kennedy, J.L., et al. (2001). Amphetamine, 3,4methylenedioxymethamphetamine, lysergic acid diethylamide, and metabolites of the catecholamine neurotransmitters are agonists of a rat trace amine receptor. Mol. Pharmacol. 60, 1181-1188.

Burd, G.D. (2000). Development of the olfactory system in the african clawed frog, Xenopus Laevis. In The Biology of Early Influences, Hyson, and Johnson, eds. (New York: Kluwer Academic / Plenum Publishers), pp. 153-170.

Cao, Y., Oh, B.C., and Stryer, L. (1998). Cloning and localization of two multigene receptor families in goldfish olfactory epithelium. Proc. Natl. Acad. Sci. U. S. A. 95, 11987-11992.

Caprio, J., and Byrd, R.P. (1984). Electrophysiological evidence for acidic, basic, and neutral amino acid olfactory receptor sites in the catfish. J. Gen. Physiol. 84, 403-422.

Carey, A.F., Wang, G., Su, C.Y., Zwiebel, L.J., and Carlson, J.R. (2010). Odorant reception in the malaria mosquito Anopheles gambiae. Nature 464, 66-71.

Carl Huber, G., and Crosby, E.C. (1929). The nuclei and fiber paths of the avian diencephalon, with consideration of telencephalic and certain mesencephalic centers and connections. J. Comp. Neurol. $48,1-225$.

Catania, K.C. (2013). Stereo and serial sniffing guide navigation to an odour source in a mammal. Nat. Commun. 4, 1441-1448.

Chamero, P., Katsoulidou, V., Hendrix, P., Bufe, B., Roberts, R., Matsunami, H., Abramowitz, J., Birnbaumer, L., Zufall, F., and Leinders-Zufall, T. (2011). G protein Gao is essential for vomeronasal function and aggressive behavior in mice. Proc. Natl. Acad. Sci. U. S. A. 108, 12898-12903.

Chang, S., Chung-Davidson, Y.-W., Libants, S. V, Nanlohy, K.G., Kiupel, M., Brown, C., and Li, W. (2013). The sea lamprey has a primordial accessory olfactory system. BMC Evol. Biol. 13, 172.

Chase, R., and Tolloczko, B. (1986). Synaptic glomeruli in the olfactory system of a snail, Achatina fulica. Cell Tissue Res. 246, 567-573.

Chen, C., Smye, S.W., Robinson, M.P., and Evans, J.A. (2006). Membrane electroporation theories: A review. Med. Biol. Eng. Comput. 44, 5-14.

Chess, A., Simon, I., Cedar, H., and Axel, R. (1994). Allelic inactivation regulates olfactory receptor gene 
expression. Cell 78, 823-834.

Churcher, A.M., and Taylor, J.S. (2009). Amphioxus (Branchiostoma floridae) has orthologs of vertebrate odorant receptors. BMC Evol. Biol. 9, 1-10.

Churcher, A.M., and Taylor, J.S. (2010). The antiquity of chordate odorant receptors is revealed by the discovery of orthologs in the cnidarian Nematostella vectensis. Genome Biol. Evol. 3, 36-43.

Cline, H., and Haas, K. (2008). The regulation of dendritic arbor development and plasticity by glutamatergic synaptic input: a review of the synaptotrophic hypothesis. J. Physiol. 586, 1509-1517.

Clyne, P.J., Warr, C.G., Freeman, M.R., Lessing, D., Kim, J., and Carlson, J.R. (1999). A novel family of divergent seven-transmembrane proteins: Candidate odorant receptors in Drosophila. Neuron 22, 327-338.

Cole, T.B., and Stacey, N.E. (2006). Olfactory responses to steroids in an African mouth-brooding cichlid, Haplochromis burtoni (Günther). J. Fish Biol. 68, 661-680.

Cong, X., Zheng, Q., Ren, W., Chéron, J.B., Fiorucci, S., Wen, T., Zhang, C., Yu, H., Golebiowski, J., and $\mathrm{Yu}, \mathrm{Y}$. (2019). Zebrafish olfactory receptors ORAs differentially detect bile acids and bile salts. J. Biol. Chem. 294, 6762-6771.

Corey, E.A., Bobkov, Y., Ukhanov, K., and Ache, B.W. (2013). Ionotropic crustacean olfactory receptors. PLoS One 8, e60551.

Corfield, J.R., Price, K., Iwaniuk, A.N., Gutiérrez-Ibáñez, C., Birkhead, T., and Wylie, D.R. (2015). Diversity in olfactory bulb size in birds reflects allometry, ecology, and phylogeny. Front. Neuroanat. 9, 1-16.

Couto, A., Alenius, M., and Dickson, B.J. (2005). Molecular, anatomical, and functional organization of the Drosophila olfactory system. Curr. Biol. 15, 1535-1547.

Croset, V., Rytz, R., Cummins, S.F., Budd, A., Brawand, D., Kaessmann, H., Gibson, T.J., and Benton, R. (2010). Ancient protostome origin of chemosensory ionotropic glutamate receptors and the evolution of insect taste and olfaction. PLoS Genet. 6, e1001064.

Cummins, S.F., Erpenbeck, D., Zou, Z., Claudianos, C., Moroz, L.L., Nagle, G.T., and Degnan, B.M. (2009). Candidate chemoreceptor subfamilies differentially expressed in the chemosensory organs of the mollusc Aplysia. BMC Biol. 7, 1-20.

Daghfous, G., Green, W.W., Alford, S.T., Zielinski, B.S., and Dubuc, R. (2016). Sensory activation of command cells for locomotion and modulatory mechanisms: lessons from lampreys. Front. Neural Circuits 10, 1-17.

Dalton, R.P., and Lomvardas, S. (2015). Chemosensory receptor specificity and regulation. Annu. Rev. Neurosci. 38, 331-349.

Date-Ito, A., Ohara, H., Ichikawa, M., Mori, Y., and Hagino-Yamagishi, K. (2008). Xenopus V1R vomeronasal receptor family is expressed in the main olfactory system. Chem. Senses 33, 339-346.

Dawley, E.M. (1998). Species, sex, and seasonal differences in VNO size. Microsc. Res. Tech. 41, 506518.

DeMaria, S., Berke, A.P., Van Name, E., Heravian, A., Ferreira, T., and Ngai, J. (2013). Role of a ubiquitously expressed receptor in the vertebrate olfactory system. J. Neurosci. 33, 15235-15247.

Derby, C.D., Kozma, M.T., Senatore, A., and Schmidt, M. (2016). Molecular mechanisms of reception 
and perireception in crustacean chemoreception: A comparative review. Chem. Senses 41, 381-398.

Derjean, D., Moussaddy, A., Atallah, E., St-Pierre, M., Auclair, F., Chang, S., Ren, X., Zielinski, B., and Dubuc, R. (2010). A novel neural substrate for the transformation of olfactory inputs into motor output. PLoS Biol. 8, e1000567.

Dieris, M., Ahuja, G., Krishna, V., and Korsching, S.I. (2017). A single identified glomerulus in the zebrafish olfactory bulb carries the high-Affinity response to death-Associated odor cadaverine. Sci. Rep. 7, 40892.

Dietschi, Q., Assens, A., Challet, L., Carleton, A., and Rodriguez, I. (2013). Convergence of FPR-rs3expressing neurons in the mouse accessory olfactory bulb. Mol. Cell. Neurosci. 56, 140-147.

Dittrich, K., Kuttler, J., Hassenklöver, T., and Manzini, I. (2016). Metamorphic remodeling of the olfactory organ of the African clawed frog, Xenopus laevis. J. Comp. Neurol. 524, 986-998.

Dohlman, H.G. (2002). G Proteins and Pheromone Signaling. Annu. Rev. Physiol. 64, 129-152.

Dryer, L., and Graziadei, P.P.C. (1993). A pilot study on morphological compartmentalization and heterogeneity in the elasmobranch olfactory bulb. Anat. Embryol. (Berl). 188, 41-51.

Dryer, L., and Graziadei, P.P.C. (1994). Mitral cell dendrites: a comparative approach. Anat. Embryol. (Berl). 189, 91-106.

Duellman, W.E., and Trueb, L. (1994). Biology of amphibians (Baltimore: John Hopkins University Press).

Dugas, J.C., and Ngai, J. (2001). Analysis and characterization of an odorant receptor gene cluster in the zebrafish genome. Genomics 71, 53-65.

Dulac, C., and Axel, R. (1995). A novel family of genes encoding putative pheromone receptors in mammals. Cell 83, 195-206.

Dynes, J.L., and Ngai, J. (1998). Pathfinding of olfactory neuron axons to stereotyped glomerular targets revealed by dynamic imaging in living zebrafish embryos. Neuron 20,1081-1091.

Ebbesson, S.O.E.E., Bazer, G.T., and Jane, J.A. (1986). Some primary olfactory axons project to the contralateral olfactory bulb in Xenopus laevis. Neurosci. Lett. 65, 234-238.

Eisthen, H.L. (1992). Phylogeny of the vomeronasal system and of receptor cell types in the olfactory and vomeronasal epithelia of vertebrates. Microsc. Res. Tech. 23, 1-21.

Eisthen, H.L. (1997). Evolution of vertebrate olfactory systems. Brain. Behav. Evol. 50, 222-233.

Eisthen, H.L. (2000). Presence of the vomeronasal system in aquatic salamanders. Philos. Trans. R. Soc. B Biol. Sci. 355, 1209-1213.

Eisthen, H.L., and Polese, G. (2007). Components of the vertebrate olfactory system. 390, 355-406.

Eisthen, H.L., Sengelaub, D.R., Schroeder, D.M., Alberts, J.R., Eisthen, H.L., Sengelaub, D.R., Schroeder, D.M., and Alberts, J.R. (1994). Anatomy and forebrain projections of the olfactory and vomeronasal organs in axolotls (ambystoma mexicanum). Brain. Behav. Evol. 44, 108-124.

Emery, N.J. (2006). Cognitive ornithology: The evolution of avian intelligence. Philos. Trans. R. Soc. B Biol. Sci. 361, 23-43. 
Ernst, K.D., Boeckh, J., and Boeckh, V. (1977). A neuroanatomical study on the organization of the central antennal pathways in insects II. Deutocerebral connections in Locusta migratoria and Periplaneta americana. Cell Tissue Res. 176, 285-308.

Ernst, K.D.D., Boeckh, J., and Boeckh, V. (1983). A neuroanatomical study on the organization of the central antennal pathways in insects III Neuroanatomical characterization of physiologically defined response types of deutocerebral neurons in Periplaneta americana. Cell Tissue Res. 229, 1-22.

Eyun, S. il (2019). Accelerated pseudogenization of trace amine-associated receptor genes in primates. Genes, Brain Behav. 18, 1-12.

Eyun, S. II, Soh, H.Y., Posavi, M., Munro, J.B., Hughes, D.S.T., Murali, S.C., Qu, J., Dugan, S., Lee, S.L., Chao, H., et al. (2017). Evolutionary history of chemosensory-related gene families across the arthropoda. Mol. Biol. Evol. 34, 1838-1862.

Falk, J., Drinjakovic, J., Leung, K., Dwivedy, A., Regan, A.G., Piper, M., and Holt, C.E. (2007). Electroporation of cDNA/morpholinos to targeted areas of embryonic CNS in Xenopus. BMC Dev. Biol. 7, 107.

Feinstein, P., and Mombaerts, P. (2004). A contextual model for axonal sorting into glomeruli in the mouse olfactory system. Cell 117, 817-831.

Ferrando, S., and Gallus, L. (2013). Is the olfactory system of cartilaginous fishes a vomeronasal system? Front. Neuroanat. 7, 1-4.

Ferrando, S., Gambardella, C., Ravera, S., Bottero, S., Ferrando, T., Gallus, L., Manno, V., Salati, A.P., Ramoino, P., and Tagliafierro, G. (2009). Immunolocalization of G-protein alpha subunits in the olfactory system of the cartilaginous fish Scyliorhinus canicula. Anat. Rec. 292, 1771-1779.

Ferrando, S., Gallus, L., Amaroli, A., Gambardella, C., Waryani, B., Di Blasi, D., and Vacchi, M. (2017). Gross anatomy and histology of the olfactory rosette of the shark Heptranchias perlo. Zoology 122, 27-37.

Ferreira, T., Wilson, S.R., Choi, Y.G., Risso, D., Dudoit, S., Speed, T.P., and Ngai, J. (2014). Silencing of odorant receptor gene expression by $\mathrm{G}$ protein $\beta \gamma$ signaling ensures the expression of one odorant receptor per olfactory. Neuron $81,847-859$.

Ferrero, D.M., Lemon, J.K., Fluegge, D., Pashkovski, S.L., Korzan, W.J., Datta, S.R., Spehr, M., Fendt, M., and Liberles, S.D. (2011). Detection and avoidance of a carnivore odor by prey. Proc. Natl. Acad. Sci. U. S. A. $108,11235-11240$.

Finlay, B.L., and Darlington, R.B. (1995). Linked regularities in the development and evolution of mammalian brains. Science. 268, 1578-1584.

Firestein, S. (2001). How the olfactory system makes sense of scents. Nature 413, 211-218.

Fischer, E.K., Alvarez, H., Lagerstrom, K.M., Petrillo, R., Ellis, G., and O'Connell, L.A. (2020). Neural correlates of winning and losing fights in poison frog tadpoles. Physiol. Behav. 223, 112973.

Fishilevich, E., and Vosshall, L.B. (2005). Genetic and functional subdivision of the Drosophila antennal lobe. Curr. Biol. 15, 1548-1553.

Forester, D.C., and Wisnieski, A. (1991). The significance of airborne olfactory cues to the recognition of home area by the dart-poison frog Dendrobates pumilio. J. Herpetol. 25, 502-504. 
Föske, H. (1934). Das Geruchsorgan von Xenopus laevis. Z. Anat. Entwicklungsgesch. 103, 519-550.

Franceschini, V., Giorgi, P.P.P., and Ciani, F. (1992). Primary olfactory terminations in the forebrain of amphibia: a comparative study with soybean agglutinin. J. Hirnforsch. 33, 627-635.

Fredriksson, R., Schio, H.B., Krishnan, A., and Sa, M. (2012). The Origin of GPCRs : identification of mammalian like rhodopsin, adhesion, glutamate and frizzled GPCRs in fungi. PLoS One 7, e29817.

Freitag, J., Krieger, J., Strotmann, J., and Breer, H. (1995). Two classes of olfactory receptors in Xenopus laevis. Neuron 15, 1383-1392.

Freitag, J., Ludwig, G., Andreini, I., Roessler, P., Breer, H., Rössler, P., Breer, H., Roessler, P., and Breer, H. (1998). Olfactory receptors in aquatic and terrestrial vertebrates. J. Comp. Physiol. A-Sensory Neural Behav. Physiol. 183, 635-650.

Freitag, J., Beck, A., Ludwig, G., Von Buchholtz, L., and Breer, H. (1999). On the origin of the olfactory receptor family: Receptor genes of the jawless fish (Lampetra fluviatilis). Gene.

Friedrich, R.W., and Korsching, S.I. (1998). Representations in the olfactory bulb revealed using a voltage-sensitive axon tracer. J. Neurosci. 18, 9977-9988.

Friedrich, J., Zhou, P., and Paninski, L. (2017). Fast online deconvolution of calcium imaging data. PLoS Comput. Biol. 13, e1005423.

Frontini, A., Zaidi, A.U., Hua, H., Wolak, T.P., Greer, C.A., Kafitz, K.W., Li, W., and Zielinski, B.S. (2003). Glomerular territories in the olfactory bulb from the larval stage of the sea lamprey Petromyzon marinus. J. Comp. Neurol. 465, 27-37.

Fuller, C.L., Yettaw, H.K., and Byrd, C.A. (2006). Mitral cells in the olfactory bulb of adult zebrafish (Danio rerio): Morphology and distribution. J. Comp. Neurol. 499, 218-230.

Gao, Q., and Chess, A. (1999). Identification of candidate Drosophila olfactory receptors from genomic DNA sequence. Genomics 60, 31-39.

Garcia-Verdugo, J.M., Llahi, S., Farinas, I., and Martin, V. (1986). Laminar organization of the main olfactory bulb of Podarcis hispanica: An electron microscopic and Golgi study. J. Hirnforsch. 27, 87100.

Garwood, R.J., and Edgecombe, G.D. (2011). Early Terrestrial Animals, Evolution, and Uncertainty. Evol. Educ. Outreach 4, 489-501.

Gaudin, A., and Gascuel, J. (2005). 3D atlas describing the ontogenic evolution of the primary olfactory projections in the olfactory bulb of Xenopus laevis. J. Comp. Neurol. 489, 403-424.

Getchell, M.L., and Getchell, T. V. (1992). Fine structural aspects of secretion and extrinsic innervation in the olfactory mucosa. Microsc. Res. Tech. 23, 111-127.

Giovannucci, A., Friedrich, J., Gunn, P., Kalfon, J., Brown, B.L., Koay, S.A., Taxidis, J., Najafi, F., Gauthier, J.J.L., Zhou, P., et al. (2019). CalmAn an open source tool for scalable calcium imaging data analysis. Elife 8, e38173.

Saint Girons, H., and Zylberberg, I. (1992). Histologie comparée des glandes céphaliques exocrines et des fosses nasales des Lissamphibia. Ann. Mus. Hist. Nat. 13, 59-82.

Gliem, S., Syed, A.S., Sansone, A., Kludt, E., Tantalaki, E., Hassenklöver, T., Korsching, S.I., and Manzini, I. (2013). Bimodal processing of olfactory information in an amphibian nose: Odor responses segregate 
into a medial and a lateral stream. Cell. Mol. Life Sci. 70, 1965-1984.

Gloriam, D.E.I., Bjarnadóttir, T.K., Yan, Y., Postlethwait, J.H., Schiöth, H.B., and Fredriksson, R. (2005). The repertoire of trace amine G-protein-coupled receptors : large expansion in zebra W sh. 35, 470482.

Glusman, G., Bahar, A., Sharon, D., Pilpel, Y., White, J., and Lancet, D. (2000). The olfactory receptor gene superfamily: Data mining, classification, and nomenclature. Mamm. Genome 11, 1016-1023.

Gomez-Diaz, C., Martin, F., Garcia-Fernandez, J.M., and Alcorta, E. (2018). The two main olfactory receptor families in drosophila, ORs and IRs: A comparative approach. Front. Cell. Neurosci. 12, 253.

Gonzalez-Voyer, A., Winberg, S., and Kolm, N. (2009). Brain structure evolution in a basal vertebrate clade: Evidence from phylogenetic comparative analysis of cichlid fishes. BMC Evol. Biol. 9, 238.

González, A. (2010). Lungfishes, like tetrapods, possess a vomeronasal system. Front. Neuroanat. 4, 130.

Gosner, K.L. (1960). A simplified table for staging anuran embryos and larvae with notes on identification. Herpetologica 16, 183-190.

Graziadei, P.P.C., and Bannister, L.H. (1967). Some observations on the fine structure of the olfactory epithelium in the domestic duck. Zeitschrift Für Zellforsch. 80, 220-228.

Green, W.W., Basilious, A., Dubuc, R., and Zielinski, B.S. (2013). The neuroanatomical organization of projection neurons associated with different olfactory bulb pathways in the sea lamprey, Petromyzon marinus. PLoS One 8, e69525.

Green, W.W., Boyes, K., McFadden, C., Daghfous, G., Auclair, F., Zhang, H., Li, W., Dubuc, R.R.R.R., and Zielinski, B.S. (2017). Odorant organization in the olfactory bulb of the sea lamprey. J. Exp. Biol. 220, 1350-1359.

Greer, P.L., Bear, D.M., Lassance, J.M., Bloom, M.L., Tsukahara, T., Pashkovski, S.L., Masuda, F.K., Nowlan, A.C., Kirchner, R., Hoekstra, H.E., et al. (2016). A family of non-GPCR chemosensors defines an alternative logic for mammalian olfaction. Cell 165, 1734-1748.

Grosmaitre, X., Fuss, S.H., Lee, A.C., Adipietro, K.A., Matsunami, H., Mombaerts, P., and Ma, M. (2009). SR1, a mouse odorant receptor with an unusually broad response profile. J. Neurosci. 29, 1454514552.

Grubb, J.C. (1975). Olfactory orientation in southern leopard frogs, Rana utricularia. Herpetologica 31, 219-221.

Grüneberg, H. (1973). A ganglion probably belonging to the N. terminalis system in the nasal mucosa of the mouse. Z. Anat. Entwicklungsgesch. 140, 39-52.

Grus, W.E., and Zhang, J. (2009). Origin of the genetic components of the vomeronasal system in the common ancestor of all extant vertebrates. Mol. Biol. Evol. 26, 407-419.

Grus, W.E., Shi, P., and Zhang, J. (2007). Largest vertebrate vomeronasal type 1 receptor gene repertoire in the semiaquatic platypus. Mol. Biol. Evol. 24, 2153-2157.

Haas, K., Sin, W.C., Javaherian, A., Li, Z., and Cline, H.T. (2001). Single-cell electroporation for gene transfer in vivo. Neuron 29, 583-591.

Haas, K., Jensen, K., Sin, W.C., Foa, L., and Cline, H.T. (2002). Targeted electroporation in Xenopus 
tadpoles in vivo -from single cells to the entire brain. Differentiation 70, 148-154.

Haga, S., Hattori, T., Sato, T., Sato, K., Matsuda, S., Kobayakawa, R., Sakano, H., Yoshihara, Y., Kikusui, T., and Touhara, K. (2010). The male mouse pheromone ESP1 enhances female sexual receptive behaviour through a specific vomeronasal receptor. Nature 466, 118-122.

Hagino-Yamagishi, K., and Nakazawa, H. (2011). Involvement of $\mathrm{G} \alpha$ olf-expressing neurons in the vomeronasal system of Bufo japonicus. J. Comp. Neurol. 519, 3189-3201.

Hagino-Yamagishi, K., Moriya, K., Kubo, H., Wakabayashi, Y., Isobe, N., Saito, S., Ichikawa, M., and Yazaki, K. (2004). Expression of vomeronasal receptor genes in Xenopus laevis. J. Comp. Neurol. 472, 246-256.

Hallem, E.A., and Carlson, J.R. (2006). Coding of Odors by a Receptor Repertoire. Cell 125, 143-160.

Hallem, E.A., Ho, M.G., and Carlson, J.R. (2004). The molecular basis of odor coding in the Drosophila antenna. Cell 117, 965-979.

Halpern, M., Shapiro, L.S., and Jia, C. (1995). Differential localization of G proteins in the opossum vomeronasal system. $677,157-161$.

Hamdani, E.H. (2001a). Is feeding behaviour in Crucian Carp mediated by the lateral olfactory tract? Chem. Senses 26, 1133-1138.

Hamdani, E.H. (2001b). Projection of sensory neurons with microvilli to the lateral olfactory tract indicates their participation in feeding behaviour in Crucian Carp. Chem. Senses 26, 1139-1144.

Hamdani, E.H. (2002). The Alarm Reaction in Crucian Carp is Mediated by Olfactory Neurons with Long Dendrites. Chem. Senses 27, 395-398.

Hamdani, E.H., and Døving, K.B. (2006). Specific projection of the sensory crypt cells in the olfactory system in crucian carp, Carassius carassius. Chem. Senses 31, 63-67.

Hamdani, E.H., and Døving, K.B. (2007). The functional organization of the fish olfactory system. Prog. Neurobiol. 82, 80-86.

Hansen, A. (2007). Olfactory and solitary chemosensory cells: Two different chemosensory systems in the nasal cavity of the American alligator, Alligator mississippiensis. BMC Neurosci. 8, 1-10.

Hansen, A., and Finger, T.E. (2000). Phyletic distribution of crypt-type olfactory receptor neurons in fishes. Brain. Behav. Evol. 55, 100-110.

Hansen, A., and Zeiske, E. (1998). The peripheral olfactory organ of the zebrafish, Danio rerio: an ultrastructural study. Chem. Senses 23, 39-48.

Hansen, A., and Zielinski, B.S. (2005). Diversity in the olfactory epithelium of bony fishes: Development, lamellar arrangement, sensory neuron cell types and transduction components. J. Neurocytol. 34, 183208.

Hansen, A., Reiss, J.O., Gentry, C.L., and Burd, G.D. (1998). Ultrastructure of the olfactory organ in the clawed frog, Xenopus laevis, during larval development and metamorphosis. J. Comp. Neurol. 288, 273-288.

Hansen, A., Rolen, S.H., Anderson, K., Morita, Y., Caprio, J., and Finger, T.E. (2003). Correlation between olfactory receptor cell type and function in the channel catfish. J. Neurosci. 23, 9328 -9339. 
Hansen, A., Anderson, K.T., and Finger, T.E. (2004). Differential distribution of olfactory receptor neurons in goldfish: Structural and molecular correlates. J. Comp. Neurol. 477, 347-359.

Hansson, B.S.S., and Stensmyr, M.C.C. (2011). Evolution of insect olfaction. Neuron 72, 698-711.

Hansson, B., Ljungberg, H., Hallberg, E., and Lofstedt, C. (1992). Functional specialization of olfactory glomeruli in a moth. Science. 256, 1313-1315.

Hara, T.J., and Zhang, C. (1997). Topographic bulbar projections and dual neural pathways of the primary olfactory neurons in salmonid fishes. Neuroscience 82, 301-313.

Hart, A.C., and Chao, M.Y. (2010). From odors to behaviors in caenorhabditis elegans. In The Neurobiology of Olfaction, A. Menini, ed. (Boca Raton, FL: CRC Press/Taylor \& Francis), p.

Harzsch, S., and Krieger, J. (2018). Crustacean olfactory systems: A comparative review and a crustacean perspective on olfaction in insects. Prog. Neurobiol. 161, 23-60.

Hashiguchi, Y., and Nishida, M. (2007). Evolution of trace amine - associated receptor ( TAAR ) gene family in vertebrates : lineage-specific expansions and degradations of a second class of vertebrate chemosensory receptors expressed in the olfactory epithelium. Mol. Biol. Evol. 24, 2099-2107.

Hassenklöver, T., and Manzini, I. (2013). Olfactory wiring logic in amphibians challenges the basic assumptions of the unbranched axon concept. J. Neurosci. 33, 17247-17252.

Hassenklöver, T., and Manzini, I. (2014). The olfactory system as a model to study axonal growth patterns and orphology in vivo. J. Vis. Exp. e52143.

Hatakana, T., and Matsuzaki, O. (1993). Odor responses of the vomeronasal system in Reeve's turtle, Geoclemys reevesii. Brain. Behav. Evol. 41, 183-186.

Heinicke, M.P., Duellman, W.E., Trueb, L., Means, D.B., Macculloch, R.D., and Hedges, S.B. (2009). A new frog family (Anura: Terrarana) from South America and an expanded direct-developing clade revealed by molecular phylogeny. Zootaxa 2211, 1-35.

Helling, H. (1938). Das Geruchsorgan der Anuren, vergleichend-morphologisch betrachtet. Z. Anat. Entwicklungsgesch. 108, 587-643.

Herrada, G., and Dulac, C. (1997). A novel family of putative pheromone receptors in mammals with a topographically organized and sexually dimorphic distribution. Cell 90, 763-773.

Herrick, C.J. (1910). The morphology of the forebrain in amphibia and reptilia. J. Comp. Neurol. Psychol. 20, 413-547.

Herrick, C.J. (1924). The amphibian forebrain. II. The olfactory bulb of Amblystoma. J. Comp. Neurol. $37,55-69$.

Hewapathirane, D.S., and Haas, K. (2008). Single Cell Electroporation in vivo within the Intact Developing Brain. J. Vis. Exp. 17, e705.

Higgs, D.M., and Burd, G.D. (2001). Neuronal turnover in the Xenopus laevis olfactory epithelium during metamorphosis. J. Comp. Neurol. 433, 124-130.

Hildebrand, J.G., and Shepherd, G.M. (1997). Mechanisms of olfactory discrimination: converging evidence for common principles across phyla. Annu. Rev. Neurosci. 20, 595-631.

Ho, S.Y., and Mittal, G.S. (1996). Electroporation of cell membranes: a review. Crit. Rev. Biotechnol. 16, 
349-362.

Hoffman, H.H. (1963). The olfactory bulb, accessory bulb, and hemisphere of some anurans. J. Comp. Neurol. 120, 317-368.

Hofmann, M.H., and Meyer, D.L. (1991). Functional subdivisions of the olfactory system correlate with lectin-binding properties in Xenopus. Brain Res. 564, 344-347.

Homberg, U., Montague, R.A., and Hildebrand, J.G. (1988). Anatomy of antenno-cerebral pathways in the brain of the sphinx moth Manduca sexta. Cell Tissue Res. 254, 255-281.

Houck, L.D. (2009). Pheromone communication in amphibians and reptiles. Annu. Rev. Physiol. 71, 161-176.

Hovis, K.R., Padmanabhan, K., and Urban, N.N. (2010). A simple method of in vitro electroporation allows visualization, recording, and calcium imaging of local neuronal circuits. J. Neurosci. Methods 191, 1-10.

Howard, L.E., Holmes, W.M., Ferrando, S., Maclaine, J.S., Kelsh, R.N., Ramsey, A., Abel, R.L., and Cox, J.P.L. (2013). Functional nasal morphology of chimaerid fishes. J. Morphol.

Hu, J., Zhong, C., Ding, C., Chi, Q., Walz, A., Mombaerts, P., Matsunami, H., and Luo, M. (2007). Detection of near-atmospheric concentrations of $\mathrm{CO} 2$ by an olfactory subsystem in the mouse. Science . 317, 953-957.

Huang, L.K., and Wang, M.J.J. (1995). Image thresholding by minimizing the measures of fuzziness. Pattern Recognit. 28, 41-51.

Huang, G.Z., Zhang, J.J., Wang, D., Mason, R.T., and Halpern, M. (2006). Female snake sex pheromone induces membrane responses in vomeronasal sensory neurons of male snakes. Chem. Senses 31, 521529.

Hussain, A., Saraiva, L.R., and Korsching, S.I. (2009). Positive Darwinian selection and the birth of an olfactory receptor clade in teleosts. 106, 4313-4318.

Hussain, A., Saraiva, L.R., Ferrero, D.M., Ahuja, G., Krishna, V.S., Liberles, S.D., and Korsching, S.I. (2013). High-affinity olfactory receptor for the death-associated odor cadaverine. Proc. Natl. Acad. Sci. U. S. A. $110,19579-19584$.

Ignell, R., Anton, S., and Hansson, B.S. (2001). The antennal lobe of orthoptera - Anatomy and evolution. Brain. Behav. Evol. 57, 1-17.

Ishii, T., and Mombaerts, P. (2008). Expression of nonclassical class I major histocompatibility genes defines a tripartite organization of the mouse vomeronasal system. J. Neurosci. 28, 2332-2341.

Isogai, Y., Si, S., Pont-Lezica, L., Tan, T., Kapoor, V., Murthy, V.N., and Dulac, C. (2011). Molecular organization of vomeronasal chemoreception. Nature 478, 241-245.

Iwahori, N., Kiyota, E., and Nakamura, K. (1987). A Golgi study on the olfactory bulb in the lamprey, Lampetra japonica. Neurosci. Res. 5, 126-139.

Iwahori, N., Nakamura, K., and Mameya, C. (1989). A Golgi study on the main olfactory bulb in the snake Elaphe quadrivirgata. Neurosci. Res.

Iwata, T., Nakada, T., Toyoda, F., Yada, T., Shioda, S., and Kikuyama, S. (2013). Responsiveness of vomeronasal cells to a newt peptide pheromone, sodefrin as monitored by changes of intracellular 
calcium concentrations. Peptides 45, 15-21.

Jacobson, L. (1811). Description anatomique d'un organe observe dans les mammifieres. Ann. Mus. Hist. Nat. 18, 412-424.

Jerison, H.J. (1973). Evolution of the brain and intelligence. (New York: Academic Press).

Jermakowicz, W.J., Dorsey, D.A., Brown, A.L., Wojciechowski, K., Giscombe, C.L., Graves, B.M., Summers, C.H., and Ten Eyck, G.R. (2004). Development of the nasal chemosensory organs in two terrestrial anurans: The directly developing frog, Eleutherodactylus coqui (Anura: Leptodactylidae), and the metamorphosing toad, Bufo americanus (Anura: Bufonidae). J. Morphol. 261, 225-248.

Jia, C., and Halpern, M. (1996). Subclasses of vomeronasal receptor neurons: Differential expression of G proteins ( $G i \alpha 2$ and $G o \alpha$ ) and segregated projections to the accessory olfactory bulb. Brain Res. 719, 117-128.

Jiang, T., and Holley, A. (1992a). Morphological variations among output neurons of the olfactory bulb in the frog (Rana ridibunda). J. Comp. Neurol. 320, 86-96.

Jiang, T., and Holley, A. (1992b). Some properties of receptive fields of olfactory mitral/tufted cells in the frog. J. Neurophysiol. 68, 726-733.

Jiang, H., Du, K., Gan, X., Yang, L., and He, S. (2019). Massive loss of olfactory receptors but not trace amine-associated receptors in the world's deepest-living fish (Pseudoliparis swirei). Genes (Basel). 10, 910.

Jiang, X.C., Inouchi, J., Wang, D., and Halpern, M. (1990). Purification and characterization of a chemoattractant from electric shock-induced earthworm secretion, its receptor binding, and signal transduction through the vomeronasal system of garter snakes. J. Biol. Chem. 265, 8736-8744.

Johansson, M.L., and Banks, M. (2010). Genetic patterns of demography and diversity in eastern north pacific rockfishes (genus Sebastes).

Johansson, M.L., and Banks, M.A. (2011). Olfactory receptor related to class A, Type 2 (VIr-Like Ora2) genes are conserved between distantly related rockfishes (Genus sebastes). J. Hered. 102, 113-117.

Johnson, M.A., Tsai, L., Roy, D.S., Valenzuela, D.H., Mosley, C., Magklara, A., Lomvardas, S., Liberles, S.D., and Barnea, G. (2012). Neurons expressing trace amine-associated receptors project to discrete glomeruli and constitute an olfactory subsystem. Proc. Natl. Acad. Sci. U. S. A. 109, 13410-13415.

Johnstone, K.A., Ciborowski, K.L., Lubieniecki, K.P., Chow, W., Phillips, R.B., Koop, B.F., Jordan, W.C., and Davidson, W.S. (2009). Genomic organization and evolution of the vomeronasal type 2 receptorlike (OlfC) gene clusters in atlantic salmon, salmo salar. Mol. Biol. Evol. 26, 1117-1125.

Johnstone, K.A., Lubieniecki, K.P., Koop, B.F., and Davidson, W.S. (2012). Identification of olfactory receptor genes in Atlantic salmon Salmo salar. J. Fish Biol. 81, 559-575.

Jones, D.T., and Reed, R.R. (1989). Golf: An olfactory neuron specific-G protein involved in odorant signal transduction. Science. 244, 790-795.

Jones, W.D., Cayirlioglu, P., Grunwald Kadow, I., and Vosshall, L.B. (2007). Two chemosensory receptors together mediate carbon dioxide detection in Drosophila. Nature 445, 86-90.

Juilfs, D.M., Fülle, H.J., Zhao, A.Z., Houslay, M.D., Garbers, D.L., and Beavo, J.A. (1997). A subset of olfactory neurons that selectively express CGMP-stimulated phosphodiesterase (PDE2) and guanylyl 
cyclase-D define a unique olfactory signal transduction pathway. Proc. Natl. Acad. Sci. U. S. A. 94, 33883395.

Jungblut, L.D., Paz, D.A., López-Costa, J.J., and Pozzi, A.G. (2009). Heterogeneous distribution of G protein alpha subunits in the main olfactory and vomeronasal systems of Rhinella ( Bufo ) arenarum tadpoles. Zoolog. Sci. 26, 722-728.

Jungblut, L.D., Pozzi, A.G., and Paz, D.A. (2011). Larval development and metamorphosis of the olfactory and vomeronasal organs in the toad Rhinella (Bufo) arenarum (Hensel, 1867). Acta Zool. 92, 305-315.

Jungblut, L.D., Pozzi, A.G., and Paz, D.A. (2012). A putative functional vomeronasal system in anuran tadpoles. J. Anat. 221, 364-372.

Jungblut, L.D., Reiss, J.O., Paz, D.A., Andrea, I, Pozzi, G., and Jungblut, L.D. (2017). Quantitative comparative analysis of the nasal chemosensory organs of anurans during larval development and metamorphosis highlights the relative importance of chemosensory subsystems in the group. J. Morphol. 1-12.

Kapur, J.N., Sahoo, P.K., and Wong, A.K.C. (1985). A new method for gray-level picture thresholding using the entropy of the histogram. Comput. Vision, Graph. Image Process. 29, 273-285.

Karlson, P., and Lüscher, M. (1959). 'Pheromones': a New Term for a Class of Biologically Active Substances. Nature 183, 55-56.

Kassing, V., Engelmann, J., and Kurtz, R. (2013). Monitoring of single-cell responses in the optic tectum of adult zebrafish with dextran-coupled calcium dyes delivered via local electroporation. PLoS One 8, e62846.

Kauer, J.S. (2002). On the scents of smell in the salamander. Nature 417, 336-342.

Kavaliers, M., Choleris, E., and Pfaff, D.W. (2005). Recognition and avoidance of the odors of parasitized conspecifics and predators: Differential genomic correlates. Neurosci. Biobehav. Rev. 29, 1347-1359.

Kelley, D.B. (2004). Vocal communication in frogs. Curr. Opin. Neurobiol. 14, 751-757.

Kepchia, D., Sherman, B., Haddad, R., and Luetje, C.W. (2017). Mammalian odorant receptor tuning breadth persists across distinct odorant panels. PLoS One 12, 1-17.

Key, B., and Giorgi, P.P. (1986). Selective binding of soybean agglutinin to the olfactory system of Xenopus. Neuroscience 18, 507-515.

Kiemnec-Tyburczy, K.M., Woodley, S.K., Watts, R.A., Arnold, S.J., and Houck, L.D. (2012). Expression of vomeronasal receptors and related signaling molecules in the nasal cavity of a caudate amphibian (Plethodon shermani). Chem. Senses 37, 335-346.

Kikuyama, S., Toyoda, F., Ohmiya, Y., Matsuda, K., Tanaka, S., and Hayashi, H. (1995). Sodefrin: A female-attracting peptide pheromone in newt cloacal glands. Science. 267, 1643-1645.

Kimoto, H., Haga, S., Sato, K., and Touhara, K. (2005). Sex-specific peptides from exocrine glands stimulate mouse vomeronasal sensory neurons. Nature 437, 898-901.

Kishida, T., Thewissen, J.G.M., Hayakawa, T., Imai, H., and Agata, K. (2015). Aquatic adaptation and the evolution of smell and taste in whales. Zool. Lett. 1, 9.

Kishida, T., Go, Y., Tatsumoto, S., Tatsumi, K., Kuraku, S., and Toda, M. (2019). Loss of olfaction in sea 124 
snakes provides new perspectives on the aquatic adaptation of amniotes. Proc. R. Soc. B Biol. Sci. 286. Kleene, S.J., and Gesteland, R.C. (1991). Calcium-activated chloride conductance in frog olfactory cilia. J. Neurosci. 11, 3624-3629.

Kleerekoper, H., and Erkel, G.A. Van (1960). The olfactory apparatus of Petromyzon marinus. Can. J. Zool. 38, 209-223.

Klenoff, J.R., and Greer, C.A. (1998). Postnatal development of olfactory receptor cell axonal arbors. J. Comp. Neurol. 390, 256-267.

Kobayakawa, K., Kobayakawa, R., Matsumoto, H., Oka, Y., Imai, T., Ikawa, M., Okabe, M., Ikeda, T., Itohara, S., Kikusui, T., et al. (2007). Innate versus learned odour processing in the mouse olfactory bulb. Nature 450, 503-508.

Koide, T., Miyasaka, N., Morimoto, K., Asakawa, K., Urasaki, A., Kawakami, K., and Yoshihara, Y. (2009). Olfactory neural circuitry for attraction to amino acids revealed by transposon-mediated gene trap approach in zebrafish. Proc. Natl. Acad. Sci. U. S. A. 106, 9884-9889.

Kondo, R., Kaneko, S., Sun, H., Sakaizumi, M., and Chigusa, S.I. (2002). Diversification of olfactory receptor genes in the Japanese medaka fish, Oryzias latipes. Gene 282, 113-120.

Kondoh, D., Koshi, K., Ono, H.K., Sasaki, K., Nakamuta, N., and Taniguchi, K. (2013). Identification of G protein $\alpha$ subunits in the main olfactory system and vomeronasal system of the Japanese striped snake, Elaphe quadrivirgata. J. Vet. Med. Sci. 75, 381-385.

Kondoh, D., Kitayama, C., Yamaguchi, Y., Yanagawa, M., Kawai, Y.K., Suzuki, C., Itakura, R., Fujimoto, A., Satow, T., Kondo, S., et al. (2019). Nasal cavity of green sea turtles contains 3 independent sensory Epithelia. Chem. Senses 44, 427-434.

Korsching, S.I. (2008). The molecular evolution of teleost olfactory receptor gene families. In Chemosensory Systems in Mammals, Fishes, and Insects, S. Korsching, and W. Meyerhof, eds. (Berlin, Heidelberg: Springer), pp. 221-238.

Kozma, M.T., Schmidt, M., Ngo-Vu, H., Sparks, S.D., Senatore, A., and Derby, C.D. (2018). Chemoreceptor proteins in the Caribbean spiny lobster, Panulirus argus: Expression of ionotropic receptors, gustatory receptors, and TRP channels in two chemosensory organs and brain. PLoS One 13, e0203935.

Kramer, G. (1933). Untersuchungen über die Sinnesleistungen und das Orientierungsverhalten von Xenopus laevis, Daudin. Zool. Jahrbuch, Abteilung Allg. Zoolgoie Und Physiol. 52, 629-676.

Kratzing, J.E. (1975). The fine structure of the olfactory and vomeronasal organs of a lizard (Tiliqua scincoides scincoides). Cell Tissue Res. 159, 239-252.

Krautwurst, D., Yau, K.W., and Reed, R.R. (1998). Identification of ligands for olfactory receptors by functional expression of a receptor library. Cell 95, 917-926.

Kurahashi, T., and Yau, K.W. (1993). Co-existence of cationic and chloride components in odorantinduced current of vertebrate olfactory receptor cells. Nature $363,71-74$.

Laberge, F. (2008). Cytoarchitecture of the accessory olfactory bulb in the salamander Plethodon shermani. Brain Res. 1219, 32-45.

Laframboise, A.J., Ren, X., Chang, S., Dubuc, R., and Zielinski, B.S. (2007). Olfactory sensory neurons in 
the sea lamprey display polymorphisms. Neurosci. Lett. 414, 277-281.

Larriva-Sahd, J. (2008). The accessory olfactory bulb in the adult rat: A cytological study of its cell types, neuropil, neuronal modules, and interactions with the main olfactory system. J. Comp. Neurol. 510, 309-350.

Larsson, M.C., Domingos, A.I., Jones, W.D., Chiappe, M.E., Amrein, H., and Vosshall, L.B. (2004). Or83b encodes a broadly expressed odorant receptor essential for Drosophila olfaction. Neuron 43, 703-714.

Lee, S.G., Celestino, C.F., Stagg, J., Kleineidam, C., and Vickers, N.J. (2019). Moth pheromone-selective projection neurons with cell bodies in the antennal lobe lateral cluster exhibit diverse morphological and neurophysiological characteristics. J. Comp. Neurol. 527, 1443-1460.

Leinders-Zufall, T., Brennan, P., Widmayer, P., Chandramani S., P., Maul-Pavicic, A., Jäger, M., Li, X.H., Breer, H., Zufall, F., and Boehm, T. (2004). MHC class I peptides as chemosensory signals in the vomeronasal organ. Science. 306, 1033-1037.

Leinders-Zufall, T., Cockerham, R.E., Michalakis, S., Biel, M., Garbers, D.L., Reed, R.R., Zufall, F., and Munger, S.D. (2007). Contribution of the receptor guanylyl cyclase GC-D to chemosensory function in the olfactory epithelium. Proc. Natl. Acad. Sci. U. S. A. 104, 14507-14512.

Leinders-Zufall, T., Ishii, T., Chamero, P., Hendrix, P., Oboti, L., Schmid, A., Kircher, S., Pyrski, M., Akiyoshi, S., Khan, M., et al. (2014). A family of nonclassical class I MHC genes contributes to ultrasensitive chemodetection by mouse vomeronasal sensory neurons. J. Neurosci. 34, 5121-5133.

Lèvai, O., and Strotmann, J. (2003). Projection pattern of nerve fibers from the septal organ: Dil-tracing studies with transgenic OMP mice. Histochem. Cell Biol. 120, 483-492.

Leveteau (1992). The bilateral bulbar projections of the primary olfactory neurons in the frog J. 93104.

Lewcock, J.W., and Reed, R.R. (2004). A feedback mechanism regulates monoallelic odorant receptor expression. Proc. Natl. Acad. Sci. U. S. A. 101, 1069-1074.

Leypold, B.G., Yu, C.R., Leinders-Zufall, T., Kim, M.M., Zufall, F., and Axel, R. (2002). Altered sexual and social behaviors in trp2 mutant mice. Proc. Natl. Acad. Sci. U. S. A. 99, 6376-6381.

Li, Q. (2018). Deorphanization of olfactory trace amine-associated receptors. Methods Mol. Biol. 1820, 21-31.

Li, W., Sorensen, P.W., and Gallaher, D.D. (1995). The olfactory system of migratory adult sea lamprey (Petromyzon marinus) is specifically and acutely sensitive to unique bile acids released by conspecific larvae. J. Gen. Physiol. 105, 569-587.

Libants, S., Carr, K., Wu, H., Teeter, J.H., Chung-Davidson, Y.W., Zhang, Z., Wilkerson, C., and Li, W. (2009). The sea lamprey Petromyzon marinus genome reveals the early origin of several chemosensory receptor families in the vertebrate lineage. BMC Evol. Biol. 9, 180.

Liberles, S.D., and Buck, L.B. (2006). A second class of chemosensory receptors in the olfactory epithelium. 442, 645-650.

Liberles, S.D., Horowitz, L.F., Kuang, D., Contos, J.J., Wilson, K.L., Siltberg-Liberles, J., Liberles, D.A., and Buck, L.B. (2009). Formyl peptide receptors are candidate chemosensory receptors in the vomeronasal organ. Proc. Natl. Acad. Sci. U. S. A. 106, 9842-9849. 
Liman, E.R., Corey, D.P., and Dulac, C. (1999). TRP2: A candidate transduction channel for mammalian pheromone sensory signaling. Proc. Natl. Acad. Sci. U. S. A. 96, 5791-5796.

Lin, W., Margolskee, R., Donnert, G., Hell, S.W., and Restrepo, D. (2007). Olfactory neurons expressing transient receptor potential channel M5 (TRPM5) are involved in sensing semiochemicals. Proc. Natl. Acad. Sci. U. S. A. 104, 2471-2476.

Lin, W., Ezekwe, E.A.D., Zhao, Z., Liman, E.R., and Restrepo, D. (2008). TRPM5-expressing microvillous cells in the main olfactory epithelium. BMC Neurosci. 9, 1-13.

Lindemann, L., and Hoener, M.C. (2005). A renaissance in trace amines inspired by a novel GPCR family. Trends Pharmacol. Sci. 26, 274-281.

Lindemann, L., Ebeling, M., Kratochwil, N.A., Bunzow, J.R., Grandy, D.K., and Hoener, M.C. (2005). Trace amine-associated receptors form structurally and functionally distinct subfamilies of novel G proteincoupled receptors. Genomics 85, 372-385.

Little, C. (1990). The terrestrial invasion: an ecophysiological approach to the origins of land animals (Cambridge, UK: Cambridge University Press).

Llahi, S., and García-Verdugo, J.M. (1989). Neuronal organization of the accessory olfactory bulb of the lizard Podarcis hispanica: Golgi study. J. Morphol. 202, 13-28.

Lomvardas, S., Barnea, G., Pisapia, D.J., Mendelsohn, M., Kirkland, J., and Axel, R. (2006). Interchromosomal Interactions and Olfactory Receptor Choice. Cell 126, 403-413.

Lu, T., Qiu, Y.T., Wang, G., Kwon, J.Y., Rutzler, M., Kwon, H.W., Pitts, R.J., van Loon, J.J.A., Takken, W., Carlson, J.R., et al. (2007). Odor coding in the maxillary palp of the malaria vector mosquito Anopheles gambiae. Curr. Biol. 17, 1533-1544.

Lucas, P., Ukhanov, K., Leinders-Zufall, T., and Zufall, F. (2003). A diacylglycerol-gated cation channel in vomeronasal neuron dendrites is impaired in TRPC2 mutant mice: Mechanism of pheromone transduction. Neuron 40, 551-561.

Luo, Y., Lu, S., Chen, P., Wang, D., and Halpern, M. (1994). Identification of chemoattractant receptors and G-proteins in the vomeronasal system of garter snakes. J. Biol. Chem. 269, 16867-16877.

Luu, P., Acher, F., Bertrand, H.O., Fan, J., and Ngai, J. (2004). Molecular determinants of ligand selectivity in a vertebrate odorant receptor. J. Neurosci. 24, 10128-10137.

Mackay-Sim, A., Duvall, D., and Graves, B.M. (1985). The west indian manatee (Trichechus manatus) lacks a vomeronasal organ. Brain. Behav. Evol. 27, 186-194.

Macrides, F., and Schneider, S.P. (1982). Laminar organization of mitral and tufted cells in the main olfactory bulb of the adult hamster. J. Comp. Neurol. 208, 419-430.

Macrides, F., Schoenfeld, T.A., Marchand, J.E., and Clancy, A.N. (1985). Evidence for morphologically, neurochemically and functionally heterogeneous classes of mitral and tufted cells in the olfactory bulb. Chem. Senses 10, 175-202.

Mair, R.G., Gesteland, R.C., and Blank, D.L. (1982). Changes in morphology and physiology of olfactory receptor cilia during development. Neuroscience 7, 3091-3103.

Malnic, B. (2007). Searching for the ligands of odorant receptors. Mol. Neurobiol. 35, 175-181.

Malnic, B., Hirono, J., Sato, T., and Buck, L.B. (1999). Combinatorial receptor codes for odors. Cell 96, 
713-723.

Manzini, I., and Korsching, S. (2011). The peripheral olfactory system of vertebrates: molecular, structural and functional basics of the sense of smell. E-Neuroforum 17, 68-77.

Manzini, I., and Schild, D. (2003). cAMP-independent olfactory transduction of amino acids in Xenopus laevis tadpoles. J. Physiol. 551, 115-123.

Manzini, I., and Schild, D. (2004). Classes and narrowing selectivity of olfactory receptor neurons of Xenopus laevis tadpoles. J. Gen. Physiol. 123, 99-107.

Manzini, I., and Schild, D. (2010). Olfactory coding in larvae of the African Clawed frog Xenopus laevis. In The Neurobiology of Olfaction, A. Menini, ed. (Boca Raton, FL: CRC Press/Taylor \& Francis), pp. 114126.

Manzini, I., Rössler, W., and Schild, D. (2002). cAMP-independent responses of olfactory neurons in Xenopus laevis tadpoles and their projection onto olfactory bulb neurons. J. Physiol. 545, 475-484.

Manzini, I., Heermann, S., Czesnik, D., Brase, C., Schild, D., and Rössler, W. (2007). Presynaptic protein distribution and odour mapping in glomeruli of the olfactory bulb of Xenopus laevis tadpoles. Eur. J. Neurosci. 26, 925-934.

Marchand, J.E., Yang, X., Chikaraishi, D., Krieger, J., Breer, H., Kauer, J.S., Journal, T., Neurology, C., Marchand, J.E., Yang, X., et al. (2004). Olfactory receptor gene expression in tiger salamander olfactory epithelium. J. Comp. Neurol. 474, 453-467.

Marcucci, F., Maier-Balough, E., Zou, D.-J., and Firestein, S. (2011). Exuberant growth and synapse formation of olfactory sensory neuron axonal arborizations. J. Comp. Neurol. 519, 3713-3726.

Maresh, A., Rodriguez Gil, D., Whitman, M.C., and Greer, C.A. (2008). Principles of glomerular organization in the human olfactory bulb - Implications for odor processing. PLoS One 3, e2640.

Markenscoff-Papadimitriou, E., Allen, W.E., Colquitt, B.M., Goh, T., Murphy, K.K., Monahan, K., Mosley, C.P., Ahituv, N., and Lomvardas, S. (2014). Enhancer interaction networks as a means for singular olfactory receptor expression. Cell 159, 543-557.

Marra, N.J., Stanhope, M.J., Jue, N.K., Wang, M., Sun, Q., Bitar, P.P., Richards, V.P., Komissarov, A., Rayko, M., Kliver, S., et al. (2019). White shark genome reveals ancient elasmobranch adaptations associated with wound healing and the maintenance of genome stability. Proc. Natl. Acad. Sci. U. S. A. $116,4446-4455$.

Martini, S., Silvotti, L., Shirazi, A., Ryba, N.J.P., and Tirindelli, R. (2001). Co-expression of putative pheromone receptors in the sensory neurons of the vomeronasal organ. J. Neurosci. 21, 843-848.

Masche, S., Zimmermann, H., and Pröhl, H. (2010). Description and ecological observations of the tadpole of Ranitomeya variabilis (Anura: Dendrobatidae). South Am. J. Herpetol. 5, 207-211.

Matsunami, H., and Buck, L.B. (1997). A multigene family encoding a diverse array of putative pheromone receptors in mammals. Cell 90, 775-784.

Mayer, M.L. (2005). Glutamate receptor ion channels. Curr. Opin. Neurobiol. 15, 282-288.

McCarroll, S.A., Li, H., and Bargmann, C.I. (2005). Identification of transcriptional regulatory elements in chemosensory receptor genes by probabilistic segmentation. Curr. Biol. 15, 347-352.

McKenzie, S.K., and Kronauer, D.J.C. (2018). The genomic architecture and molecular evolution of ant 128 
odorant receptors. Genome Res. 28, 1757-1765.

Measey, J. (2016). Overland movement in African clawed frogs (Xenopus laevis ): a systematic review. PeerJ 4, e2474.

Meisami, E., and Bhatnagar, K.P. (1998). Structure and diversity in mammalian accessory olfactory bulb. Microsc. Res. Tech. 43, 476-499.

Menco, B.P.M. (1980). Qualitative and quantitative freeze-fracture studies on olfactory and nasal respiratory structures of frog, ox, rat, and dog - I. A general survey. Cell Tissue Res. 207, 183-209.

Meredith, T.L., Kajiura, S.M., and Hansen, A. (2013). The somatotopic organization of the olfactory bulb in elasmobranchs. J. Morphol. 274, 447-455.

Meyer, D.L., Jadhao, A.G., Bhargava, S., and Kicliter, E. (1996). Bulbar representation of the "waternose" during Xenopus ontogeny. Neurosci. Lett. 220, 109-112.

Mezler, M., Konzelmann, S., Freitag, J., Rössler, P., and Breer, H. (1999). Expression of olfactory receptors during development in Xenopus laevis. J. Exp. Biol. 202, 365-376.

Mezler, M., Fleischer, J., and Breer, H. (2001). Characteristic features and ligand specificity of two olfactory receptor classes from Xenopus laevis. 2997, 2987-2997.

Migeotte, I., Communi, D., and Parmentier, M. (2006). Formyl peptide receptors: A promiscuous subfamily of $\mathrm{G}$ protein-coupled receptors controlling immune responses. Cytokine Growth Factor Rev. $17,501-519$.

Miyamichi, K., Serizawa, S., Kimura, H.M., and Sakano, H. (2005). Continuous and overlapping expression domains of odorant receptor genes in the olfactory epithelium determine the dorsal/ventral positioning of glomeruli in the olfactory bulb. J. Neurosci. 25, 3586-3592.

Miyasaka, N., Arimatsu, Y., and Takiguchihayashi, K. (1999). Foreign gene expression in an organotypic culture of cortical anlage after in vivo electroporation. Neuroreport 10, 2319-2323.

Mohrhardt, J., Nagel, M., Fleck, D., Ben-Shaul, Y., and Spehr, M. (2018). Signal detection and coding in the accessory olfactory system. Chem. Senses 43, 667-695.

Mombaerts, P. (1999). Seven-transmembrane proteins as odorant and chemosensory receptors. Science 286, 707-711.

Mombaerts, P. (2006). Axonal wiring in the mouse olfactory system. Annu. Rev. Cell Dev. Biol. 22, 713737.

Mombaerts, P., Wang, F., Dulac, C., Chao, S.K., Nemes, A., Mendelsohn, M., Edmondson, J., and Axel, R. (1996). Visualizing an olfactory sensory map. Cell 87, 675-686.

Monahan, K., and Lomvardas, S. (2015). Monoallelic expression of olfactory receptors. Annu. Rev. Cell Dev. Biol. 31, 721-740.

Monahan, K., Schieren, I., Cheung, J., Mumbey-Wafula, A., Monuki, E.S., and Lomvardas, S. (2017). Cooperative interactions enable singular olfactory receptor expression in mouse olfactory neurons. Elife 6, e28620.

Mori, K., Kishi, K., and Ojima, H. (1983). Distribution of dendrites of mitral, displaced mitral, tufted, and granule cells in the rabbit olfactory bulb. J. Comp. Neurol. 219, 339-355. 
Mori, K., von Campenhause, H., and Yoshihara, Y. (2000). Zonal organization of the mammalian main and accessory olfactory systems. Philos. Trans. R. Soc. Lond. B. Biol. Sci. 355, 1801-1812.

Mori, K., Takahashi, Y.K., Igarashi, K.M., and Yamaguchi, M. (2006). Maps of odorant molecular features in the mammalian olfactory bulb. Physiol. Rev. 86, 409-433.

Munger, S.D., Leinders-Zufall, T., and Zufall, F. (2009). Subsystem organization of the mammalian sense of smell. Annu. Rev. Physiol. 71, 115-140.

Munger, S.D., Leinders-Zufall, T., McDougall, L.M., Cockerham, R.E., Schmid, A., Wandernoth, P., Wennemuth, G., Biel, M., Zufall, F., and Kelliher, K.R. (2010). An olfactory subsystem that detects carbon disulfide and mediates food-related social learning. Curr. Biol. 20, 1438-1444.

Murphy, C.A., Stacey, N.E., and Corkum, L.D. (2001). putative steroidal pheromones in the round goby, Neogobius melanostomus: olfactory and behaviroal responses. J. Chem. Ecol. 27, 443-470.

Nagayama, S. (2010). Differential axonal projection of mitral and tufted cells in the mouse main olfactory system. Front. Neural Circuits 4, 120.

Nagayama, S., Homma, R., and Imamura, F. (2014). Neuronal organization of olfactory bulb circuits. Front. Neural Circuits 8, 98.

Naito, T., Saito, Y., Yamamoto, J., Nozaki, Y., Tomura, K., Hazama, M., Nakanishi, S., and Brenner, S. (1998). Putative pheromone receptors related to the Ca2+-sensing receptor in Fugu. Proc. Natl. Acad. Sci. U. S. A. 95, 5178-5181.

Nakada, T., Hagino-Yamagishi, K., Nakanishi, K., Yokosuka, M., Saito, T.R., Toyoda, F., Hasunuma, I., Nakakura, T., and Kikuyama, S. (2014). Expression of G proteins in the olfactory receptor neurons of the newt Cynops pyrrhogaster: Their unique projection into the olfactory bulbs. J. Comp. Neurol. 522, 3501-3519.

Nakamura, T., and Gold, G.H. (1987). A cyclic nucleotide-gated conductance in olfactory receptor cilia. Nature 325, 442-444.

Nakamuta, N., Nakamuta, S., Kato, H., and Yamamoto, Y. (2016a). Morphological study on the olfactory systems of the snapping turtle, Chelydra serpentina. Tissue Cell 48, 145-151.

Nakamuta, S., Nakamuta, N., Taniguchi, K., and Taniguchi, K. (2012). Histological and ultrastructural characteristics of the primordial vomeronasal organ in lungfish. Anat. Rec. 295, 481-491.

Nakamuta, S., Yokosuka, M., Taniguchi, K., Yamamoto, Y., and Nakamuta, N. (2016b). Immunohistochemical analysis for $\mathrm{G}$ protein in the olfactory organs of soft-shelled turtle, Pelodiscus sinensis. J. Vet. Med. Sci. 78, 245-250.

Nara, K., Saraiva, L.R., Ye, X., and Buck, L.B. (2011). A large-scale analysis of odor coding in the olfactory epithelium. J. Neurosci. 31, 9179-9191.

Nef, S., Allaman, I., Fiumelli, H., De Castro, E., and Nef, P. (1996). Olfaction in birds: Differential embryonic expression of nine putative odorant receptor genes in the avian olfactory system. Mech. Dev. 55, 65-77.

Nei, M., and Rooney, A.P. (2005). Concerted and birth-and-death evolution of multigene families. Annu. Rev. Genet. 39, 121-152.

Nei, M., Niimura, Y., and Nozawa, M. (2008). The evolution of animal chemosensory receptor gene 
repertoires: Roles of chance and necessity. Nat. Rev. Genet. 9, 951-963.

Neumann, E., Kakorin, S., and Tœnsing, K. (1999). Fundamentals of electroporative delivery of drugs and genes. Bioelectrochemistry Bioenerg. 48, 3-16.

Nezlin, L.P., and Schild, D. (2000). Structure of the olfactory bulb in tadpoles of Xenopus laevis. Cell Tissue Res. 302, 21-29.

Nezlin, L.P., and Schild, D. (2005). Individual olfactory sensory neurons project into more than one glomerulus in Xenopus laevis tadpole olfactory bulb. J. Comp. Neurol. 481, 233-239.

Nezlin, L.P., Heermann, S., Schild, D., and Rössler, W. (2003). Organization of glomeruli in the main olfactory bulb of Xenopus laevis tadpoles. J. Comp. Neurol. 464, 257-268.

Ngai, J., Dowling, M.M., Buck, L., Axel, R., and Chess, A. (2003). The family of genes encoding odorant receptors in the channel catfish. Cell 72, 657-666.

Nieuwenhuys, R. (1966). Comparative anatomy of olfactory centres and tracts. Prog. Brain Res. 23, 164.

Nieuwkoop, D.P., and Faber, J. (1994). Normal table of Xenopus laevis (Daudin). Garland, New York.

Niimura, Y. (2009). Evolutionary dynamics of olfactory receptor genes in chordates: interaction between environments and genomic contents. Hum. Genomics 4, 107-118.

Niimura, Y., and Nei, M. (2006). Evolutionary dynamics of olfactory and other chemosensory receptor genes in vertebrates. J. Hum. Genet. 51, 505-517.

Niimura, Y., and Nei, M. (2007). Extensive gains and losses of olfactory receptor genes in mammalian evolution. PLoS One 2, e708.

Nikaido, M. (2019). Evolution of V1R pheromone receptor genes in vertebrates: diversity and commonality. Genes Genet Syst. 94, 141-149.

Nikaido, M., Noguchi, H., Nishihara, H., Toyoda, A., Suzuki, Y., Kajitani, R., Suzuki, H., Okuno, M., Aibara, M., Ngatunga, B.P., et al. (2013a). Coelacanth genomes reveal signatures for evolutionary transition from water to land. Genome Res. 23, 1740-1748.

Nikaido, M., Suzuki, H., Toyoda, A., Fujiyama, A., Hagino-Yamagishi, K., Kocher, T.D., Carleton, K., and Okada, N. (2013b). Lineage-specific expansion of vomeronasal type 2 receptor-like (OlfC) genes in cichlids may contribute to diversification of amino acid detection systems. Genome Biol. Evol. 5, 711722.

Norlin, E.M., Gussing, F., and Berghard, A. (2003). Vomeronasal phenotype and behavioral alterations in Gai2 mutant mice. Curr. Biol. 13, 1214-121.

Nowack, C., and Vences, M. (2016). Ontogenetic development of the derived olfactory system of the mantellid frog Mantidactylus betsileanus. Anat. Rec. 299, 943-950.

Nowack, C., Jordan, S., and Wittmer, C. (2013). The recessus olfactorius: A cryptic olfactory organ of anuran amphibians. In Chemical Signals in Vertebrates, M.L. East, and M. Dehnhard, eds. (New York: Springer Science+Business Media), pp. 37-48.

Offner, T. (2020). Wiring and information processing in the olfactory bulb of larval Xenopus laevis. Universits of Göttingen. 
Oka, Y. (1983). Golgi, electron-microscopic and combined golgi-electron-microscopic studies of the mitral cells in the goldfish olfactory bulb. Neuroscience $8,723-742$.

Oka, Y., Saraiva, L.R., and Korsching, S.I. (2012). Crypt neurons express a single v1r-related ora gene. Chem. Senses 37, 219-227.

Olivares, J., and Schmachtenberg, O. (2019). An update on anatomy and function of the teleost olfactory system. PeerJ 7, e7808.

Orona, E., Rainer, E.C., and Scott, J.W. (1984). Dendritic and axonal organization of mitral and tufted cells in the rat olfactory bulb. J. Comp. Neurol. 226, 346-356.

Orton, G.L. (1953). The systematics of vertebrate larvae. Syst. Zool. 2, 63-75.

Ota, T., Nikaido, M., Suzuki, H., Hagino-Yamagishi, K., and Okada, N. (2012). Characterization of V1R receptor (ora) genes in Lake Victoria cichlids. Gene 499, 273-279.

Ottoson, D. (1959). Comparison of slow potentials evoked in the frog's nasal mucosa and Olfactory bulb by natural stimulation. Acta Physiol. Scand. 149-159.

Pace, U., Hanski, E., Salomon, Y., and Lancet, D. (1985). Odorant-sensitive adenylate cyclase may mediate olfactory reception. Nature 316, 255-258.

Pacifico, R., Dewan, A., Cawley, D., Guo, C., and Bozza, T. (2012). An olfactory subsystem that mediates high-sensitivity detection of volatile amines. Cell Rep. 2, 76-88.

Park, D., and Propper, C.R. (2002). The olfactory organ is activated by a repelling pheromone in the red-spotted newt Notophthalmus viridescens . Korean J. Biol. Sci. 6, 233-237.

Park, D., McGuire, J.M., Majchrzak, A.L., Ziobro, J.M., and Eisthen, H.L. (2004). Discrimination of conspecific sex and reproductive condition using chemical cues in axolotls (Ambystoma mexicanum). J. Comp. Physiol. A Neuroethol. Sensory, Neural, Behav. Physiol. 190, 415-427.

Paterson, N.F., and Hindle, E. (1951). The nasal cavities of the Toad Hemipipa carvalhoi and other Pipidae. Proc. Zool. Soc. London 121, 381-415.

Pedregosa, F., Varoquaux, G., Gramfort, A., Michel, V., Thirion, B., Grisel, O., Blondel, M., Prettenhofer, P., Weiss, R., Dubourg, V., et al. (2011). Scikit-learn: Machine Learning in Python. J. Mach. Learn. Res. $12,2825-2830$.

Peng, H., Ruan, Z., Long, F., Simpson, J.H., and Myers, E.W. (2010). V3D enables real-time 3D visualization and quantitative analysis of large-scale biological image data sets. Nat. Biotechnol. 28, 348-353.

Pfister, P., and Rodriguez, I. (2005). Olfactory expression of a single and highly variable V1r pheromone receptor-like gene in fish species. 102, 5489-5494.

Pinching, a J., and Powell, T.P. (1971). The neuropil of the periglomerular region of the olfactory bulb. J. Cell Sci. 9, 379-409.

Du Plessis, S.S. (1966). Stimulation of spawning in Xenopus laevis by fowl manure. Nature 211, 10921092.

Pnevmatikakis, E.A., and Giovannucci, A. (2017). NoRMCorre: An online algorithm for piecewise rigid motion correction of calcium imaging data. J. Neurosci. Methods 291, 83-94. 
Pnevmatikakis, E.A., Gao, Y., Soudry, D., Pfau, D., Lacefield, C., Poskanzer, K., Bruno, R., Yuste, R., and Paninski, L. (2014). A structured matrix factorization framework for large scale calcium imaging data analysis. ArXiv Prepr. ArXiv1409.2903.

Pnevmatikakis, E.A., Soudry, D., Gao, Y., Machado, T.A., Merel, J., Pfau, D., Reardon, T., Mu, Y., Lacefield, C., Yang, W., et al. (2016). Simultaneous Denoising, Deconvolution, and Demixing of Calcium Imaging Data. Neuron 89, 285-299.

Polese, G., Bertapelle, C., and Di Cosmo, A. (2016). Olfactory organ of Octopus vulgaris: Morphology, plasticity, turnover and sensory characterization. Biol. Open 5, 611-619.

Preibisch, S., Saalfeld, S., and Tomancak, P. (2009). Globally optimal stitching of tiled 3D microscopic image acquisitions. Bioinformatics 25, 1463-1465.

Del Punta, K., Leinders-Zufall, T., Rodriguez, I., Jukam, D., Wysocki, C.J., Ogawa, S., Zufall, F., and Mombaerts, P. (2002a). Deficient pheromone responses in mice lacking a cluster of vomeronasal receptor genes. Nature $419,70-74$.

Del Punta, K., Puche, A., Adams, N.C., Rodriguez, I., and Mombaerts, P. (2002b). A divergent pattern of sensory axonal projections is rendered convergent by second-order neurons in the accessory olfactory bulb. Neuron 35, 1057-1066.

Quinzio, S.I., and Reiss, J.O. (2018). The ontogeny of the olfactory system in ceratophryid frogs (Anura, Ceratophryidae). J. Morphol. 279, 37-49.

Raible, F., Tessmar-Raible, K., Arboleda, E., Kaller, T., Bork, P., Arendt, D., and Arnone, M.I. (2006). Opsins and clusters of sensory G-protein-coupled receptors in the sea urchin genome. Dev. Biol. 300, 461-475.

Ramón y Cajal, S. (1911). Histologie du systeme nerveux de l'homme et des vertebres (Paris: Maloine).

Reese, T.S. (1965). Olfactory cilia in the frog. J. Cell Biol. 25, 209-230.

Reiss, J.O., and Burd, G.D. (1997a). Metamorphic remodeling of the primary olfactory projection in Xenopus: Developmental independence of projections from olfactory neuron subclasses. J. Neurobiol. $32,213-222$.

Reiss, J.O., and Burd, G.D. (1997b). Cellular and molecular interactions in the development of the Xenopus olfactory system. Semin. Cell Dev. Biol. 8, 171-179.

Reiss, J.O., and Eisthen, H.L. (2008). Comparative anatomy and physiology of chemical senses in amphibians. In Sensory Evolution on the Threshold: Adaptations in Secondarily Aquatic Vertebrates, J. Thewissen, and S. Nummela, eds. (Oakland, CA: University of California Press), pp. 43-63.

Ren, X., Chang, S., Laframboise, A., Green, W., Dubuc, R., and Zielinski, B. (2009). Projections from the accessory olfactory organ into the medial region of the olfactory bulb in the sea lamprey (Petromyzon marinus): A novel vertebrate sensory structure? J. Comp. Neurol. 516, 105-116.

Ressler, K.J., Sullivan, S.L., and Buck, L.B. (1994). Information coding in the olfactory system: Evidence for a stereotyped and highly organized epitope map in the olfactory bulb. Cell 79, 1245-1255.

Riabinina, O., Task, D., Marr, E., Lin, C.C., Alford, R., O'Brochta, D.A., and Potter, C.J. (2016). Organization of olfactory centres in the malaria mosquito Anopheles gambiae. Nat. Commun. 7, 13010.

Rivière, S., Challet, L., Fluegge, D., Spehr, M., and Rodriguez, I. (2009). Formyl peptide receptor-like 
proteins are a novel family of vomeronasal chemosensors. Nature 459, 574-577.

Roberts, R.E., Motti, C.A., Baughman, K.W., Satoh, N., Hall, M.R., and Cummins, S.F. (2017). Identification of putative olfactory G-protein coupled receptors in Crown-of-Thorns starfish, Acanthaster planci. BMC Genomics 18, 400.

Robertson, H.M. (2015). The insect chemoreceptor superfamily is ancient in animals. Chem. Senses 40, 609-614.

Robertson, H.M. (2019). Molecular evolution of the major arthropod chemoreceptor gene families. Annu. Rev. Entomol. 64, 227-242.

Robertson, H.M., Warr, C.G., and Carlson, J.R. (2003). Molecular evolution of the insect chemoreceptor gene superfamily in Drosophila melanogaster. Proc. Natl. Acad. Sci. U. S. A. 100, 14537-14542.

Rodolfo-Masera (1943). Su l'esistenza di un particolare organo olfattivo nel setto nasale della cavia e di altri roditori. Arch. Ital. Anat. Embryol. 48, 157-212.

Rodriguez, I., Feinstein, P., and Mombaerts, P. (1999). Variable patterns of axonal projections of sensory neurons in the mouse vomeronasal system. Cell 97, 199-208.

Rodriguez, I., Del Punta, K., Rothman, A., Ishii, T., and Mombaerts, P. (2002). Multiple new and isolated families within the mouse superfamily of V1r vomeronasal receptors. Nat. Neurosci. 5, 134-140.

Roelants, K., Haas, A., and Bossuyt, F. (2011). Anuran radiations and the evolution of tadpole morphospace. Proc. Natl. Acad. Sci. U. S. A. 108, 8731-8736.

Roland, A.B., O'Connell, L.A., and O'Connell, L.A. (2015). Poison frogs as a model system for studying the neurobiology of parental care. Curr. Opin. Behav. Sci. 6, 76-81.

Rollmann, S.M., Houck, L.D., and Feldhoff, R.C. (1999). Proteinaceous pheromone affecting female receptivity in a terrestrial salamander. Science. 285, 1907-1909.

Roppolo, D., Ribaud, V., Jungo, V.P., Lüscher, C., and Rodriguez, I. (2006). Projection of the Grüneberg ganglion to the mouse olfactory bulb. Eur. J. Neurosci. 23, 2887-2894.

Różański, J.J., and Żuwała, K.D. (2019). The influence of habitat on olfactory organ structure in selected species of salamanders (Salamandridae, Caudata). Zool. Anz. 281, 1-10.

Rünnenburger, K., Breer, H., and Boekhoff, I. (2002). Selective G protein $\beta \gamma$-subunit compositions mediate phospholipase $\mathrm{C}$ activation in the vomeronasal organ. Eur. J. Cell Biol. 81, 539-547.

Ryba, N.J.P., Tirindelli, R., Umana, F., and Parma, U. (1997). A new multigene family of putative pheromone receptors. $19,371-379$.

Saito, H., Chi, Q., Zhuang, H., Matsunami, H., and Mainland, J.D. (2009). Odor coding by a mammalian receptor repertoire. Sci. Signal. 2, 1-15.

Salazar, I., and Sánchez Quinteiro, P. (2009). The risk of extrapolation in neuroanatomy: The case of the mammalian vomeronasal system. Front. Neuroanat. 3, 1-12.

Sansone, A., Syed, A.S., Tantalaki, E., Korsching, S.I., and Manzini, I. (2014). Trpc2 is expressed in two olfactory subsystems, the main and the vomeronasal system of larval Xenopus laevis. J. Exp. Biol. 217.

Sansone, A., Hassenklöver, T., Offner, T., Fu, X., Holy, T.E., and Manzini, I. (2015). Dual processing of sulfated steroids in the olfactory system of an anuran amphibian. Front. Cell. Neurosci. 9, 373. 
Saraiva, L.R., and Korsching, S.I. (2007). A novel olfavtory receptor gene family in teleost fish. Genome Res. 1448-1457.

Sato, Y. (2005). Mutually exclusive glomerular innervation by two distinct types of olfactory sensory neurons revealed in transgenic zebrafish. J. Neurosci. 25, 4889-4897.

Sato, K., and Sorensen, P.W. (2018). The chemical sensitivity and electrical activity of individual olfactory sensory neurons to a range of sex pheromones and food odors in the goldfish. Chem. Senses 43, 249-260.

Sato, K., Pellegrino, M., Nakagawa, T., Nakagawa, T., Vosshall, L.B., and Touhara, K. (2008). Insect olfactory receptors are heteromeric ligand-gated ion channels. Nature 452, 1002-1006.

Satou, M. (1990). Synaptic organization, local neuronal circuitry, and functional segregation of the teleost olfactory bulb. Prog. Neurobiol. 34, 115-142.

Savage, R.M. (1965). External stimulus for the natural spawning of Xenopus laevis [42]. Nature 205, 618-619.

Scalia, F., Gallousis, G., and Roca, S. (1991a). A Note on the organization of the amphibian olfactory bulb. J. Comp. Neurol. 305, 435-442.

Scalia, F., Gallousis, G., and Roca, S. (1991b). Differential projections of the main and accessory olfactory bulb in the frog. J. Comp. Neurol. 305, 443-461.

Schild, D., and Restrepo, D. (1998). Transduction mechanisms in vertebrate olfactory receptor cells. Physiol. Rev. 78, 429-466.

Schindelin, J., Arganda-Carreras, I., Frise, E., Kaynig, V., Longair, M., Pietzsch, T., Preibisch, S., Rueden, C., Saalfeld, S., Schmid, B., et al. (2012). Fiji: an open-source platform for biological-image analysis. Nat. Methods 9, 676-682.

Schmachtenberg, O. (2006). Histological and electrophysiological properties of crypt cells from the olfactory epithelium of the marine teleost Trachurus symmetricus. J. Comp. Neurol. 495, 113-121.

Schmidt, A., and Wake, M.H. (1990). Olfactory and vomeronasal systems of caecilians (Amphibia: Gymnophiona). J. Morphol. 205, 255-268.

Schmidt, M., Van Ekeris, L., and Ache, B.W. (1992). Antennular projections to the midbrain of the spiny lobster. I. Sensory innervation of the lateral and medial antennular neuropils. J. Comp. Neurol. 318, 277-290.

Schneider, D. (1964). Insect Antennae. Annu. Rev. Entomol. 9, 103-122.

Schwob, J.E. (2002). Neural regeneration and the peripheral olfactory system. Anat. Rec. 269, 33-49.

Schwob, J.E., Jang, W., Holbrook, E.H., Lin, B., Herrick, D.B., Peterson, J.N., and Hewitt Coleman, J. (2017). Stem and progenitor cells of the mammalian olfactory epithelium: Taking poietic license. J. Comp. Neurol. 525, 1034-1054.

Scott, W.B. (1887). Notes on the development of petromyzon. J Morphol 1, 253-310.

Scott, A.M., Zhang, Z., Jia, L., Li, K., Zhang, Q., Dexheimer, T., Ellsworth, E., Ren, J., Chung-Davidson, Y.W., Zu, Y., et al. (2019). Spermine in semen of male sea lamprey acts as a sex pheromone. PLoS Biol. $17,1-24$. 
Seale, D.B. (1982). Obligate and facultative suspension feeding in anuran larvae: feeding regulation in Xenopus and Rana. Biol. Bull. 162, 214-231.

Sengupta, P., Chou, J.H., and Bargmann, C.I. (1996). odr-10 Encodes a seven transmembrane domain olfactory receptor required for responses to the odorant diacetyl. Cell 84, 899-909.

Serizawa, S., Ishii, T., Nakatani, H., Tsuboi, A., Nagawa, F., Asano, M., Sudo, K., Sakagami, J., Sakano, H., Ijiri, T., et al. (2000). Mutually exclusive expression of odorant receptor transgenes. Nat. Neurosci. 3, 687-693.

Serizawa, S., Miyamichi, K., Nakatani, H., Suzuki, M., Saito, M., Yoshihara, Y., and Sakano, H. (2003). Negative Feedback Regulation Ensures the One Receptor-One Olfactory Neuron Rule in Mouse. Science. 302, 2088-2094.

Serizawa, S., Miyamichi, K., Takeuchi, H., Yamagishi, Y., Suzuki, M., and Sakano, H. (2006). A neuronal identity code for the odorant receptor-specific and activity-dependent axon sorting. Cell 127, 10571069.

Sharma, K., Syed, A.S., Ferrando, S., Mazan, S., and Korsching, S.I. (2019). The chemosensory receptor repertoire of a true shark is dominated by a single olfactory receptor family. Genome Biol. Evol. 11, 398-405.

Shi, P., and Zhang, J. (2007). Comparative genomic analysis identifies an evolutionary shift of vomeronasal receptor gene repertoires in the vertebrate transition from water to land. Genome Res. $17,166-174$.

Shinn, E.A., and Dole, J.W. (1978). Evidence for a role for olfactory cues in the feeding response of leopard frogs, Rana pipiens. Herpetologica 34, 167-172.

Shinn, E.A., and Dole, J.W. (1979). Lipid components of prey odors elicit feeding responses in western toads (Bufo boreas). Copeia 2, 275-278.

Shiraiwa, T. (2008). Multimodal chemosensory integration through the maxillary palp in drosophila. PLoS One 3, e2191.

Shykind, B.M., Christy Rohani, S., Nemes, A., Mendelsohn, M., Sun, Y., Axel, R., and Barnea, G. (2004). Gene switching and the stability of odorant receptor gene choice. Cell 117, 801-815.

Silva, L., and Antunes, A. (2017). Vomeronasal receptors in vertebrates and the evolution of pheromone detection. Annu. Rev. Anim. Biosci. 5, 353-370.

Silvotti, L., Moiani, A., Gatti, R., and Tirindelli, R. (2007). Combinatorial co-expression of pheromone receptors, V2Rs. J. Neurochem. Neurochem. 103, 1753-1763.

Silvotti, L., Cavalca, E., Gatti, R., Percudani, R., and Tirindelli, R. (2011). A recent class of chemosensory neurons developed in mouse and rat. PLoS One 6, e24462.

Smart, R., Kiely, A., Beale, M., Vargas, E., Carraher, C., Kralicek, A. V., Christie, D.L., Chen, C., Newcomb, R.D., and Warr, C.G. (2008). Drosophila odorant receptors are novel seven transmembrane domain proteins that can signal independently of heterotrimeric $G$ proteins. Insect Biochem. Mol. Biol. 38, 770-780.

Smith, J.J., Kuraku, S., Holt, C., Sauka-Spengler, T., Jiang, N., Campbell, M.S., Yandell, M.D., Manousaki, T., Meyer, A., Bloom, O.E., et al. (2013). Sequencing of the sea lamprey (Petromyzon marinus) genome provides insights into vertebrate evolution. Nat. Genet. 45, 415-421e2. 
Sombke, A., Lipke, E., Kenning, M., Müller, C.H.G., Hansson, B.S., and Harzsch, S. (2012). Comparative analysis of deutocerebral neuropils in Chilopoda (Myriapoda): implications for the evolution of the arthropod olfactory system and support for the Mandibulata concept. BMC Neurosci. 13, 1-17.

Sorensen, P., and Caprio, J. (1998). Chemoreception. In The Physiology of Fishes, D. Evans, ed. (Boca Raton, FL: CRC), pp. 375-405.

Sorensen, P.W., Fine, J.M., Dvornikovs, V., Jeffrey, C.S., Shao, F., Wang, J., Vrieze, L.A., Anderson, K.R., and Hoye, T.R. (2005). Mixture of new sulfated steroids functions as a migratory pheromone in the sea Lamprey. Nat. Chem. Biol. 1, 324-328.

Speca, D.J., Lin, D.M., Sorensen, P.W., Isacoff, E.Y., Ngai, J., and Dittman, A.H. (1999). Functional identification of a goldfish odorant receptor. Neuron 23, 487-498.

Spehr, M., and Munger, S.D. (2009). Olfactory receptors: G protein-coupled receptors and beyond. J. Neurochem. 109, 1570-1583.

Stensmyr, M.C., Dweck, H.K.M., Farhan, A., Ibba, I., Strutz, A., Mukunda, L., Linz, J., Grabe, V., Steck, K., Lavista-Llanos, S., et al. (2012). A conserved dedicated olfactory circuit for detecting harmful microbes in drosophila. Cell 151, 1345-1357.

Stocker, R.F., Lienhard, M.C., Borst, A., and Fischbach, K.F. (1990). Neuronal architecture of the antennal lobe in Drosophila melanogaster. Cell Tissue Res. 262, 9-34.

Stowers, L., Holy, T.E., Meister, M., Dulac, C., and Koentges, G. (2002). Loss of sex discrimination and male-male aggression in mice deficient for TRP2. Science. 295, 1493-1500.

Strausfeld, N.J., and Hildebrand, J.G. (1999). Olfactory systems: Common design, uncommon origins? Curr. Opin. Neurobiol. 9, 634-639.

Suárez, R., Villalón, A., Künzle, H., and Mpodozis, J. (2009). Transposition and intermingling of Gai2 and Gao afferences into single vomeronasal glomeruli in the Madagascan lesser tenrec Echinops telfairi. PLoS One 4, e8005.

Suárez, R., Fernández-Aburto, P., Manger, P.R., and Mpodozis, J. (2011). Deterioration of the Gao vomeronasal pathway in sexually dimorphic mammals. PLoS One 6, e26436.

Sun, H., Kondo, R., Shima, A., Naruse, K., Hori, H., and Chigusa, S.I. (1999). Evolutionary analysis of putative olfactory receptor genes of medaka fish, Oryzias latipes. Gene 231, 137-145.

Syed, A.S., Sansone, A., Nadler, W., Manzini, I., and Korsching, S.I. (2013). Ancestral amphibian v2rs are expressed in the main olfactory epithelium. Proc. Natl. Acad. Sci. 110, 7714-7719.

Syed, A.S., Sansone, A., Hassenklöver, T., Manzini, I., Korsching, S.I. (2017). Coordinated shift of olfactory amino acid responses and $\mathrm{V} 2 \mathrm{R}$ expression to an amphibian water nose during metamorphosis. Cell. Mol. Life Sci. 74, 1711-1719.

Takami, S., and Graziadei, P.P.C. (1991). Light microscopic golgi study of mitral/tufted cells in the accessory olfactory bulb of the adult rat. J. Comp. Neurol. 311, 65-83.

Takigami, S. (2000). Projection Pattern of Vomeronasal Neurons to the Accessory Olfactory Bulb in Goats. Chem. Senses 25, 387-393.

Takigami, S., Mori, Y., Tanioka, Y., and Ichikawa, M. (2004). Morphological evidence for two types of mammalian vomeronasal system. Chem. Senses 29, 301-310. 
Taniguchi, K., Toshima, Y., Saito, T.R., and Taniguchi, K. (1996). Development of the olfactory epithelium and vomeronasal organ in the japanese reddish frog, Rana japonica. J. Vet. Med. Sci. 58, 715.

Taylor, G.M., Nol, E., and Boire, D. (1995). Brain regions and encephalization in anurans: adaptation or stability? Brain. Behav. Evol. 45, 96-109.

Tenne-Brown, J., and Key, B. (1999). Errors in lamina growth of primary olfactory axons in the rat and mouse olfactory bulb. J. Comp. Neurol. 410, 20-30.

Terni, B., Pacciolla, P., Masanas, H., Gorostiza, P., and Llobet, A. (2017). Tight temporal coupling between synaptic rewiring of olfactory glomeruli and the emergence of odor-guided behavior in Xenopus tadpoles. J. Comp. Neurol. 525, 3769- 3783.

Terni, B., Pacciolla, P., Perelló, M., and Llobet, A. (2018). Functional evaluation of olfactory pathways in living Xenopus tadpoles. J. Vis. Exp. 142, 10.3791/58028.

Terrapon, N., Li, C., Robertson, H.M., Ji, L., Meng, X., Booth, W., Chen, Z., Childers, C.P., Glastad, K.M., Gokhale, K., et al. (2014). Molecular traces of alternative social organization in a termite genome. Nat. Commun. 5, 3636.

Theisen, B. (1973). The olfactory system in the hagfish Myxine glutinosa I. fine structure of the apical part of the olfactory epithelium. Acta Zool. 54, 271-284.

Thewissen, J.G.M., and Nummela, S. (2008). Sensory evolution on the threshold: Adaptations in secondarily aquatic vertebrates (Oakland, CA: University of California Press).

Tomiyasu, J., Kondoh, D., Sakamoto, H., Matsumoto, N., Sasaki, M., Kitamura, N., Haneda, S., and Matsui, M. (2017). Morphological and histological features of the vomeronasal organ in the brown bear. J. Anat. 231, 749-757.

Toyoda, F., Hayakawa, Y., Kikuyama, S., and Ichikawa, M. (1999). Olfactory responses to a femaleattracting pheromone in the newt, Cynops pyrrhogaster. In Advances in Chemical Signals in Vertebrates, R. Johnston, D. Müller-Schwarze, and P.W. Sorensen, eds. (New York: Springer Science+Business Media), pp. 607-615.

Trahms, O.K. (1936). Das Geruchsorgan von Pipa americana. Z. Anat. Entwicklungsgesch. 105, 678693.

Troemel, E.R., Chou, J.H., Dwyer, N.D., Colbert, H.A., and Bargmann, C.I. (1995). Divergent seven transmembrane receptors are candidate chemosensory receptors in C. elegans. Cell 83, 207-218.

Tuchina, O., Koczan, S., Harzsch, S., Rybak, J., Wolff, G., Strausfeld, N.J., and Hansson, B.S. (2015). Central projections of antennular chemosensory and mechanosensory afferents in the brain of the terrestrial hermit crab (Coenobita clypeatus; Coenobitidae, Anomura). Front. Neuroanat. 9, 94.

Vassar, R., Ngai, J., and Axel, R. (1993). Spatial segregation of odorant receptor expression in the mammalian olfactory epithelium. Cell 74, 309-318.

Vassar, R., Chao, S.K., Sitcheran, R., Nuñez, J.M., Vosshall, L.B., and Axel, R. (1994). Topographic organization of sensory projections to the olfactory bulb. Cell 79, 981-991.

Veeranagoudar, D.K., Shanbhag, B.A., and Saidapur, S.K. (2004). Mechanism of food detection in the tadpoles of the bronze frog Rana temporalis. Acta Ethol. 7, 37-41. 
Venkatesh, B., Lee, A.P., Ravi, V., Maurya, A.K., Lian, M.M., Swann, J.B., Ohta, Y., Flajnik, M.F., Sutoh, Y., Kasahara, M., et al. (2014). Elephant shark genome provides unique insights into gnathostome evolution. Nature 505, 174-179.

Vieira, F.G., and Rozas, J. (2011). Comparative genomics of the odorant-binding and chemosensory protein gene families across the arthropoda: Origin and evolutionary history of the chemosensory system. Genome Biol. Evol. 3, 476-490.

Vielma, A., Ardiles, A., Delgado, L., and Schmachtenberg, O. (2008). The elusive crypt olfactory receptor neuron: Evidence for its stimulation by amino acids and cAMP pathway agonists. J. Exp. Biol. 211, 2417-2422.

Villamayor, P.R., Cifuentes, J.M., Fdz.-de-Troconiz, P., and Sanchez-Quinteiro, P. (2018). Morphological and immunohistochemical study of the rabbit vomeronasal organ. J. Anat.

Villinger, J., and Waldman, B. (2005). MHC-based kin recognition in African claewd frog tadpoles. News Zeal. J. Zool. 32, 230.

Vizueta, J., Frías-López, C., Macías-Hernández, N., Arnedo, M.A., Sánchez-Gracia, A., and Rozas, J. (2017). Evolution of chemosensory gene families in arthropods: Insight from the first inclusive comparative transcriptome analysis across spider appendages. Genome Biol. Evol. 9, 178-196.

Vogt, R.G., and Riddiford, L.M. (1981). Pheromone binding and inactivation by moth antennae. Nature 293, 161-163.

Vosshall, L.B., Amrein, H., Morozov, P.S., Rzhetsky, A., and Axel, R. (1999). A spatial map of olfactory receptor expression in the Drosophila antenna. Cell 96, 725-736.

De Vry, J., Martínez-Martìnez, P., Losen, M., Temel, Y., Steckler, T., Steinbusch, H.W.M., De Baets, M.H., and Prickaerts, J. (2010). In vivo electroporation of the central nervous system: A non-viral approach for targeted gene delivery. Prog. Neurobiol. 92, 227-244.

Vyas, R.N., Meredith, D., and Lane, R.P. (2017). Lysine-specific demethylase-1 (LSD1) depletion disrupts monogenic and monoallelic odorant receptor (OR) expression in an olfactory neuronal cell line. Mol. Cell. Neurosci. 82, 1-11.

Wabnitz, P.A., Bowie, J.H., Tyler, M.J., Wallace, J.C., and Smith, B.P. (1999). Aquatic sex pheromone from a male tree frog. Science. 401, 444-445.

Wachowiak, M., and Ache, B.W. (1994). Morphology and physiology of multiglomerular olfactory projection neurons in the spiny lobster. J. Comp. Physiol. A 175, 35-48.

Wagner, S., Gresser, A.L., Torello, A.T., and Dulac, C. (2006). A multireceptor genetic approach uncovers an ordered integration of VNO sensory inputs in the accessory olfactory bulb. Neuron 50 , 697-709.

Wakabayashi, Y., and Ichikawa, M. (2008). Localization of G protein alpha subunits and morphology of receptor neurons in olfactory and vomeronasal epithelia in Reeve's Turtle, Geoclemys reevesii. Zoolog. Sci. 25, 178-187.

Wakisaka, N., Miyasaka, N., Koide, T., Masuda, M., Hiraki-Kajiyama, T., and Yoshihara, Y. (2017). An adenosine receptor for olfaction in fish. Curr. Biol. 27, 1-11.

Waldman, B. (1991). Kin recognition in amphibians. In Kin Recognition, P. Hepper, ed. (Cambridge, UK: Cambridge University Press), pp. 162-219. 
Wang, R.T., and Halpern, M. (1980a). Scanning electron microscopic studies of the surface morphology of the vomeronasal epithelium and olfactory epithelium of garter snakes. Am. J. Anat. 157, 399-428.

Wang, R.T., and Halpern, M. (1980b). Light and electron microscopic observations on the normal structure of the vomeronasal organ of garter snakes. J. Morphol. 106, 56-68.

Ward, P., Labandeira, C., Laurin, M., and Berner, R.A. (2006). Confirmation of Romer's Gap as a low oxygen interval constraining the timing of initial arthropod and vertebrate terrestrialization. Proc. Natl. Acad. Sci. U. S. A. 103, 16818-16822.

Warren, W.C., Hillier, L.D.W., Marshall Graves, J.A., Birney, E., Ponting, C.P., Grützner, F., Belov, K., Miller, W., Clarke, L., Chinwalla, A.T., et al. (2008). Genome analysis of the platypus reveals unique signatures of evolution. Nature 453, 175-183.

Wassersug, R.J. (1980). Internal oral features of larvae from eight Anuran families: functional, systematic, evolutionary, and ecological considerations (Lawrence: University of Kansas).

Webre, D.J., Wolanin, P.M., and Stock, J.B. (2003). Bacterial chemotaxis. Curr. Biol. 13, 47-49.

Weiss, L., Offner, T., Hassenklöver, T., and Manzini, I. (2018). Dye electroporation and imaging of calcium signaling in Xenopus nervous system. In Methods in Molecular Biology, pp. 217-231.

Weiss, L., Jungblut, L.D., Pozzi, A.G., Zielinski, B.S., O’Connell, L.A., Hassenklöver, T., and Manzini, I. (2020). Multi-glomerular projection of single olfactory receptor neurons is conserved among amphibians. J. Comp. Neurol. 1-15.

Wells, K.D. (2007). The ecology and behavior of amphibians (Chicago: University of Chicago Press).

Wicher, D., Schäfer, R., Bauernfeind, R., Stensmyr, M.C., Heller, R., Heinemann, S.H., and Hansson, B.S. (2008). Drosophila odorant receptors are both ligand-gated and cyclic-nucleotide- activated cation channels. Nature 452, 1007-1011.

Van Wijk, M., Wadman, W.J., and Sabelis, M.W. (2006). Morphology of the olfactory system in the predatory mite Phytoseiulus persimilis. Exp. Appl. Acarol. 40, 217-229.

Wilburn, D.B., Doty, K.A., Chouinard, A.J., Eddy, S.L., Woodley, S.K., Houck, L.D., and Feldhoff, R.C. (2017). Olfactory effects of a hypervariable multicomponent pheromone in the redlegged salamander, Plethodon shermani. PLoS One 12, e0174370.

Wirsig-Wiechmann, C.R., Houck, L.D., Wood, J.M., Feldhoff, P.W., and Feldhoff, R.C. (2006). Male pheromone protein components activate female vomeronasal neurons in the salamander Plethodon shermani. BMC Neurosci. 7, 1-9.

Wittmer, C., and Nowack, C. (2017). Epithelial crypts: A complex and enigmatic olfactory organ in African and South American lungfish (Lepidosireniformes, Dipnoi). J. Morphol. 278, 791- 800.

Wong, S.T., Trinh, K., Hacker, B., Chan, G.C.K., Lowe, G., Gaggar, A., Xia, Z., Gold, G.H., and Storm, D.R. (2000). Disruption of the type III adenylyl cyclase gene leads to peripheral and behavioral anosmia in transgenic mice. Neuron 27, 487-497.

Woodley, S. (2015). Chemosignals, hormones, and amphibian reproduction. Horm. Behav. 68, 3-13.

Xu, Z., and Li, Q. (2020). TAAR Agonists. Cell. Mol. Neurobiol. 40, 257-272.

Yabuki, Y., Koide, T., Miyasaka, N., Wakisaka, N., Masuda, M., Ohkura, M., Nakai, J., Tsuge, K., Tsuchiya, S., Sugimoto, Y., et al. (2016). Olfactory receptor for prostaglandin F2 $\alpha$ mediates male fish courtship 
behavior. Nat. Neurosci. 19, 897-904.

Yamamoto, K., Kawai, Y., Hayashi, T., Ohe, Y., Hayashi, H., Toyoda, F., Kawahara, G., Iwata, T., and Kikuyama, S. (2000). Silefrin, a sodefrin-like pheromone in the abdominal gland of the sword-tailed newt, Cynops ensicauda. FEBS Lett. 472.

Yan, H., Jafari, S., Pask, G., Zhou, X., Reinberg, D., and Desplan, C. (2020). Evolution, developmental expression and function of odorant receptors in insects. J. Exp. Biol. 223, jeb208215.

Yang, C., and Delay, R.J. (2010). Calcium-activated chloride current amplifies the response to urine in mouse vomeronasal sensory neurons. J. Gen. Physiol. 135, 3-13.

Yang, L., Jiang, H., Wang, Y., Lei, Y., Chen, J., Sun, N., Lv, W., Wang, C., Near, T.J., and He, S. (2019). Expansion of vomeronasal receptor genes (OlfC) in the evolution of fright reaction in Ostariophysan fishes. Commun. Biol. 2, 235.

Yilmazer-Hanke, D.M., Hudson, R., and Distel, H. (2000). Morphology of developing olfactory axons in the olfactory bulb of the rabbit (Oryctolagus cuniculus): A Golgi study. J. Comp. Neurol. 426, 68-80.

Yoder, A.D., and Larsen, P.A. (2014). The molecular evolutionary dynamics of the vomeronasal receptor (class 1) genes in primates: A gene family on the verge of a functional breakdown. Front. Neuroanat. $8,153$.

Yohe, L.R., and Brand, P. (2018). Evolutionary ecology of chemosensation and its role in sensory drive. Curr. Zool. 64, 525-533.

Yohe, L.R., Abubakar, R., Giordano, C., Dumont, E., Sears, K.E., Rossiter, S.J., and Dávalos, L.M. (2017). Trpc2 pseudogenization dynamics in bats reveal ancestral vomeronasal signaling, then pervasive loss. Evolution (N. Y). 71, 923-935.

Yohe, L.R., Hoffmann, S., and Curtis, A. (2018). Vomeronasal and olfactory structures in bats revealed by dicect clarify genetic evidence of function. Front. Neuroanat. 12, 1-13.

Yokosuka, M., Hagiwara, A., Saito, T.R., Tsukahara, N., Aoyama, M., Wakabayashi, Y., Sugita, S., and Ichikawa, M. (2009a). Histological properties of the nasal cavity and olfactory bulb of the Japanese jungle crow corvus macrorhynchos. Chem. Senses 34, 581-593.

Yokosuka, M., Hagiwara, A., Saito, T.R., Aoyama, M., Ichikawa, M., and Sugita, S. (2009b). Morphological and Histochemical Study of the Nasal Cavity and Fused Olfactory Bulb of the BrownEared Bulbul, Hysipetes amaurotis . Zoolog. Sci. 26, 713-721.

Yonekura, J., and Yokoi, M. (2008). Conditional genetic labeling of mitral cells of the mouse accessory olfactory bulb to visualize the organization of their apical dendritic tufts. Mol. Cell. Neurosci. 37, 708718.

Yopak, K.E., Lisney, T.J., Darlington, R.B., Collin, S.P., Montgomery, J.C., and Finlay, B.L. (2010). A conserved pattern of brain scaling from sharks to primates. Proc. Natl. Acad. Sci. U. S. A. 107, 1294612951.

Yopak, K.E., Lisney, T.J., and Collin, S.P. (2015). Not all sharks are "swimming noses": variation in olfactory bulb size in cartilaginous fishes. Brain Struct. Funct. 220, 1127-1143.

Young, J.M., and Trask, B.J. (2007). V2R gene families degenerated in primates, dog and cow, but expanded in opossum. Trends Genet. 23, 212-215. 
Young, J.M., Massa, H.F., Hsu, L., and Trask, B.J. (2010). Extreme variability among mammalian V1R gene families. Genome Res. 20, 10-18.

Zacharuk, R.Y. (1980). Ultrastructure and function of insect chemosensilla. Annu. Rev. Entomol. 25, 2747.

Zapiec, B., and Mombaerts, P. (2020). The zonal organization of odorant receptor gene choice in the main olfactory epithelium of the mouse. Cell Rep. 30, 4220-4234.e5.

Zhang, X., and Firestein, S. (2002). The olfactory receptor gene superfamily of the mouse. Nat. Neurosci. 5, 124-133.

Zhao, Z., and McBride, C.S. (2020). Evolution of olfactory circuits in insects. J. Comp. Physiol. A 206, 353-367.

Zhao, H., Ivic, L., Otaki, J.M., Hashimoto, M., Mikoshiba, K., and Firestein, S. (1998). Functional expression of a mammalian odorant receptor. Science. 279, 237-242.

Zhu, G., Tang, W., Wang, L., Wang, C., and Wang, X. (2016). Identification of a uniquely expanded V1R ( ORA ) gene family in the Japanese grenadier anchovy ( Coilia nasus ). Mar. Biol. 163, 1-15.

\section{Acknowledgements}

There is a lot to be thankful for, if you have had the freedom to spend four years following pure curiosity and scientific drive to work on the olfactory system of amphibians. In the first place I would like to thank my family from the bottom of my heart for always letting me go my way and supporting me, however stubborn my decisions might have been sometimes. Ich schätze mich sehr glücklich euch in meinem Leben zu haben und danke euch für all die Freiheiten und die bedingungslose Unterstützung. Another big thank goes to my friends from home, in particular Monus, Markus, Gretul, Julus and Bastul. They have been socias srl throughout this time and have supported me in my deepest diva moments, when we swang from the chandelier, when we lived like tomorrow didn't exist, like it didn't exist. I thank my two wonderful gossip girls Sinem and Shama for being loving and caring friends, when it hasn't been my day, my week, my month or even my year. I thank Unicante for the music, the songs we were singing, thanks for all the joy they were bringing. In particular, I want to thank Manu and Lisa for all the queerness and happiness when I saw stormy weathers moving in, when they were about to begin. I thank Madlen and Lucas for looking at the stars with me, look how they shine for me. I thank Felix for being there when the color of the world was changing, day by day. I thank Julia, auch wenn die armen Dinos alle eingegangen sind. I thank Annika, because she called me up, when this world made me crazy and I've taken all I could bear. I thank Cille, Jakob, Manu and Damian for being there when I was in too deep and I have lost my mind, and I didn't care because they were there that night. I thank Andrés and Marcel for being there, cause that's much betta, betta, betta, betta. I thank the Neuros in Göttingen, particularly Rashad and Albert (insert Gladiator soundtrack here) and Kanishk, who was with me when the rain was blowing in my face, and the whole world was on my case. I would 
like to thank Leo, because a cat's the only pet who knows where it's at. A major thank goes to Sara and Tom, you were always like siblings to me, even though I don't have siblings and don't known how that feels, but in the end it doesn't even matter. I thank Melina, Daniela, Lukas, Kathi, Yvonne, Paola and our Bachelor and Master students for making the lab a better place, for you and for me, you and for me, you and for me. A big thank goes to Ivan for all his support over the years, his trust in my abilities and providing me with a scientific project that I could shape and grow with. I thank Thomas for his support and all the scientific tools and knowledge he provided me with and Anja for taking care of our animals and keeping the lab running. A big thanks to my TAC members Michael Hörner, Ralf Heinrich, Camin Dean and Martin Göpfert, who supported me with valuable feedback, motivation and advise. Thank you Sandra for the constant support and flexibility that made PhD life so much easier. I thank our collaborators Lucas Jungblut and Andrea Pozzi in Buenos Aires, Barbara Zielinski in Windsor and Lauren O'Connell at Stanford. Thanks for supporting my project and hosting me in your labs, I had a wonderful time both personally and scientifically. Finally, I want to thank Fran and Niles, RuPaul, Phoebe, Ross, Rachel, Monica, Chandler and Joey for letting me be part of their world and of course the Academy for the award. 


\section{List of Abbreviations}

(Abbreviations for Introduction and General Discussion)

\begin{tabular}{|c|c|}
\hline A & medial cluster \\
\hline AA & MC epithelium.....................................middle cavity \\
\hline ACIII................................. adenylate cyclase III & ..................main olfactory bulb \\
\hline AOB.......................... accessory olfactory bulb & .......main olfactory epithelium \\
\hline ATP .............................adenosine triphosphate & MS4 ..............membrane spanning four pass recep \\
\hline cAMP...... cyclic adenosine monophosphate & ..........olfactory \\
\hline beOE.................. buccal exposed epithelium & ..............olfactory epithel \\
\hline CNG........cyclic nucleotide gated ion channel & 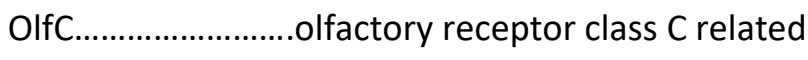 \\
\hline 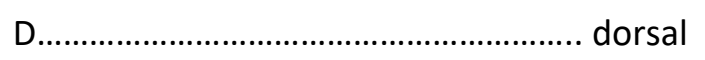 & ...................... type odorant rece \\
\hline DAG............................................. diacylglycerol & $\ldots \ldots \ldots \ldots . . .0$ olfactory receptor class A $\mathrm{r}$ \\
\hline DC ....................................... & Orco.. \\
\hline dMOB................... dorsal main olfactory bulb & ...........poste \\
\hline FPR ............................. formyl peptide receptor & ............................ principal cav \\
\hline ...receptor guanylyl Cyclase D & $\ldots \ldots \ldots \ldots \ldots \ldots \ldots \ldots \ldots$ olfactory receptor net \\
\hline GPCR....................G protein & ...........trace amine associated recep \\
\hline 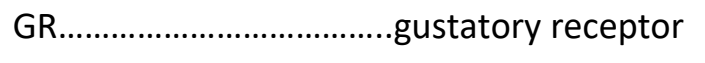 & TRPC2 ..........transient receptor potential channel \\
\hline IC........................................ntermediate cluster & .......ve \\
\hline IP3 ....................................inositol triphosphate & ............vomeronasal type 1 rece \\
\hline IR & V2R \\
\hline 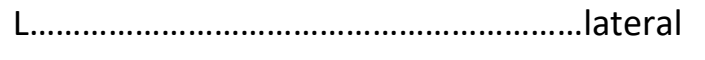 & ........ventral main olfactory $b$ \\
\hline 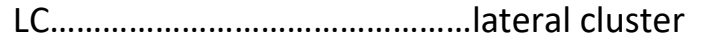 & .....vomeronasal o \\
\hline
\end{tabular}

\section{List of Figures}

(Figures for Introduction and General Discussion)

FIGURE 1 OLFACTORY PATHWAYS IN INVERTEBRATES.

FIGURE 2 OLFACTORY RECEPTORS IN VERTEBRATES.

FIGURE 3 TYPES OF VERTEBRATE RN AND THEIR INTRACELLULAR SIGNALING PATHWAYS. 13

FIGURE 4 SUBSYSTEM SEGREGATION IN VERTEBRATE OLFACTORY SYSTEMS.

FIGURE 5 GLOMERULAR WIRING IN THE VERTEBRATE OB.

FIGURE 6 PATHWAYS FOR AQUATIC AND AERIAL OLFACTION IN ANURANS. 100

FIGURE 7 LEFT-RIGHT INTEGRATION IN THE ANURAN AIR-BULB.

FIGURE 8 UNI- AND MULTI-GLOMERULAR WIRING IN OLFACTORY SYSTEMS. 108 


\section{Declaration}

Herewith I declare that I prepared the Doctoral Thesis "Information processing in the olfactory system of different amphibian species" on my own and with no other sources and aids than quoted.

Göttingen, 30.06.2020

Lukas Weiss 